
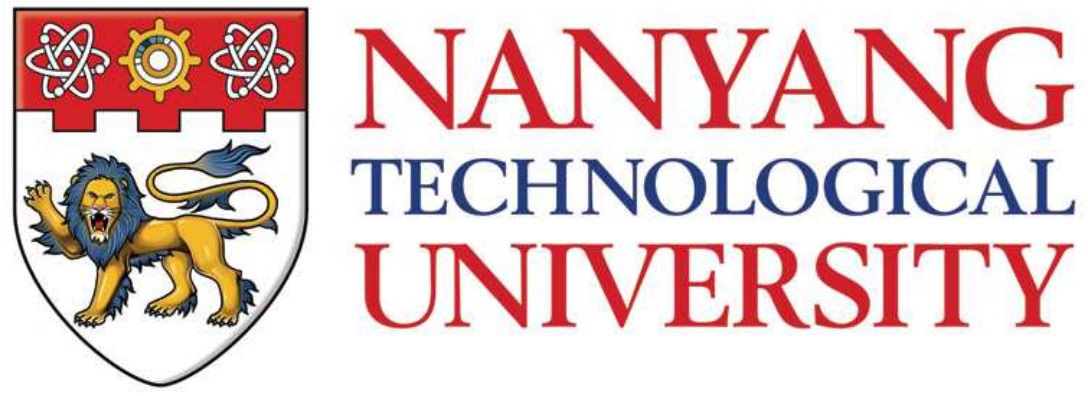

\title{
INTEGRATED CIRCUITS DESIGN AND CONTROL SOLUTIONS FOR WIRELESS POWER TRANSFER APPLICATIONS
}

\author{
by \\ BAI XIAOYIN
}

A Thesis

Submitted to the Faculty

of the

NANYANG TECHNOLOGICAL UNIVERSITY

in partial fulfillment of the requirements for the degree of

Doctor of Philosophy

in

Electrical and Electronic Engineering

2017 


\section{ACKNOWLEDGEMENTS}

As this $\mathrm{PhD}$ research journey comes to an end, it is time to express my sincere gratitude and to mention all of the great people who have constantly helped and encouraged me along the way. They have made over a thousand arduous days of study and work into bright memories. This dissertation belongs not only to the author but also to all these selfless people. First of all, I would like to express my deep respect for my supervisor, Assistant Professor Kong Zhi Hui. Although I was one of her first research students, she led me through the research process as though she had had decades of experience. I thank her for giving me the opportunity to work on this project and for her continuous support, guidance, and tolerance. I will never forget the constructive discussions we had during the past four years. Her encouragement made me feel brave when confronting the unpredictable challenges in my research life.

I would like to express my gratitude to Assoc. Professor Siek Liter, a man who has devoted his life to the field of integrated circuits. He is erudite, knowledgeable, friendly, and generous and has almost all the virtues a great professor could have. His enthusiasm and visionary understanding of the modern integrated circuit industry is what I would like to inherit in my future work. His office door was always open, and his precious and professional advice always kept me on track with the research direction.

I would like to thank Dr. Tan Yen Kheng for inspiring me with his work in the wireless power transfer field and for his contribution to my designs and drafts at critical times, offering wise, insightful, and comprehensive feedback. I would like to express my gratitude to Assoc. Professor Siek Liter, for his help 
and understanding of the modern integrated circuit industry. I would like to acknowledge Mr. Aaron H. Pereira, Mr. Robin Tan, and Mr. Yap Heng Goh for helping and teaching me to use various software tools and for helping me with the construction of the testing platform, with patience and proficient skills.

I would like to thank Dr. Sun Zhuo Chao, Dr. Zhu Di, Mr. Wang Jia Cheng, Mr. Zhang Xiang, and Mr. Yang Yong Kui for sharing with me their understanding of power electronics and analogue circuitry, which benefited me greatly. I would also like to thank Dr. Yao En Yi, Dr. Huang Nan, Miss. Chen Zihao, Mr. Feng Guang Ying, Mr. Xiao Zhe Kai, Miss Low Qiong Wwi, Mr. Aaron Cai, and all my other friends at VIRTUS for accompanying me during my $\mathrm{PhD}$ and for their myriad forms of support.

I would like to acknowledge financial support from Panasonic for the JIP Scholarship, as well as support from ERIAN.

There is someone who always shares my laughter and tears, someone who bears my complaints and tedium, someone who makes me loved without loneliness, someone who cheers me up when I feel hopeless. Thank you, Jiajia, from the bottom of my heart! Finally, I would like to thank my parents for all of their support from the day I came into this world. 


\begin{abstract}
In the past years, extensive research has been carried out on wireless power transfer (WPT), since it offers customers a convenient charging technique compared to wires which can be inconvenient and allows charging of portable devices in a wide power range. WPT technology has been observed to have some remarkable technological advantages due to its spatial freedom. Owing to modern power electronics technology and high-power semiconductors, WPT technology is highly integrated, and many smart WPT devices have been developed for different applications such as biomedical devices, electric vehicles (EV), and portable consumer electronic devices. A burst in the WPT market for consumer electronics is expected in the coming five years, due to the ubiquitous usage of $3 \mathrm{G} / 4 \mathrm{G}$ wireless networks and continually evolving technologies for media and entertainment. WPT systems for consumer devices can be classified into two main types: inductive power transfer (IPT) and magnetic resonant coupling (MRC). The former works at $100-200 \mathrm{kHz}$, whereas the latter works at 6.78 or $13.56 \mathrm{MHz}$. The bottleneck of the IPT technology is to design a low cost but high performance WPT system since the IPT mainly targets on the application with less requirement of distance freedom like home appliance or some industrial applications with short charging distance. The main targets of MRC technology is to provide consumers a flexible WPT solution with higher distance freedom. The main concern of this technology is that 1) it is challenging to design high efficiency circuits for MHz application, especially for rectifier; 2) the control of the MRC is challenging since the output power would be effected remarkably by distance, which is known as splitting frequency phenomenon.
\end{abstract}


This research develops circuits and control algorithms for both types of WPT systems. Firstly, a single-stage AC-DC voltage regulator is developed. A noncharging configuration is introduced to regulate the output voltage at a desired level with a pulse width modulation (PWM) control algorithm. In this way, there is no need for the DC-DC stage after the rectifier and additional wireless communication circuit blocks. In addition, a full-active cross-coupled structure is utilized to reduce the power loss of the regulator. Experimental results of the proposed regulator based on a $0.35 \mu \mathrm{m}$ technology and PCB circuit demonstrate a maximum output power of $15 \mathrm{~W}$ and peak efficiency of $92 \%$.

Another contribution of this dissertation is a design of a full active rectifier working at $6.78 \mathrm{MHz}$. An adaptive time delay (ATD) circuit is used to maximize the conduction interval of the gallium nitride $(\mathrm{GaN})$ switch in order to minimize the power loss due to the forward voltage drop of the diode. The proposed control algorithm also eliminates the reverse leakage current of the rectifier. Except the power devices, all the other circuits have been taped out with a $0.18 \mu \mathrm{m}$ CMOS process. The experimental results of the proposed rectifier shows that it can output a maximum output current of $3 \mathrm{~A}$ at $5 \mathrm{~V}$ with a $6.78-\mathrm{MHz} \mathrm{AC}$ input voltage with a peak power efficiency over $90 \%$.

The last contribution is a novel control method for a $6.78-\mathrm{MHz}$ MRC system. In this part, detailed analysis of MRC, especially on the relationship between operating frequency, transfer efficiency, output power, and coupling coefficient are presented. Contrary to the traditional research, this research proves that the MRC system is able to work with sufficient output power and efficiency within a wide coupling coefficient range, relaxing the system from the bound of the key coupling coefficient limitation. A hybrid control method with frequency/phaseshift tuning with zero voltage switching (ZVS) Class-D amplifier is proposed to provide the load with a constant output voltage. The testing results shows that it is able to transfer $10 \mathrm{~W}$ over a $5 \mathrm{~cm}$ with an overall efficiency (end to end) of $71.8 \%$, and over a $1 \mathrm{~cm}$ with an overall efficiency of $73.1 \%$. 


\section{TABLE OF CONTENTS}

ACKNOWLEDGEMENTS ................. i i

ABSTRACT ...........................

LIST OF FIGURES . . . . . . . . . . . . . . . . . . . viii

LIST OF TABLES . . . . . . . . . . . . . . . . . . xiv

LIST OF ABBREVIATIONS . . . . . . . . . . . . . . XV

\section{CHAPTER}

1. Introduction . . . . . . . . . . . . . . . . . 1

1.1 Background . . . . . . . . . . . . . 1

1.2 Motivation . . . . . . . . . . . . . 4

1.3 Objectives and Research Scope . . . . . . . . . 5

1.4 Thesis Contributions . . . . . . . . . . . . 5

1.5 Organization of the Thesis . . . . . . . . 7

2. Literature Review . . . . . . . . . . . . . . . . . . . 9

2.1 Basic concepts and principle of WPT . . . . . . . . 9

2.1.1 Magnetic coupling . . . . . . . . . . . . . 9 9

2.1.2 Coupled-Mode Theory . . . . . . . . . . . . 10

2.1.3 Maximum Power Transfer Principle and Maximum Transfer Efficiency Principle . . . . . 11

2.1.4 Topologies of WPT . . . . . . . . . . . 12

2.2 Control Circuits and Methods for WPT . . . . . . 20

2.2.1 Control at the transmitter side . . . . . . 20

2.2.2 Control at the receiver side . . . . . . . 22

2.3 High efficiency rectifier design . . . . . . . . . . 32

2.4 Frequency Splitting . . . . . . . . . . . . . . . . . . 38

2.5 Power Amplifier . . . . . . . . . . . . . . . . 40

2.5.1 Class D Amplifier . . . . . . . . . . . 40

2.5.2 Class E Amplifier . . . . . . . . . . . . . 42

2.6 Summary . . . . . . . . . . . . . . . . 46 46 
3.1 Introduction . . . . . . . . . . . . 47

3.2 Feasibility and Performance Analysis of the Novel Regulator . . . . . . . . . . . . . . . . 49

3.2.1 The Design of Non-Charging Configuration . 50

3.2.2 High Efficiency Operation Area Analysis . . 52

3.2.3 Safe Operation Area Analysis . . . . . . . 55

3.3 Proposed Single-Stage Voltage Regulator for Wireless Power Transfer . . . . . . . . . . . . . . 56

3.3.1 Structure and Operation of the Proposed SingleStage Voltage Regulator . . . . . . . . 56

3.3.2 Equivalent Model WPT System with SingleStage Voltage Regulator . . . . . . . . . 57

3.4 Circuit Structure and Key Blocks . . . . . . . . . . . 60

3.4.1 Main Comparator Design . . . . . . . . . . . 62

3.4.2 Ramp Generator . . . . . . . . . . . . . . 64

3.4.3 Start-up Circuit . . . . . . . . . . . . . . . 65

3.4.4 Digital Controller . . . . . . . . . . . . . . 66

3.5 Experimental Results . . . . . . . . . . . . . . . . . 67

3.6 Conclusions $\ldots \ldots \ldots \ldots \ldots \ldots \ldots \ldots$

4. Design of A Full Active Rectifier with Adaptive Time Delay Control for 6.78 MHZ Wireless Power Transmission . . . . 73

4.1 Introduction . . . . . . . . . . . . . . 73

4.2 Proposed Rectifier with Adaptive Time Delay (ATD) Control . . . . . . . . . . . . . . . . 75

4.2.1 Structure and Operating principles of the Proposed Rectifier . . . . . . . . . . . 75

4.2.2 Adaptive Time Delay Control Algorithm . . 77

4.2.3 Start-up Mechanism . . . . . . . . . . . . 80

4.3 Circuit Structure and Key Blocks . . . . . . . . . . . 82

4.3.1 Topology of the Proposed Rectifier . . . . . 82

4.3.2 Rising Edge Detector and Falling Edge Detector 83

4.3.3 Proximity Detector . . . . . . . . . . . . . 86

4.3.4 Digital Blocks . . . . . . . . . . . . . . 88

4.4 Experimental Results $\ldots \ldots \ldots \ldots$

4.5 Conclusions $\ldots \ldots \ldots \ldots \ldots$

5. Analysis and Hybrid Control Method for 6.78 MHZ Wireless Power Transfer System with Varying Transmission Distance

5.1 Introduction . . . . . . . . . . . . . 95

5.2 Analysis of Magnetic Resonant Wireless Power Transfer System . . . . . . . . . . . . . . . . . . . . . 96

5.2.1 System Overview . . . . . . . . . . . . . . . 96 
5.2.2 Maximum Efficiency of a Series-Series (S-S)

Compensation ............ . . 99

5.2.3 Frequency Splitting Analysis . . . . . . . . . 101

5.3 Voltage Control Method and Efficiency Optimization . 106

5.3.1 Voltage Control Method Description . . . . . 106

5.3.2 Parameter Design for Power Transfer Efficiency 108

5.3.3 Design of a Zero Voltage Switching (ZVS)

CLASS-D Amplifier . . . . . . . . . . 111

5.4 Experimental Results . . . . . . . . . . . . . . . 114

5.5 Conclusions . . . . . . . . . . . . . 118

6. Conclusions and Future Work . . . . . . . . . . . . . . 121

6.1 Conclusions . . . . . . . . . . . . 121

6.2 Future Work . . . . . . . . . . . . . . 123

APPENDICES . . . . . . . . . . . . . . . . . . 125

A. Phasor Transformation . . . . . . . . . . . . . . . . 127

B. Author's Publications _. . . . . . . . . . . . . . . 131

B.1 Journal Papers . . . . . . . . . . . . . . . . 131

B.2 Patent Proposals . . . . . . . . . . . . . . . 131

B.3 Conference Papers . . . . . . . . . . . . . . 132

BIBLIOGRAPHY . . . . . . . . . . . . . . . . . . 133 


\section{LIST OF FIGURES}

\section{Figure}

$1.1 \quad$ Nikola Tesla's experiment known as Wardenclyffe Tower in 19012

1.2 WPT technologies for three applications: (a) the EM wave WPT for unmanned plane receives power beamed from earth 21, (b) IPT WPT for a mobile phone 22 and (c) MRC WPT system developed by Intel using flat coils 23. . . . . . . . . . . . 2

2.1 (a) Equivalent circuit of the power transfer system, (b) variations of output power and transfer efficiency as a function of

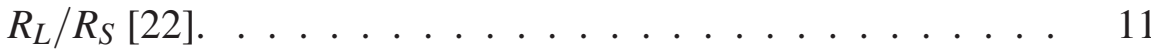

2.2 Topologies of four basic compensation network. . . . . . . . 13

2.3 The LCL and LCCL compensation topologies. . . . . . . . . 15

2.4 The experimental results of $[23] \ldots \ldots \ldots$

2.5 The equivalent circuit for SS model. . . . . . . . . . . . . 17

$2.6 \quad$ Schematic of four-coil WPT system. [31] . . . . . . . 18

2.7 Equivalent circuit model of four-coil WPT system. [14] . . . . 19

2.8 Qi wireless power transfer system [34]. . . . . . . . . . . . 21

2.9 Two different kinds of communication: (a) resistive (b) capaci-

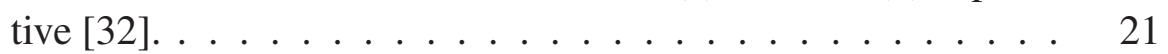

2.10 PID control algorithm [32]. . . . . . . . . . . . . 21

2.11 A4WP wireless power transfer system [33]. . . . . . . . 22

2.12 Wireless power transfer system in [12] . . . . . . . . 23

2.13 Basic DC-DC Topologies. . . . . . . . . . . . . 24 
2.14 Voltage mode PWM buck DC-DC converter [35]. . . . . . . 25

2.15 Current mode PWM buck DC-DC converter:(a) peak current control and (b) valley current mode control [35]. . . . . . . 26

2.16 PFM control buck converter with fixed on-time control [35]. . 27

2.17 PSM control buck converter in [38]. . . . . . . . . . 27

2.18 Hysteretic control DC-DC converter in [39]. . . . . . . . 27

2.19 An average-current mode DC-DC with PSM for a $6 \mathrm{~W}$ WPT in $[40] \ldots \ldots \ldots \ldots \ldots$

2.20 A hysteretic mode DC-DC with PLL for a 6 W WPT in [41]. . 29

2.21 The schematic of active reconfigurable voltage rectifier/doubler rectifier in [42]. . . . . . . . . . . . . . . . 30

2.22 The schematic of the $3 \mathrm{R}$ rectifier in [43]. . . . . . . . 31

2.23 The circuit model of the $1 \mathrm{X} / 2 \mathrm{X}$ reconfigurable rectifier in [44]. 31

2.24 The circuit model of the tri-state boost converter in [45]. . . . 32

2.25 The explanation of reverse current. . . . . . . . . . . 33

2.26 The circuit model of [46] . . . . . . . . . . . . . 33

2.27 The schematic of active rectifier with speed-up latch in [47]. . 34

2.28 The schematic of the rectifier with unbalanced biasing design

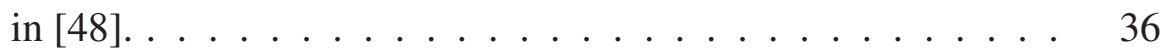

2.29 The schematic of a high-efficiency active rectifier with DLL in $[41] \ldots \ldots \ldots \ldots$. . . . . . . . . . . . . . . . . . . . . . . . .

2.30 The experiment results of critical coupling of 5-10 MHz WPT in [25](a) Comparison of (black dots) experimental data to the calculation results of the system; (b)Measured and Predicted critical coupling. . . . . . . . . . . . . . . 39

2.31 A typical Class D half-bridge amplifier:(a) Schematic.(b)Equivalent circuits. ................... 41

2.32 A Class D amplifier with power control in [54]. . . . . . . . 41

2.33 The analytical model in [55]. . . . . . . . . . . . 42 
2.34 Circuits and Waveforms in [56]. . . . . . . . . . 43

2.35 A typical Class E amplifier:(a) Schematic.(b)Equivalent circuits. 43

2.36 Analysis of Class E amplifier at different loads and frequencie in $[57] \ldots \ldots \ldots \ldots$. . . . . . . . . . . . . . . . . . 4 44

2.37 Equivalent circuits model Class E- Class E WPT system in [58]. 45

2.38 Design curves with the parameter $Q_{1}$ of [59]. . . . . . . 45

3.1 Wireless power transfer (WPT) system for consumer devices. . 47

3.2 Proposed WPT system with proposed single-stage regulator. . 49

3.3 Operation of a traditional buck DC-DC Topologies. . . . . . . 50

3.4 Two different non-configurations in WPT system (a) Disconnection mode; (b) Freewheeling loop mode. . . . . . . . . 51

$3.5 \quad$ Voltage Gain vs. M and Power Loss vs. M. . . . . . . . . 54

$3.6 \quad$ (a) $I_{1}$ and $V_{C 1}$ vs. M (b) $I_{2}$ and $V_{C 2}$ vs. M. . . . . . . . 55

3.7 Operation principle of the proposed regulator (a) overall structure (b) charging configuration (c) non-charging configuration (d) current of the receiving coil. . . . . . . . . . . 58

3.8 Model of the WPT system with the proposed single-stage voltage regulator. . . . . . . . . . . . . 5 59

3.9 Simulation result of $V_{R E G}$ vs. D. . . . . . . . . . 60

3.10 Circuit implementation of the proposed voltage regulator (a) Complete blocks of the regulator ; (b) Circuits for charging configuration; (c) Circuits for non-charging configuration. . . . . 61

3.11 Schematic of Main comparator $\left(C M P_{2}\right) \ldots \ldots 63$

3.12 The leakage of the MOSFET . . . . . . . . . . . 64

3.13 Schematic of Ramp generator. . . . . . . . . . . 65

3.14 Simulation results of the ramp generator. . . . . . . . 65

3.15 Schematic of Startup Circuit. . . . . . . . . . . . . 66 
3.16 Schematic of Digital Controller. . . . . . . . . . . 67

3.17 (a)Die photo and whole regulator on PCB.(b) Test bench . . . 68

3.18 Oscilloscope waveform: (a) Measured waveform with a load transient of between $15 \mathrm{~W}$ and $2 \mathrm{~W}$ at $4 \mathrm{~mm}$; (b) Measured waveform with a load transient of between $2 \mathrm{~W}$ and $15 \mathrm{~W}$ at 4 $\mathrm{mm}$; (c) Measured waveform with a load of $15 \mathrm{~W}$ at $8 \mathrm{~mm}$. . .

3.19 Measured efficiency of the regulator. . . . . . . . . . . .

3.20 (a) Load regulation from $25(1 \mathrm{~W})$ to $1.6(15 \mathrm{~W})$ at $20 \mathrm{~V}$ input and $4 \mathrm{~mm}$ distance; (b) Line regulation from $18 \mathrm{~V}$ to $24 \mathrm{~V}$ at 15 $\mathrm{W}$ at $4 \mathrm{~mm}$; (c) Distance regulation from $4 \mathrm{~mm}$ to $8 \mathrm{~mm}$ at $20 \mathrm{~V}$ input and $15 \mathrm{~W} . \ldots \ldots \ldots$. . . . . . . . . 70

4.1 Wireless power transfer (WPT) system. . . . . . . . 73

4.2 Topology and operation of the proposed active rectifier. . . . 76

4.3 Details of the adaptive time delay control. . . . . . . . . . 79

4.4 Timing of the adaptive time delay control. . . . . . . . . . 80

4.5 Timing diagram of the rectifier at start-up. . . . . . . . . 81

4.6 Topology of the proposed rectifier with detailed blocks. . . . . 82

4.7 Topology of the rising and falling edge detector. . . . . . . . . 84

4.8 Simulation results of the rising edge detector. . . . . . . 85

4.9 Simulation results of the falling edge detector. . . . . . . . 85

4.10 Scheme of the gate proximity detector. . . . . . . . . . 86

4.11 Simulation results of the proximity detector. . . . . . . . 87

4.12 Scheme of the TDC and the operation principle. . . . . . 88

4.13 Timing diagram of the digital control. . . . . . . . . . . 89

4.14 Schematic of the delay circuits. . . . . . . . . . . . 90

4.15 Die photo and whole rectifier with GaN FETs on PCB. . . . . 90 
4.16 (a) Waveform of the active rectifier at 5V/3A. (b) Zoom-in view of (a).(c) Waveform of the active rectifier at $5 \mathrm{~V} / 0.15 \mathrm{~A}$. (d) Zoom-in view of $(\mathrm{c}) . \ldots \ldots . \ldots . \ldots 92$

4.17 Measured efficiency of the rectifier. . . . . . . . . . . 93

$5.1 \quad$ Wireless power transfer (WPT) system. . . . . . . . 96

5.2 Simplified WPT circuit model: (a) Topology, (b) RLT model, (c) S-S circuit topology. . . . . . . . . . . . . . . . . 97

5.3 Efficiency with load variation. . . . . . . . . . . 100

$5.4 \quad$ Frequency splitting effect (Calculstion results). . . . . . . . 104

5.5 Frequency splitting effect (Calculstion and experimental results). 105

$5.6 \quad$ System control flow. . . . . . . . . . . . . . . . . 107

5.7 Frequency splitting effect (Efficiency drop at $\omega_{1}$ and $\omega_{2}$ : calculation and testing results.). . . . . . . . . . . 109

$5.8 \quad$ Efficiency at $\omega_{1}$ for different coupling value. . . . . . . . . 110

5.9 ZVS Class-D Amplifier. . . . . . . . . . . . . . . . 112

5.10 Operation of the ZVS. . . . . . . . . . . . 113

5.11 Voltage-Current waveform of the ZVS class-D amplifier. . . . 114

5.12 Test bench for the proposed 6.78-MHz WPT system. . . . . . 115

5.13 Oscilloscope waveform of the output voltage cross the load $\left(R_{L}=10 \Omega\right)$ and the power amplifier output voltage at $5 \mathrm{~cm}$. . 116

5.14 Oscilloscope waveform of the output voltage cross the load $\left(R_{L}=50 \Omega\right)$ and the power amplifier output voltage at $5 \mathrm{~cm}$. . 116

5.15 Oscilloscope waveform of the output voltage cross the load $\left(R_{L}=10 \Omega\right)$ and the power amplifier output voltage at $1 \mathrm{~cm}$. . .

5.16 Oscilloscope waveform of the output voltage cross the load $\left(R_{L}=50 \Omega\right)$ and the power amplifier output voltage at $1 \mathrm{~cm}$. . .

A.1 Inductor phasor transformation. (a) AC-Rotationary circuit. (b) DC-Stationary circuit. . . . . . . . . . . . 128 
A.2 Capacitor phasor transformation. (a) AC-Rotationary circuit. (b) DC-Stationary circuit. . . . . . . . . . . . . . . 129

A.3 Resistor phasor transformation. (a) AC-Rotationary circuit. (b) DC-Stationary circuit. . . . . . . . . . . . 130 


\section{LIST OF TABLES}

\section{Table}

1.1 Summary of three main wireless power transfer technology . . 3

$2.1 \quad$ A summary about the rectifier from [49] [48] [41] . . . . . 38

3.1 Design equations of Non-Connection and Free wheeling Mode 52

3.2 Mutual inductance from $4 \mathrm{~mm}$ to $8 \mathrm{~mm} \ldots \ldots 54$

$3.3 \quad$ Coil specifications . . . . . . . . . . . . . 68

3.4 Performance comparison with the proposed regulator . . . . 71

4.1 Testing components of the proposed 6.78-MHz Rectifier _. . 91

4.2 Performance comparison with the proposed rectifier . . . . . . 94

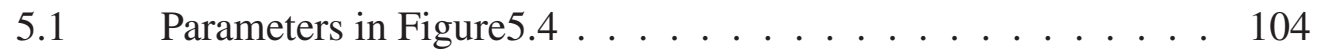

5.2 Testing components of the proposed 6.78-MHz WPT system . 115

5.3 Testing results of the proposed WPT system . . . . . . . . 118

5.4 Performance comparison with the proposed WPT system $\ldots 118$ 


\section{LIST OF ABBREVIATIONS}

\begin{tabular}{ll} 
WPT & wireless power transfer \\
IPT & inductive power transfer \\
MRC & magnetic resonant coupling \\
AC & alternating current \\
DC & direct current \\
EV & electric vehicles \\
CMT & coupled mode theory \\
ZPA & zero phase angle \\
M & mutual inductance \\
$L_{1}$ & self-inductance of the transmitting coil \\
$L_{2}$ & self-inductance of the receiving coil \\
ZVS & zero voltage switching \\
PCE & power conversion efficiency \\
DC & direct current \\
PWM & pulse width modulation \\
PFM & pulse frequency modulation \\
EMI & electro magnetic interference \\
PSM & pulse skipping modulation \\
CCM & continuous conduction mode \\
DCM & discontinuous conduction mode \\
PVT & process, voltage and temperature \\
THD & total harmonic distortion \\
\hline
\end{tabular}




$\begin{array}{ll}\text { GaN } & \text { gallium nitride } \\ \text { CMOS } & \text { complementary metal-oxide semiconductor } \\ \text { PCB } & \text { printed circuit board } \\ \text { RFID } & \text { radio-frequency identification } \\ \text { LCL } & \text { inductor-capacitor-inductor } \\ \text { LCCL } & \text { inductor-capacitor-capacitor-inductor } \\ \text { MOSFET } & \text { metal-oxide-semiconductor field-effect transistor } \\ \text { FPGA } & \text { field-programmable gate array } \\ \text { PID } & \text { proportional-integral-derivative } \\ \text { ATD } & \text { adaptive time delay } \\ \text { SS } & \text { series-series } \\ \text { SP } & \text { series-Parallel } \\ \text { PS } & \text { parallel-series } \\ \text { PP } & \text { parallel-parallel } \\ \text { VA } & \text { voltage-ampere } \\ \text { EM } & \text { electromagnetic }\end{array}$




\section{Chapter 1}

\section{Introduction}

\subsection{Background}

The idea of wireless power transfer can be dated back to 1901, when Nikola Tesla first attempted to carry out his famous Wardenclyffe Tower experiment (Figure 1.1) near Long Island, for transmitting power wirelessly [1]. Unfortunately, due to the limited radio-wave technology available in the early 1900s, his experiment to prove the feasibility of WPT was not successful. With the development of electromagnetic (EM) wave technology, WPT via EM has drawn researchers attention since the late 1970s [2] [3] [4] [5] [6].In an EM WPT system, a transmitter emits EM waves in a specific industrial, scientific, and medical (ISM) band (850-950 or 902-928 MHz) [6] and a receiver with the same frequency band catches and stores the EM power (Figure 1.2(a)). However, the trade-off between efficiency and transmission directionality hinders the development of this far-field approach [3] , [7].

The near-field IPT [8] [9] [10] [11] [12] [13] is becoming popular as a result of the fast development of portable consumer devices. The operation principle of an IPT system is EM induction, which is similar to that of a transformer. It is an efficient WPT system for short-distance applications because it is non-radiative so the energy is not wasted in the medium. IPT technology is widely used for mid-power home appliances such as mobile phones (Figure 1.2(b)) or electric 


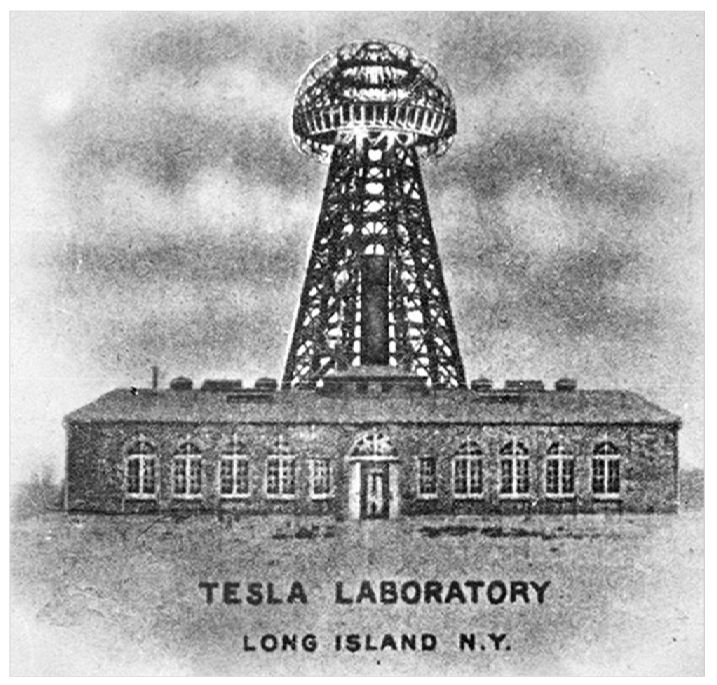

Figure 1.1: Nikola Tesla's experiment known as Wardenclyffe Tower in 1901

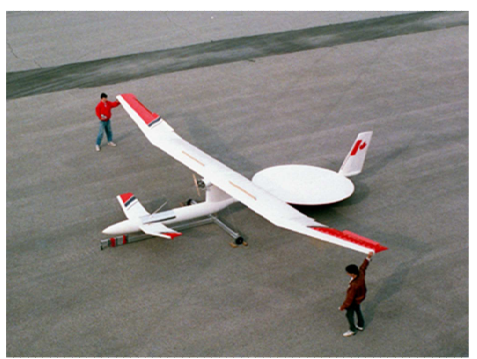

(a)

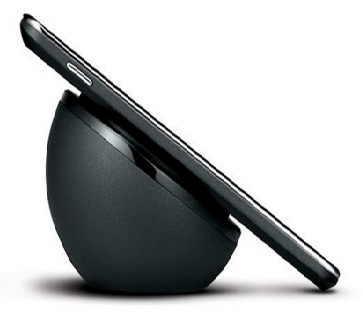

(b)

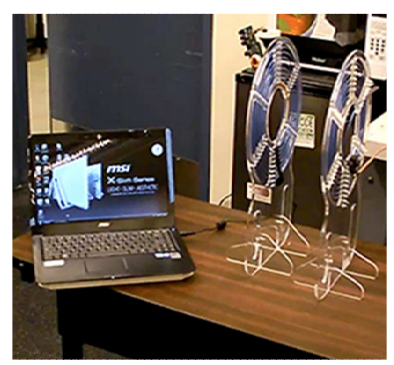

(c)

Figure 1.2: WPT technologies for three applications: (a) the EM wave WPT for unmanned plane receives power beamed from earth 21, (b) IPT WPT for a mobile phone 22 and (c) MRC WPT system developed by Intel using flat coils 23

toothbrushes, and efficiency of over $70 \%$ is possible for these applications. For high-power applications of up to several kilowatts, the efficiency of IPT technology could be as high as $90 \%$. The operating frequency range of IPT is generally from $20 \mathrm{KHz}$ to $1 \mathrm{MHz}$.

Despite the advantages of IPT technology, one problem is that its efficiency decreases as the distance or the position mismatch between the two coils becomes larger. A new technology, known as MRC WPT, has been developed to solve this problem. In 2007, an research group from Massachusetts Institute of Technology first conducted experiments using this technology to transfer $60 \mathrm{~W}$ over $100 \mathrm{~cm}$ with an efficiency of over $90 \%$ [14] [15]. In this experiment, two resonant coils with a Q-factor nearly as high as 1000 form a powerful resonator 
and thus enhance the efficiency of WPT over longer distances. Compared to EM WPT, resonant WPT is less sensitive to the environment [16]. Numerous research works have been carried out to accelerate the development of resonant WPT. Figure 1.2(c) shows a new resonant coupled WPT developed by Intel in 2008. In this system, a pair of planar coils are used, as they can be implanted into consumer devices more easily than helix coils [15]. Several single-transmitter to multi-receiver systems based on resonant WPT technology have been developed in recent years [17]. The operating frequency of this technology is from $1 \mathrm{MHz}$ to nearly $200 \mathrm{MHz}$ [18]. A summary of these three kinds of WPT technologies is given in Table 1.1

Table 1.1: Summary of three main wireless power transfer technology

\begin{tabular}{|c|c|c|c|}
\hline Types & Advantages & Disadvantages & Applications \\
\hline $\begin{array}{l}\text { EM } \\
\text { wave }\end{array}$ & $\begin{array}{l}\text { The size of receiver } \\
\text { is tiny } \\
\text { The transfer efficiency } \\
\text { is high over a wide } \\
\text { power and } \\
\text { load variations. }\end{array}$ & $\begin{array}{l}\text { Sensitive to distance } \\
\text { Only for low-power } \\
\text { applications }\end{array}$ & $\begin{array}{l}\text { Wireless sensor } \\
\text { network } \\
\text { Unmanned plane }\end{array}$ \\
\hline IPT & $\begin{array}{l}\text { High safety } \\
\text { High efficiency in short } \\
\text { transfer distance } \\
\text { High power transfer } \\
\text { capability in short } \\
\text { transfer distance }\end{array}$ & $\begin{array}{l}\text { Short transfer distance } \\
\text { Sensitive to alignment }\end{array}$ & $\begin{array}{l}\text { Portable electronic } \\
\text { devices such as cell } \\
\text { phones and laptops }\end{array}$ \\
\hline $\begin{array}{l}\text { MRC } \\
\text { WPT }\end{array}$ & $\begin{array}{l}\text { High efficiency } \\
\text { for mid-range } \\
\text { power transfer } \\
\text { Insensitive to } \\
\text { the environment }\end{array}$ & $\begin{array}{l}\text { Sensitive to alignment } \\
\text { Large size of coil }\end{array}$ & 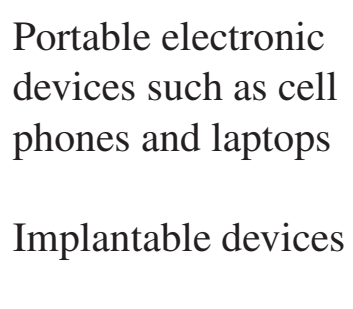 \\
\hline
\end{tabular}




\subsection{Motivation}

The progress of technology through the history of human kind has continually changed people's way of life. At the end of the 20th century, the development of personal computers brought us into the information age. At the beginning of the 21 st century, with the rapid growth of wireless communication, we are entering a new era of wireless technology. However, people still use wires to charge their wireless devices, which ironically cause wireless devices to become nonwireless. Wireless Power Transfer (WPT) is a suitable solution for this problem. With continuous efforts in improving the process of modern semiconductor industry, it seems more attractive than ever to develop a high performance WPT system with smart control and affordable cost.

Moreover, due to the widespread usage of technologies for entertainment such as high-definition video and virtual reality games, the escalating power consumption of electronic devices has become a growing concern in both the industrial and academic fields because the duration of device operation is quite limited . One solution is to increase the storage capacity of the battery, but this is limited to chemistry and materials science due to their current state of development. In that case, a flexible power transfers solution, like WPT, is considered to be appealing for consumers for the following reasons: 1) this technology expand the spatial freedom of charger and devices for users; 2) it is an effective power transfer method for unreachable loads, like several applications in the bio-medical application and automation industry; 3) without exposed conductors, it can be considered a safer power transfer process. Despite these advantages, it yet remains as a challenge task to design a high-performance WPT. It have a larger power loss compared to traditional plug-in chargers due to the power consumed in the extra circuits (like power amplifier, rectifiers, voltage regulator) and transmission coils. Besides, a WPT system consists of two separate parts without any physical contact so the power flow control becomes difficult. These advantages 
and challenges motivate the work in this thesis.

\subsection{Objectives and Research Scope}

The objectives of this research project are listed as follows:

- To improve the design and performance of circuits in WPT system targeting IPT WPT and MRC WPT by utilizing different circuit topologies;

- To develop smart, low cost, high performance output voltage control methods for IPT wireless power transfer system @ kHz;

- To develop a strategy for MRC technology @ MHz targeting at high-end WPT application to maintain the performance of the entire system within a large dynamic range of different parameters of the system, such as transferring distance, load conditions and so on;

In this research, WPT system and circuits are designed for consumer electronic devices. The power of the system and circuits is limited to $15 \mathrm{~W}$ and the output voltage is limited to below $10 \mathrm{~V}$. However, the applications of the system structure, the circuit structure, and the control algorithm are not limited to this power range if the benefits proven to be attractive for high-power applications and if the process can handle the required voltage and current. EM WPT systems with operating frequencies above $100 \mathrm{MHz}$ are not included in this thesis due to their low efficiency, low output power and safety issues.

\subsection{Thesis Contributions}

The main contributions of this thesis are as follows:

- Design of a single-stage 125-kHz IPT wireless power transfer receiver with a Pulse Width Modulation (PWM)-controlled active power stage. The output voltage is controlled at a constant value, such as $5 \mathrm{~V}$ in a mobile 
phone charger. Unlike the traditional two-stage alternating current (AC)direct current (DC) regulator or voltage regulation at the transmitter by using wireless communication, a non-charging configuration is introduced to regulate the output voltage at a constant voltage with a PWM control algorithm. With this method, there is no need for the DC-DC stage after the rectifier and wireless communication circuit blocks. Besides, a full active cross-coupled rectifier with a high-speed comparator effectively reduces the power loss of the regulator. This solution is suitable for those low cost IPT application.

- Design of a high-efficiency full active rectifier for MRC wireless power transfer system at $6.78-\mathrm{MHz}$ with ATD control. For this design, an ATD control circuit is developed to expand the conduction interval of the $\mathrm{GaN}$ switch in order to reduce the power loss due to the forward voltage of the diode. The proposed control algorithm also eliminates the reverse leakage current of the rectifier, thus improving the power conversion efficiency of the rectifier. Meanwhile, the proposed rectifier is designed with the capability to self-start in the initial stage when the voltage level is low. The controller is implemented in a high-voltage complementary metal-oxide semiconductor (CMOS) process. GaN transistors are assembled with the controller on a printed circuit board (PCB). The proposed rectifier can output a maximum output current of $3 \mathrm{~A}$ at $5 \mathrm{~V}$ with a $6.78-\mathrm{MHz} \mathrm{AC}$ input voltage. The proposed rectifier can achieve a peak power efficiency of 90 $\%$.

- Design of a hybrid control method for 6.78-MHz MRC wireless power transfer system with varying transmission distance with a ZVS class-D power amplifier. The efficiency (coil to coil) of the WPT system decreases as the distance increases. So a 6.78-MHz MRC technology is used to enhance the efficiency. However, traditional MRC WPT has to work at resonant frequency over a key coupling coefficient to maintain the efficiency 
and output power. In this thesis, detailed analysis of MRC WPT, especially on the relationship between operating frequency, transfer efficiency, output power, and coupling coefficient are presented. Contrary to the traditional research, this thesis proves that the system is able to work with sufficient output power and efficiency within a wide coupling coefficient range. A hybrid control method with frequency/phase-shift tuning with ZVS Class$\mathrm{D}$ amplifier is proposed to provide the load with a constant output voltage with high efficiency in a wide range of charging distance.

\subsection{Organization of the Thesis}

This thesis consists of six chapters. Chapter 1 introduces the background of WPT and lists the research objectives and scope. Chapter 2 reviews the published literature with regard to the following: (i) the structures of WPT for different applications, (ii) several high-efficiency rectifiers for WPT and radio-frequency identification (RFID) energy-harvesting applications with full active structures, (iii) several high-performance DC to AC converters with zero voltage switch (ZVS) or zero current switch (ZCS) technology, and (iv) several WPT power control methods. A novel single-stage WPT receiver suitable for low-cost IPT WPT operation at $125-\mathrm{kHz}$ is presented in Chapter 3, followed by the design and discussion of a high-efficiency rectifier for 6.78-MHz MRC WPT application in Chapter 4. In Chapter 5, a 6.78-MHz MRC WPT system with hybrid control of frequency-shifting and phase-shifting is proposed and verified. Finally, Chapter 6 concludes the thesis and also provides suggestions for future work. 



\section{Chapter 2}

\section{Literature Review}

\subsection{Basic concepts and principle of WPT}

\subsubsection{Magnetic coupling}

Magnetic coupling in a WPT system means transfer of power through a magnetic field. The theory behind magnetic WPT is the Ampere's and Faraday's laws. According to Ampere's law, the displacement current can be neglected in an ideal case:

$$
\oint_{C_{1}} H d l=\oint_{S_{2}} J d S
$$

An AC through the primary coils will generate an alternating magnetic field over a closed contour $C_{1}$ that encloses the transmitting coils. Assuming that the currents through all turns of the transmitting coils are the same, Equation (2.1) can be simplified to give:

$$
\oint_{C_{1}} H d l=N_{1} I_{1}
$$

Equation (2.2) indicates that the magnetic field intensity depends on the number of turns of the transmitting coil and the transmitting current. Faradays law states that when the receiving coil is exposed to an alternating magnetic field, an induced voltage will be created at the receiving coil terminals: 


$$
\oint_{C_{2}} E d l=-\frac{\partial}{\partial t} \oint_{S_{2}} B d S
$$

Thus power is further delivered to the load. It is clear that the basic concept is simple, but transferring an adequate amount of power from the transmitter to the load efficiently, controllably, and safely is much more difficult. In the text below, ways to achieve this target will be discussed.

\subsubsection{Coupled-Mode Theory}

coupled mode theory (CMT) is a basic theory for analysing the power exchange between two resonant objects [19]. Based on CMT, $a_{1}(\mathrm{t})$, and $a_{2}(\mathrm{t})$ are defined as the amplitudes of two resonant objects in the time-domain field containing energies of $\left|a_{1}(t)\right|^{2}$ and $\left|a_{2}(t)\right|^{2}$, respectively . A first-order differential equation between two resonant objects is used to describe the power exchange [20] [21]:

$$
\begin{gathered}
\frac{d a_{1}(t)}{d t}=-\left(j \omega_{1}+\Gamma_{1}\right) a_{1}(t)+j K_{12} a_{2}(t)+F_{S}(t) \\
\frac{d a_{2}(t)}{d t}=-\left(j \omega_{2}+\Gamma_{2}+\Gamma_{L}\right) a_{2}(t)+j K_{12} a_{1}(t)
\end{gathered}
$$

where $\omega_{1}$ and $\omega_{2}$ are the eigenfrequencies of the two resonant objects, and $\Gamma_{1}$, $\Gamma_{2}$ are the intrinsic decay rates due to the power loss of the coil (ohmic loss) and propagation medium (radiative loss), $\Gamma_{L}$ is the decay rate of the power consumed by the load resistance connected to the receiving coil, $F_{S}(\mathrm{t})$ is the excitation applied to the transmitter, which can be treated as a power source, and $K_{12}$ is the coupling rate between the two resonant objects. Generally, in a WPT system, the two resonant objects should be tuned to the same resonant frequency, and thus $\omega_{1}=\omega_{2}=\omega$.

In steady-state analysis, it is assumed that $F_{S}(\mathrm{t})$ is a sinusoidal signal that is equal to $A_{S} \cdot e^{-j \omega t}$, so that $a_{1} t=A_{1} \cdot e^{-j \omega t}$ and $a_{2} t=A_{2} \cdot e^{-j \omega t}$. It can be derived 
from Equation (2.4) and Equation (2.5) that

$$
\frac{A_{2}}{A_{1}}=\frac{j K_{12}}{\Gamma_{2}+\Gamma_{L}}
$$

The ohmic losses of the two resonant objects are $P_{1}=2 \Gamma_{1}\left|A_{1}\right|^{2}$ and $P_{2}=$ $2 \Gamma_{2}\left|A_{2}\right|^{2}$, while the power transfer to the load is $P_{L}=2 \Gamma_{L}\left|A_{2}\right|^{2}$. The total power generated from the source $F_{S}(\mathrm{t})$ is thus $P_{S}=P_{1}+P_{2}+P_{L}$. Therefore, the power transfer efficiency of the two resonant objects can be calculated as the ratio of power delivered to the load to the power from the source.

$$
\eta=\frac{P_{L}}{P_{S}}=\frac{\Gamma_{L}\left|A_{2}\right|^{2}}{\Gamma_{1}\left|A_{1}\right|^{2}+\Gamma_{2}\left|A_{2}\right|^{2}+\Gamma_{L}\left|A_{2}\right|^{2}}
$$

\subsubsection{Maximum Power Transfer Principle and Maximum Transfer Effi- ciency Principle}

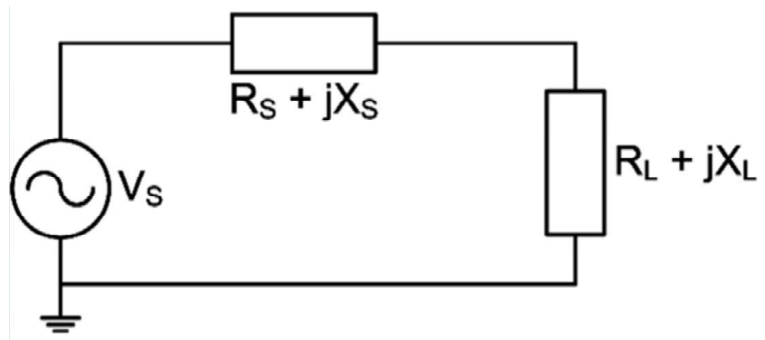

(a)

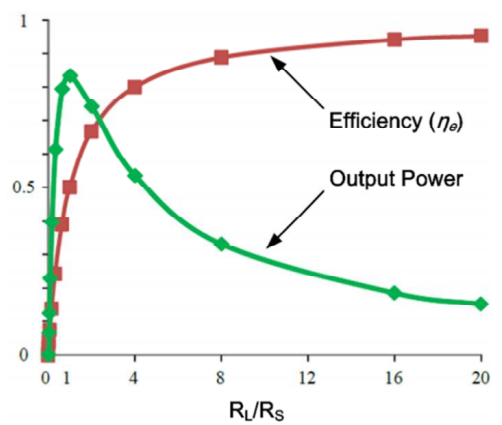

(b)

Figure 2.1: (a) Equivalent circuit of the power transfer system, (b) variations of output power and transfer efficiency as a function of $R_{L} / R_{S}$ [22].

Regardless of the structure of the power transfer system, it can be described by an equivalent circuit, as shown in Figure 2.1 (a). The maximum power transfer occurs when the source and load impedance match each other, which means that $R_{S}=R_{L}$ and $X_{S}=X_{L}$. The transfer efficiency in this condition can be derived as:

$$
\eta_{P \max }=\frac{i^{2} R_{L}}{i^{2} R_{L}+i^{2} R_{S}}=\frac{R_{L}}{R_{L}+R_{S}}=50 \%
$$

The variation of the output power and transfer efficiency as a function of the 
ratio $R_{L} / R_{S}$ is shown in Figure 2.1 (b). For fixed $R_{S}$, it is obvious that the transfer efficiency is proportional to $R_{L} / R_{S}$. So the maximum transfer efficiency occurs when equivalent load $R_{L}$ reaches its maximum allowed value $\left(R_{L\lrcorner \max }\right)$, given by

$$
\eta_{\max }=\frac{i^{2} R_{L\lrcorner \max }}{i^{2} R_{L\lrcorner \max }+i^{2} R_{S}}=\frac{R_{L\lrcorner \max }}{R_{L\lrcorner \max }+R_{S}}
$$

From the Equation (2.8) and Equation (2.9), it is concluded that the maximum output power and maximum efficiency do not have the same conditions. In a real design, the tradeoff between these two conditions is important to the designer, and will be discussed later.

\subsubsection{Topologies of WPT}

\subsubsection{Two-coil WPT}

In WPT research over recent years, a widely adopted structure is the twocoil system consisting of a transmitting coil and a receiving coil, where magnetic power is generated and exchanged between coils. Different compensation structures are used to tune the coils so that they are resonant. Figure (2.2)(a)(d) shows four basic compensation topologies, named series-series (SS), series-Parallel (SP), parallel-series (PS), and parallel-parallel (PP). Here, the relationships between the voltage and current are listed as follows:

Here,the relationships between the voltage and current are listed as follows:

For SS:

$$
\begin{gathered}
V_{1}=I_{1}\left(R_{1}+j \omega L_{1}+\frac{1}{j \omega C_{1}}\right)+I_{2} j \omega M \\
0=I_{1} j \omega M+I_{2}\left(R_{2}+R_{L}+j \omega L_{2}+\frac{1}{j \omega C_{2}}\right)
\end{gathered}
$$

For SP: 


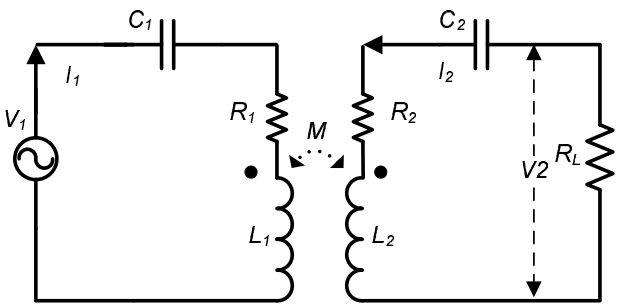

SS

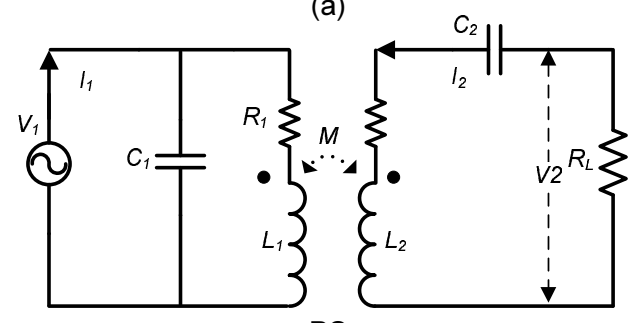

(c)

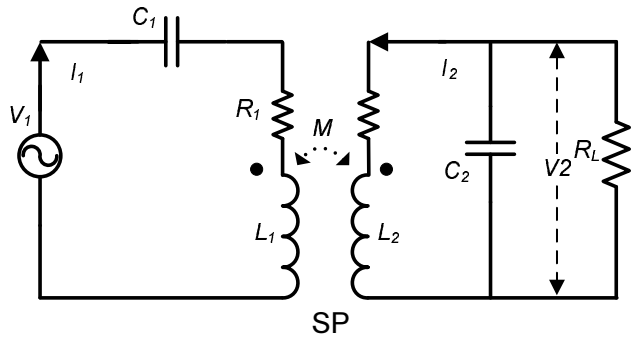

(b)

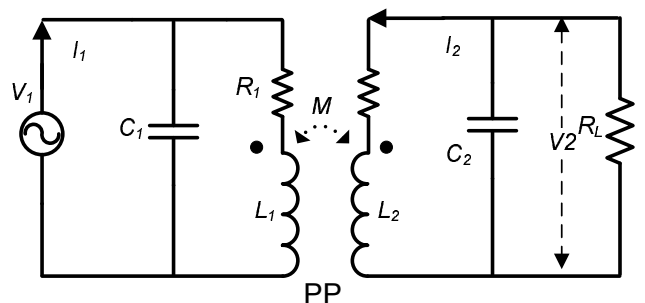

(d)

Figure 2.2: Topologies of four basic compensation network.

$$
\begin{gathered}
V_{1}=I_{1}\left(R_{1}+j \omega L_{1}+\frac{1}{j \omega C_{1}}\right)+I_{2} j \omega M \\
0=I_{1} j \omega M+I_{2}\left(R_{2}+\frac{\frac{R_{L}}{j \omega C_{2}}}{\frac{1}{j \omega C_{2}}+R_{L}}\right)
\end{gathered}
$$

For PS:

$$
\begin{gathered}
I_{1}=V_{1} j \omega C_{1}+\frac{\left(V_{1}-I_{2} j \omega M\right)}{j \omega L_{1}} \\
0=I_{1} j \omega M+I_{2}\left(R_{2}+R_{L}+j \omega L_{2}+\frac{1}{j \omega C_{2}}\right)
\end{gathered}
$$

For PP:

$$
\begin{gathered}
I_{1}=V_{1} j \omega C_{1}+\frac{\left(V_{1}-I_{2} j \omega M\right)}{j \omega L_{1}} \\
0=I_{1} j \omega M+I_{2}\left(R_{2}+\frac{\frac{R_{L}}{j \omega C_{2}}}{\frac{1}{j \omega C_{2}}+R_{L}}\right)
\end{gathered}
$$

where the mutual inductance $(\mathrm{M})$ indicates the coupling strength of the coils, and the self-inductance of the transmitting coil $\left(L_{1}\right)$, self-inductance of the re- 
ceiving coil $\left(L_{2}\right)$ indicates the energy store capabilities of both coils. The WPT system works in resonance, which means the operation frequency $(\omega)$ equals the resonant frequency of both the primary side $\left(\omega_{1}\right)$ and the secondary side $\left(\omega_{2}\right)$, which is expressed as:

$$
\omega=\omega_{1}=\omega_{2}=\frac{1}{\sqrt{L_{1} C_{1}}}=\frac{1}{\sqrt{L_{2} C_{2}}}
$$

If the system works in resonant mode and the ESR of the coils are so small that can be omitted, the relationships between the voltage and current for the secondary side are as listed below:

For series compensation:

$$
V_{2}=I_{1} j \omega M
$$

For parallel compensation:

$$
I_{2}=I_{1} \frac{M}{L_{2}}
$$

According to Equations $(2.19,2.20)$, we can find that the series compensation has a constant-voltage capability, which means that if we keep the primary current constant, the voltage across $R_{L}$ will also have a constant value, and the parallel compensation also has a current constant capability, which means that if we keep the primary current constant, the current through $R_{L}$ will also have a constant value. To decrease the voltage-ampere (VA) rating of the WPT system, designers tend to tune the input voltage and current in phase; in other words, a zero phase angle (ZPA) is expected.

According to Equation (2.10) to (2.17), the input impedance $Z_{\text {in }}$ seen from the $\mathrm{AC}$ source $V_{1}$ can be found as follows:

For SS:

$$
Z_{\text {in_SS }}=R_{1}+\frac{\omega^{2} M^{2}}{R_{2}+R_{L}}
$$

For SP: 


$$
Z_{i n \_S P}=R_{1}+\frac{\omega^{2} M^{2}}{R_{2}+j \omega L_{2}+\frac{R_{L}}{1+j \omega C_{2} R_{L}}}
$$

For PS:

$$
Z_{\text {in_PS }}=\frac{1}{\frac{1}{R_{1}+j \omega L_{1}+\frac{\omega^{2} M^{2}}{R_{2}+R_{L}}}+j \omega C_{1}}
$$

For PP:

$$
Z_{\text {in_PP }}=\frac{1}{\frac{1}{\left(R_{1}+j \omega L_{1}\right)+\frac{\omega^{2} M^{2}\left(1+j \omega C_{2} R_{L}\right)}{\left(R_{2}+j \omega L_{2}\right)\left(1+j \omega C_{2} R_{L}\right)+R_{L}}}+j \omega C_{1}}
$$

From the above equations, it can be concluded that only SS structure's ZPA conditions are not related to the mutual inductance and the load resistance. $Z_{\text {in }}$ for the SP structure varies with M. For PS and PP, $Z_{\text {in }}$ is greatly affected by both the $\mathrm{M}$ and $R_{L}$ conditions.

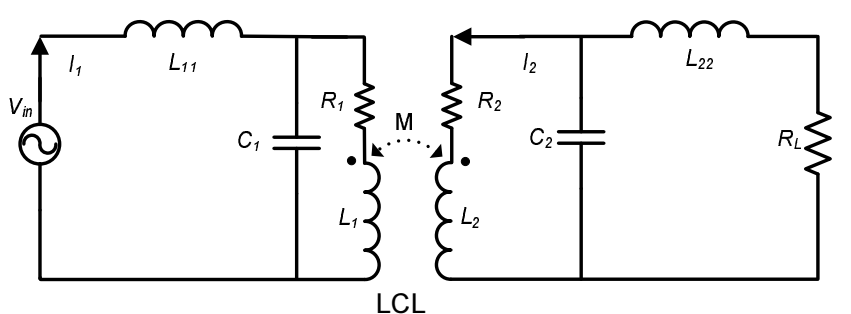

(a)

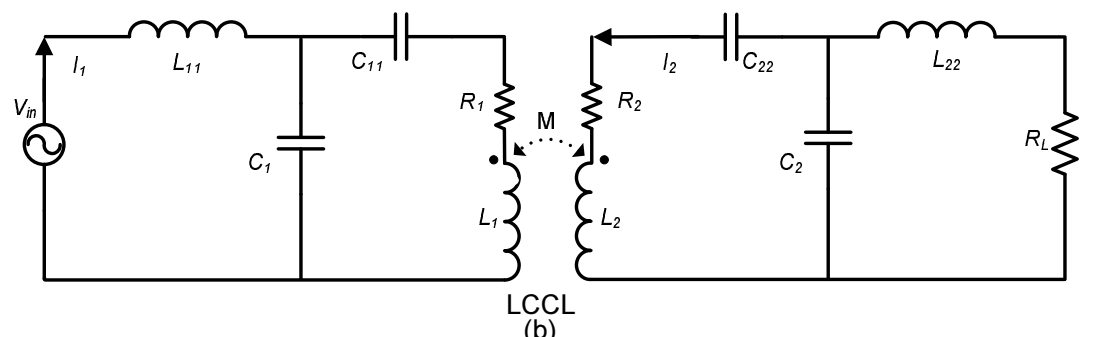

Figure 2.3: The LCL and LCCL compensation topologies.

Besides these four basic compensation techniques, [23] presents an inductorcapacitor-inductor (LCL) compensation structure (shown in Figure 2.3(a)). An SP-tuned LCL is used in the circuit to achieve a unity power factor and thus to decrease the VA rating and power loss in the coil winding, metal-oxide-semiconductor field-effect transistor (MOSFET) switches, and source resistance. Compared to 
the traditional PP compensation technique, the new LCL structure can increase the efficiency by $20 \%-30 \%$ (coil to coil), as demonstrated by the measured results illustrated in Figure 2.4.

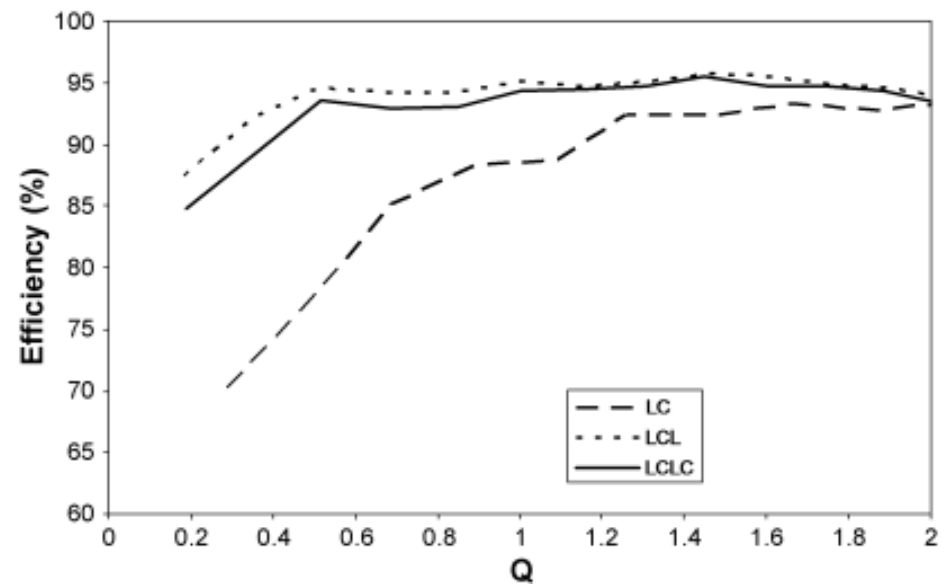

Figure 2.4: The experimental results of [23].

An SP inductor-capacitor-capacitor-inductor (LCCL) compensation (see Figure 2.3(b)) technique for WPT is presented in [24]. The author analyses the conditions for both ZVS and ZPA operation with the proposed technique. The proposed ZCS LCC compensation technique has the advantages of lower shutdown losses, lower switching losses, and faster switching transients. The results are verified with a $750-\mathrm{W}, 40-\mathrm{kHz}$ LCC compensated wireless charging system.

A brief analysis of the efficiency of the WPT system is described based on the SS compensation model. The analysis adopts a frequency-domain equivalent circuit with only the fundamental component for simplicity.

Based on Equations (2.10) and (2.11), as shown in Figure 2.5, the whole receiver could be coupled into the transmitter side as a so-called reflected impedance $Z_{r}$, which is equal to:

$$
Z_{r}=\frac{\omega^{2} M^{2}}{Z_{2}+R_{L}}
$$

where $Z_{2}$ is the impedance of the whole secondary circuit, given by:

$$
Z_{2}=j \omega L_{2}+\frac{1}{j \omega C_{2}}+R_{2}
$$




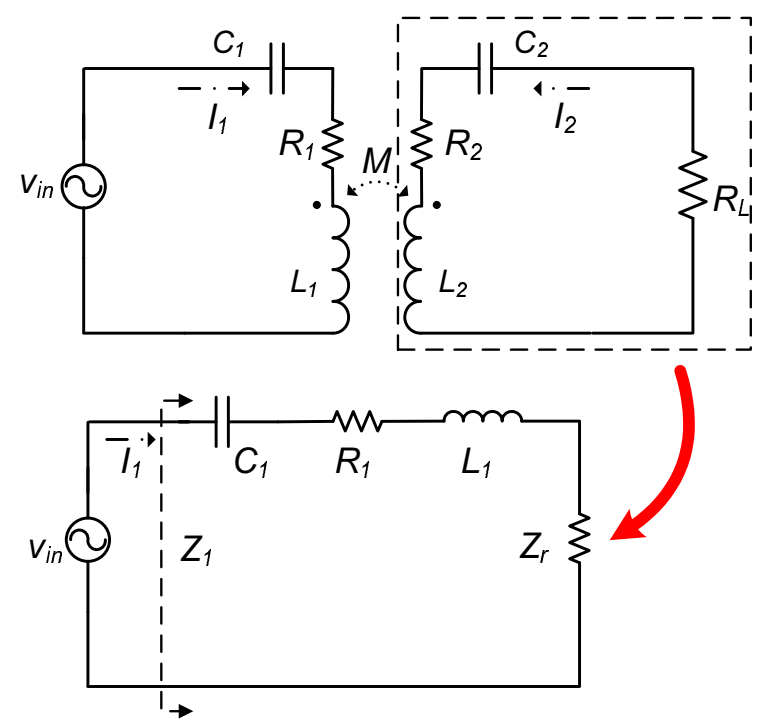

Figure 2.5: The equivalent circuit for SS model.

The power transfer efficiency is defined by considering only the real power. When the system works under resonant mode, the efficiency of the receiver side can be simply given by:

$$
\eta_{2}=\frac{R_{L}}{R_{2}+R_{L}}
$$

and the efficiency of the transmitter is:

$$
\eta_{1}=\frac{\Re\left(Z_{r}\right)}{R_{1}+\Re\left(Z_{r}\right)}
$$

in which $\mathfrak{R}\left(Z_{r}\right)$ is the real part of $Z_{r}$.

The efficiency of the whole system is the product of $\eta_{1}$ and $\eta_{2}$, which is:

$$
\eta=\eta_{1} \eta_{2}=\frac{R_{L}}{R_{1} \frac{\left(R_{2}+R_{L}\right)^{2}}{\omega^{2} M^{2}}+R_{2}+R_{L}}
$$

\subsubsection{Four-coil WPT}

Unlike the conventional two-coil system, two intermediate multi-turn coils with high $\mathrm{Q}$ values are placed between the transmitting and receiving coils. The two intermediate coils work as a repeater in the transformer and were first re- 
ported and proved at the MIT laboratory in 2007 [15]. Due to the potential of this new structure to expand the spatial freedom of the WPT system with high efficiency, it has been extensively researched [ [14], [25], [26], [27], [28], [29], and [30]]. The main disadvantage of the four-coil structure is the large space cost of the additional two coils. The high Q factor requires more turns of the coil, leading to a bigger space requirement, especially for helical coils.

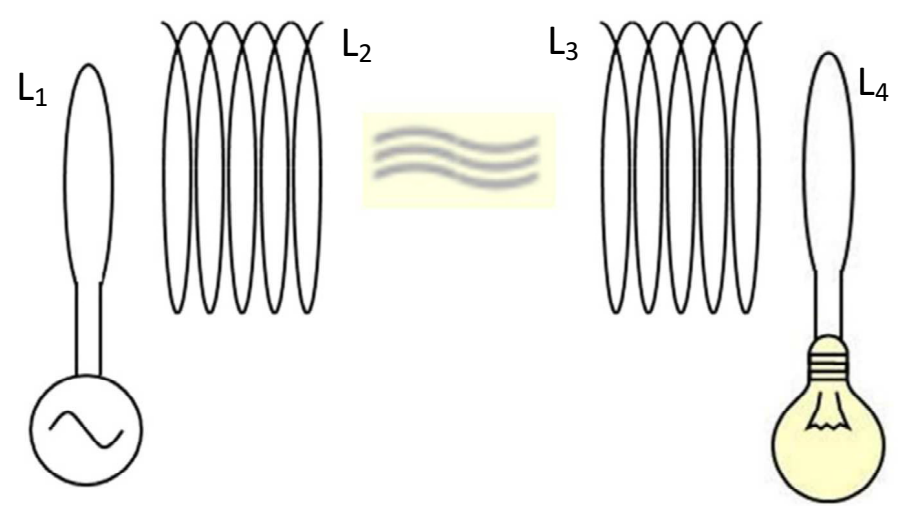

Figure 2.6: Schematic of four-coil WPT system. [31]

Figure 2.6 shows a schematic diagram of a four-coil WPT system consisting of a transmitting coil $\left(\mathrm{Coil}_{1}\right)$, receiving coil $\left(\mathrm{Coil}_{4}\right)$, and two repeaters $\left(\mathrm{Coil}_{2}\right.$, $\left.\mathrm{Coil}_{3}\right) . L_{2}$ is coupled with the transmitting coil through a high-frequency AC source that provides power to the WPT system, and $L_{3}$, coupled with the receiving coil, supplies power to the connected devices as a load. The compensation capacitors $\left(C_{1}, C_{4}\right)$ are added with the transmitting and receiving coils to make them resonant at the same frequency as the repeaters. $k_{12}, k_{23}$ and $k_{34}$ are the coupling coefficients between neighbouring coils, given by

$$
k_{i j}=\frac{M_{i j}}{L_{i} L_{j}}
$$

The couple between non-neighbouring coils is negligible in our analysis. The power transfer efficiency of the WPT system mainly depends on the coupling coefficient between the two repeaters since they are normally located a significant distance away from each other. Figure 2.7 illustrates the equivalent circuit model of the four-coil WPT system. Here, $L_{2}$ and $L_{3}$ represent the self-inductances of 


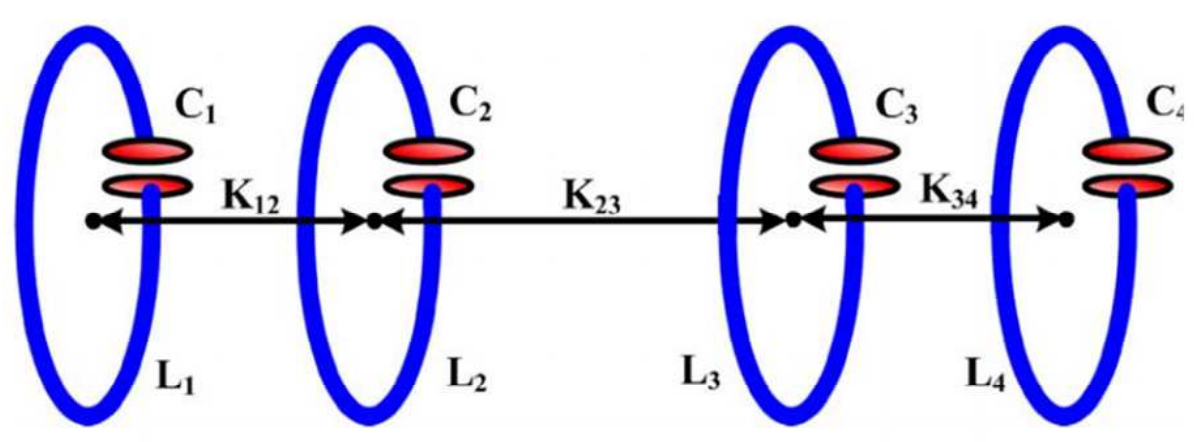

(a)

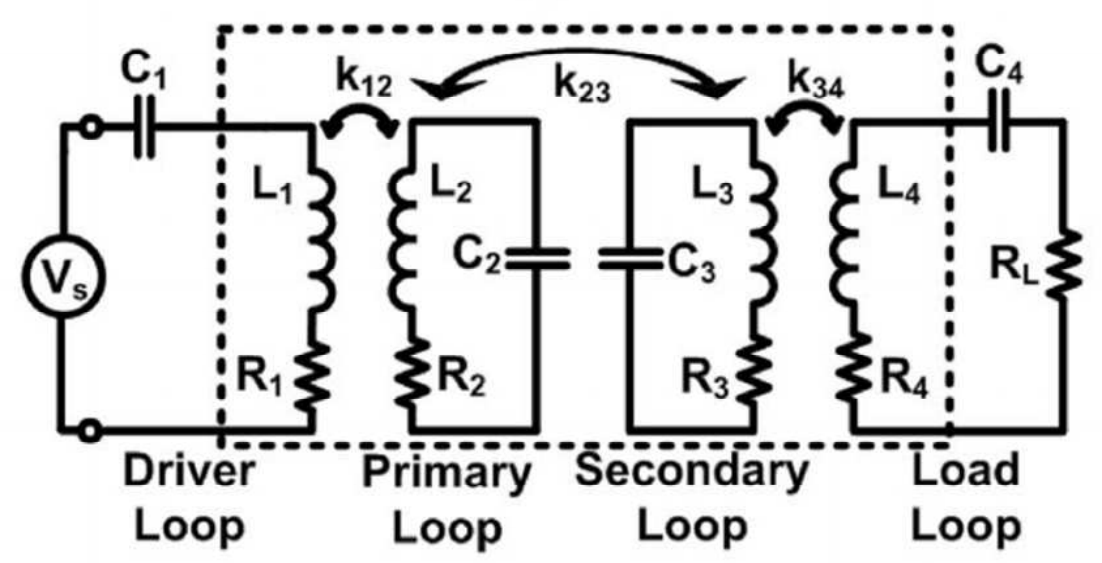

(b)

Figure 2.7: Equivalent circuit model of four-coil WPT system. [14]

the repeaters, $\mathrm{Coil}_{2}$ and $\mathrm{Coil}_{3}$, respectively, with their parasitic capacitances $C_{2}$ and $C_{3}$. Similarly, the transmitting and receiving coils are represented by $L_{1}$ and $L_{4}$, respectively. $C_{1}$ and $C_{4}$ are the compensation capacitors for $L_{1}$ and $L_{4}$. All the coils are electromagnetically coupled to each other by coupling coefficients $k_{12}, k_{23}$ and $k_{34}$. The state equations are derived and are given by:

$$
\begin{gathered}
V_{s}=I_{1}\left(j \omega L_{1}+\frac{1}{j \omega C_{1}}+R_{1}\right)+I_{2} j \omega \frac{k_{12}}{L_{1} L_{2}} \\
0=I_{2}\left(j \omega L_{2}+\frac{1}{j \omega C_{2}}+R_{2}\right)+I_{1} j \omega \frac{k_{12}}{L_{1} L_{2}}+I_{3} j \omega \frac{k_{23}}{L_{2} L_{3}} \\
0=I_{3}\left(j \omega L_{3}+\frac{1}{j \omega C_{3}}+R_{3}\right)+I_{3} j \omega \frac{k_{23}}{L_{2} L_{3}}+I_{4} j \omega \frac{k_{34}}{L_{3} L_{4}}
\end{gathered}
$$




$$
0=I_{4}\left(j \omega L_{4}+\frac{1}{j \omega C_{4}}+R_{4}+R_{L}\right)+I_{3} j \omega \frac{k_{34}}{L_{3} L_{4}}
$$

The efficiency of the four-coil system is derived in [14] and is given by:

$$
\eta_{4-\text { coil }}=\frac{\left(k_{12}^{2} Q_{1} Q_{2}\right)\left(k_{23}^{2} Q_{2} Q_{3}\right)\left(k_{34}^{2} Q_{3} Q_{4 L}\right)}{\left[\left(1+k_{12}^{2} Q_{1} Q_{2}\right)\left(1+k_{34}^{2} Q_{3} Q_{4 L}\right)\right]\left[1+k_{23}^{2} Q_{2} Q_{3}+k_{34}^{2} Q_{3} Q_{4 L}\right]} \frac{Q_{4 L}}{Q_{L}}
$$

\subsection{Control Circuits and Methods for WPT}

Designing a suitable control for WPT is important and challenging. For consumer devices, most standards [32] [33] require a constant voltage output. Some other works also control the output power, phase angle of the input voltage/current and so on. The WPT control strategies can be classified into two main categories: control at the transmitter side and the receiver. The former requires wireless communication to transfer information of control target from the receiver to the transmitter. The transmitter then can adjust inputs, like DC supply, operating frequency, phase-shift, to control the targets.

\subsubsection{Control at the transmitter side}

Qi standard is one of the mainstream WPT standards. Figure2.8 shows the basic concepts of the control in the Qi standard. This control strategy is based on backscattering communication, which is realized by changing the load of the receiver. It can be seen from Figure 2.8 that the current in the transmitter varies with the change of the load, indicating digital bit "0" or "1". As shown in Figure2.9, the realization of load changing can be resistive or capacitive, as applied in [34].

The communication circuit sends the difference between the output voltage and reference voltage back to the transmitter. The transmitter filters the signal and uses a proportional-integral-derivative (PID) control algorithm to adjust either the 


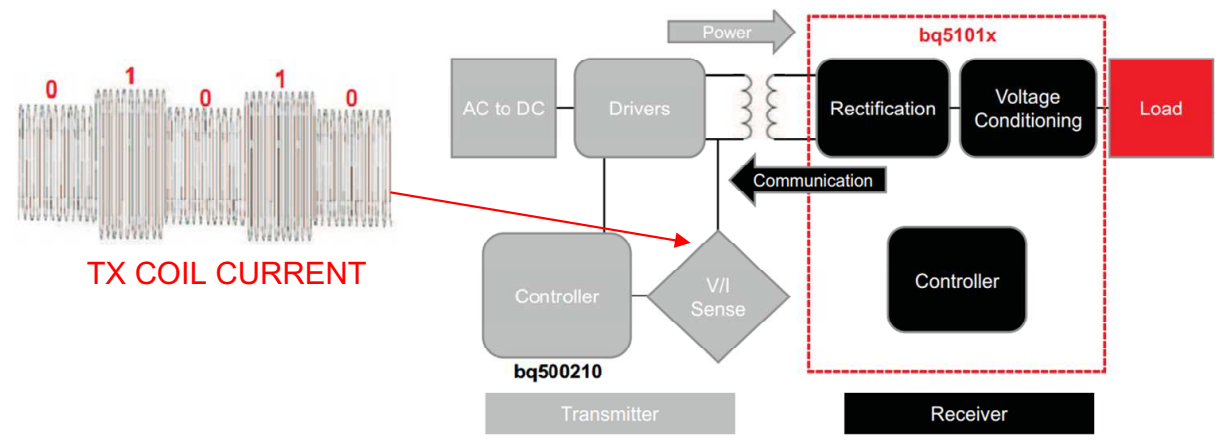

Figure 2.8: Qi wireless power transfer system [34].

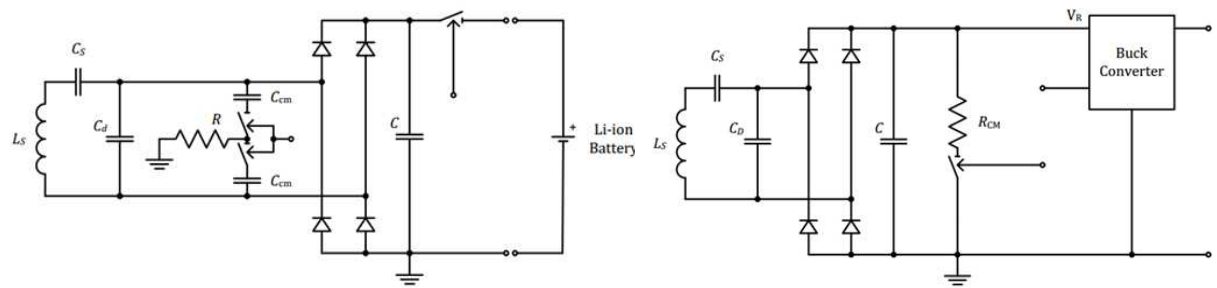

(a)

(b)

Figure 2.9: Two different kinds of communication: (a) resistive (b) capacitive [32].

duty or the frequency of the inverter, as shown in Figure 2.10.

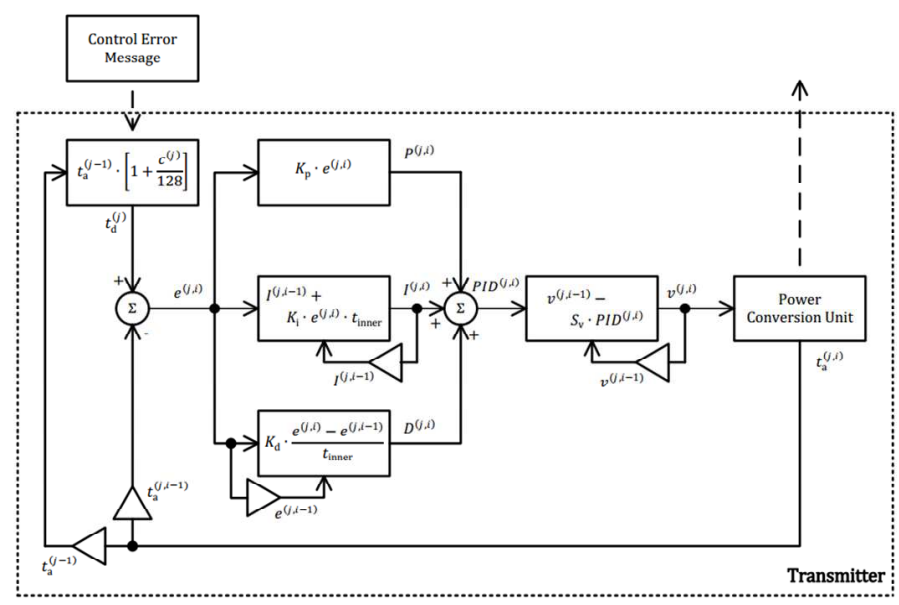

Figure 2.10: PID control algorithm [32].

A4WP is another main WPT standard. The control of the A4WP is shown in Figure 2.11. Although a DC-DC converter is used to realize fast voltage control, the A4WP system contains a blue-tooth communication module, which monitors the rectifier output for performance tracking and efficiency optimization. Since both the rectifier output and voltage across the load are constant, such control is still considered as transmitter control. 


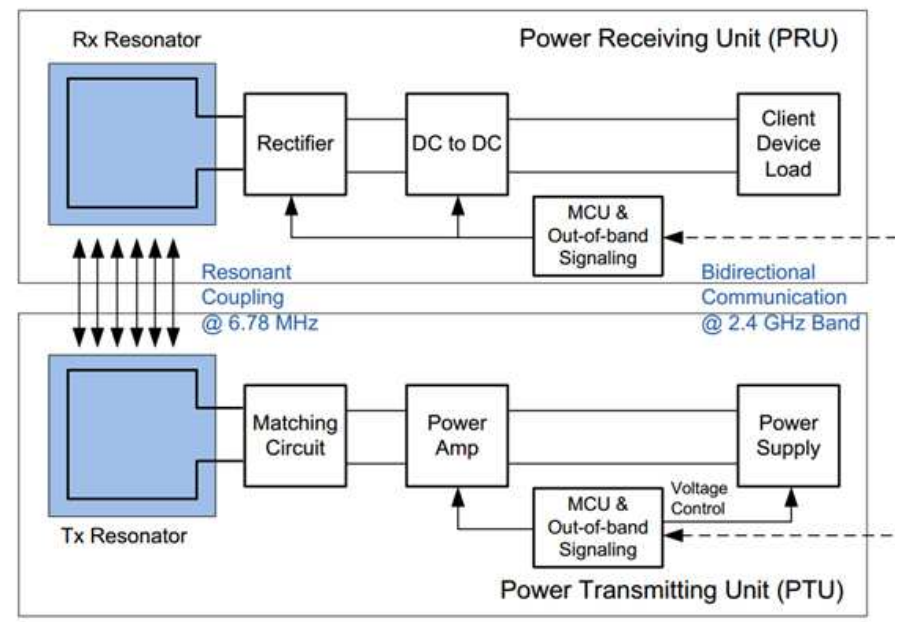

Figure 2.11: A4WP wireless power transfer system [33].

In some applications, multiple-target control is required, such as in grid-toEV WPT. Reference [12] presents a two-way WPT system for grid-EV applications. The power can be controlled bidirectionally. A theoretical model is developed to analyse both the amount and direction of power flow between EVs and grids by carrying out the control according to either the phase or the magnitude modulation of the voltages of the DC input. Figure 2.12 shows an equivalent circuit model of such a system. In this reference, the relationship between the output power of the $\mathrm{n}_{\mathrm{th}}$ load and the phase angle of $V_{\text {son }}$ and $V_{p i}$ is derived and a control system based on the equation is designed. The testing results of a $1.5-\mathrm{kW}$ prototype bidirectional WPT system with a 4-cm air gap prove the validity of the design.

\subsubsection{Control at the receiver side}

Compare to the control at the transmitter side, control at the receiver side has advantage of a faster response. The most common control circuit in the receiver side is the DC-DC converter. With regard to power management circuits on chip, there are three basic topologies for switching-mode DC-DC converters, called buck, boost, and buck-boost DC-DC converters, respectively.

- The buck converter (Figure 2.13 (a)) is utilized to provide an output voltage lower than the input voltage for applications where step-down voltage 


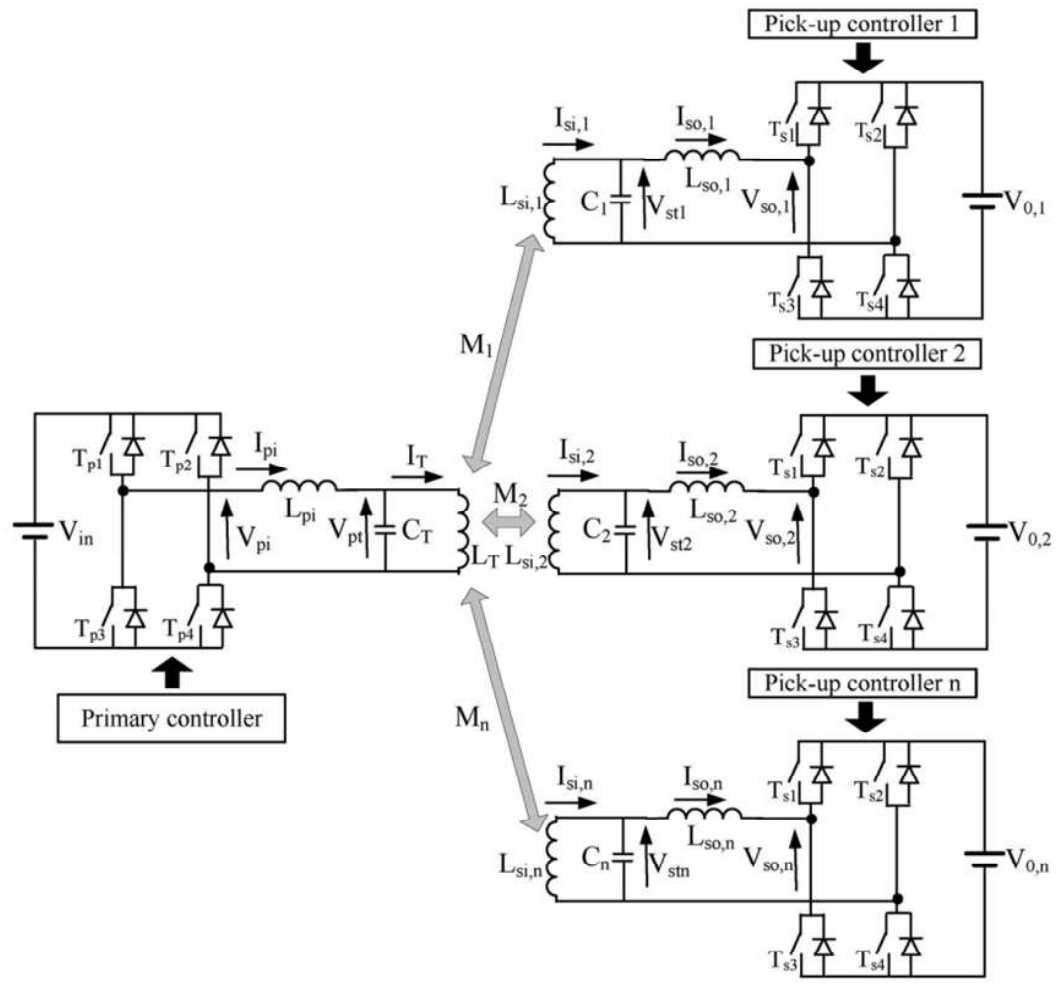

Figure 2.12: Wireless power transfer system in [12].

conversions are required.

- The boost converter (Figure 2.13 (b)) is utilized to provide an output voltage higher than the input voltage for applications where step-down voltage conversions are required.

- The output voltage of a buck-boost converter (Figure 2.13 (c)) can be either higher or lower than the input voltage for applications where both step-up and step-down voltage conversions are required.

We take the buck converter as an example to analyze the transfer function of the DC-DC converter. During the phase when $S_{1}$ is on and $S_{2}$ is off, the inductor stores energy at a rate of $\left(V_{I N}-V_{O U T}\right)$ for a duration of $\mathrm{D}$; in the phase where $S_{1}$ is off and $S_{2}$ is on, the inductor releases energy at a rate of $V_{O U T}$ for a duration of (1-D). At steady state, the energy exchange of the inductor should be equal to zero in each cycle, which leads to:

$$
D \bullet\left(V_{\text {in }}-V_{\text {OUT }}\right)-(1-D) \bullet V_{\text {OUT }}=0
$$




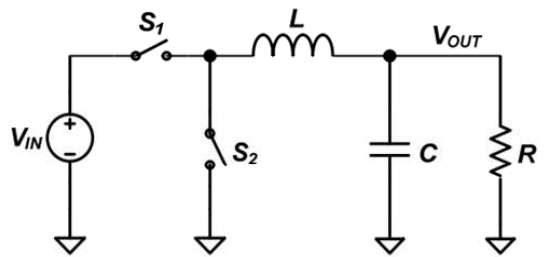

(a) Buck converter

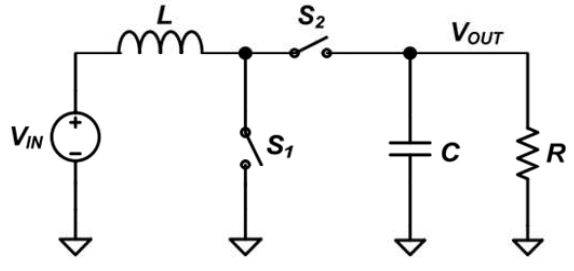

(b) Boost converter

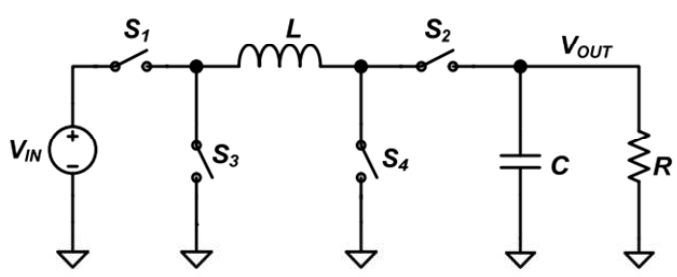

(c) Buck boost converter

Figure 2.13: Basic DC-DC Topologies.

From the above equation, the transfer function of buck DC-DC converter is given by:

$$
\frac{V_{O U T}}{V_{I N}}=D
$$

The transfer functions of the boost converter and buck-boost converter can be derived in the same way, and are given by Equation (2.38) and (Equation 2.39), respectively.

$$
\begin{aligned}
& \frac{V_{O U T}}{V_{I N}}=\frac{1}{1-D} \\
& \frac{V_{O U T}}{V_{I N}}=\frac{D}{1-D}
\end{aligned}
$$

For many applications, a stable output voltage is required in a large dynamic range of loads. So a controller is always employed. There are several control methods for DC-DC: voltage mode PWM control, current mode PWM control, pulse frequency modulation (PFM) control, and hysteretic control. Generally, the DC-DC converter works in the continuous conduction mode (CCM) and discontinuous conduction mode (DCM). 
The voltage mode PWM control adjusts the duty cycle of the DC-DC converter to maintain the output voltage with a fixed frequency. Figure 2.14 shows a typical voltage mode PWM-controlled DC-DC converter. The duty cycle can be started either by a clock signal or by the output of the comparator, as shown in Figure 2.14.

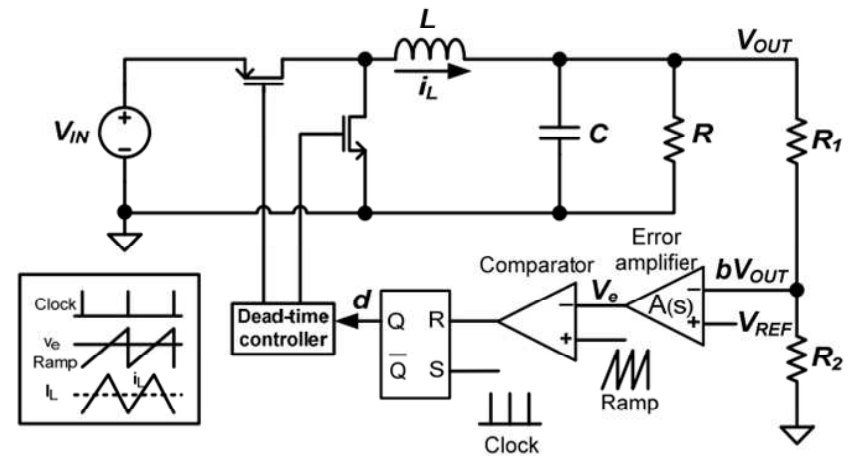

(a)
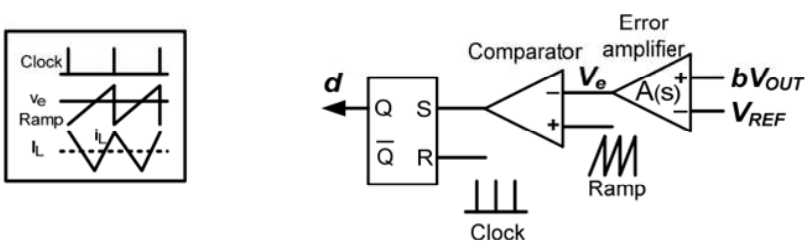

(b)

Figure 2.14: Voltage mode PWM buck DC-DC converter [35].

The current PWM-control schematic is shown in Figure 2.15. This control method can be further classified into peak current control and valley current control. Figure 2.15 shows the two different kinds of current control mode topologies. The difference between them is that the duty cycle is initiated by the clock signal in peak current mode, whereas in the valley current mode the duty cycle is initiated by the comparator signal. Generally, peak current mode is adopted in applications where the duty cycle is large $(>0.5)$, since the current sensor needs a longer response time; similarly, valley current mode is used when the duty cycle is smaller than 0.5. A slope compensation ramp is used for both modes to reduce the sub-harmonic distortion. In addition to these two modes, the average current control mode and the charge control mode are also widely used [36].

In PFM control mode, the switching frequency of the DC-DC converter varies. It is quite effective for improving the efficiency of the converter in light load con- 


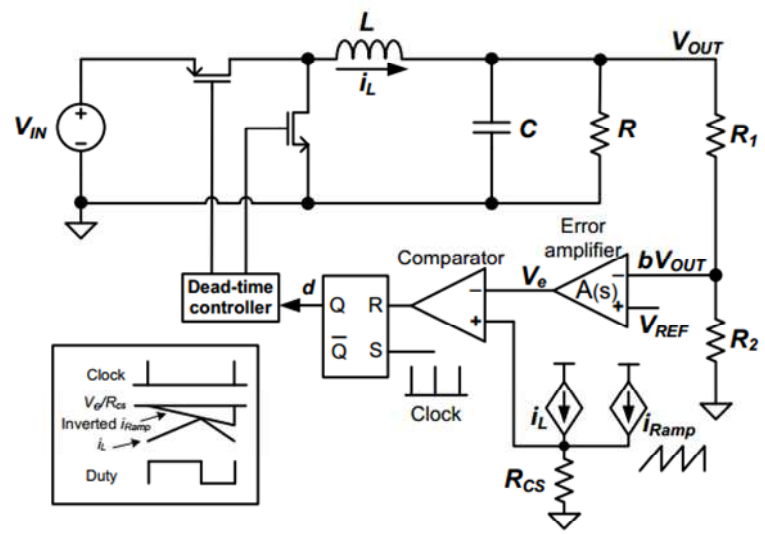

(a)

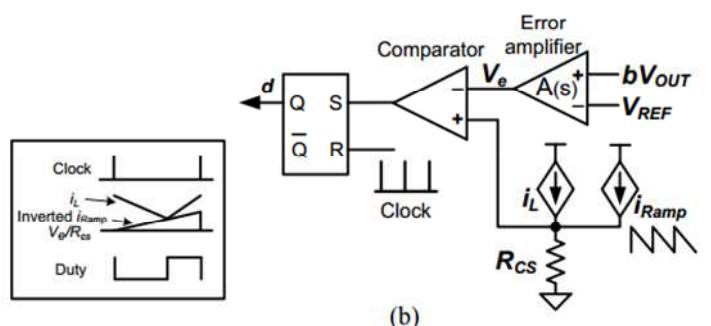

(b)

Figure 2.15: Current mode PWM buck DC-DC converter:(a) peak current control and (b) valley current mode control [35].

ditions. Figure 2.16 depicts a fixed on-time PFM mode buck converter [37]. A fixed on-time means that in each switching period, the on-time of the main switch is the same, while the switching period changes due to the difference between $V_{I N}$ and $V_{O U T}$. The other widely used PFM control strategies are fixed off-time PFM, single-pulse PFM, and multi-pulse PFM.

PFM control DC-DC has a much better conversion efficiency under light loads. However, a prominent problem of PFM mode converters is that electro magnetic interference (EMI) problems occur due to the changing switching frequency. The unpredictable frequency adds to the design complexity of the input filter. pulse skipping modulation (PSM) is thus developed to solve the existing problem. An example of this sort of converter is shown in Figure 2.17 [38]. In most PSM applications, the switching is fixed or predictable, making EMI design easier.

A hysteretic mode DC-DC converter is a special PFM. Although this kind of converter also has variable frequency, it is introduced separately here from PFM because its frequency is normally not load dependent and it is widely applied in 


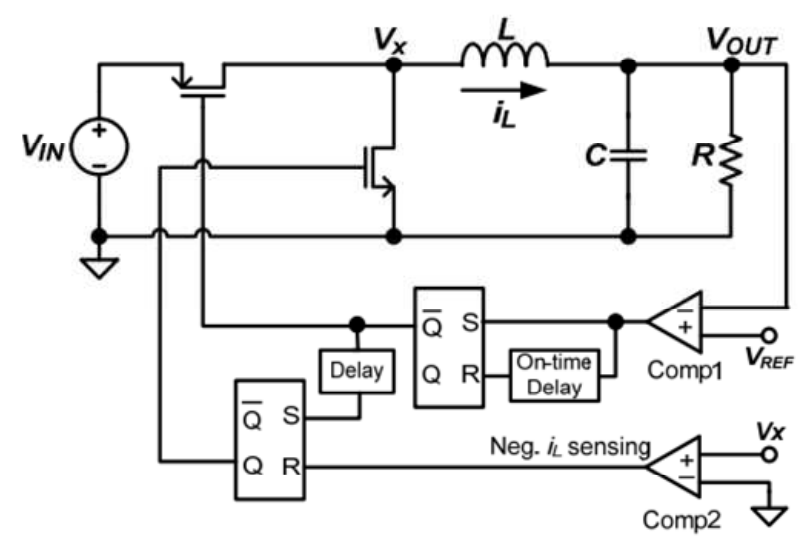

Figure 2.16: PFM control buck converter with fixed on-time control [35].

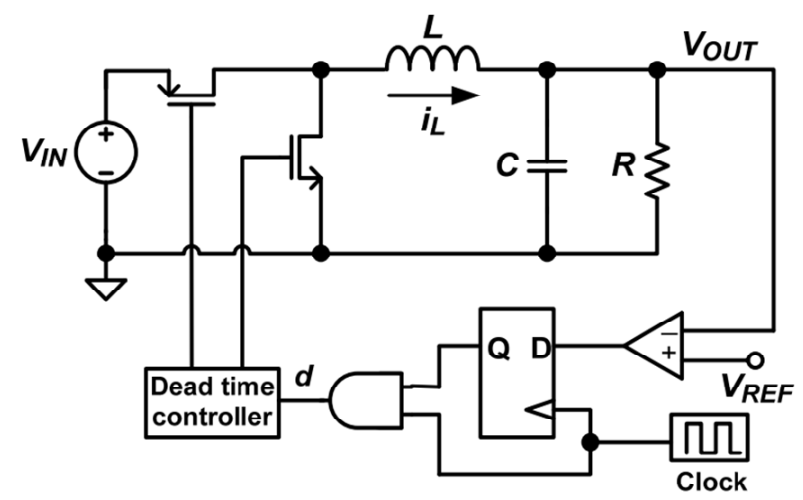

Figure 2.17: PSM control buck converter in [38].

CCM operation.

Figure 2.18 [39] shows a structure for voltage mode hysteretic control. The difference between $V_{b 1}, V_{b 2}$ and $V_{C}$ decides the mode in which the converter works.

Several DC-DC converters have been developed for WPT systems in recent years.

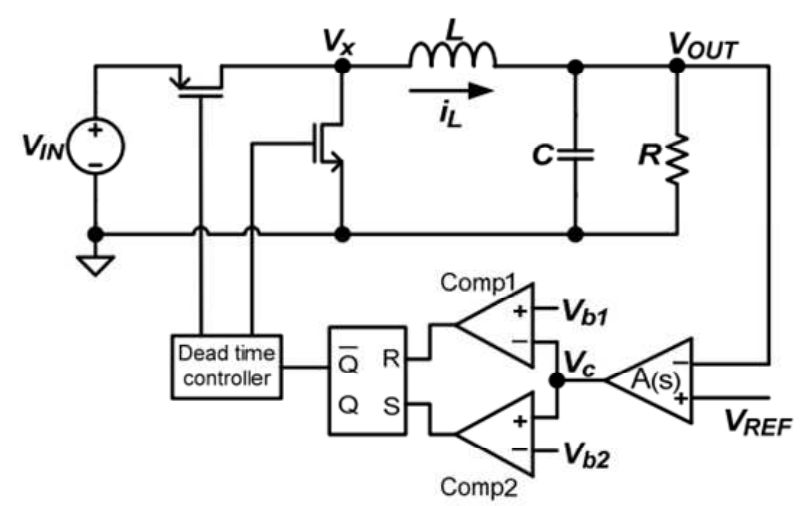

Figure 2.18: Hysteretic control DC-DC converter in [39]. 


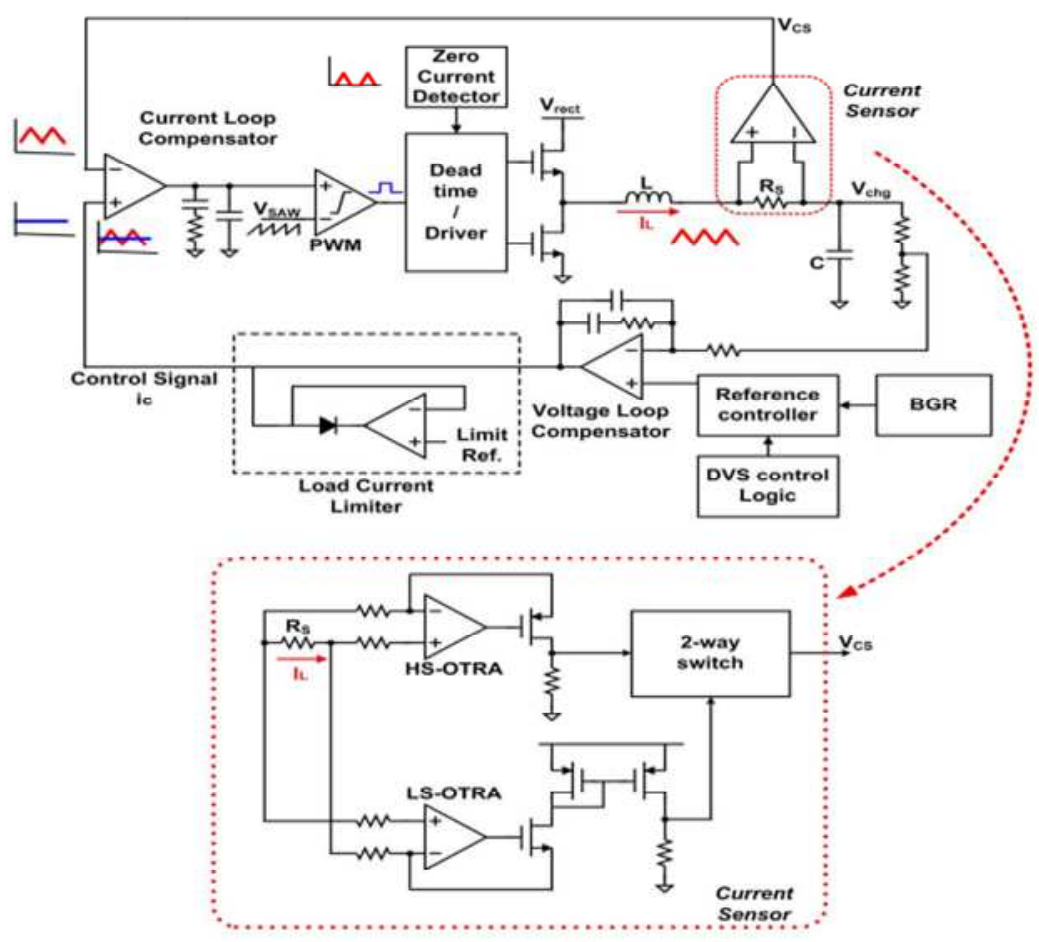

Figure 2.19: An average-current mode DC-DC with PSM for a 6 W WPT in [40].

Figure2.19 [40] shows an average current-mode converter for a 6-W output WPT application. Average current mode is adopted due to its better noise immunity, absence of extra slope compensation, accurate regulation, and good performance in both CCM and DCM modes. These benefits make this kind of converter a good choice for WPT with a DC input from the rectifier. To improve the performance in light load conditions, the PSM control mode is adopted. As a result, the efficiency of the converter is over $90 \%$ for the whole range of load conditions.

Reference [41] presents a DC-DC hysteretic buck converter with a Phase Locked Loop (PLL) (Figure 2.20). The implanted PLL helps the DC-DC to maintain the operating frequency in a large dynamic range of process, voltage and temperature (PVT) variations. Besides, with adaptive control of the PWM/PFM mode, the efficiency of the converter can be maintained from full current condition to light current condition. The maximum efficiency of the converter can reach $92.7 \%$ and the maximum of DC-DC converter is $6 \mathrm{~W}$.

There are also some other receiver voltage control circuits except for DCDC converter. Reference [42] presents an active reconfigurable voltage recti- 


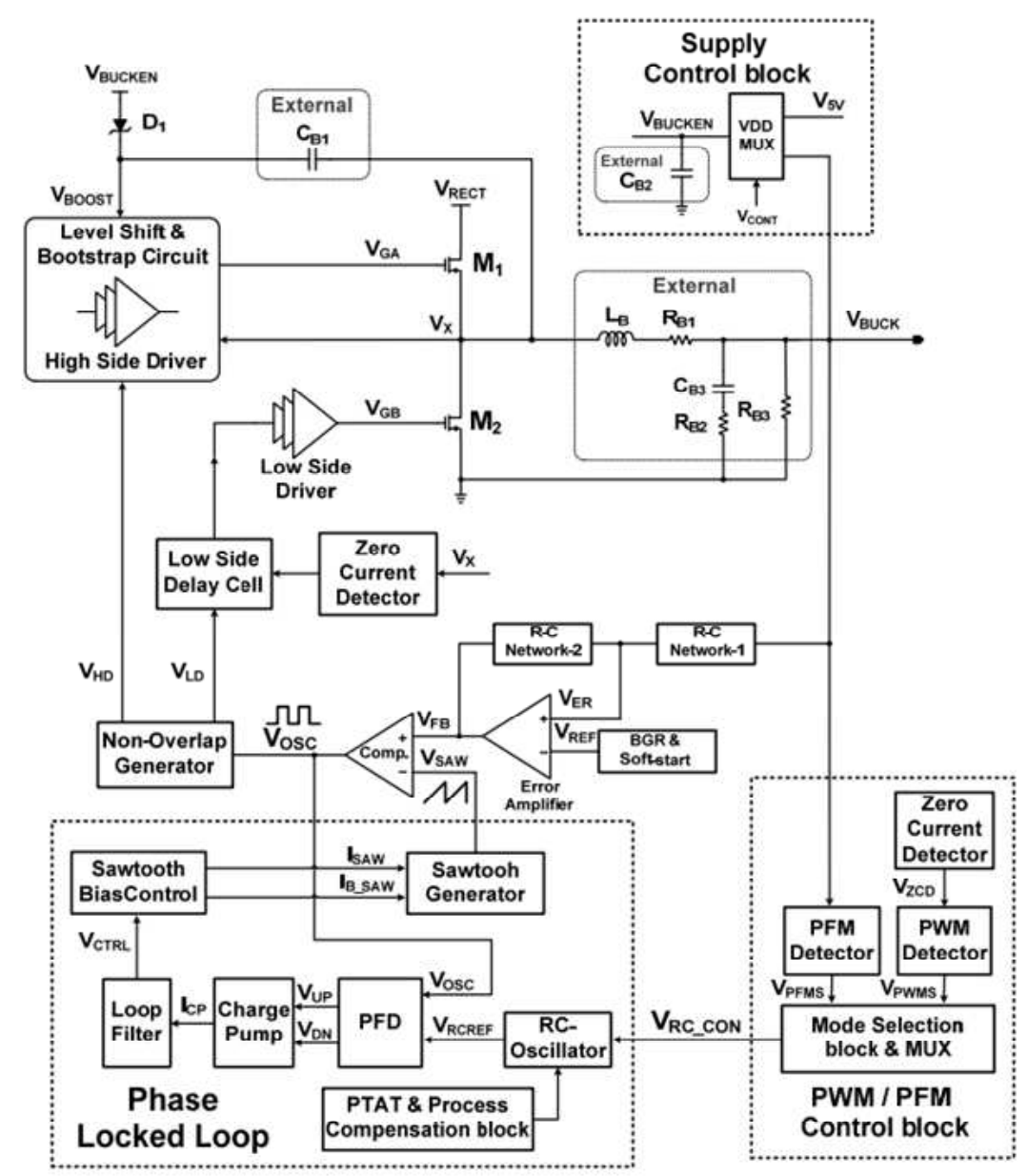

Figure 2.20: A hysteretic mode DC-DC with PLL for a $6 \mathrm{~W}$ WPT in [41].

fier/doubler rectifier for wireless power transfer of implantable microelectronic devices. The circuit model is shown in Figure 2.21. This circuit has high power conversion efficiency because both the rectifier and the voltage doubler employ active synchronous switches with low drop-out. Meanwhile, the circuit can switch between rectifier mode and voltage mode automatically according to the optimized output voltage to realize a wider range of coil arrangements with an extra output-voltage sensing circuit. This circuit has a measured power conversion efficiency (PCE) of $77 \%$ with an output voltage of $3.1 \mathrm{~V}$ and load of $0.5 \mathrm{k} \Omega$.

Reference [43] presents a rectifier and a switched capacitor converter for 6.78$\mathrm{MHz}$ high-frequency wireless charging applications, named the resonant regulating rectifier, which is an inductorless switching converter. Figure 2.22 shows the basic circuit topology. Structurally, this rectifier consists of a full diode bridge rectifier and three MOSFET switches. The key of the rectifier switching sequence 


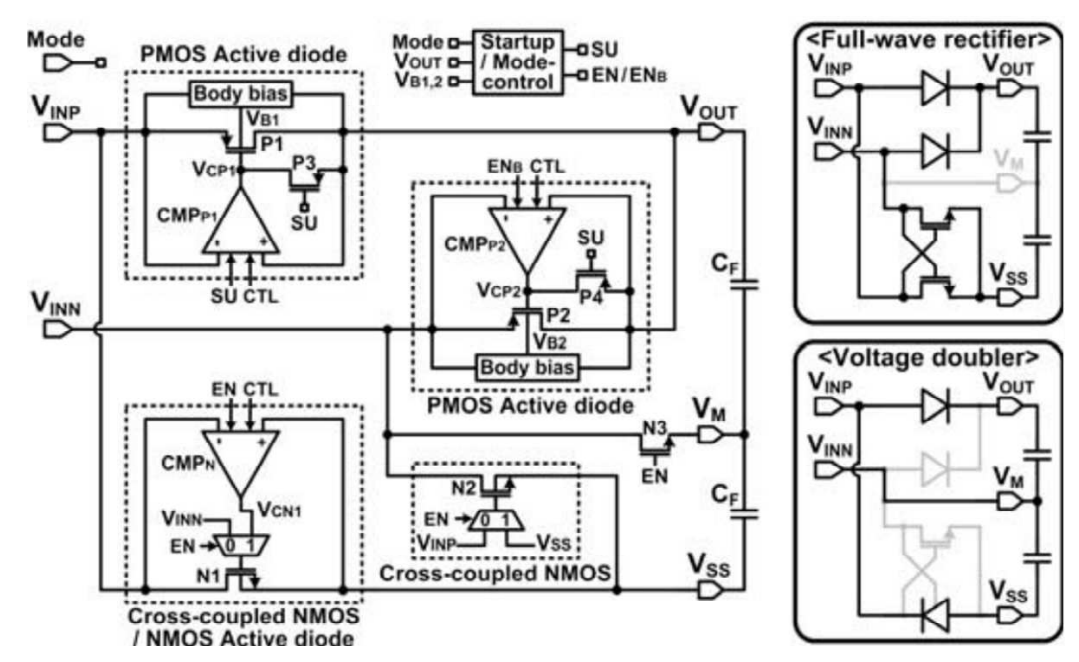

Figure 2.21: The schematic of active reconfigurable voltage rectifier/doubler rectifier in [42].

is to control the output voltage of the diode bridge up and down every eight resonant cycles with integral cycle mode, where on or off switching is determined on the output voltage. The efficiency of the circuit reaches $86 \%$ at an output power of $3.4 \mathrm{~W}$. The idea of the proposed $3 \mathrm{R}$ is to control $V_{X H}$ in Figure 2.22 between the on-duty state and the off-duty state. During the on-duty state of the circuit, $M_{2}$ is turned off and both $M_{1}$ and $M_{3}$ are turned on. For this state, the circuit is configured as a full-bridge rectifier with a smoothing capacitor consisting of $C_{F L}$ and $C_{O U T}$ connected in parallel. During the off-duty state, $M_{2}$ is turned on and both $M_{1}$ and $M_{3}$ are turned off. So the load of the circuit is switched to $C_{F L}$ in series with $C_{O U T} \|$ load. The energy and current of the receiver change consequently. PWM voltage control is used to control the circuit operating between the two states to maintain the output voltage at $5 \mathrm{~V}$.

Reference [44] reported a $13.56 \mathrm{MHz}$ WPT receiver consisting of a $1 \mathrm{X} / 2 \mathrm{X}$ reconfigurable rectifier with voltage regulation for biomedical implants. The highlight of this research is that the receiver control the voltage in two control loops: 1) a PWM loop at the receiver side controls the duty cycle of the receiver between the so called $1 \mathrm{X}$ and $2 \mathrm{X}$ modes; and 2) an outside control loop with wireless communication with the information on the ratio of $1 \mathrm{X}$ to $2 \mathrm{X}$, and thus the power of the transmitter is adjusted according to load and coupling variations. Two dif- 


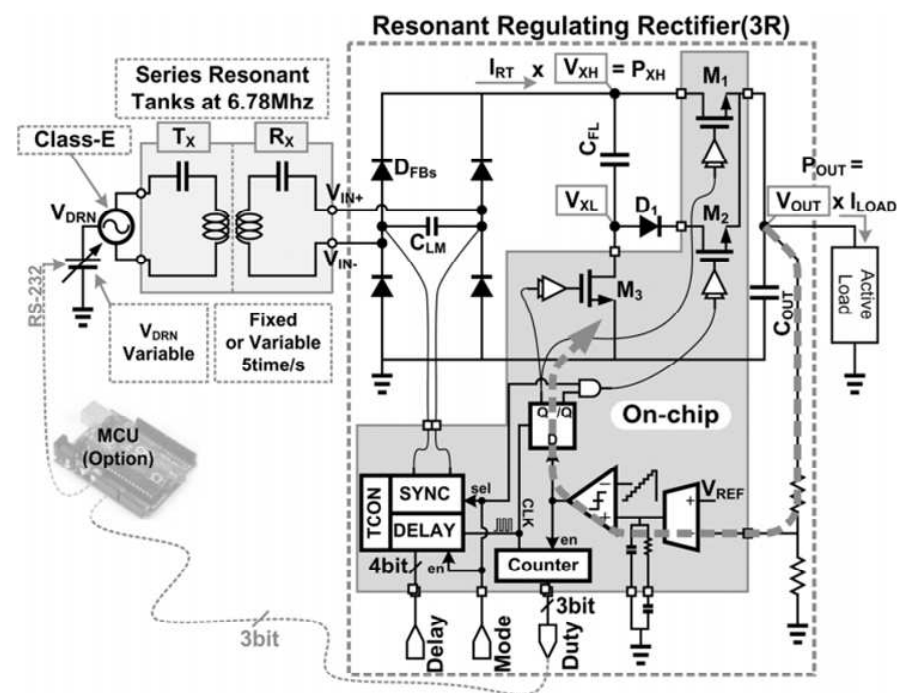

Figure 2.22: The schematic of the 3R rectifier in [43].

ferent communication techniques with backscattering method are presented for a fast response and energy-saving purpose. The transmitter and receiver of the WPT are fabricated in $0.35 \mu \mathrm{m}$ CMOS process. The digital circuits are implemented using field-programmable gate array (FPGA). The measured maximum output power is $102 \mathrm{~mW}$ and the maximum receiver efficiency is $92.6 \%$. For load transients, the overshoot and the undershoot are approximately $110 \mathrm{mV}$ and the settling times are less than $130 \mu$ s.

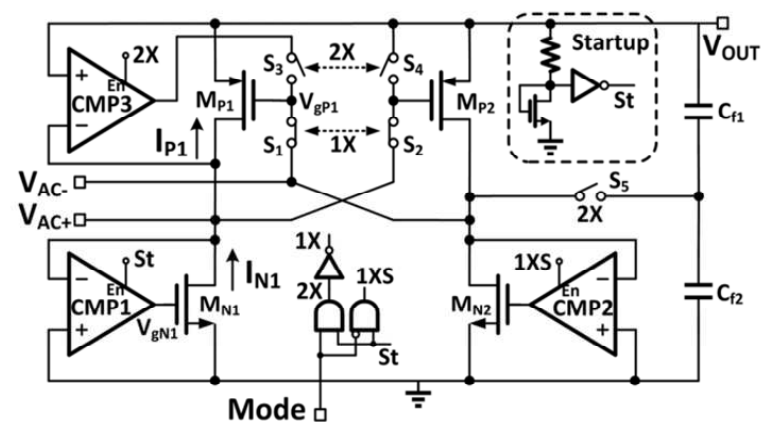

(a)

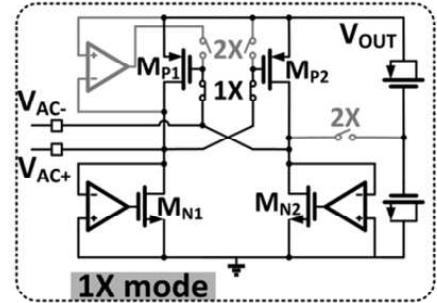

(b)

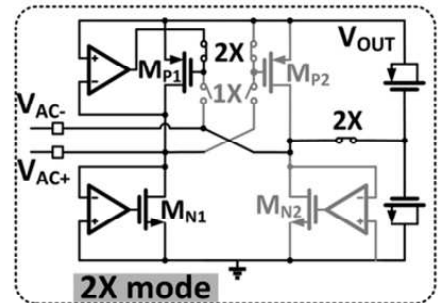

(c)

Figure 2.23: The circuit model of the $1 \mathrm{X} / 2 \mathrm{X}$ reconfigurable rectifier in [44]. 
Except voltage control in the receiver side. In some applications, the phase angle is controlled to tune the WPT system at resonance. Reference [45] proposes an active tuning method with a tri-state boost converter for a parallelcompensated secondary side circuit. Both active power and reactive power can be controlled by discontinuous current PWM modulation with the novel converter by synchronizing the switching period within the resonant period of the WPT system. A detailed circuit model of the design is shown in Figure 2.24.

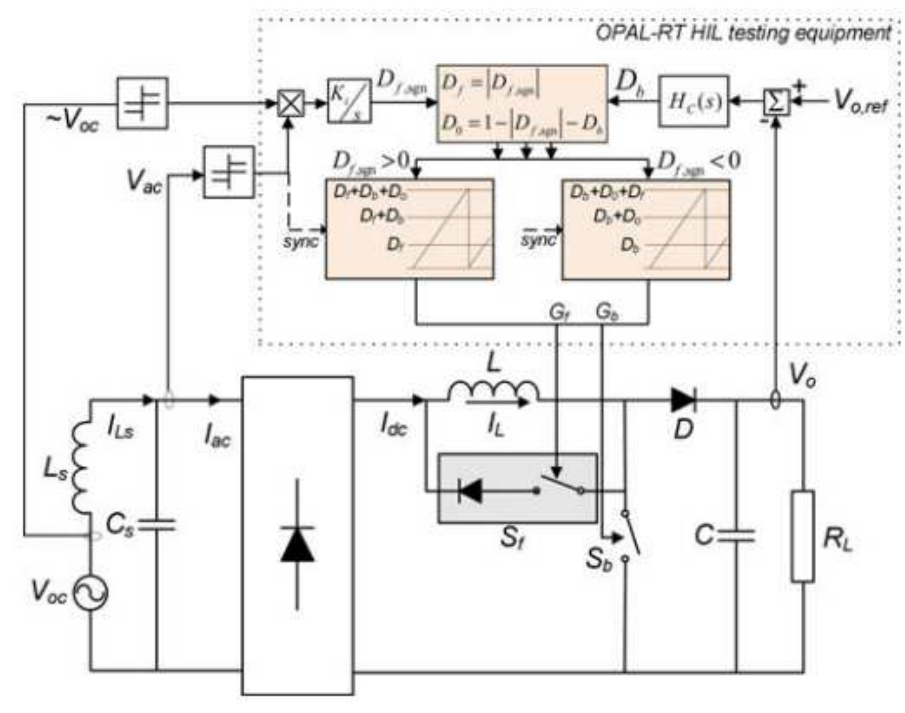

Figure 2.24: The circuit model of the tri-state boost converter in [45].

\subsection{High efficiency rectifier design}

The rectifier is a circuit that converts $\mathrm{AC}$ voltage to a $\mathrm{DC}$ voltage. In a wireless power transfer system, the rectifier regulator is mainly used in the receiver to change the induced voltage to supply the post-stage circuits. The efficiency of the rectifier is a vital consideration in WPT system design since it is related to the heat emission of the portable electronic devices, which should be restricted in a strict range. A traditional diode bridge rectifier suffers from loss caused by the forward voltage of the diodes, so it is not considered suitable for applications discussed in this thesis. Full-active rectifier, which means that the diodes would be replace by the transistor and the controller and driving circuits. Since the on-resistance of a transistor is usually small, the power loss on the transistor is much smaller than 
the diode. However, the active rectifier has an extra loss source-reverse current. As shown in Figure 2.25, $N_{1}-N_{4}$ are four transistors of the rectifier, in the positive half period, $N_{1}$ and $N_{4}$ are turned on. If the $N_{1}$ and $N_{4}$ are failed to turned off in time. The current will flow from $V_{R E C}$ to $V_{A C}$, which incur reverse leakage loss. Several kinds of rectifiers have been presented by researchers to improve the efficiency by reducing or eliminating the reverse current.
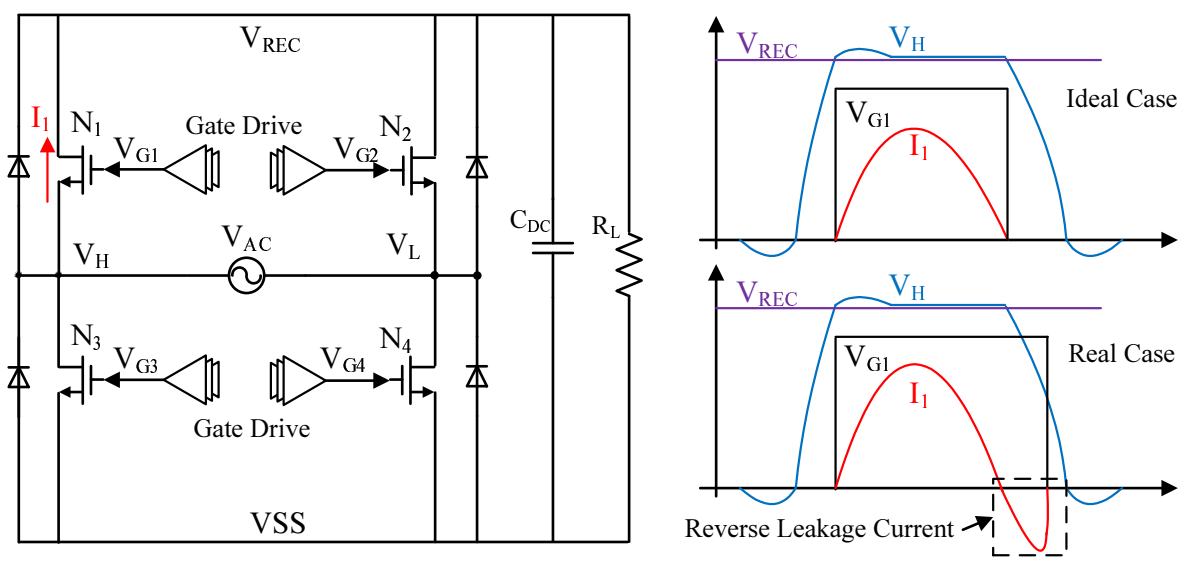

Figure 2.25: The explanation of reverse current.

(a)

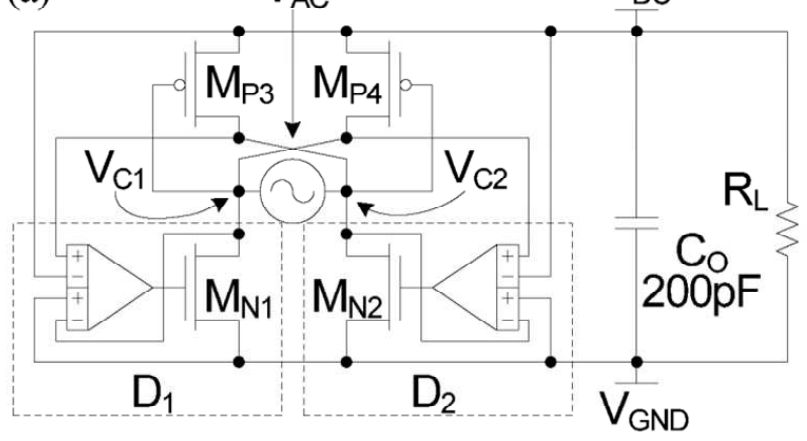

(b)

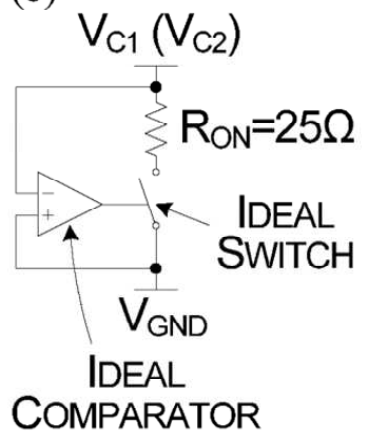

Figure 2.26: The circuit model of [46].

Reference [46] presents an active cross-connection full wave bridge rectifier for wireless power transfer in $13.56 \mathrm{MHz}$ RFID applications. The circuit consists of two cross connection PMOS and two symmetrically placed NMOS active diodes (shown in Figure 2.26) which are dynamically biased and controlled by a fast voltage comparator with reverse-current control to decrease the loss. The comparator with a 2-ns delay is a four-input circuit with a common gate structure input stage that can drive the NMOS switch working as a diode without forward 
voltage.

The proposed rectifier with an output power of $5.7 \mathrm{~mW}$ has an efficiency as high as $85 \%$. The circuit is fabricated in a $0.35 \mu \mathrm{m}$ CMOS process with an active area of $0.0055 \mathrm{~mm}^{2}$.

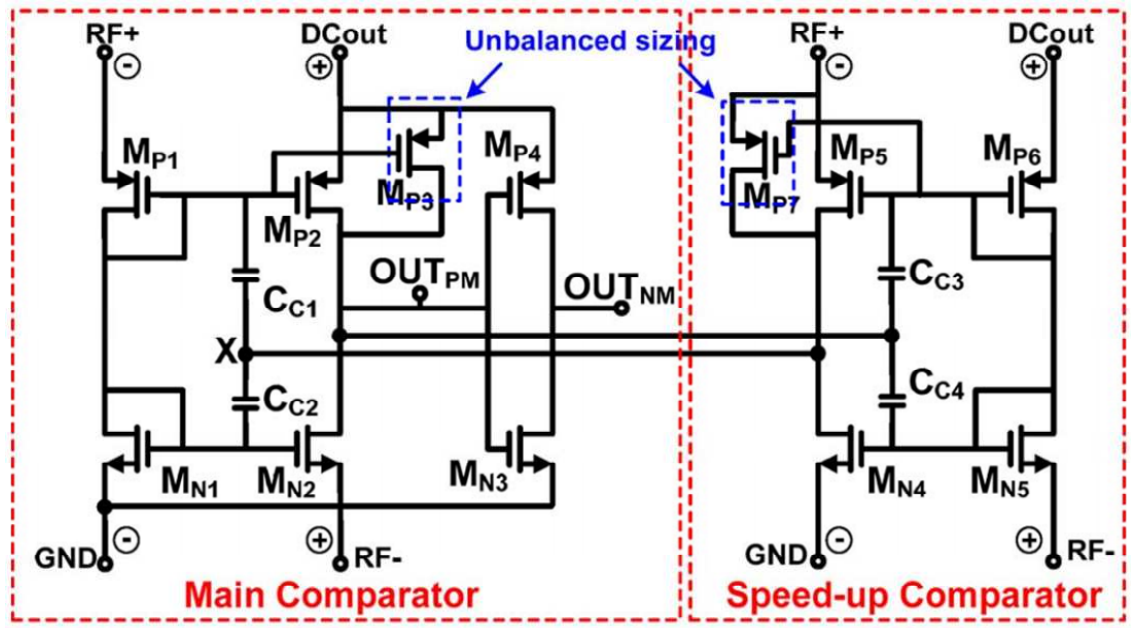

Figure 2.27: The schematic of active rectifier with speed-up latch in [47].

In [47], a highly efficient rectifier application with active MOSFETs using a speed-up common-gate-type cross-coupled latched comparator is presented for biomedical implant applications. Compared to the design in [46], the proposed rectifier introduces an unbalanced transistor and an additional comparator to accelerate the turn-on/turn-off speed of the MOSFETs. As shown in Figure 2.27, when the voltage potential of $\mathrm{RF}+$ drops below DCout, $M_{P 2}$ is turned $\mathrm{ON}$, while $M_{N 2}$ is turned OFF, thus pulling the $O U T_{P M}$ to DCout. An additional pMOSFET $M_{P 3}$ is connected parallel to $M_{P 2}$ for to enhance the pull-up effort, leading to a short response time, which minimize the leakage current.

To accelerate the transient response of the comparator, a speed-up comparator is placed parallel to the main comparator, and are coupled to the main comparator with $C_{C 1}, C_{C 2}, C_{C 3}$ and $C_{C 4}$. when $\mathrm{RF}+$ increases above DCout, $M_{P 5}$ is $\mathrm{ON}$, whereas $M_{N 4}$ is OFF, pulling the node "X" to high voltage, which speeds up the turn-on action of $M_{N 2}$ and turn-off action of $M_{P 2}$. A similar process happens when RF+ drops below DCout, turning $M_{N 4} \mathrm{ON}$ while turning $M_{P 5} \mathrm{OFF}$, pushing "X" to ground. Such actions help to turn $M_{P 2} \mathrm{ON}$ harder, contributing to a quicker 
pull-up of $O U T_{P M}$. The designed rectifier achieves a maximum measured PCE of $81.9 \%$ at $13.56 \mathrm{MHz}$ under conditions of a low $1.5-V_{p p}$ RF input signal with a $1-\mathrm{k} \Omega$ output load.

However, the solution in [47] cannot eliminate the reverse current since the comparator will not respond until the input $\mathrm{AC}$ voltage goes across the output DC voltage. A rectifier with unbalanced-biased comparators has been designed in [48]. The circuit schematic of the rectifier is shown in Figure 2.28.

The highlight of the research in [48] is that the trigger voltage for turning off the nMOSFETs $\left(M_{N 1}\right.$ and $\left.M_{N 2}\right)$ of $0 \mathrm{~V}$ is replaced with $-\Delta \mathrm{V}$. This design helps the comparator to turn off the transistors before the AC input drops below DC output, eliminating the reverse current as a result. Figure 2.28(b) depicts the scheme of the unbalanced comparator, which comprises two pairs of commongate current amplifiers CGA1 and CGA2, a current mirror $\left(M_{9}\right.$ and $\left.M_{13}\right)$, and a gate driver $\left(M_{14}, M_{15}, M_{16}\right.$, and $\left.M_{17}\right)$. The unbalanced biasing is created by setting $I_{S 1}>I_{S 2}$ and $\Delta \mathrm{V}$ is given by:

$$
\Delta V=\frac{\sqrt{I_{S 1}}-\sqrt{I_{S 2}}}{2 \sqrt{\alpha}}
$$

where $\alpha$ is given by:

$$
\alpha=(1 / 2) \mu_{n} C_{O X}(W / L)
$$

and (W/L) is the size of the transistors in $C G A_{1}$ and $C G A_{2}$. When $V_{\text {in } 2}$ rises above - $\Delta \mathrm{V}$, the current of $M_{7}$ is mirrored to $M_{9}$, while current of $M_{10}$ is mirrored to $M_{8}$, generating a push-pull effect at node "A". In a conclusion, by carefully designing $I_{S 1}$ and $I_{S 2}, M_{n 2}$ can be fully turned off at $0 \mathrm{~V}$, thus removing the reverse leakage current. The proposed circuit is able to provide a maximum output current of $20 \mathrm{~mA}$ and operate properly with inputs of different amplitudes and frequencies. With a $1.5 \mathrm{MHz}$ input of $1.2 \mathrm{~V}$ amplitude, the proposed rectifier can achieve the peak voltage conversion ratio of $95 \%$ and the power efficiency of at least $82 \%$. 


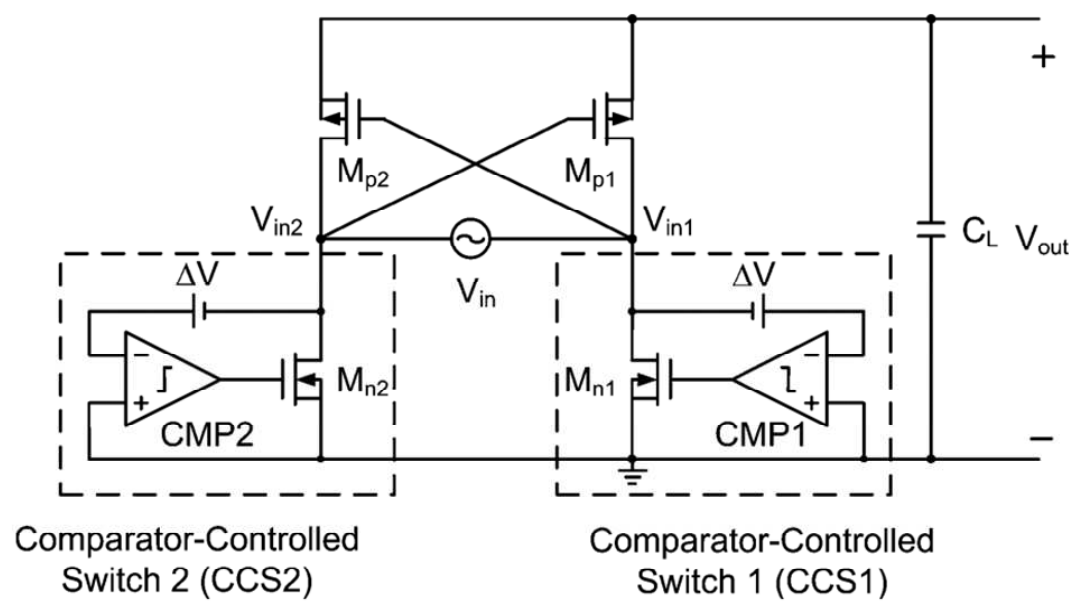

(a)

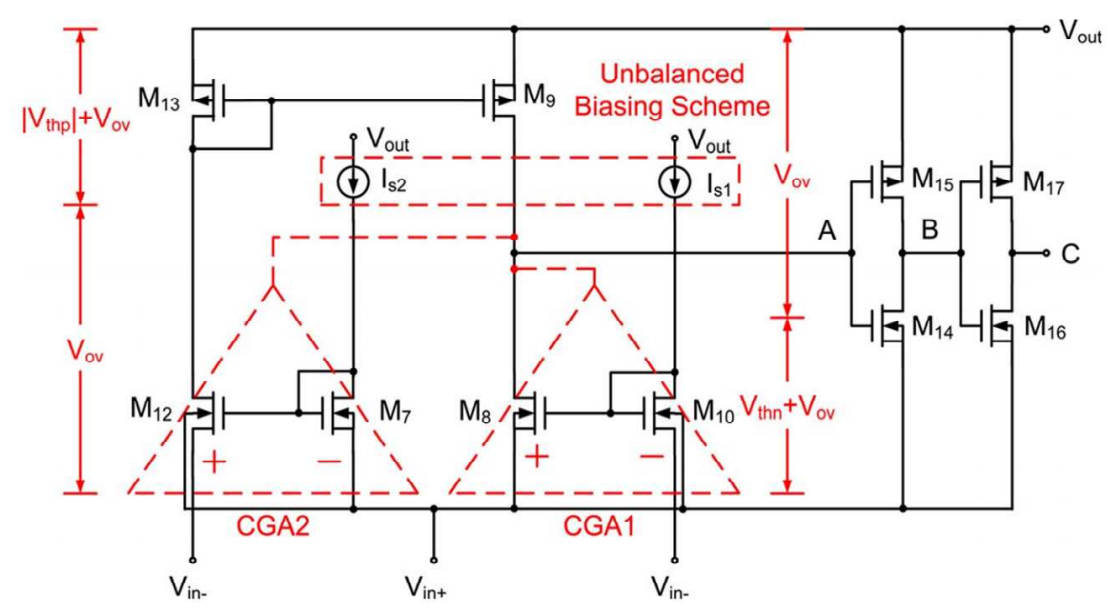

(b)

Figure 2.28: The schematic of the rectifier with unbalanced biasing design in [48].

Reference [41] reported a high-efficiency active rectifier with a delay locked loop (DLL) for $6.78 \mathrm{MHz}$ WPT applications. The schematic is shown in Figure 2.29 .

The idea of a high-speed comparator is suitable only for those applications with small output currents, for example in the range of tens of millamperes to hundreds of millamperes. For wireless power transfer in mobile phones or tablets, the large output current needs be handled by large-sized power transistors, which lead to large input capacitance of the transistors. As a result, the comparator cannot turn off the upper-side transistor in a short given time due to the large capacitance. Consequently, the power loss due to such a leakage cur- 


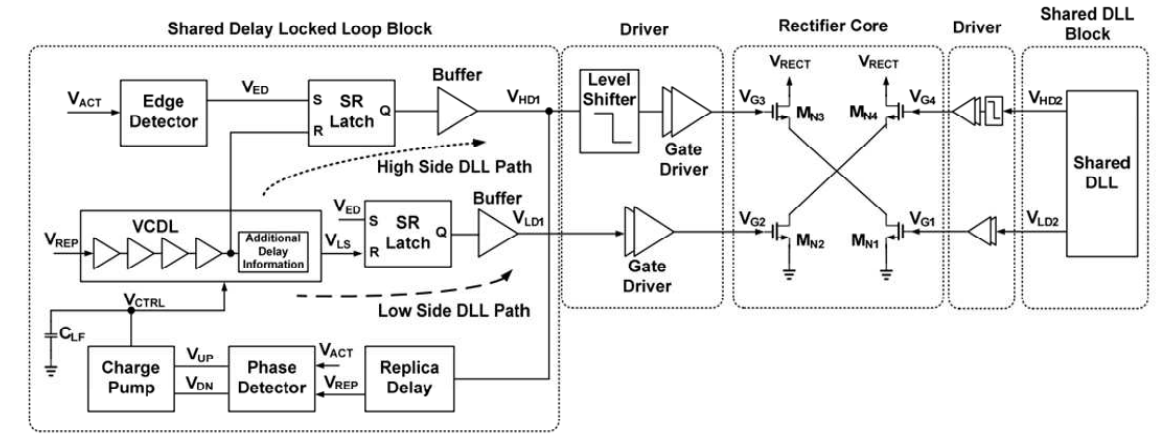

Figure 2.29: The schematic of a high-efficiency active rectifier with DLL in [41].

rent may bring serious problems. The proposed active rectifier uses the DLL to compensate for the delay caused by the gate driver delay and other internal circuits. Moreover, to accelerate the shutting-off process, a negative impedance circuit was utilized to accelerate the circuit. When the magnitude of the AC input voltage is $8.95 \mathrm{~V}$ of $6.78 \mathrm{MHz}$, the maximum efficiency of the proposed active rectifier is $91.5 \%$.

Based on the references quoted in the previous section, it is apparent that the active rectifier with cross-couple structure with fast comparator design had drawn the attention of researchers ever since the $13.56 \mathrm{MHz}$ RFID energy harvesting implementation. However, it is challenging to transfer such technology to the megahertz WPT for high power application since the extra power leads to larger transistor sizes. The increased sizes further incur larger delay of the comparator causes reverse current to happen, which deteriorate the efficiency of the rectifier. Ref [3] indicates that the delay time control would be suppressed for megahertz WPT application. However, the DLL would be slow when a load transition happens and the replica unit in [41] also caused area overhead in the design. In Section 4 of Chapter 4, the author proposed a rectifier with adaptive time delay control for the $6.78 \mathrm{MHz}$ WPT with $91.8 \%$ peak efficiency and $15 \mathrm{~W}$ output power. Detailed analysis and design description would be given in Chapter 4. A short summary is given at Table 2.1, the advantages and problems of each structure are listed in this table as well. 
Table 2.1: A summary about the rectifier

\begin{tabular}{|c|c|c|c|c|c|c|c|}
\hline Structure & {$[49]$} & {$[48]$} & {$[42]$} & {$[43]$} & {$[44]$} & {$[40]$} & {$[41]$} \\
\hline $\begin{array}{c}\text { Active } \\
\text { with } \\
\text { RCC }\end{array}$ & $\begin{array}{c}\text { Fast } \\
\text { Comp. }\end{array}$ & $\begin{array}{c}\text { Dual } \\
\text { Mode } \\
\text { Rect. }\end{array}$ & $\begin{array}{c}3 \mathrm{R} \\
\text { Rect. }\end{array}$ & $\begin{array}{c}\text { Dual } \\
\text { Mode } \\
\text { Rect. }\end{array}$ & $\begin{array}{c}\text { Half } \\
\text { Active } \\
\text { Rect. }\end{array}$ & $\begin{array}{c}\text { DLL } \\
\text { control } \\
\text { Recti. }\end{array}$ \\
\hline$V_{\text {out }}(\mathrm{V})$ & 3.5 & 1 & 3.1 & 5 & 3.6 & 5 & 8.6 \\
\hline $\begin{array}{c}P_{\text {out }} \text { ax } \\
(\mathrm{mW})\end{array}$ & 6.8 & 5 & 6 & 6000 & 102 & 6000 & 6000 \\
\hline $\begin{array}{c}\text { Resonant } \\
\text { f. }(\mathrm{MHz})\end{array}$ & 13.56 & 1.5 & 13.56 & 6.78 & 13.56 & 6.78 & 6.78 \\
\hline $\begin{array}{c}\text { Rectifier } \\
\text { Peak eff. }\end{array}$ & $87 \%$ & $87.5 \%$ & $77 \%$ & $86 \%$ & $92.6 \%$ & $89.2 \%$ & $91.5 \%$ \\
\hline Pros. & $\begin{array}{c}\text { Almost } \\
\text { zero } \\
\text { reverse } \\
\text { current }\end{array}$ & $\begin{array}{c}\text { Zero } \\
\text { reverse } \\
\text { current }\end{array}$ & $\begin{array}{c}\text { Zero } \\
\text { reverse } \\
\text { current }\end{array}$ & $\begin{array}{c}\text { Zero } \\
\text { reverse } \\
\text { current }\end{array}$ & $\begin{array}{c}\text { Almost } \\
\text { zero } \\
\text { reverse } \\
\text { current }\end{array}$ & $\begin{array}{c}\text { Zero } \\
\text { reverse } \\
\text { current }\end{array}$ & $\begin{array}{c}\text { Zero } \\
\text { reverse } \\
\text { current }\end{array}$ \\
\hline $\begin{array}{c}\text { Problems } \\
\text { for high } \\
\text { power } \\
\text { WPT }\end{array}$ & $\begin{array}{c}\text { Reverse } \\
\text { current } \\
\text { due to } \\
\text { high } \\
\text { power }\end{array}$ & $\begin{array}{c}\text { Reverse } \\
\text { current } \\
\text { due to } \\
\text { high } \\
\text { power }\end{array}$ & Diode & Diode & $\begin{array}{c}\text { Reverse } \\
\text { loss } \\
\text { loss } \\
\text { due to } \\
\text { high } \\
\text { power }\end{array}$ & Diode & $\begin{array}{c}\text { Extra } \\
\text { loss } \\
\text { area } \\
\text { and } \\
\text { Low } \\
\text { Speed }\end{array}$ \\
\hline
\end{tabular}

\subsection{Frequency Splitting}

Frequency splitting is a widely known phenomena in resonant wireless power transfer system. The concept of frequency splitting is from a mechanics model of two pendulums connected by a spring, which can be analogous to two LCR resonator connected by magnetic coupling [50]. In this single oscillating mechanic example, the two pendulums can have the largest oscillate amplitude in two frequencies, one of higher and one of lower frequency, which will be called as peak upper and lower peak frequency in the rest of this thesis. More importantly, the frequency separation of the peak frequencies is dependent on the coupling of the spring. As this coupling decreases, the frequency separation also decreases until the two peak frequencies converge to the resonate frequency of the system. This indicates that a coupling system can have peak output amplitude in different frequencies, which is a function of coupling. When it comes to WPT system, 
coupling is dependent on the distance between the transmitting and receiving coils.

[25] gave a detailed analysis on frequency splitting of $\mathrm{MHz}$ resonant WPT system based on a four-coils model shown in Figure 2.6. [25] takes the scattering parameters (S21), which is the forward voltage gain of the system, as the indicator of the figure of merit (FOM) of the system and call S21 as efficiency. According to both simulation and experimental experiment, shown in Figure 2.30, the critical coupling is defined as coupling between coil 2 and coil 3 when the system has the maximum $\mathrm{S} 21$, expressed as:

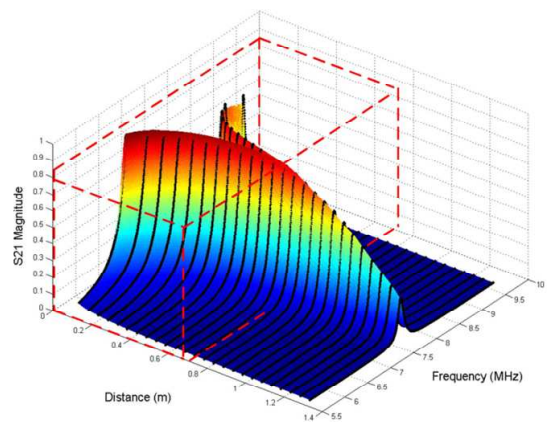

(a)

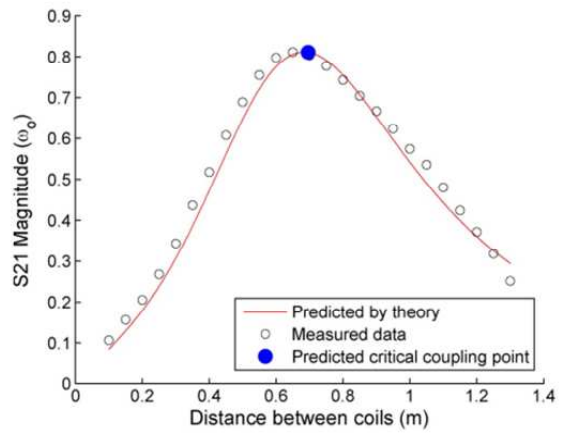

(b)

Figure 2.30: The experiment results of critical coupling of 5-10 MHz WPT in [25](a) Comparison of (black dots) experimental data to the calculation results of the system; (b)Measured and Predicted critical coupling.

$$
k_{\text {critical }}=\frac{1}{Q_{\text {coil }}}+\left(k_{\text {lc }}\right)^{2} Q_{\text {loop }} .
$$

in which it is assumed that the coupling between coill and coil2, coil3 and coil4 is fixed, defined as $k_{l c}$.

Although this paper presents a deep understanding of the underlying principles of coupled WPT system with a simple circuit model, a derivation of the optimum of forward voltage gain based on the system transfer function and a detailed explanation of frequency splitting, this paper takes S21 as efficiency incorrectly. The definition of the efficiency should be the ratio of the output power consumed on the load and the transmitted power from the AC source. Based on 
the conclusion of [25], [51] [52] further discussed the efficiency of a two-coil wireless power transfer system, which is expressed as:

$$
\eta=\frac{T_{Q}^{2}}{\left(1+S_{M}\right)\left(1+L_{M}\right)+T_{Q}^{2}+\frac{1+S_{M}}{1+L_{M}} F_{D}^{2}} \frac{L_{M}}{1+L_{M}} .
$$

where

$$
\begin{aligned}
& S_{M}=\frac{R_{S}}{R_{0}} \quad L_{M}=\frac{R_{L}}{R_{0}} . \\
& T_{Q}=\frac{\omega M_{12}}{R_{0}} \quad T_{Q}=\frac{1}{R_{0}}\left(\omega L_{0}-\frac{1}{\omega C_{0}}\right) .
\end{aligned}
$$

The optimal $L_{M}$ is calculated by taking $\partial \eta / \partial L_{M}$, giving that:

$$
L_{o p t}=\sqrt{\frac{1+S_{M}+T_{Q}^{2}}{1+S_{M}}} .
$$

Although these papers derive the condition where the system can achieve the maximum efficiency, the authors failed to considered the output power in such condition. Actually, the output power at such condition would be very low when the resonant frequency is several megahertz. The discussion of the trade-off of the output power and efficiency would be expanded in the chapter 5 in this thesis.

\subsection{Power Amplifier}

\subsubsection{Class D Amplifier}

Class D DC-AC RF resonant power amplifier was firstly reported by Baxandall in 1959 [53], and have been developed in different sorts of applications to convert DC power to AC power. A typical Class D half-bridge amplifier is shown in Figure2.31.

It is composed of two active switches $S_{1}$ and $S_{2}$ and a load resistor with a LC resonator. $S_{1}$ and $S_{2}$ are turned on and off alternately with a $50 \%$ duty cycle or a little less, so the output voltage is switched from $V_{I}$ and ground, realizing a DC to AC conversion. 


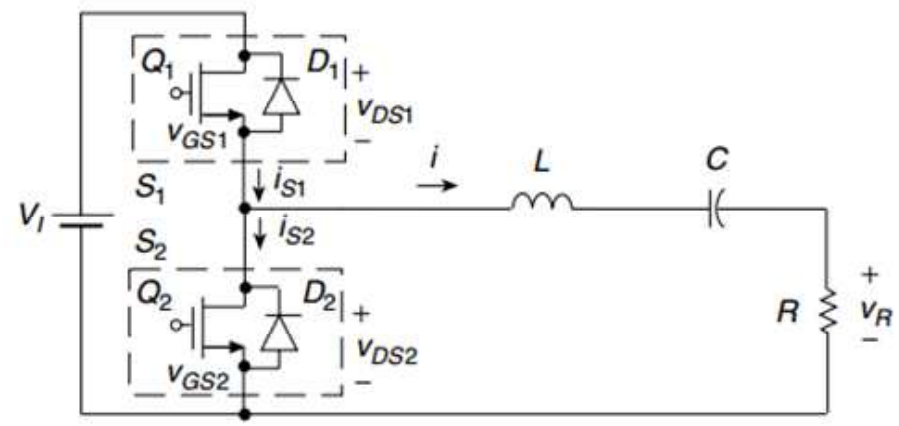

(a)

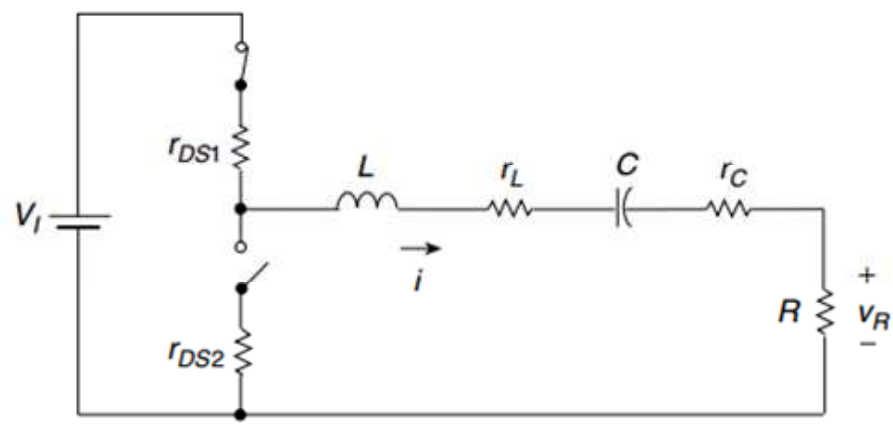

(b)

Figure 2.31: A typical Class D half-bridge amplifier:(a) Schematic.(b)Equivalent circuits.
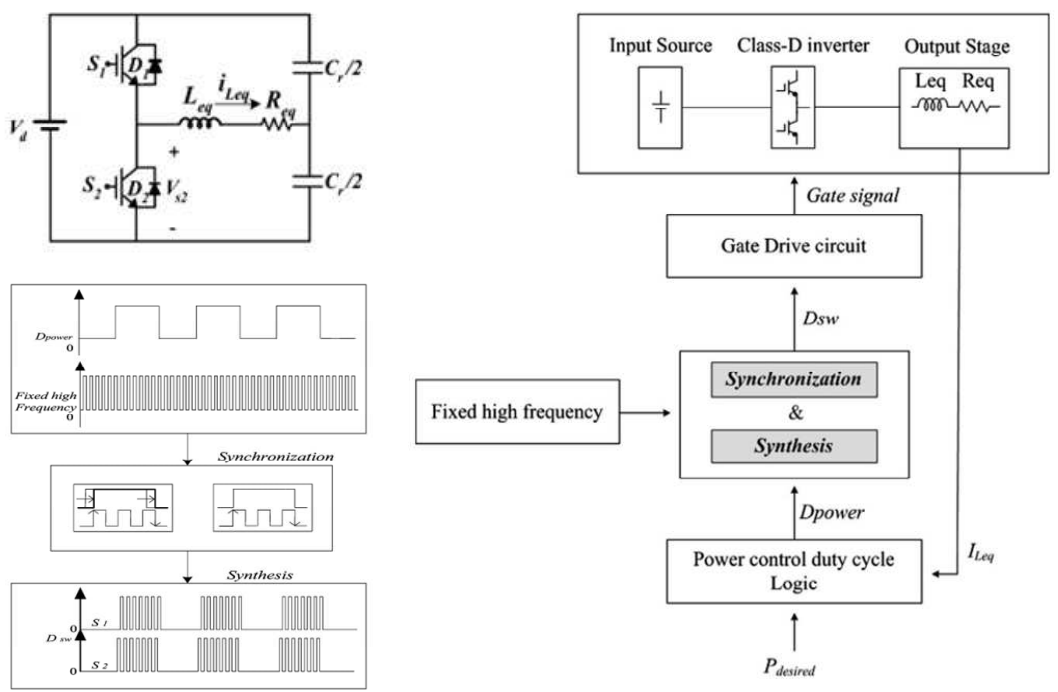

Figure 2.32: A Class D amplifier with power control in [54].

Reference [54] proposes a variable duty cycle power control algorithm for a fixed frequency class D amplifier for induction heating applications. The schematic of the circuit and control flow is shown in Figure 2.32. The difference between the load current $i_{L e q}$ and the desired power $P_{\text {desired }}$ determines $D_{\text {power }}$. After synthesis with a fixed high frequency to reduce spikes in low frequency, the signal 
triggers the gate drive to control the output power of the amplifier.

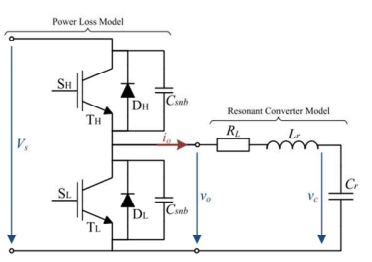

(a)

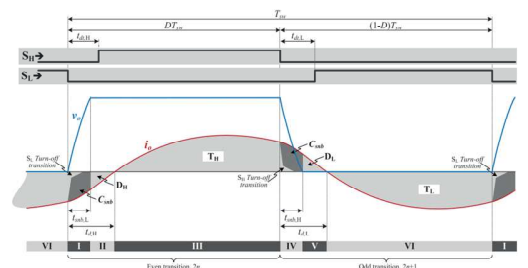

(b)

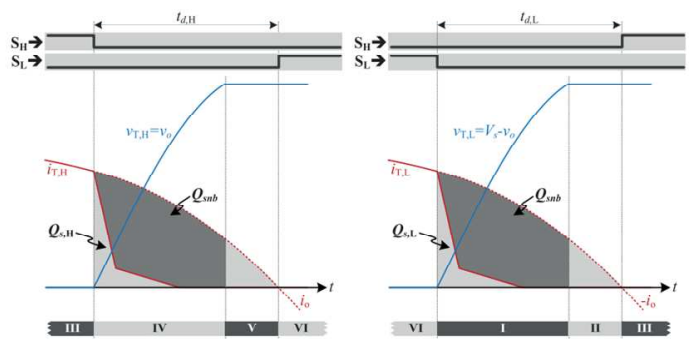

(c)

Figure 2.33: The analytical model in [55].

To improve the efficiency of the Class D amplifier, [55] presents an analytical model. In this reference, the circuit transitions during different working situations are analysed in details, as illustrated in Figure 2.33(b) and (c). After the analysis, detailed equations of the current and voltage of each transition are listed in this paper. This model accelerates the design procedure of a Class D amplifier. In addition, this reference also developed adaptive control emulation, which helps to improve the performance of the amplifier.

Reference [56] gives analytical expressions of a Class D amplifier in steady state for the ZVS condition in any duty cycle and proves them with a practical circuit. The output power capability, voltage and current waveforms, switch voltage, and current stress are shown in this paper. The main contribution of this reference is that the analysis is based on a variable compensation capacitor, which can be seen in Figure 2.34.

\subsubsection{Class E Amplifier}

Class E amplifiers are suitable for high-frequency DC-AC power conversion because they can easily achieve either ZVS or ZCS. A schematic of a basic ZVS Class E DC-AC converter is shown in Figure 2.35. 

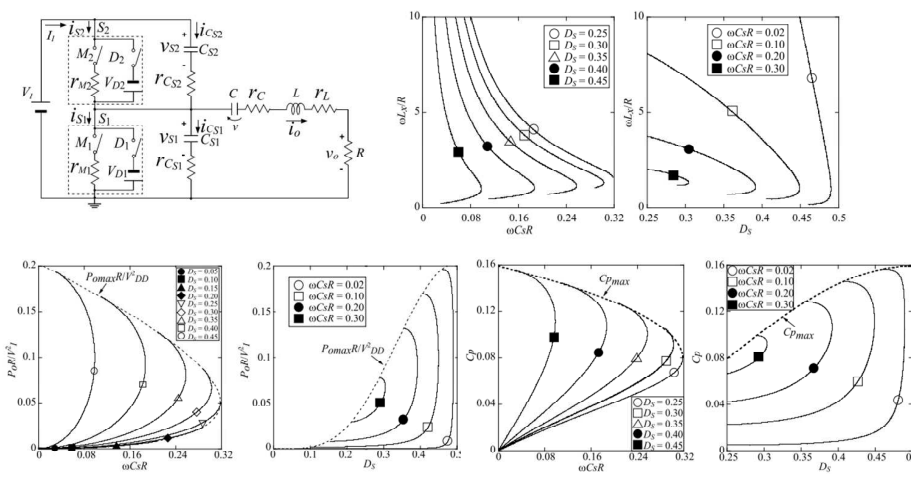

Figure 2.34: Circuits and Waveforms in [56].

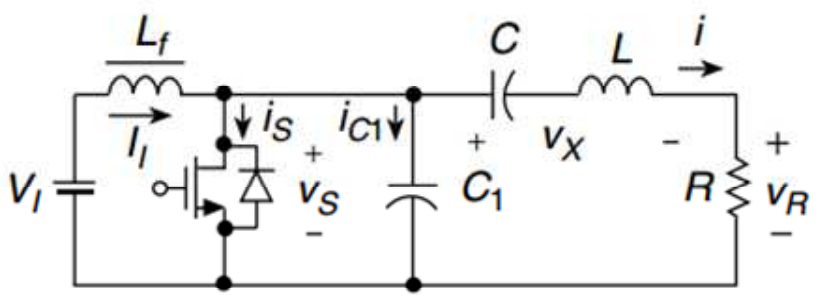

(a)

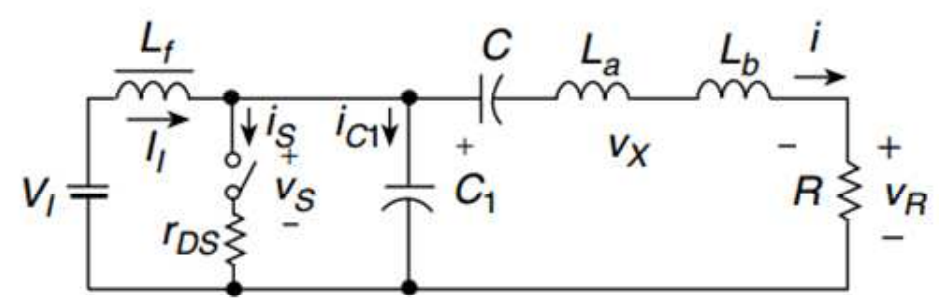

(b)

Figure 2.35: A typical Class E amplifier:(a) Schematic.(b)Equivalent circuits.

The basic design idea is that the current and voltage of the transistor are nonoverlapping during the operation, so the switching loss of the converter should theoretically be zero, leading to a very high efficiency. The amplifier comprises a power transistor, an $\mathrm{LC}$ resonator, a shunt capacitor $C_{1}$, a choke inductor $L_{f}$, and a load resistor R. Usually the choke inductor should be sufficient large so that it can be treated as a constant current source in the circuit. When the switch is off, the DC power source will charge the LC resonator and the load; when the switch is on, the energy stored in the LC resonator releases the power to the $C_{1}$, and since the resonant frequency is different between the LC resonator and the $\mathrm{L}, \mathrm{C}, C_{1}$ series network, the parameters of the circuit should be designed to make sure that the voltage of $C_{1}$ will be exactly zero at the end of the switch-off mode, 
resulting in a ZVS action.
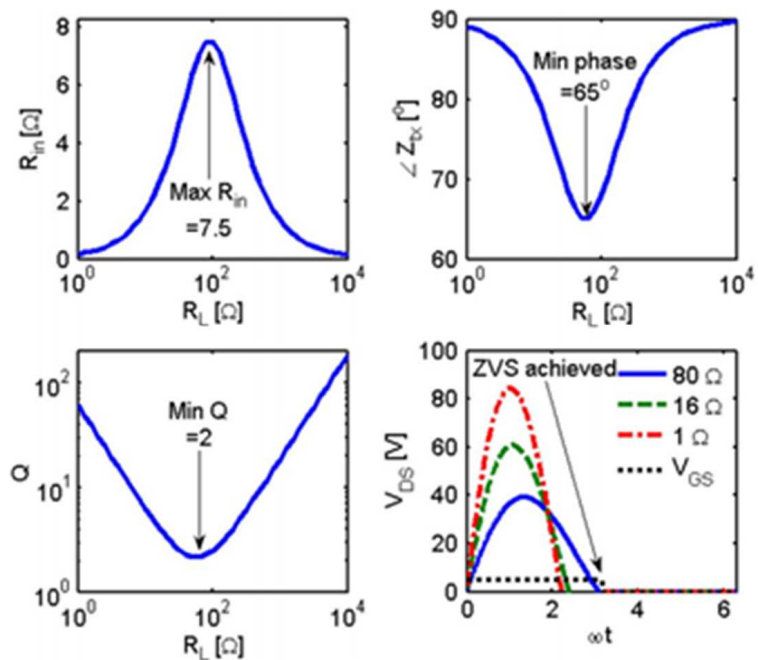

Figure 2.36: Analysis of Class E amplifier at different loads and frequencie in [57].

Reference [57] presents an example of a Class E amplifier application for a WPT system. This reference analyses the optimized conditions for a Class E amplifier at different loads and frequencies, as shown in Figure 2.36. Equations for the optimization have been derived for both transmitter and receiver. An experimental platform with an output power of $3.7 \mathrm{~W}$ and efficiency of $66 \%$ has been established to prove the design.

Reference [58] applied the Class E technique in both the transmitter and the receiver. The simulation results showed that the circuit could achieve ZVS/ZDS conditions for both sides with an output power of $5 \mathrm{~W}$ and an overall efficiency of 65.9\%. In this reference, reflected load theory (RLT) has been used to combine the receiver with the transmitter when analysing the optimized conditions for the Class E amplifier, while combining the transmitter with the receiver when analysing the optimized condition for the Class E rectifier. It shows that for the amplifier, the optimized condition relies on the equivalent reflected impedance as shown in Figure 2.37(c), while for the receiver, it depends on the equivalent impedance of the rectifier and the load.

Reference [59] extended the analysis of a CLASS Class E amplifier not only to ZVS, ZDVS, ZCS and ZDCS conditions, but also to an application without a 


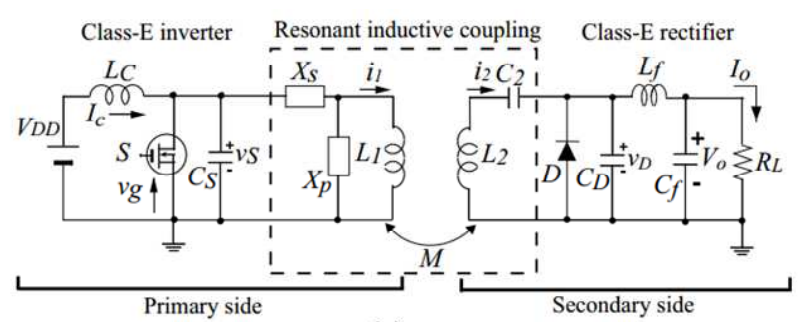

(a)

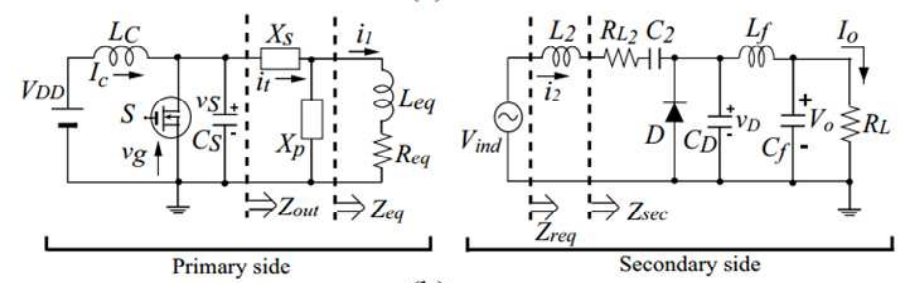

(b)

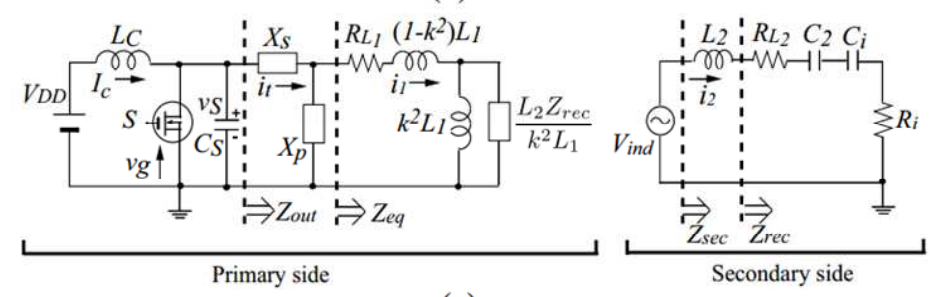

(c)

Figure 2.37: Equivalent circuits model Class E- Class E WPT system in [58].

tedious tuning process. The main contribution of this reference is that it takes the coil turns as a main parameter of the whole design procedure. Meanwhile, the Q value of the transmitter has been used to analyse the total harmonic distortion (THD) and the output power of the system. A detailed example is shown in Figure2.38. It can be seen that the high- frequency harmonics need to be considered as the $Q_{1}$ decreases. The resonator consisting of the coil and capacitor works as a filter, whose the performance of which deteriorates as the Q decreases. The validity of the analysis has been confirmed from the simulation and experimental results.
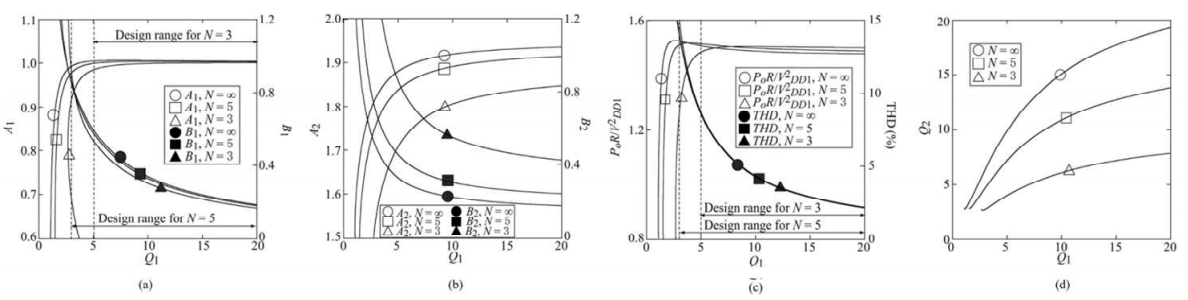

Figure 2.38: Design curves with the parameter $Q_{1}$ of [59]. 


\subsection{Summary}

In summary, the WPT technology has a lot of research directions, which include:

- WPT topologies design. In this field, the research mainly focuses on the maximum efficiency design or maximum output power design.

- High performance circuits design. In this field, for the transmitter design, the research mainly focuses on the ZVS/ZCS conditions design of the power amplifier; for the receiver design, high-efficiency rectifier and DC-DC design are important in the WPT system.

- Smart control design for WPT. In this field, the main objects of control include the voltage, amount and direction of power, and phase angle between the voltage and current.

In the next chapters, a single-stage AC-DC regulator for $125-\mathrm{kHz}$ WPT, a fullactive rectifier for 6.78-MHz WPT and a frequency/phase control design will be presented. 


\section{Chapter 3}

\section{Design of A Single-Stage AC-DC \\ Voltage Regulator for $125 \mathrm{kHz}$}

\section{Wireless Power Transmission}

\subsection{Introduction}

A basic WPT system consists of a transmitter and a receiver, as shown in Figure 3.1.

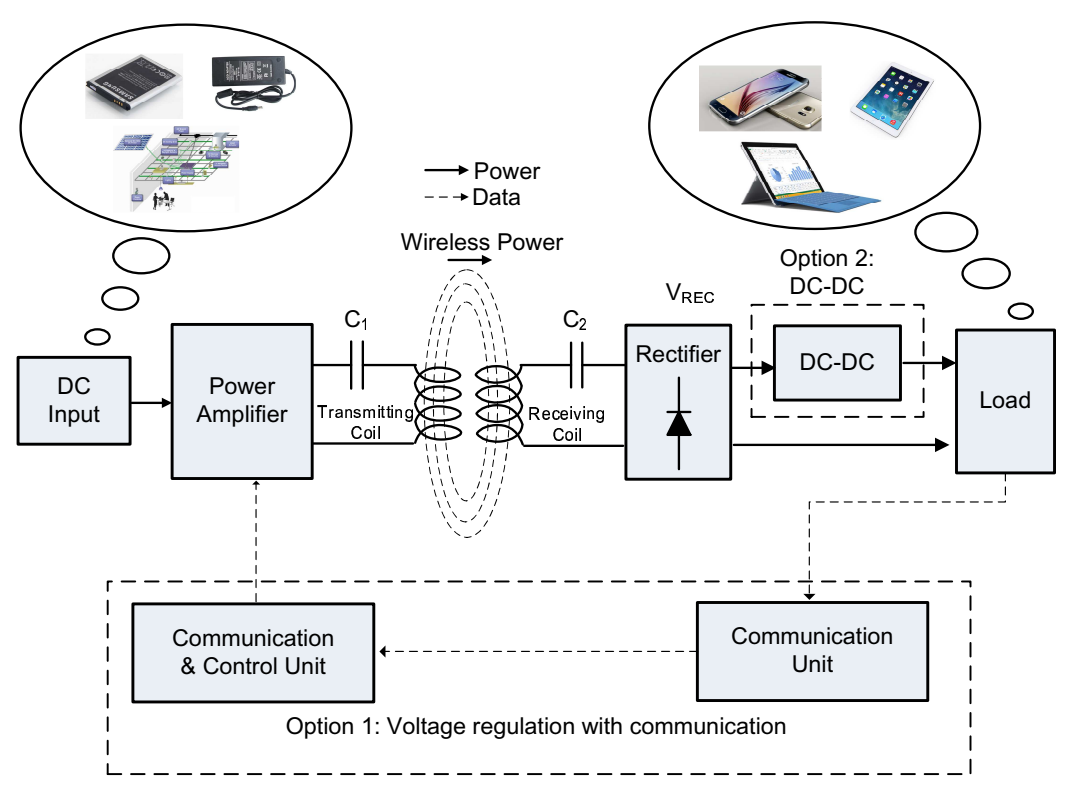

Figure 3.1: Wireless power transfer (WPT) system for consumer devices.

In the transmitter, the power amplifier (PA) transforms input DC voltage to a 
high frequency AC voltage that drives the transmitting coil to generate AC magnetic fluxes. In the receiver, the coupled AC voltage is rectified into DC voltage $\left(V_{R E C}\right)$ by a rectifier. In real-life WPT applications, the coupling coefficient of the two coils varies with the distance and the alignment between them. Moreover, the output current changes with time depending on the capacity of the battery. Both of these factors cause the $V_{R E C}$ to be unstable. Since most WPT standards for consumer electronic devices require the charger to be able to provide a constant voltage, like Qi standard [32], output voltage regulation is a key function of the system. The regulation can be achieved at either the transmitter [34] or the receiver [42]. For the former, wireless data communication blocks are required in the system, which essentially increase the design complexity and power loss. For the latter, the conventional approach uses a two-stage regulator consisting of a rectifier and a step-down converter, which can either be a low dropout regulator (LDO) with low efficiency at high voltage drop or a DC-DC converter with an inductor, but it is nonetheless difficult to be incorporated into an integrated circuit (IC). The resonant regulating rectifier (3R) structure was reported in [43] to address the aforementioned challenges; however the passive rectifier reduces the efficiency of the receiver.

In this chapter, a novel structure of AC-DC voltage regulator is proposed. The whole system structure is shown in Figure 3.2. This regulator consists of four power MOSFETs and a controller. The details of the circuit will be described in the following sub-sessions. As opposed to the existing systems mentioned earlier, this method does not need any wireless communication blocks and a step-down DC-DC converter. The regulator with a maximum output power of $15 \mathrm{~W}$ is able to provide over $90 \%$ efficiency over a large output power range.

The rest of the chapter is organized as follows. A detailed analysis of the feasibility and advantages of the design is presented in Section 3.2. In Section 3.3, the operating principle of the single-stage regulator with PWM control is described and the relationship between the output voltage and switching duty 


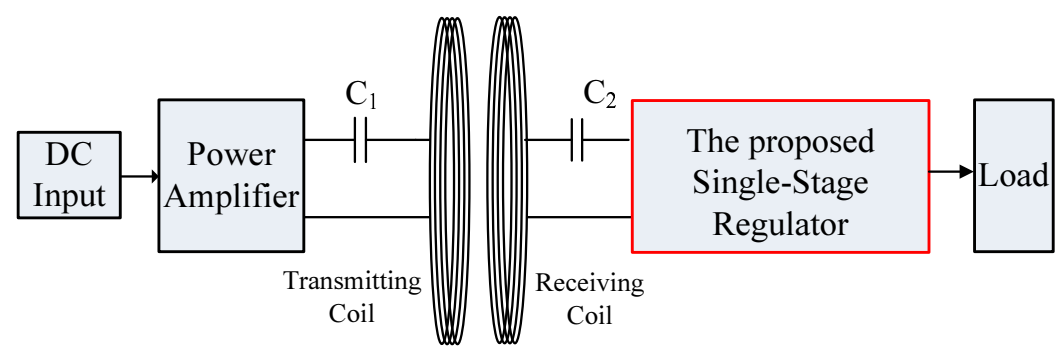

Figure 3.2: Proposed WPT system with proposed single-stage regulator.

ratio is derived. In Section 3.4, several important circuits and related techniques are presented and analyzed. Measurement results are presented and discussed in Section 3.5, followed by the concluding remarks in Section 3.6.

\subsection{Feasibility and Performance Analysis of the Novel Regu- lator}

As discussed before, voltage regulation at the transmitter incurs additional silicon cost as a result of complicated digital communication circuit blocks. Furthermore, the response of the regulation is not sufficiently fast as it is limited by the low data transfer rate of wireless communication technology. Since charging the consumer portable device requires rapid response, the regulation has to be included in the receiver, whereby a step-down DC-DC switch mode converter with a bulk inductor is to be used to regulate the voltage with high efficiency but the inductor is difficult to be integrated into a chip.

The idea of the proposed design originates from the basic operating principle of switched-mode power converter, which works on two basic configurations by switching storage elements (e.g. inductor) into different electrical states.

Take a step-down DC-DC converter for example, as shown in Figure 3.3. During the first configuration when $S_{1}$ is on while $S_{2}$ is off, the inductor $L_{1}$ builds up energy from the DC source. During the second configuration when when $S_{1}$ is off while $S_{2}$ is on, the DC input voltage source is disconnected, and the inductor release the energy to the load inorder to maintain the output voltage, which is 
usually called a freewheel mode [60], [61], [62], [63] and [64].

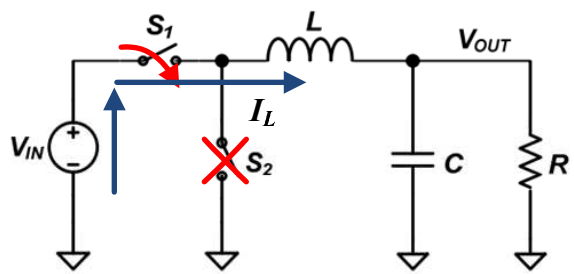

(a) Configuration 1

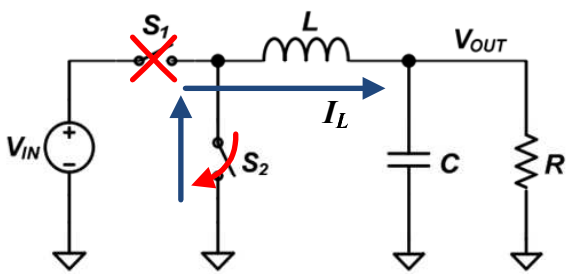

(b) Configuration 2

Figure 3.3: Operation of a traditional buck DC-DC Topologies.

In most cases, a PWM control algorithm is used to alternate the operation of the converter between the two configurations to achieve an optimal and accurate voltage regulation [65], [66], [67], [68] and [69]. The bulk inductor as a passive element plays a key role to store and provide the power for both configurations. There are in fact several other passive elements such as coils and capacitors, which can be utilized in WPT system.

In this work, we exploit the operating characteristics of the dual-configuration power converter as the seed idea for our proposed voltage regulated WPT system. During the charging configuration, the DC input transfers the power to the load and passive elements. During the non-charging configuration, no power is dissipated and the energy stored in the passive elements maintains the output voltage. It is crucial to design a high efficiency non-charging configuration since it determines the overall efficiency of the entire system.

\subsubsection{The Design of Non-Charging Configuration}

The non-charging configuration should have two important criteria: 1) The power from the transmitter should not reach the load; 2) The power loss should be minimal. One option of non-charging configuration, as shown in Figure 3.4 (a), is to disconnect the load in the receiver so as to cut off any power transmission from the transmitter, and the power loss in the receiver is close to zero. However, in this case, the current in the transmitter is substantially large since the coil and compensated capacitor $\left(C_{2}\right)$ form a short circuit loop at resonant frequency at the 
transmitter end, which in turn leads to substantially high current in the transmitter, potentially jeopardizing the operation of the transmitter. It is imperative to limit and control the transmitter current when the load is not being charged.
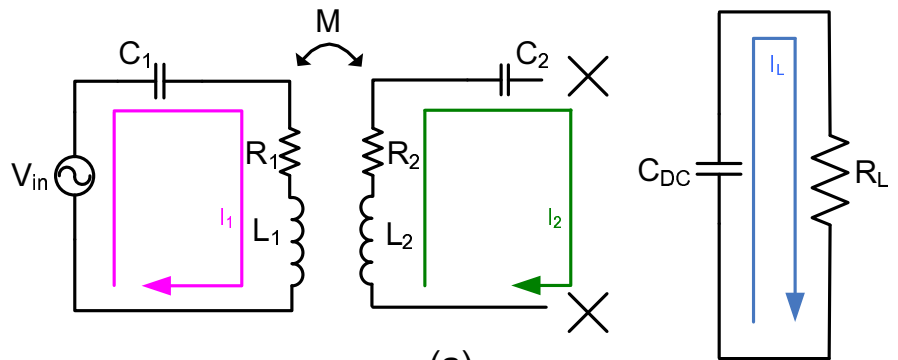

(a)

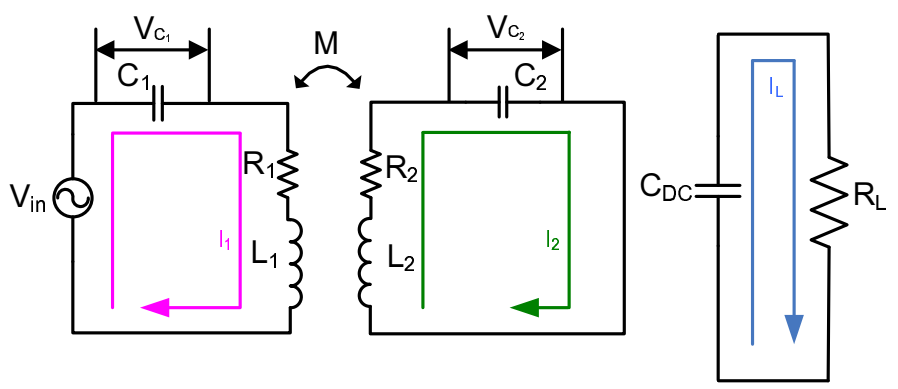

(b)

Figure 3.4: Two different non-configurations in WPT system (a) Disconnection mode; (b) Freewheeling loop mode.

To achieve low transmitter current for the non-charging configuration, a new topology is proposed and presented in Figure 3.4 (b). In this configuration, the receiving coil and its compensated capacitor form a short circuit loop with the load and the DC capacitor is disconnected. The voltage-current equations of this configuration is as follows:

$$
\left\{\begin{aligned}
V_{i n} & =I_{1}\left(R_{1}+j \omega L_{1}+\frac{1}{j \omega C_{1}}\right)+I_{2} j \omega M \\
0 & =I_{2}\left(R_{2}+j \omega L_{2}+\frac{1}{j \omega C_{2}}\right)+I_{1} j \omega M
\end{aligned}\right.
$$

In Equation 3.1, $V_{\text {in }}$ is the input equivalent $\mathrm{AC}$ voltage, $L_{1}, L_{2}, C_{1}$ and $C_{2}$, are the inductance of coils and capacitance of compensated capacitors respectively. $R_{1}$ and $R_{2}$ are the equivalent series resistance of coils. $I_{1}$ and $I_{2}$ are currents of the transmitter and receiver. In a resonant WPT system, ideally, the transmitter and receiver resonant frequencies are identical as given by 


$$
\omega=\frac{1}{2 \pi \sqrt{L_{1} C_{1}}}=\frac{1}{2 \pi \sqrt{L_{2} C_{2}}}
$$

From Equation 3.1 and Equation 3.2, we can get the value of $I_{1}$ and $I_{2}$ are given by

$$
\left\{\begin{array}{l}
I_{1}=\frac{V_{i n}}{R_{1}+\frac{\omega^{2} M^{2}}{R_{2}}} \approx \frac{V_{i n}}{\frac{\omega^{2} M^{2}}{R_{2}}} \\
I_{2}=\frac{I_{1} j \omega M}{R_{2}} \approx \frac{V_{i n}}{\omega^{2} M^{2}}
\end{array}\right.
$$

On contrary to the disconnection mode, it is apparent from Equation 3.3 that both $I_{1}$ and $I_{2}$ are finite values. In that case, the power loss in the noncharging configuration could be controlled by carefully design the value of $I_{1}$ and $I_{2}$. To further elaborate the difference between the non-connection mode and free-wheeling mode, the design equations and current of both transmitter and receiver are listed in Table. 3.1 And during the process of optimize the $I_{1}$ and $I_{2}$, it is important to keep the output voltage at the load adequately high for a 5 volt application. The definitions and criterions of the high efficiency operation area (HEOA) and the safe operation area (SOA) will be given and discussed in the next sections.

\begin{tabular}{|c|c|c|}
\hline Equations & Non-Connection & Free wheeling \\
\hline Transmitter Current & $I_{1}=V_{i n} \backslash R_{1}$ & $I_{2}=0$ \\
\hline Receiver Current & $I_{1}=\left(V_{i n}\right) R_{2} \backslash\left(R_{1} R_{2}+\omega^{2} M^{2}\right)$ & $I_{2}=\left(V_{\text {in }}\right) \omega M \backslash\left(R_{1} R_{2}+\omega^{2} M^{2}\right)$ \\
\hline
\end{tabular}

Table 3.1: Design equations of Non-Connection and Free wheeling Mode

\subsubsection{High Efficiency Operation Area Analysis}

For the charging configuration, a prior condition of having sufficient output voltage across the load when the output current is at its maximum is required. 
The voltage-current equation of charging configuration is as follows

$$
\left\{\begin{array}{l}
V_{\text {in }}=I_{1}\left(R_{1}+j \omega L_{1}+\frac{1}{j \omega C_{1}}\right)+I_{2} j \omega M \\
0=I_{2}\left(R_{2}+R_{L}+j \omega L_{2}+\frac{1}{j \omega C_{2}}\right)+I_{1} j \omega M
\end{array}\right.
$$

Since there is a limitation on the voltage level of the input for safety considerations, the voltage gain is required to be high enough for a $5 \mathrm{~V}$ application. Based on Equation (3.2) and (3.4), the voltage gain of the system during charging is derived as

$$
\text { Gain }=\frac{I_{2} R_{L}}{V_{\text {in }}}=\frac{\omega M R_{L}}{R_{1}\left(R_{2}+R_{L}\right)+\omega^{2} M^{2}}
$$

For the non-charging configuration, all power consumption should be considered as power loss, which is listed in Equation 3.6. This power loss should be an acceptably small fraction of the output power.

$$
P_{\text {Loss }} \approx \frac{V_{\text {in }}^{2}}{\left(R_{1}+\frac{\omega^{2} M^{2}}{R_{2}}\right)^{2}} R_{1}+\frac{V_{\text {in }}^{2}}{\left(\frac{\omega^{2} M^{2}}{R_{2}}\right)^{2}} R_{2}
$$

In the design of the non-charging circuit configuration, a sufficiently large voltage gain and a minimal power loss are two key requirements of the system. Based on the measurement data of the coil $\left(R_{1}, R_{2}\right.$ and $\left.\mathrm{M}\right)$, it can be considered that the term " $\omega^{2} M^{2}$ " of Equation (3.5) is the dominant factor. As a result, the voltage gain of WPT system is inversely proportional to the mutual inductance (M); while Equation (3.6) suggests that the power loss is inversely proportional to $\mathrm{M}$. The voltage gain and power loss trade-off makes the value of $\mathrm{M}$ a crucial design parameter.

For voltage gain, the recommended input DC voltage by Qi standard is 19 $\mathrm{V}$ and the output voltage is usually $5 \mathrm{~V}$ for most consumer electronic devices. In this regard, the voltage gain should not be less than 0.26. For power loss, a maximum of $1 \mathrm{~W}$, which is $5 \%$ of the output power, is set for the proposed design. And this is the definition of the high-efficiency operation area (HEOA). 
Table 3.2: Mutual Inductance from $4 \mathrm{~mm}$ to $8 \mathrm{~mm}$

\begin{tabular}{|c|c|}
\hline Distance $(\mathrm{mm})$ & $\mathrm{M}(\mu \mathrm{H})$ \\
\hline 4 & 8.36 \\
\hline 5 & 7.93 \\
\hline 6 & 7.58 \\
\hline 7 & 7.24 \\
\hline 8 & 6.91 \\
\hline
\end{tabular}

The experimental results of the charging coil show that the resistors of coils at $125 \mathrm{kHz}$ are around $0.06 \mathrm{ohms}$. In this design, a 5V/3A output is required, so the $R_{L}$ is set to 1.6 ohms. The Gain vs. $\mathrm{M}$ and Power Loss vs. $\mathrm{M}$ curves are plotted in Figure 3.5. In the range of $0 \mu \mathrm{H}-8.4 \mu \mathrm{H}$, the voltage gain is larger than 0.25 ; in the range of $6.8 \mu \mathrm{H}-12 \mu \mathrm{H}$ the power loss is less than $0.8 \mathrm{~W}$. So the HEOA of the WPT system is limited to within the red dotted rectangle, which is from $6.8 \mu \mathrm{H}$ to $8.4 \mu \mathrm{H}$.

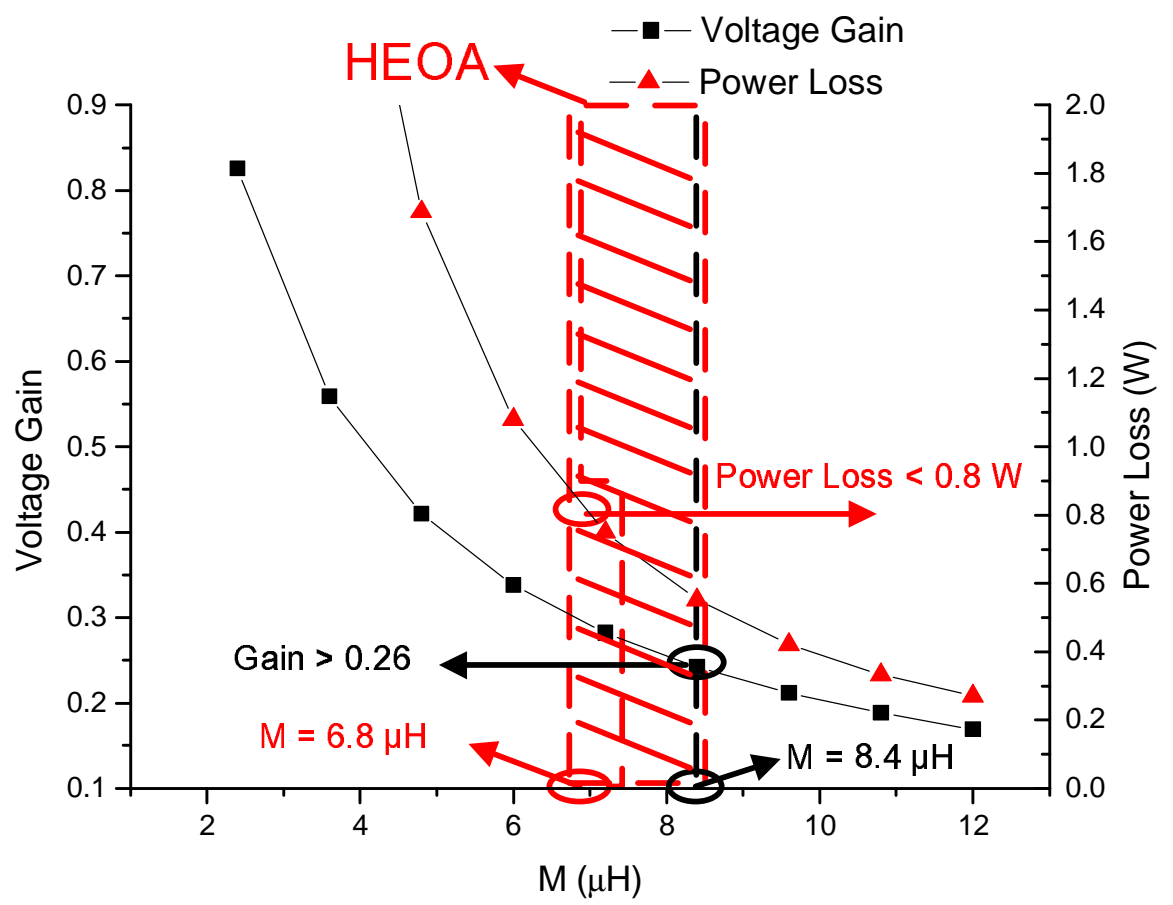

Figure 3.5: Voltage Gain vs. M and Power Loss vs. M.

For kilo Hertz WPT applications, the reasonable charging distance is around $5 \mathrm{~mm}$. The mutual inductance of this distance range is tested with a pair of coils of the same size and with a self-inductance of $11 \mu \mathrm{H}$. Table 3.2 provides the experimental results for the required mutual inductance for the distance range of 
$4 \mathrm{~mm}-8 \mathrm{~mm}$. It shows that in this range, the mutual inductance is located inside the HEOA, which corroborates the feasibility of this design.

\subsubsection{Safe Operation Area Analysis}

The two basic safety considerations in WPT system are currents through the coils, switches and voltages across the compensated capacitors [70], [71] and [72], the equations for the current in the coils are the same as Equation (3.3) and equations of the voltages cross the capacitors are given by

$$
\begin{aligned}
& V_{C 1}=I_{1} \frac{1}{j \omega C_{1}} \\
& V_{C 2}=I_{2} \frac{1}{j \omega C_{2}}
\end{aligned}
$$

Based on the values of self-inductance of the charging coils, the values of $V_{C 1}$ and $V_{C 2}$ can be calculated. The simulation results of $I_{1}, I_{2}, V_{C 1}$ and $V_{C 2}$ are shown in Figure 3.6. The current is in the range of $0 \mathrm{~A}$ to $5.5 \mathrm{~A}$, while the voltage is in the range of $0 \mathrm{~V}$ to $90 \mathrm{~V}$. This range is considered to be safe for both coils and capacitors.

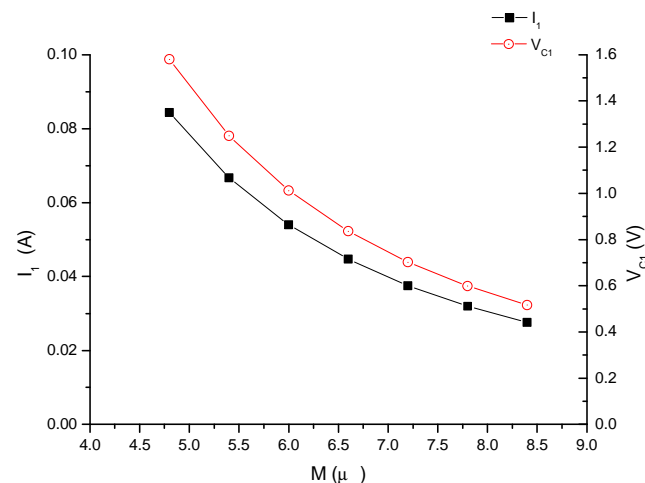

(a)

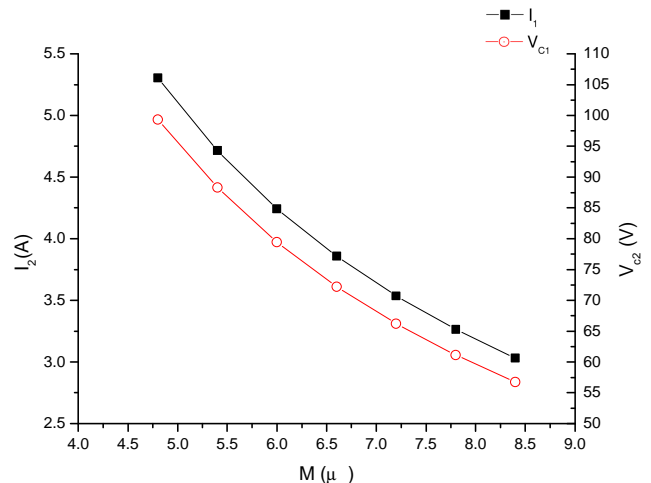

(b)

Figure 3.6: (a) $I_{1}$ and $V_{C 1}$ vs. $\mathrm{M}$ (b) $I_{2}$ and $V_{C 2}$ vs. $\mathrm{M}$. 


\subsection{Proposed Single-Stage Voltage Regulator for Wireless Power Transfer}

As mentioned in 3.2.1, if the receiver works in the configuration as depicted in Figure 3.4(b), it would provide a low power loss and safe non-charging configuration. In the proposed single-stage voltage regulator, four active switches are used to replace the diodes and a PWM controller is used to make these four switches to work between the charging and non-charging configuration to regulate the voltage. The detailed operation principle and the relationship between output voltage and the duty cycle of the charging configurations are analyzed with a phasor transformation [73], [74] and [75] in this section.

\subsubsection{Structure and Operation of the Proposed Single-Stage Voltage Regu- lator}

The basic structure of the regulator is shown in Figure 3.7 (a). The four active switches $\left(S_{1}, S_{2}, S_{3}\right.$ and $\left.S_{4}\right)$ are controlled by a PWM controller. When the controller gives a positive voltage $\left(V_{c t r l}\right)$, the four switches work in the charging configuration as an active rectifier, which is shown in Figure 3.7 (b). In this charging configuration, when the input $\mathrm{AC}$ voltage is in its positive half period, switches $S_{1}$ and $S_{4}$ are closed so the current flows through them to the load, while in the negative half period the current flows through $S_{2}$ and $S_{3}$. If the PWM controller gives a negative voltage, the regulator works in non-charging configuration as shown in Figure 3.7 (c), in which $S_{1}$ and $S_{4}$ are opened and $S_{2}$ and $S_{3}$ are closed. The closed $S_{2}$ and $S_{3}$ form a short circuit together with the receiver coil and the compensated capacitor, as discussed above. Unlike the work published in [43], which is a structure of a passive rectifier followed by a PWM controlled capacitor converter, it can be seen from Figure 3.7(a) that the circuits between the compensation capacitor and the load are only a power stage consisting of four active switches, which replaces the four diodes of a full-wave 
rectifier, and controlled by a PWM controller. The merger of four active switches and PWM controller can be considered as a single-stage AC-DC converter

The control period T equals 16 times the periods of the charging periods [76]. We define the duty of the duration of the charging configuration as D and the duration of non-charging as (1-D). The waveform of the current in the receiver coil is shown in Figure 3.7(d). It can be seen that in the charging configuration, the current $I_{2}$ slowly rises up to the charging current and becomes stable. In the non-charging configuration, $I_{2}$ slowly reduces to the value that can be calculated from (3) and becomes stable.

\subsubsection{Equivalent Model WPT System with Single-Stage Voltage Regulator}

In this section, the relationship between the duty-cycle of the charging configuration and the output voltage is analysed in detail. First, the WPT system is simplified to Figure 3.8 (a). The equivalent series resistance and capacitance of the coil are removed for the analysis. For simplicity, it is assumed that the switches in the power stage are ideally loss-less in Figure 3.8 (a). In the charging configuration, the switches works as an active full-wave rectifier so that the power directly goes to the load while in the non-charging configuration, the switches short the receiver, which means it is the same as Figure 3.7 (c). The equivalent circuit model of a mutual inductance is used to further simplify Figure 3.8 (a) to Figure 3.8(b). Since the current in the proposed WPT system is continuous, a phasor transformation method [73], which is useful to analyze the characteristics of a series resonant converter quantitatively, could be used to turn a rotational system into a stationary system. With this transformation, the inductors and capacitors in Figure 3.8 (b) are simplified to Figure 3.8 (c) [73]. And an equivalent $V_{R E G 1}$, $R_{D C 1}$, lossless switch and $C_{D C 1}$ are shown in Figure 3.8 (c) to replace the rectifiers. As discussed before, the control switching frequency is much lower than the resonant charging frequency of the WPT system. Therefore in Figure 3.8 (d), the equivalent inductors and capacitor could be removed since the system could 


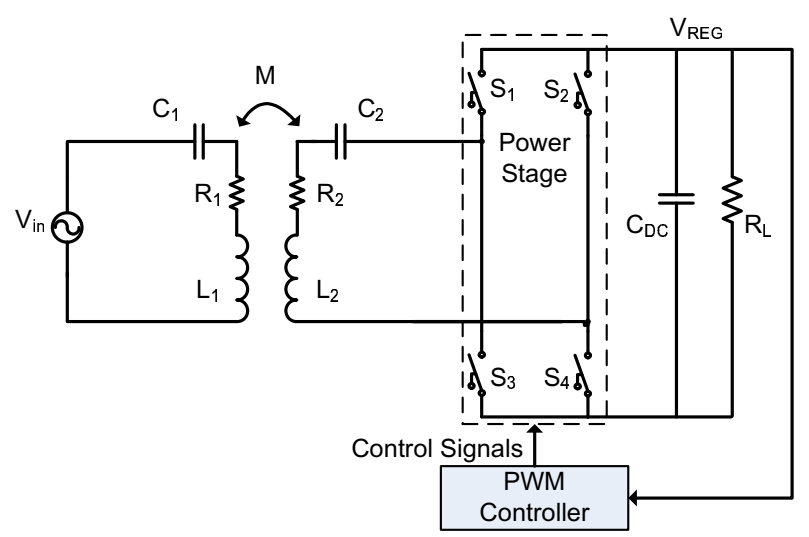

(a)

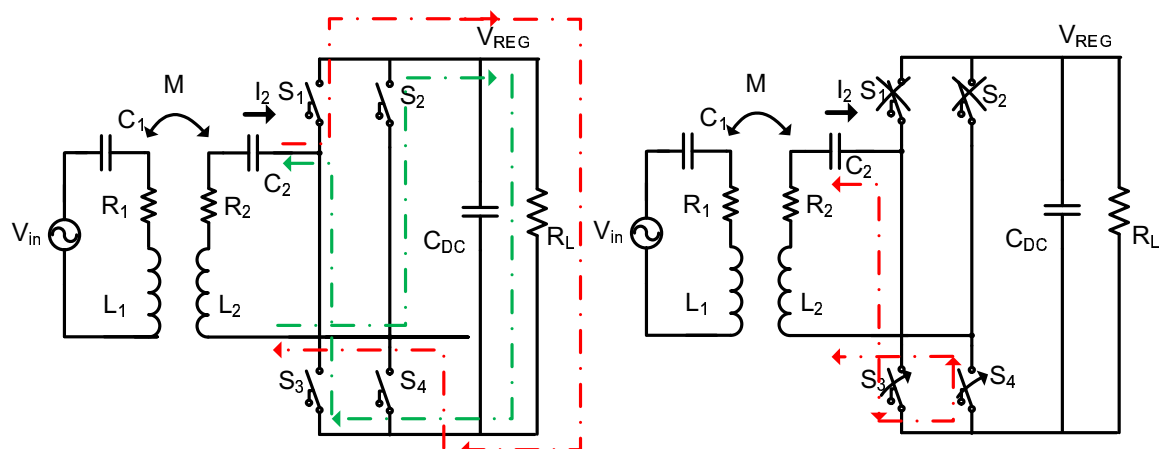

(b)

(c)

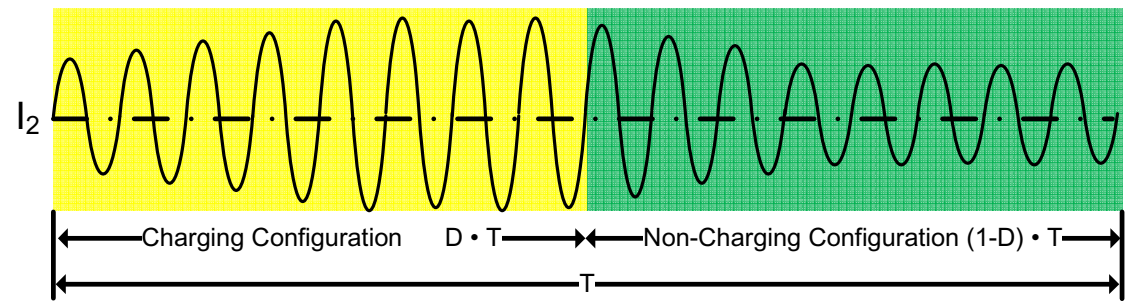

(d)

Figure 3.7: Operation principle of the proposed regulator (a) overall structure (b) charging configuration (c) non-charging configuration (d) current of the receiving coil.

be considered as a DC equivalent circuit. A Norton transformation is applied to transfer Figure 3.8(d) to Figure 3.8 (e). It could be concluded that after transformation, the model is simplified to a switched DC current to voltage converter in accordance with [73]. The details of the theory of the phasor transformation of $\mathrm{L}, \mathrm{C}$ and $\mathrm{R}$ are given in the appendix.

In the charging configuration, the switch is open so that the current $I_{e q}$ flows directly into $C_{D C 1}$ and $R_{L 1}$, and the charging current of the capacitor $C_{D C 1}$ is equal to $I_{e q}-I_{\text {out } 1}$. In the non-charging configuration, the $I_{e q}$ flows through the switch so 


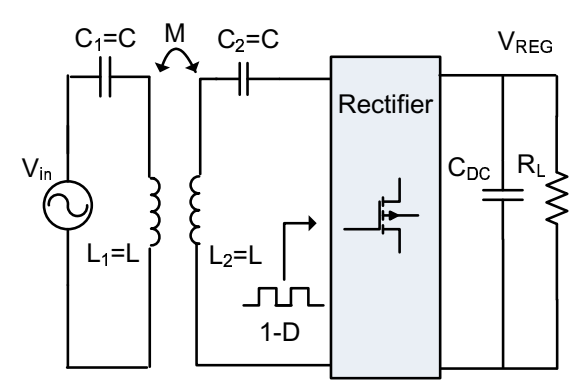

(a)

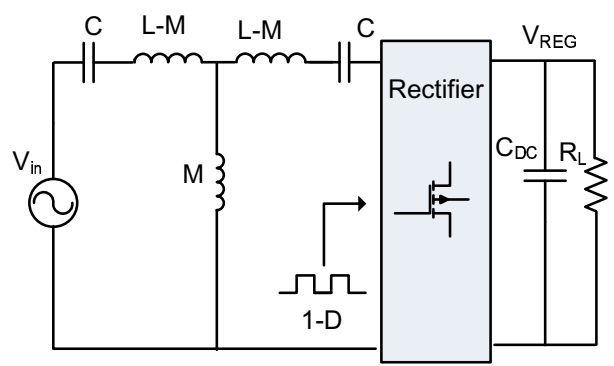

(b)

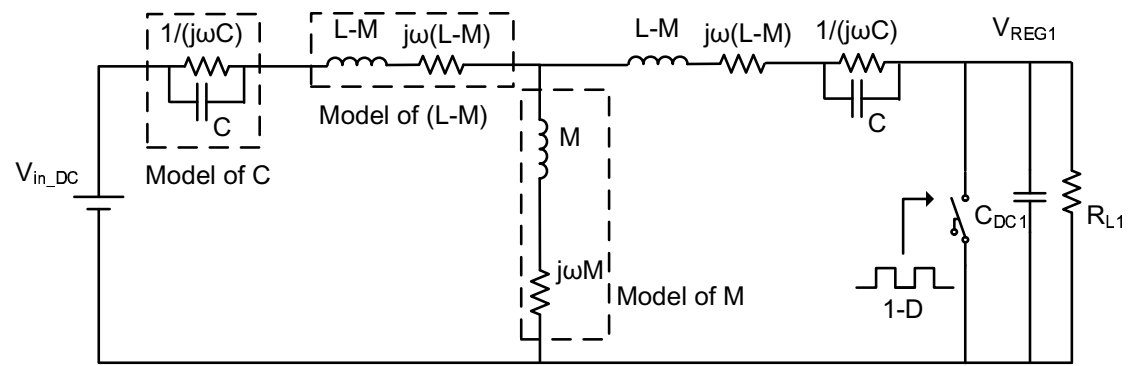

(c)

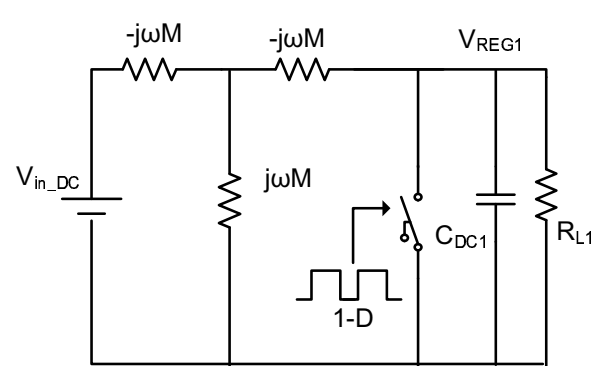

(d)

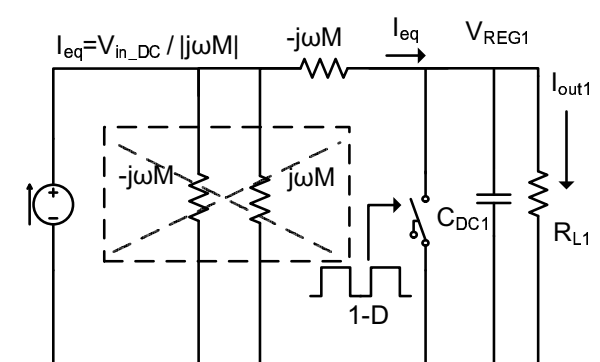

(e)

Figure 3.8: Model of the WPT system with the proposed single-stage voltage regulator.

that the discharge current of $C_{D C 1}$ equals $I_{\text {out } 1}$. The D- $V_{R E G 1}$ relationship can be derived as follows:

$$
\left(I_{\text {eq }}-I_{\text {out } 1}\right) \times D=I_{\text {out } 1} \times(1-D)
$$

Since $I_{\text {out } 1}$ equals $V_{R E G 1}$ over $R_{L 1}$, we have

$$
V_{R E G}=V_{R E G 1} \times I_{e q} \times R_{1} \times D \times G_{v}
$$

where $G_{v}$ is the voltage gain of a full-bridge rectifier,which equals $\pi^{2} / 8$ [77] [73].

The linearity of the $D-V_{R E G}$ is simulated in a Simulink model and shown in 
Figure 3.9, which proves the proposed model.

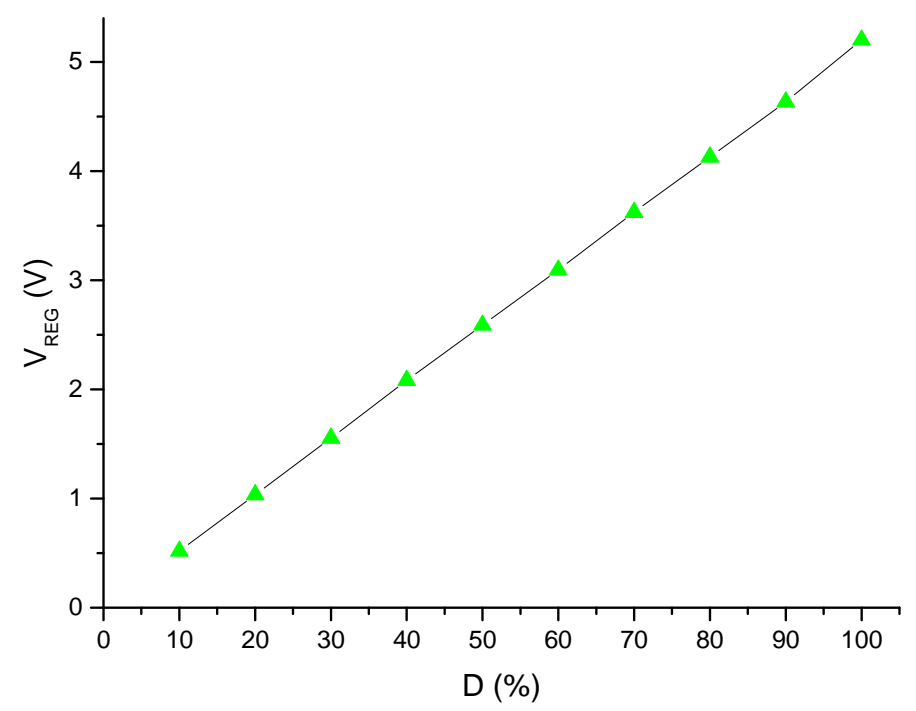

Figure 3.9: Simulation result of $V_{R E G}$ vs. D.

\subsection{Circuit Structure and Key Blocks}

The proposed voltage regulator with all peripheral circuit blocks is displayed in Figure 3.10. To meet the basic requirements of the WPT system, the regulator is designed to support a $15 \mathrm{~W}$ output at $5 \mathrm{~V}$ for fast charging of cellphones or tablets. The resonant frequency of the WPT system is set to $125 \mathrm{kHz}$ like many other Qi standard circuits. To control the regulator, the frequency of PWM signal is set to $7.8 \mathrm{kHz}$, which means the control period is 16 integral cycles per charging period.

Due to the limitation of the CMOS process, the power stage is not included in this chip design. Other sub-circuits like comparators, ramp generator, error amplifier and digital controllers are fabricated in a $0.35 \mu \mathrm{m}$ AMS technology, which is in red dash lines in Figure 3.10 (a). When the PWM signal is set to " 1 " by the feedback loop, the regulator works in the charging configuration, as depicted in Figure 3.10 (b), which comprises a crossed-connected rectifier [49], [47], [78], [79] and [80]. The operation of this circuit for the positive half period can be explained as follows: when $V_{H L}$ is zero, all four MOSFETs 


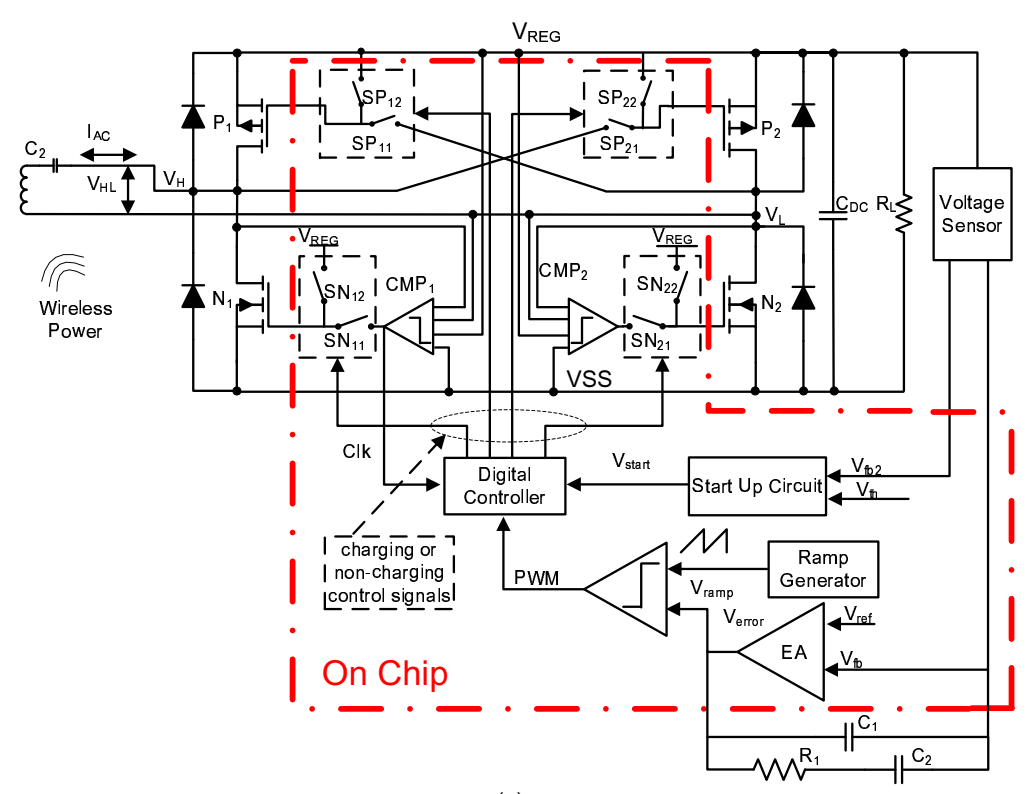

(a)

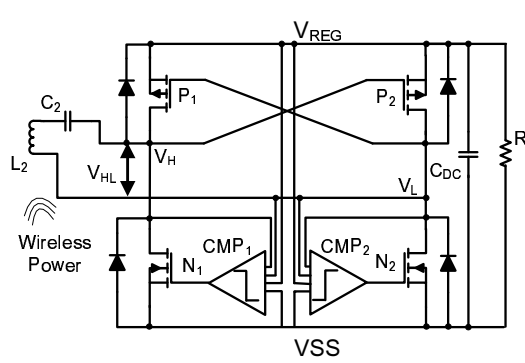

(b)

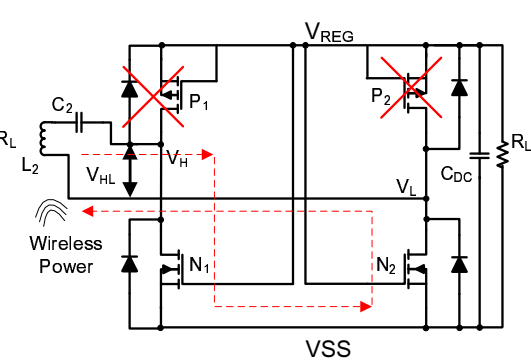

(c)

Figure 3.10: Circuit implementation of the proposed voltage regulator (a) Complete blocks of the regulator; (b) Circuits for charging configuration; (c) Circuits for non-charging configuration.

are turned off; subsequently, $V_{H L}$ increases gradually as the AC input voltage increases; when $\left|V_{R E G}-V_{L}\right|$ becomes larger than the threshold voltage of a PMOSFET $\left(\left|V_{t p}\right|\right), P_{2}$ gradually turns on, connecting $V_{H}$ to $V_{R E G}$; following that, the increasing AC voltage $V_{H L}$ continuously pushes $V_{L}$ until it drops below VSS. As a result, the $C M P_{2}$ turns on $N_{2}$ so that the charging current flows through the branch of $P_{2}$ and $N_{1}$ thereby charging towards $V_{R E G}$; after $V_{H L}$ reaches the peak, it begins to decrease so $V_{L}$ starts to rise; $C M P_{2}$ turns off the $N_{2}$ when $V_{L}$ is above VSS; $V_{L}$ keeps rising and $P_{1}$ is turned off when $\left|V_{R E G}-V_{L}\right|$ is smaller than $\left|V_{t p}\right|$. The operation of the circuit for the negative half period is similar to its positive counterpart.

When the PWM signal is set to "0" by the feedback loop, the regulator works in the non-charging configuration, as portrayed in Figure 3.10 (c). The two 
PMOSFETs are disabled and two NMOSFETs are turned on to form a freewheeling loop. In this case, a short circuit is formed in the receiver end so that $V_{R E G}$ gradually decreases. According to the RLT (reflected load theory) [13], [81], [82] and [83], a short circuit reflects large equivalent impedance to the transmitter, which dramatically decreases the current of the transmitter. The limited transmitter current brings a small induced voltage to the receiver, thereby limiting the current and the power loss of the regulator as was analysed in previous sections. The DC current to the load is provided by the smoothing capacitor $C_{D C}$. Therefore, it is inevitable for the $C_{D C}$ to be a large capacitor of $320 \mu \mathrm{F}$ in this design and it is the only component which cannot be integrated onto the chip (Note: Even though the power transistors are also not integrated in our current prototype, they can be readily integrated if $\mathrm{BCD}$ process is available). Nonetheless, the main feature of the proposed single-stage AC-DC voltage regulator is that it significantly reduces the design complexity of the entire WPT system and at the same time enhances the power efficiency by doing away with DC-DC converter or communication modules. The main building blocks are discussed in detail in the following subsections.

\subsubsection{Main Comparator Design}

The main comparator $\left(C M P_{1}\right.$ and $C M P_{2}$ in Figure 3.10 (a)) compares the voltage difference between the AC input voltage $\left(V_{H}\right.$ or $\left.V_{L}\right)$ and the DC output voltage $\left(V_{R E G}\right)$ to drive the NMOSFETs. As such, the comparator has to turn on/off the NMOSFETs with very little delay as $\left(V_{H}\right.$ or $\left.V_{L}\right)$ crosses $\left(V_{R E G}\right)$, or else the reverse current causes large extra power loss. Meanwhile, since the input dynamic range of the comparator has to cover the full voltage range of $V_{H^{-}} V_{L}$, the comparator also has to handle the low voltage. Since the common-gate comparator is a fast and low-power-loss structure [47], [84] and [85], it is used in this design and described in Figure 3.11 .

Take $C M P_{2}$ for example, when the $\mathrm{AC}$ voltage is larger than the $\mathrm{DC}$ voltage, 


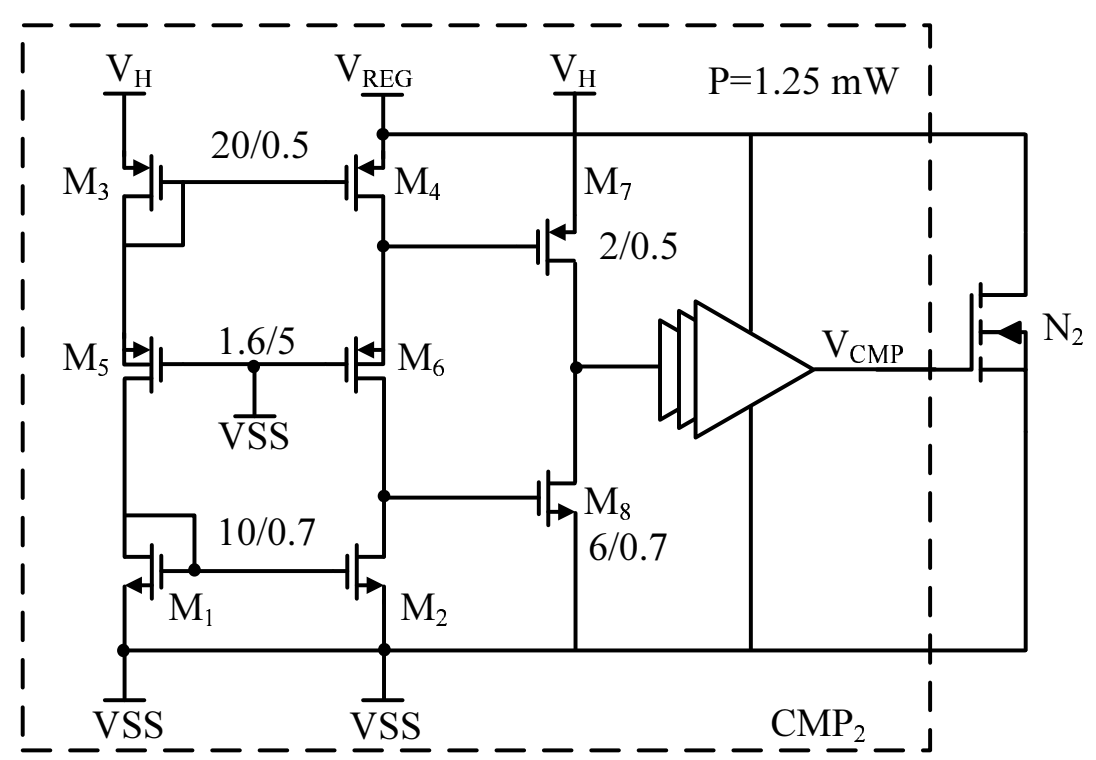

Figure 3.11: Schematic of Main comparator $\left(C M P_{2}\right)$.

which means $V_{H}$ goes beyond $V_{R E G}$, the gate of $M_{3}$ and $M_{1}$ increases to a certain level that turns off $M_{4}$ while turning on $M_{2}$. As a result, $M_{7}$ is turned on while $M_{8}$ is turned off. Additional drivers are added to drive the large input capacitance of the power MOSFETs $N_{2}$. When the $V_{H}$ goes below $V_{R E G}, M_{4}$ is turned on and $M_{2}$ is turned off, so the driver turns off $N_{2}$. To guarantee low reverse leakage current, the turn-off operation of power MOSFETs is the main concern. So in this design, the size of $M_{4}$ is a bit larger than $M_{3}$ to slightly strengthen the pull-up action , avoiding the reverse leakage current. The power consumption of the comparator is about $1.25 \mathrm{~mW}$.

The simulation results of the comparator and the AC input voltage are shown in Figure 3.12. From this figure, it can be seen that for the turn on action of the switch, the driving signal $\left(V_{C M P}\right)$ has a several nano seconds delay. Since, the reverse leakage does not happen during switch on action, such a delay does not decrease the efficiency. For the turn off action, the MOSFET can be turned off much faster, the leakage happens at the overlap area of the green line and red line, which is small. 

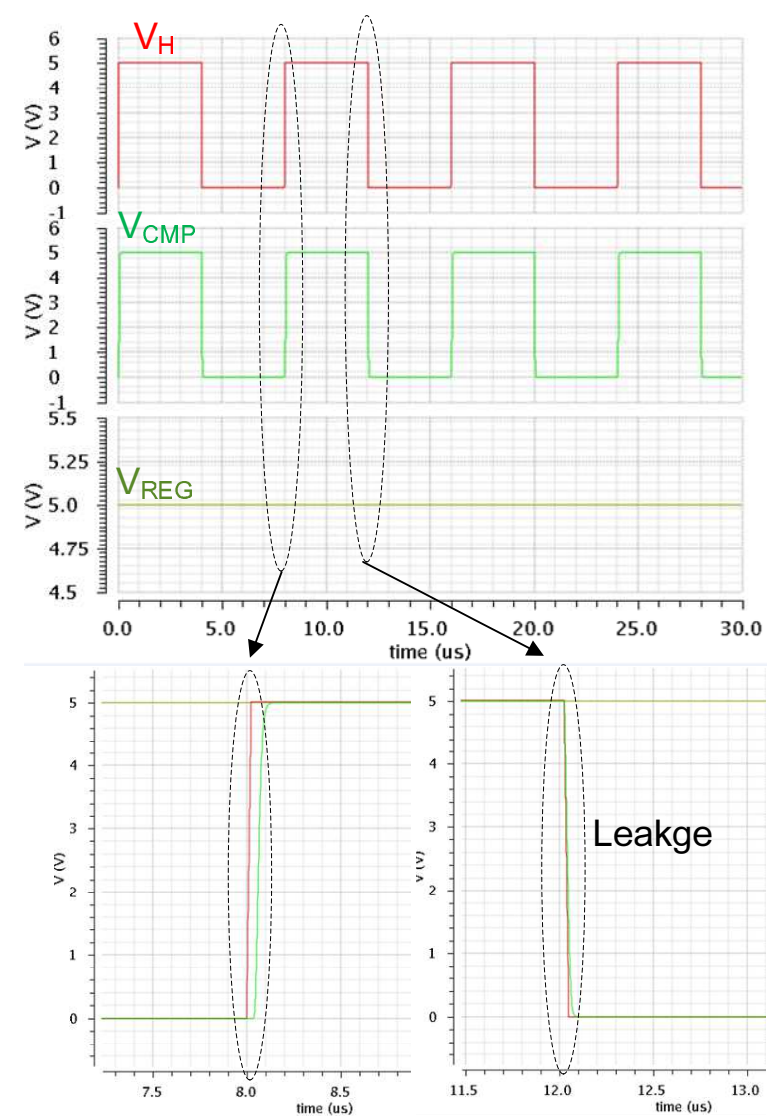

Figure 3.12: The leakage of the MOSFET.

\subsubsection{Ramp Generator}

The ramp generator of the PWM controller generates a sawtooth wave that determines the control frequency of the voltage regulator. In the proposed voltage regulator, the control frequency of $7.8 \mathrm{kHz}$ is not high, hence the feedback loop has high tolerance against the jitter performance of the control signal [86], [87], [88] and [89]. In that case, a phase-locked-loop (PLL) does not to be included.

The circuit of the ramp generator is shown in Figure 3.13. In this circuit, the sizes of $M P_{1}$ and $M P_{2}$ are the same while the size of $M N_{2}$ is nine times that of $M N_{1}$. So the rising slope of the Ramp is generated by charging a capacitor, $\mathrm{C}$, with a constant current, $I_{B I A S}$. Once the Ramp rises above $V_{+}$, the comparator, $C M_{1}$, triggers the R-S latch and sets the $S_{D c h g}$ to high voltage level. The $S_{D c h g}$ then turns off $M P_{3}$ and turn on $M N_{3}$, which discharges $\mathrm{C}$ with a constant current 


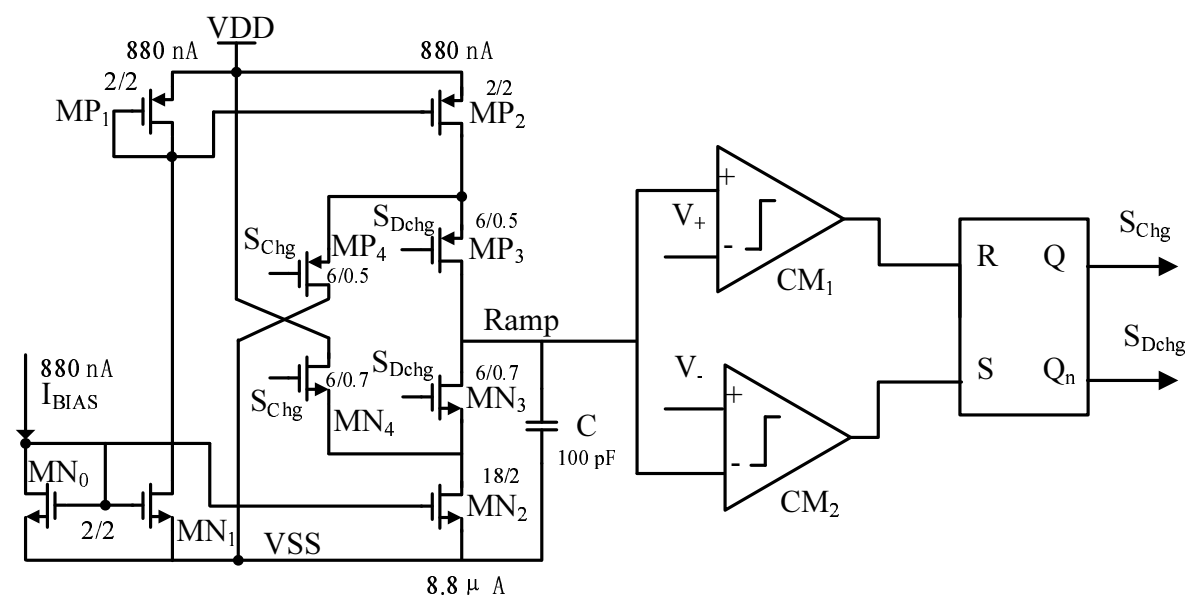

Figure 3.13: Schematic of Ramp generator.

which is equal to nine times that of $I_{B I A S}$. Once the Ramp falls below $V_{-}, C M_{2}$ triggers the R-S latch to turn off $M N_{3}$ and turn on $M P_{3}$. Switches $M N_{4}$ and $M P_{4}$ provide the current path when the current source transistors $M P_{2}$ and $M N_{2}$ are disconnected from $M P_{3}$ and $M N_{3}$ respectively so as to reduce the transient on-off glitches for the circuit [90].

The simulation results are shown in Figure 3.14. From this figure, the error voltage is very small, only $70 \mathrm{mV}$.

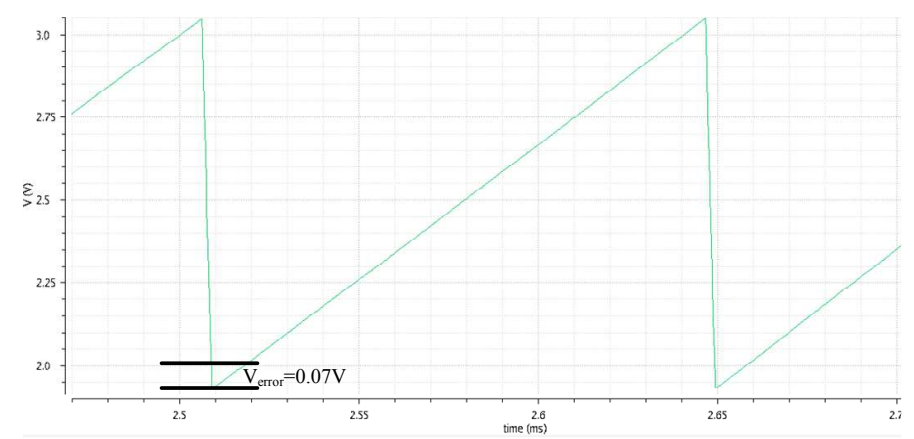

Figure 3.14: Simulation results of the ramp generator.

\subsubsection{Start-up Circuit}

The four internal diodes across the MOSFETs $N_{1}, N_{2}, P_{1}$ and $P_{2}$ work as a traditional passive full-bridge rectifier to regulate the $\mathrm{AC}$ input voltage to charge the $V_{R E G}$ up at the beginning. However, until the $V_{R E G}$ rises up to a certain value, the PWM control circuits cannot work accurately, which causes the failure of the 
regulator. A startup circuit is used to solve this problem. As shown in Figure 3.15, initially $V_{R E G}$ is small, hence $V_{f b 2}$ is lower than the Vth. So the start-up transistor is off and $V_{\text {start }}$ is clamped at $V_{R E G}$ by a pull-up resistor $R_{\text {pull }}$. $V_{\text {start }}$ forces the regulator to work in the charging configuration. When $V_{f b 2}$ is higher than $V_{t h}$, the NMOS is turned on to pull $V_{\text {start }}$ down to zero. After that, the regulator goes into steady state and the PWM controller takes charge to control the power stage. The power consumption of the start-up circuit is about $2 \mathrm{~mW}$.

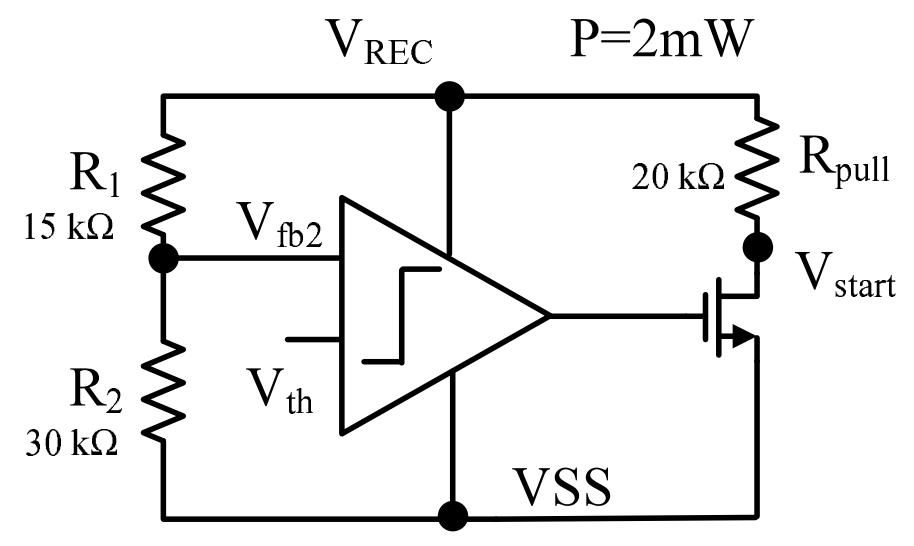

Figure 3.15: Schematic of Startup Circuit.

\subsubsection{Digital Controller}

The digital controller is shown in Figure 3.16. The first function of the digital controller is to determine the state of the regulator. If the " $V_{\text {start }}$ " is a highlevel voltage, the OR gate sets "Charging" to " 1 " and "Non-charging" to " 0 "; in this case, the digital controller forces the power stage to work in the charging configuration. If the " $V_{\text {start }}$ " is low, the digital controller let the PWM controller control the power stage.

The second function of the digital controller is to desynchronize the control signal and the driving signal of the main comparator $\left(C M P_{1}\right.$ and $C M P_{2}$ in Figure 3.10). As shown in Figure 3.10 (a), if the control switches $\left(S N_{11}, S N_{12}, S N_{21}\right.$ and $\left.S N_{11}\right)$ and power transistors $\left(N_{1}\right.$ and $\left.N_{2}\right)$ are turned on/off at the same time, there may be conflicting decisions. In this case, we do not want the control signals to switch at the same time as the main comparator, which turns on/off the power 


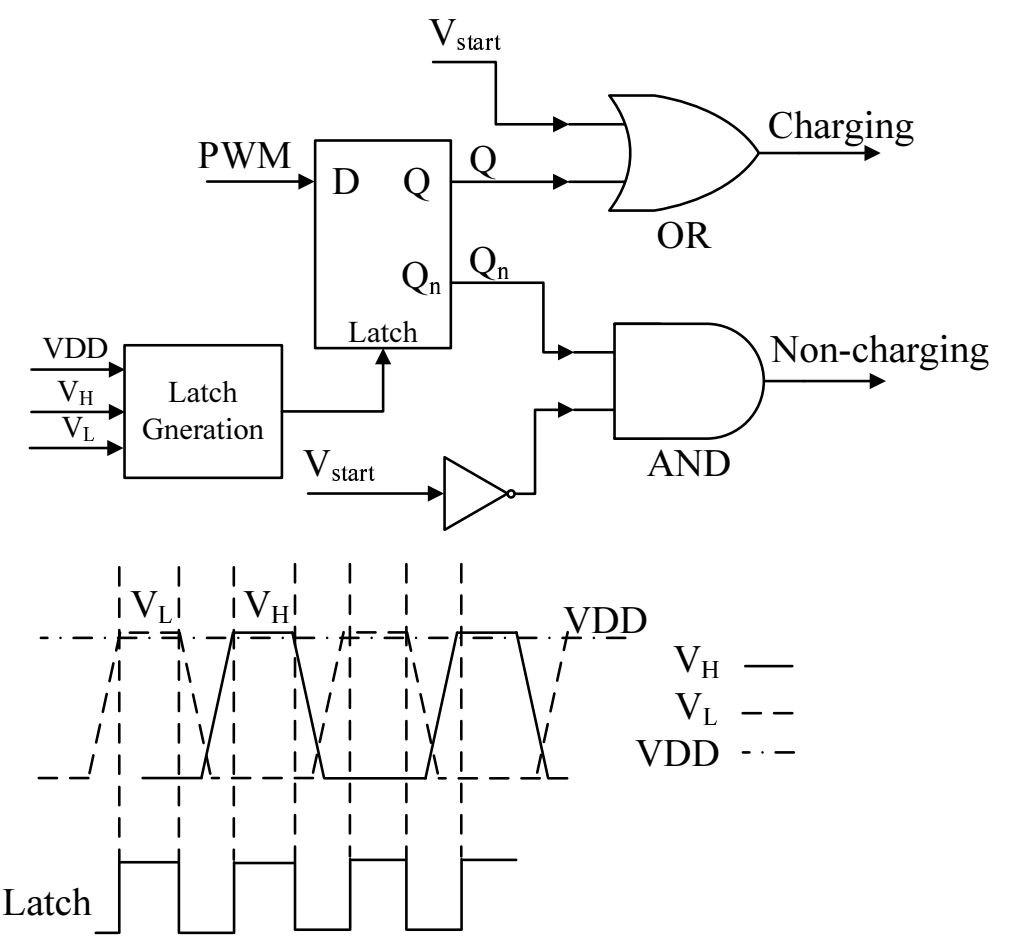

Figure 3.16: Schematic of Digital Controller.

MOSFETs at the rising/falling edge of the $V_{H}$ or $V_{L}$. In the proposed regulator, a latch generation is used to generate the control signal, which exclude the riding/falling edge of the $V_{H}$ and $V_{L}$. In that case, the conflicting actions would be avoided.

\subsection{Experimental Results}

The single-stage WPT voltage regulator (the power stage is not included) is fabricated in a $0.35 \mu \mathrm{m} \mathrm{CMOS} \mathrm{process.} \mathrm{The} \mathrm{die} \mathrm{photo} \mathrm{is} \mathrm{shown} \mathrm{at} \mathrm{the} \mathrm{top}$ of Figure 3.17 and the whole regulator that is fabricated on a PCB is shown at the bottom of Figure 3.17 (a). The power stage including four MOSFETs is highlighted. The measurement setup is shown in Figure 3.17 (b). With the proposed voltage regulator, a Class-D power amplifier operating at $125 \mathrm{kHz}$ with a constant 19 V DC input voltage and a WPT coil pair. The parameters of the charging coils are shown in Table 3.3. The WPT system works with the charging distance for the range between $4 \mathrm{~mm}$ and $8 \mathrm{~mm}$.

The charging frequency of the system is $125 \mathrm{kHz}$. The control switching 


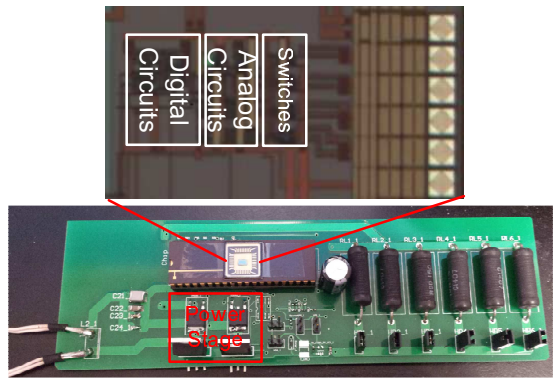

(a)

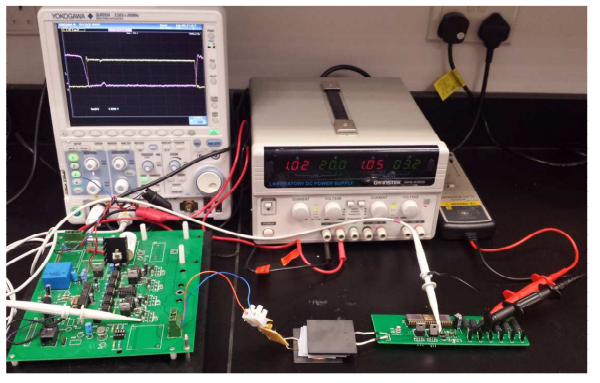

(b)

Figure 3.17: (a)Die photo and whole regulator on PCB.(b) Test bench

Table 3.3: Coil specifications

\begin{tabular}{|c|c|c|c|}
\hline & Diameter $(\mathrm{cm})$ & Inductance $(\mu \mathrm{H})$ & Q. at $125 \mathrm{kHz}$ \\
\hline Transmitting coil & 8.36 & 11 & 144 \\
\hline Receiving coil & 8.36 & 11 & 144 \\
\hline
\end{tabular}

frequency was designed to be $7.8 \mathrm{kHz}$. The $V_{R E G}$ is designed to $5 \mathrm{~V}$ to meet the requirement of most portable devices.

The load transition and the charging distance transition are shown in Figure 3.18. The waveforms for the load transition from $15 \mathrm{~W}$ to $2 \mathrm{~W}$ and vice versa at a charging distance of $4 \mathrm{~mm}$ are shown in Figure 3.18 (a) and (b) respectively. The waveform at a charging distance of $8 \mathrm{~mm}$ is shown in Figure 3.18 (c).

With regard to the regulator's performance, power conversion efficiency (PCE) is the most important index. It is defined as the ratio of $P_{O U T}$ to $P_{I N}$ of the regulator, where the former is the DC power consumed at $R_{L}$ while the latter is the $\mathrm{AC}$ power at the input to the regulator. The $\mathrm{AC}$ power is defined as the average value of product of the voltage $V_{H L}$ and $I_{a c}$ in Figure 3.10. The test is conducted with $R_{L}$ changing from $1.6 \mathrm{ohms}$ to $25 \mathrm{ohms}$ at a charging distance of $4 \mathrm{~mm}$. The result shown in Figure 3.19 indicates a $92 \%$ PCE at about 10 W. At light load, the efficiency drops below $70 \%$. A pie chart in Figure 3.19(b) and (c) shows the simulation result of power loss including non-charging configuration loss, conduction loss, switching loss and power consumption of the controller circuit. It can be seen that at the full load condition, the conduction loss due to the onresistance of the power device occupies most of the power loss while in the light 


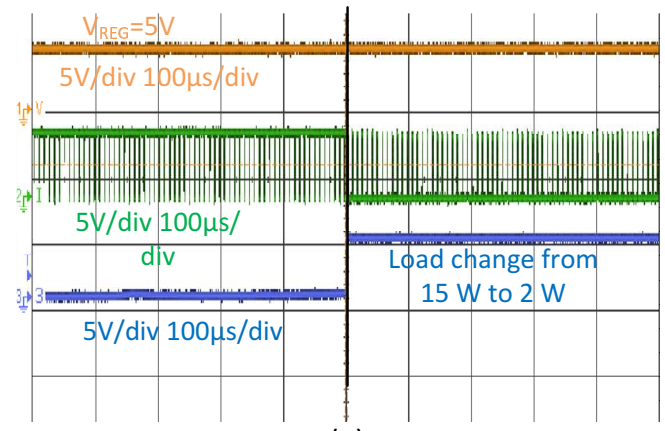

(a)

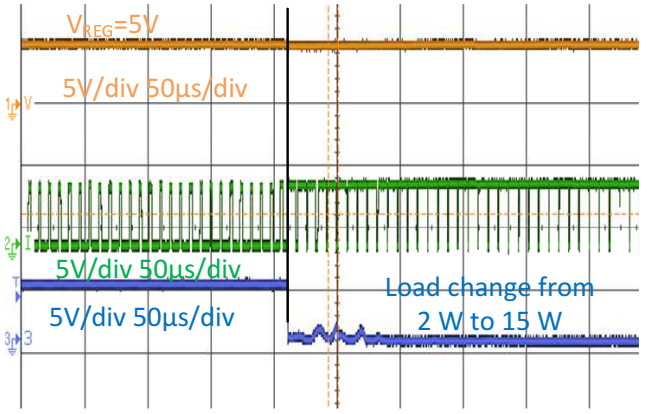

(b)

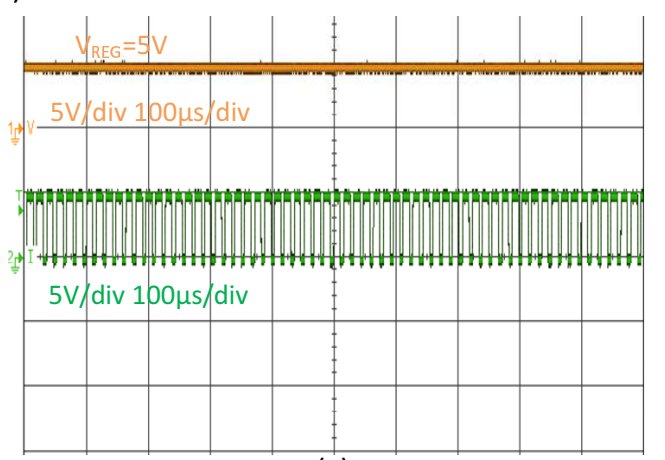

(c)

Figure 3.18: Oscilloscope waveform: (a) Measured waveform with a load transient of between $15 \mathrm{~W}$ and $2 \mathrm{~W}$ at $4 \mathrm{~mm}$; (b) Measured waveform with a load transient of between $2 \mathrm{~W}$ and $15 \mathrm{~W}$ at $4 \mathrm{~mm}$; (c) Measured waveform with a load of $15 \mathrm{~W}$ at $8 \mathrm{~mm}$.

load the non-configuration loss is the main power loss.

The load regulation, line regulation and the charging distance regulation are shown in Figure 3.20.

It can be seen from these figures that the voltage variation of the output voltage within reasonable range. For load regulation, from the figure we can see that
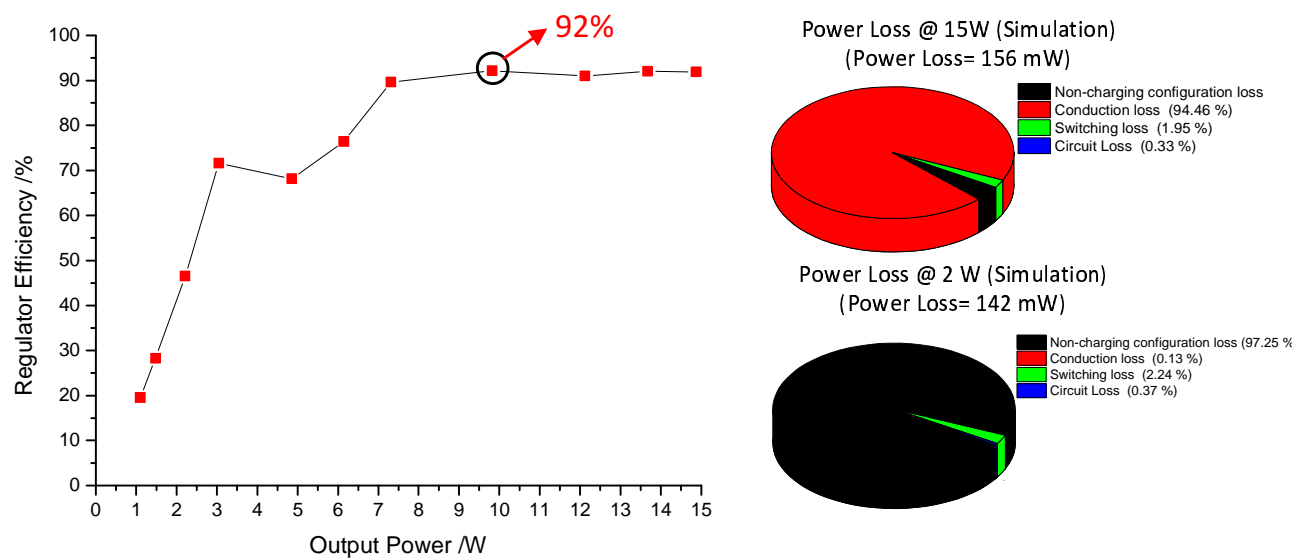

Figure 3.19: Measured efficiency of the regulator. 


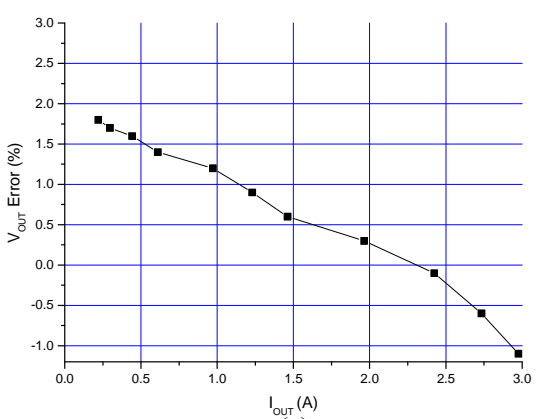

(a)

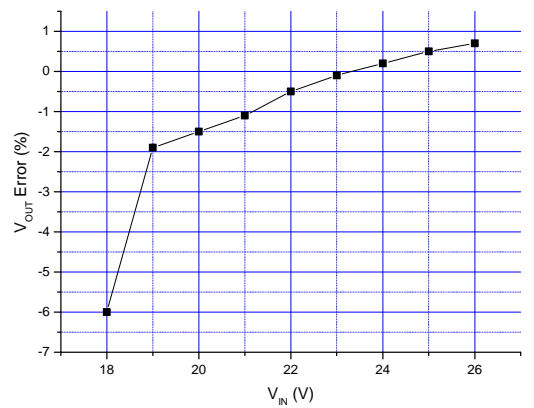

(b)

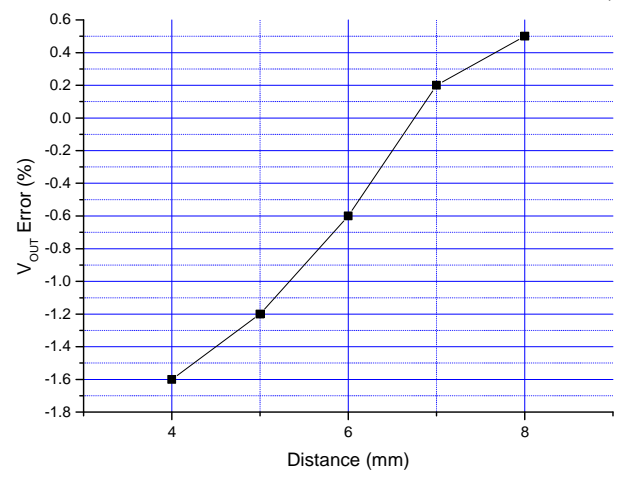

(c)

Figure 3.20: (a) Load regulation from $25(1 \mathrm{~W})$ to $1.6(15 \mathrm{~W})$ at $20 \mathrm{~V}$ input and $4 \mathrm{~mm}$ distance; (b) Line regulation from $18 \mathrm{~V}$ to $24 \mathrm{~V}$ at $15 \mathrm{~W}$ at $4 \mathrm{~mm}$; (c) Distance regulation from $4 \mathrm{~mm}$ to $8 \mathrm{~mm}$ at $20 \mathrm{~V}$ input and $15 \mathrm{~W}$.

for the entire range of the output current, the voltage variation is within $2 \%$; for line regulation, it can be seen from an input range of $19 \mathrm{~V}-26 \mathrm{~V}$, the voltage varies within $2 \%$, which means it can provide enough voltage however when the input voltage is only $18 \mathrm{~V}$, the regulator is not able to provide the desired voltage; for the transmission distance, if it is within $4 \mathrm{~mm}$ to $8 \mathrm{~mm}$, the voltage regulation is within $2 \%$. The voltage ripple is around $150 \mathrm{mV}$ to $200 \mathrm{mV}$.

Table3.4 summarizes the performance comparison with other WPT regulation system [10], [12] and [20]. This single-stage regulator has the largest $15 \mathrm{~W}$ output power for a wireless charger and it can regulate the output voltage without a communication circuit. Furthermore, the receiver efficiency is comparable with other works [34], [43] and [91].

The proposed architecture is verified in a $0.35 \mu \mathrm{m}$ standard CMOS process together with the power transistors on the PCB. External power transistors are used as BCD process is not available in author's research lab. This regulator is 
suitable for low cost portable applications requiring efficient robust WPT with a constant output voltage.

Table 3.4: Performance comparison with the proposed regulator

\begin{tabular}{|c|c|c|c|c|c|c|}
\hline & {$[34]$} & {$[43]$} & {$[91]$} & {$[92]$} & {$[93]$} & This Work \\
\hline Regulation & $\begin{array}{c}\text { Wire } \\
\text {-less } \\
\text { Comm. }\end{array}$ & $\begin{array}{c}3 \mathrm{R} \\
\text { circuit }\end{array}$ & $\begin{array}{c}\text { Wire } \\
\text {-less } \\
\text { Comm. }\end{array}$ & $\begin{array}{c}\text { Two } \\
\text { Stages }\end{array}$ & $\begin{array}{c}\text { Impedance } \\
\text { Matching }\end{array}$ & $\begin{array}{c}\text { Single } \\
\text { Stage } \\
\text { Regulator }\end{array}$ \\
\hline$V_{\text {in }}(\mathrm{V})$ & 19 & N.A. & N.A. & $6-12$ & $5-12$ & 19 \\
\hline$V_{\text {out }}(\mathrm{V})$ & 5 & 5 & 3.3 & 5 & 12 & 5 \\
\hline $\begin{array}{c}P_{\text {out }} \text { Max } \\
(\mathrm{W})\end{array}$ & 5 & 6 & 0.2 & 5 & 24 & 15 \\
\hline $\begin{array}{c}\text { Resonant } \\
\text { f. }(\mathrm{kHz})\end{array}$ & 125 & 6780 & $\begin{array}{c}100 \\
-150\end{array}$ & 144 & 200 & 125 \\
\hline $\begin{array}{c}\text { Receiver } \\
\text { Peak eff. } \\
(\%)\end{array}$ & 94 & 86 & $\begin{array}{c}\text { Around } \\
78\end{array}$ & 86 & 88 & 92 \\
\hline $\begin{array}{c}\text { Process } \\
\text { BCD }\end{array}$ & $\begin{array}{c}\text { BCD } \\
0.35 \mu \mathrm{m}\end{array}$ & PCB & $\begin{array}{c}\text { IC } \\
\text { N.A. }\end{array}$ & PCB & $\begin{array}{c}\text { CMOS } \\
0.35 \mu \mathrm{m} \\
\text { (off-chip } \\
\text { transistors })\end{array}$ \\
\hline
\end{tabular}

\subsection{Conclusions}

A novel AC-DC voltage regulator achieving low cost, high PCE has been presented. This WPT system has a power transfer capability of $15 \mathrm{~W}$ and an independently regulating ability without a step-down DC-DC converter and communication models. A cross-connected rectifier structure significantly increases the effic iency of the regulator. The maximum output power reaches $15 \mathrm{~W}$ and the peak efficiency of the receiver board is $92 \%$. The proposed architecture is verified in a $0.35 \mu \mathrm{m}$ standard CMOS process. This regulator is suitable for portable applications requiring efficient and intelligent WPT system. 



\section{Chapter 4}

\section{Design of A Full Active Rectifier}

\section{with Adaptive Time Delay Control \\ for 6.78 MHZ Wireless Power}

\section{Transmission}

\subsection{Introduction}

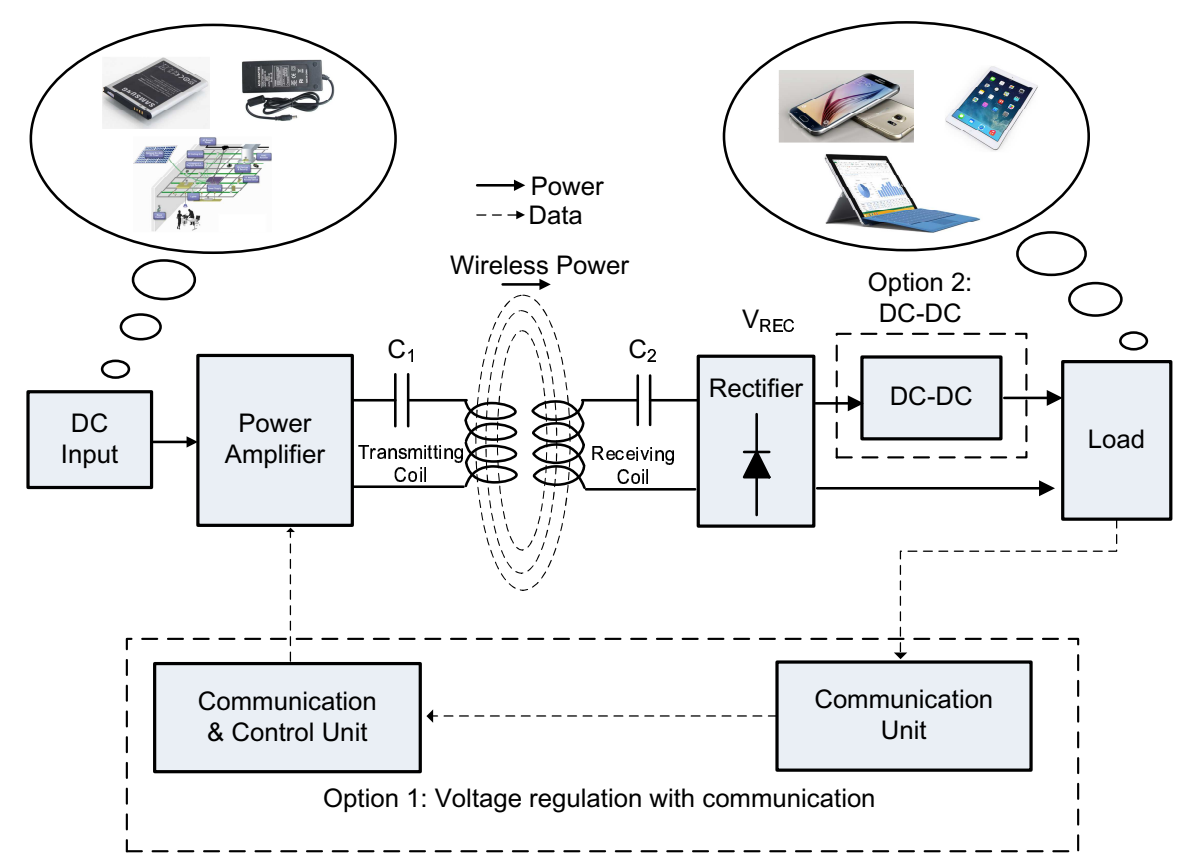

Figure 4.1: Wireless power transfer (WPT) system. 
Although the $\mathrm{kHz}$ wireless power transfer are popular. In order to extend its power transfer capability to higher power range (tens of Watts) and distance (e.g. several centimetres), the wireless power transmission system operating at the range of megahertz has recently been developed. It is particularly suitable to be used in tablets or video game consoles. The system topology of a WPT system is redrawn here, as shown in Figure 4.1. As can be inferred from this figure, the overall efficiency can be expressed as:

$$
\eta_{t o t}=\eta_{P A} \times \eta_{c o i l} \times \eta_{r e c} \times \eta_{D C}
$$

In line with Equation (4.5), it is discernible that rectifiers with a high efficiency can improve the overall performance. In other words, the device will then have a shorter charging time. Also note that the efficiency of a rectifier has a great impact on the thermal emission of a portable device. This means that a low power-loss rectifier has lower chances of becoming overheated by excessive resistive power loss. Nevertheless, while it is crucial to have a highly efficient rectifier, designing such rectifier with high output current for megahertz applications is a challenging task. This is because a rectifier with high output current requires its transistor sizes to be inevitably large in order to reduce the conduction power losses. This will in turn result in a longer turn on/off time, which is incompatible with high frequency applications [46], [47] and [48].

Full-wave rectifiers are widely used for wireless power transfer applications, because of their high voltage conversion ratio. In general, full-wave rectifiers can be divided into three categories: 1) passive rectifiers with four diodes, 2) half-active rectifiers [94], [95], and 3) full-active rectifiers [46], [47], [48] and [41]. For 1) and 2), the forward voltage of the diode limits the power efficiency and voltage conversion ratio of the rectifier. For 3), four power transistors are used as four respective switches with small on-state resistance, hence having a much lower power loss than that of the diodes. However, the techniques used for milliamps applications in [46]- [48] are not suitable for high power applications 
because of the large capacitances of the transistors. Delay locked loop (DLL) based on a voltage controlled delay circuit can be used to solve this problem [41]. To compensate for the delay of the gate driver, a replica delay unit has to be used in the DLL structure. Since the design is intended for high power application, the driver transistor itself can be very large; hence such replica unit would cause significant area overheads. Meanwhile, once there is a load change from high output current to low current, the active duration of the transistor would be shorter, which requires a fast response of the time delay response. However, it would take DLL tens of cycles to response, which would course the latent reverse current loss. In this chapter, we describe a ATD controller based on successive approximation register (SAR) algorithm to solve the above-mentioned problems. This solution is capable of extending the active duration of the rectifier while eliminating the reverse leakage.

The remaining of this chapter is organized as follows: 4.2 describes the architecture and operating principles of the proposed full-active rectifier; detailed circuit blocks such as the rising/falling edge detector, proximity detector, and the time-to-digital conversion circuit are described in 4.3; experimental results are shown in 4.4; whereas 4.5 draws the conclusions.

\subsection{Proposed Rectifier with Adaptive Time Delay (ATD) Con- trol}

\subsubsection{Structure and Operating principles of the Proposed Rectifier}

The topology and operating principle of a basic cross-connected full active rectifier with the ATD controller is shown in Figure 4.2. The magnetic flux arising from the PA produces an $\mathrm{AC}$ voltage, $V_{A C}$ (refer to Figure 4.1). $C_{D C}$ denotes the DC smoothing capacitor and $R_{L}$ is the load resistor. Two GaN transistors, $N_{3}$ and $N_{4}$, with low voltage drop, connected in a cross-coupled configuration act as power switches turned on/off by $V_{A C}$. In addition to the switch pair, in order to 


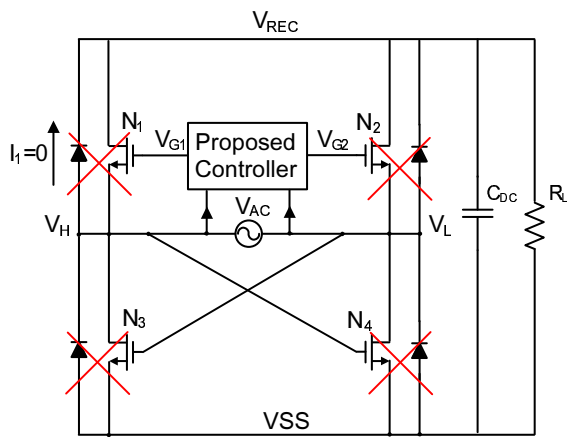

(a) $\mathrm{V}_{\mathrm{AC}}<\mathrm{V}_{\text {th }}$

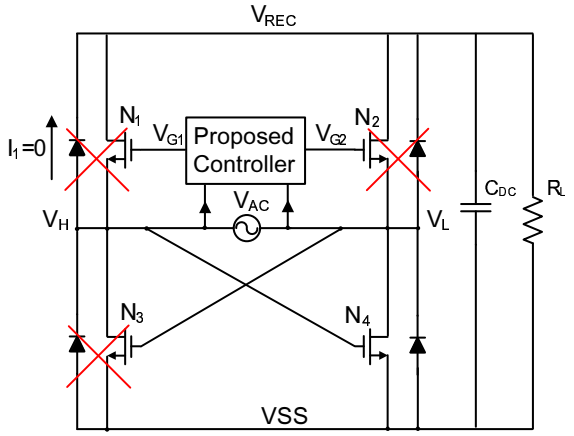

(b) $\mathrm{V}_{\mathrm{th}}<\mathrm{V}_{\mathrm{AC}}<\mathrm{V}_{\mathrm{REC}}$

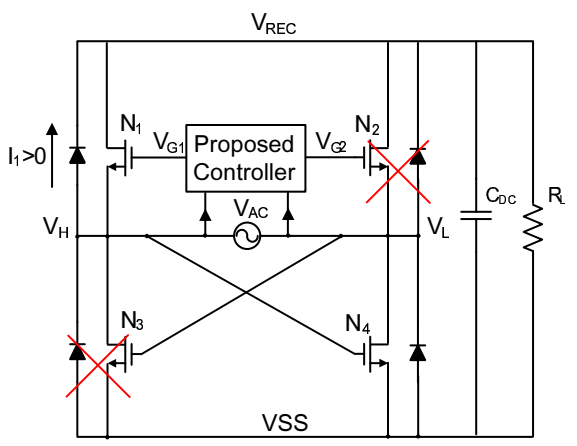

(c) $\mathrm{V}_{\mathrm{REC}}<\mathrm{V}_{\mathrm{AC}}$

Figure 4.2: Topology and operation of the proposed active rectifier.

avoid the reverse leakage current from the load to the source, another two GaN transistors, $N_{1}$ and $N_{2}$, are controlled by an ATD controller, which turns on $N_{1}$ and $N_{2}$ only when $V_{A C}$ is larger than $V_{R E C}$. The ATD controller monitors and controls the time difference between the gate drive signals $\left(V_{G 1}\right.$ and $\left.V_{G 2}\right)$ and the input voltages $\left(V_{H}\right.$ and $\left.V_{L}\right)$. A SAR algorithm is employed to maximize the conduction time of $N_{1}$ and $N_{2}$ to enhance the efficiency.

The operating principle of the rectifier during steady-state for the positive half cycle is illustrated in Figure 4.2. At the beginning of the positive half cycle (Figure 4.2 (a)), the input $\mathrm{AC}$ voltage, $V_{A C}$, has not yet reached the threshold voltage level of the $\mathrm{GaN}$ transistor, $V_{t h}$. In other words, when $0<V_{A C}<\left|V_{t h}\right|$, 
$N_{4}$ is "off" and $N_{1}$ is also turned off by the controller, there is no current going through $N_{1}$ and $N_{4}$. When $V_{A C}$ exceeds $V_{t h}$, i.e. $V_{A C}>\left|V_{t h}\right|, N_{4}$ will be turned on, causing $V_{L}$ to be equal to VSS, whereas $N_{1}$ is still turned off, meaning that the current flows through $N_{4}$ and diode of $N_{1}$. If $V_{A C}$ continues to increase, since $V_{L}$ is now connected to VSS by $N_{4}$, the rising $V_{A C}$ will be further pulled up to $V_{H}$. Once $V_{A C}$ exceeds $V_{R E C}$, the controller will begin to turn on $N_{1}$. Consequently, due to the conduction of $N_{1}$ and $N_{4}$, current begins to flow from $V_{A C}$ to the load the smoothing capacitor $C_{D C}$. Since $N_{1}$ and $N_{4}$ are two GaN devices working in the deep triode region, their on-state resistance is small, which can help to minimize the conduction power loss, thus increasing the efficiency of the rectifier. $V_{A C}$ begins to drop when it reaches its peak value. The controller sets a time delay to turn off $N_{2}$ exactly when $V_{A C}<V_{R E C}$ as well as then there is no current going through $N_{1}$. After once $V_{A C}$ decreases to $V_{t h}$, both $N_{1}$ and $N_{4}$ are turned off and the positive half cycle will not be terminated until $V_{A C}$ drops to zero.

The conduction time of $N_{1}$ is controlled by the ATD controller, as shown in the Figure 4.2. If the conduction time is insufficient, $N_{1}$ is turned on at first such that the parallel diode can then conduct the current before $V_{A C}$ drops to $V_{R E F}$. The power losses in the diode deteriorate the performance of the rectifier. On the other hand, if the conduction time is too long, $N_{1}$ may not be fully turned off when $V_{A C}<V_{R E C}$. As a result, a reverse leakage current will flow from $V_{R E C}$ to $V_{A C}$, thus causing a high power loss.

The operating principle of the negative half cycle is similar to that of the positive half-cycle except that in the negative half cycle the current flows through $N_{2}$ and $N_{3}$.

\subsubsection{Adaptive Time Delay Control Algorithm}

Rectifiers reported in [46] and [47] use active switches controlled by highspeed comparators to eliminate the reverse leakage current. The basic idea is that the high-speed comparator can shut off the transistor once $V_{A C}$ decreases to 
$V_{R E C}$. Since the propagation time of the comparator is very short, it can prevent the leakage current from flowing through the transistor completely. However, this method is suitable only for those applications with small output current, e.g. in the range tens to hundreds of milliamperes. For wireless power transmission in cell phones or tablets, the large output current needs be handled by large-size power transistors, the input capacitance of which is expected to be several times of those of low power application. As a result, when $V_{A C}$ decreases to just below $V_{R E C}$, the comparator cannot turn off the upper side transistors $\left(N_{1}\right.$ and $\left.N_{2}\right)$ in a short given time. Taking the positive half cycle as an example, both $N_{1}$ and $N_{4}$ are still turned on when $V_{A C}$ drops just below $V_{R E C}$, in this case, a reverse current hence flows from $C_{D C}$ back to the rectifier. Consequently, the power loss due to such a leakage current may be detrimental. In [48], an unbalanced-biased comparator design is utilized to compensate the turn off time of the transistor. However, the input $\mathrm{AC}$ voltage $V_{A C}$ in a high power wireless power transmission application is more of a square wave than a sinusoidal wave. This implies that the unbalanced-biased comparators may cause instability issues.

Figure 4.3 shows the topology of the proposed rectifier with the ATD controller, and Figure 4.4 shows the timing diagram of the controller. In the proposed rectifier, the duty cycle of the gate drive signal $t_{d(n)}$ of $N_{1}$ and $N_{2}$ is adjusted to compensate the propagation time of the gate driver such that the transistor will be turned off exactly when $V_{A C}$ decreases to $V_{R E C}$, so that to remove the reverse current. A controller is used to detect the time difference between the transistor shut off time and the moment when $V_{A C}$ decreases to $V_{R E C}$, which is represented by $\Delta \mathrm{t}$, as shown in Figure 4.4. A successive approximation feedback algorithm is utilized to control the duty cycle of the transistor. Now consider the positive half cycle $\left(V_{A C}=V_{H}-V_{L}>0\right)$ of the rectifier: As $V_{H}$ increases above $V_{R E C}$, the ATD controller commands $S_{1}$ to generate a setting signal to trigger the SR latch to turn on $N_{1}$. In the n-th cycle, an adjustable time delay is set to generate a resetting signal, $R_{1}$, to trigger the SR latch to turn off $N_{1}$ after an elapse of $t_{d(n)}$, which 


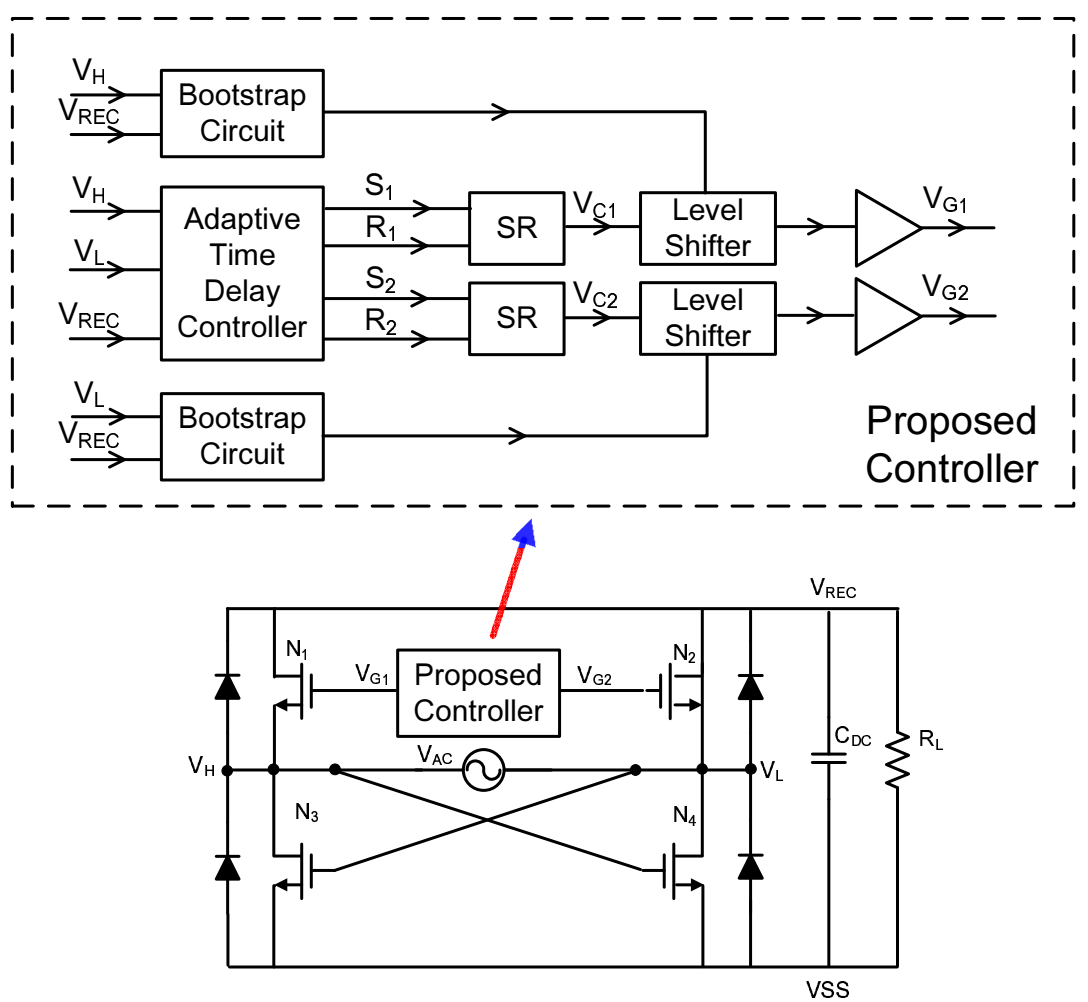

Figure 4.3: Details of the adaptive time delay control.

equals $t_{f i x}$, if $\mathrm{n}=0$. After gate propagation, $t_{p}, N_{1}$ is then fully turned off. The ATD control module records the $t_{d(n)}$. In the next cycle, the control module sets the duty cycle of $N_{1}$ as

$$
t_{d(n-1)}=t_{d(n)}+\frac{1}{2} \times \Delta t_{(n)}
$$

This iteration will continue until $\Delta \mathrm{t}$ closely approaches zero after several cycles. When the iteration completes, the controller locks the time delay, and the rectifier then works in a steady state. It is worth noting that the additional propagation delay for the turn-on of $N_{1}$ is acceptable, because it does not generate any reverse leakage current.

The full active rectifier with ATD control has the following advantages. First, the SAR algorithm can gradually align the edge of the gate drive signal to the edge of the AC voltage, which maximizes the conduction time of the GaN transistors and in turn increases the efficiency. Second, the time delay lock function circuits guarantee that the total delay of the circuit does not push the edge of the gate 


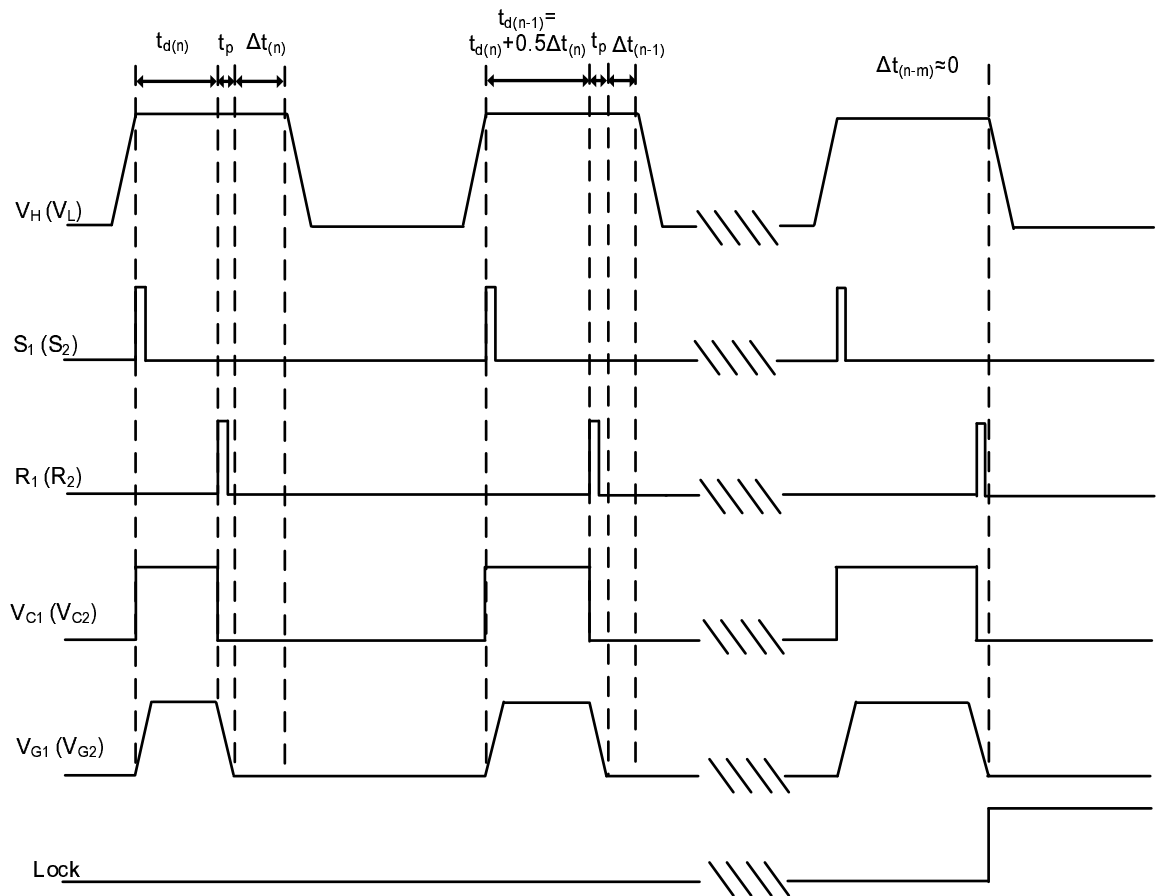

Figure 4.4: Timing of the adaptive time delay control.

drive signals after the $\mathrm{AC}$ voltage, which eliminates the leakage current of the rectifier.In addition, in this design, the controller automatically detects both the $\Delta \mathrm{t}$ and the total time delay thus further protecting the circuit from the reverse leakage.

\subsubsection{Start-up Mechanism}

At the very beginning, $V_{R E C}$ has not been powered up by wireless power from the transmitter; both the input AC voltage and the output DC voltage are close to $0 \mathrm{~V}$, hence causing the controller to work in a unstable state, which is undesirable. It is therefore important to ensure that the proposed rectifier is able to startup in such a low voltage condition [96], [97], [98] and [99].

The timing diagram during start-up of the proposed active rectifier is shown in Figure 4.5. When the transmitter begins to transfer wireless power, the receiving coil generates an input AC voltage for the rectifier. However, the initial operating points of the $V_{R E C}$ are too low to activate both the control circuit and the GaN transistor. At the same time, the diodes form a passive full bridge diode rectifier 


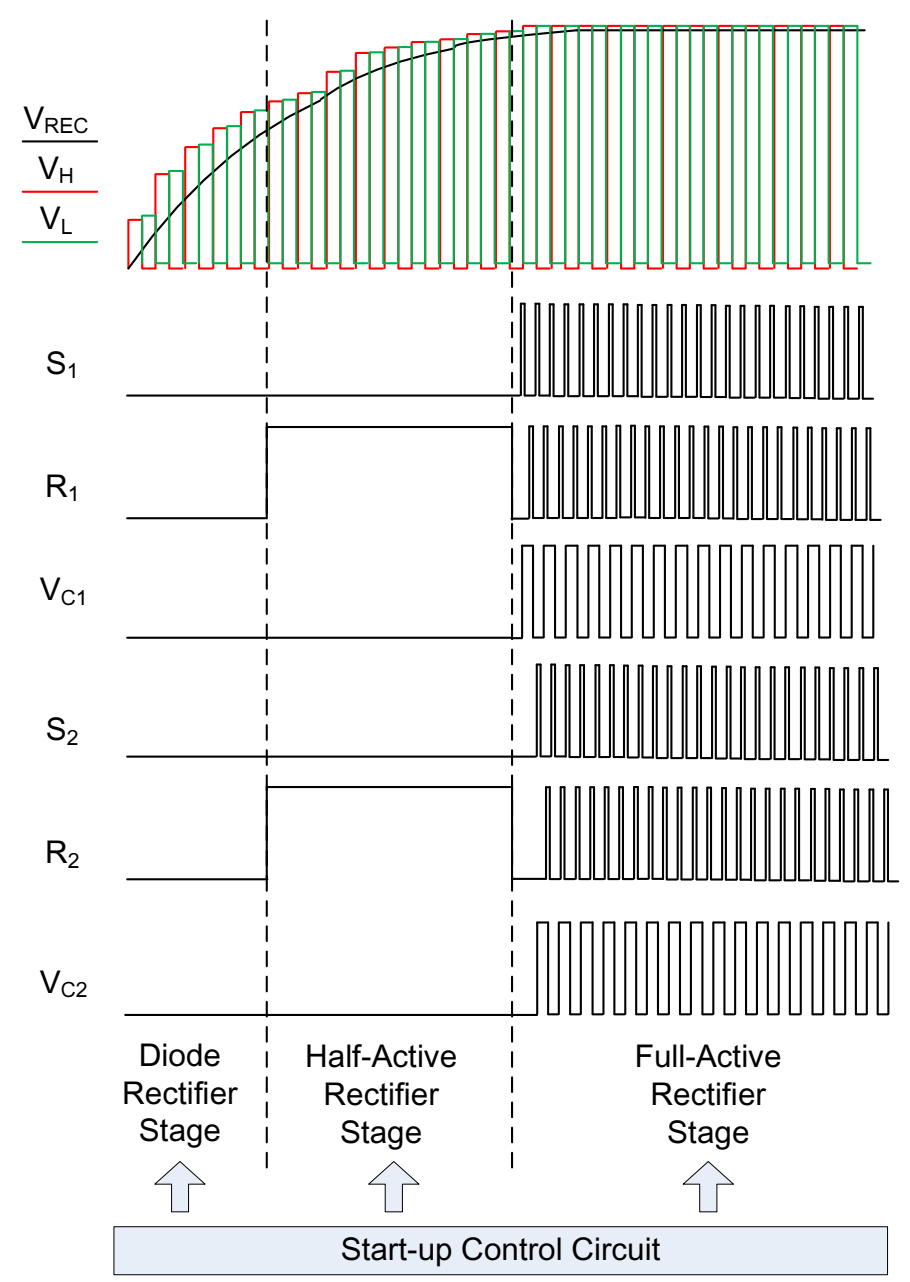

Figure 4.5: Timing diagram of the rectifier at start-up.

and start to charge up the load.

After the input voltage reaches the threshold voltage of the GaN devices, transistors $N_{3}$ and $N_{4}$ can then be turned on by $V_{A C}$. However, the $V_{R E C}$ at this stage is still too low to activate the controller. As a result, the rectifier can only work in the half-active mode. In such a mode, it is worth noting that the gate voltages of $N_{1}$ and $N_{2}$ should be set to " 0 ", since the controller would give out unpredictable output with a low power supply. In view of this, the start-up control circuit sets $S_{1}, S_{2}$, to " 0 " and $R_{1}, R_{2}$ to " 1 ", making sure that the rectifier to work in the half-active mode, as shown in Figure4.5. The rectifier will continue to operate in the half-active mode until $V_{R E C}$ is charged up to a level, at which all remaining control circuits can work predictably.

As long as $V_{R E C}$ is at a proper level as discussed above, the rectifier will work 
well in the full-active mode. In this mode, the adaptive controller starts to control the duty cycle of $N_{1}$ and $N_{2}$ as an active switch.

\subsection{Circuit Structure and Key Blocks}

\subsubsection{Topology of the Proposed Rectifier}
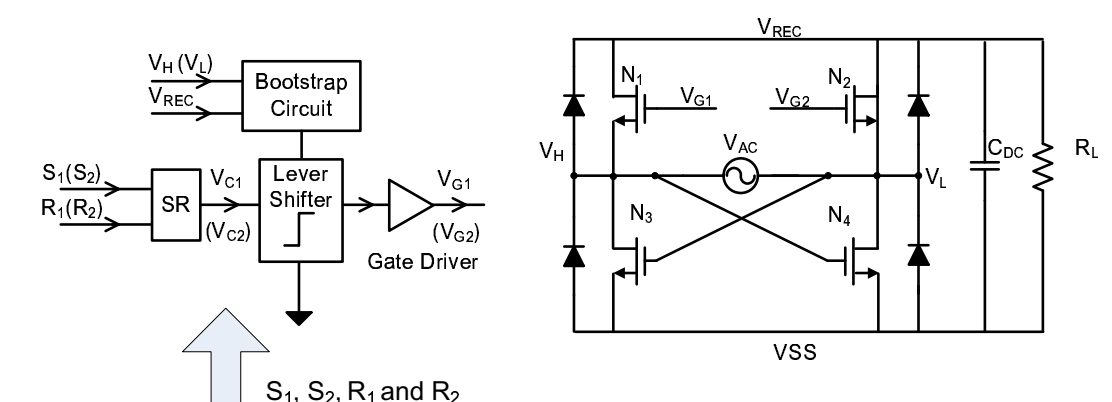

$S_{1}, S_{2}, R_{1}$ and $R_{2}$

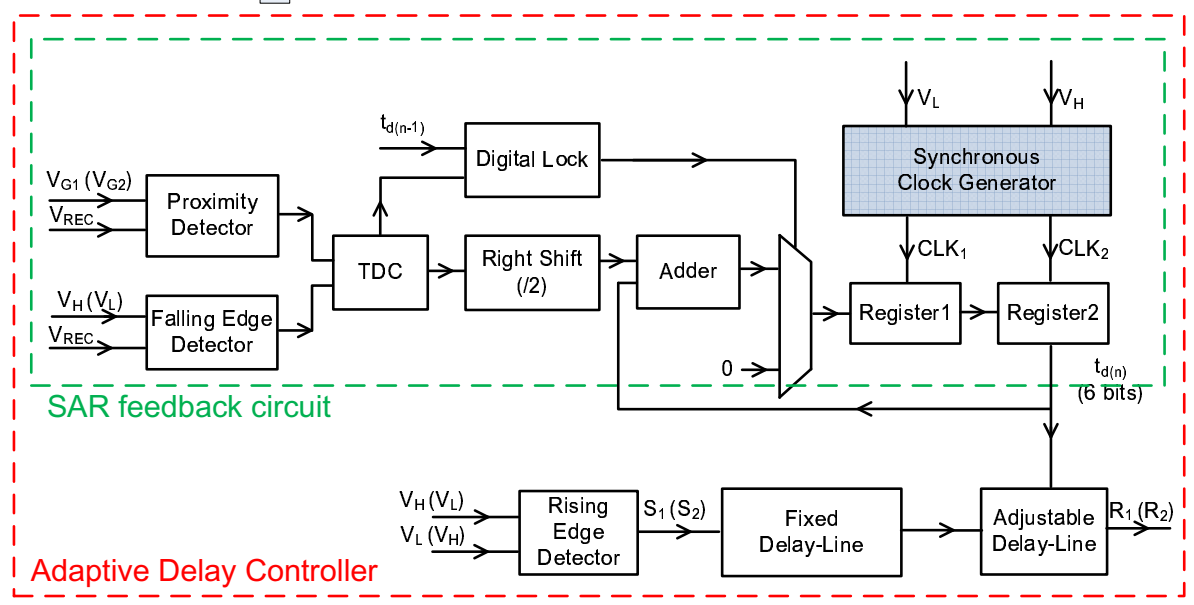

Figure 4.6: Topology of the proposed rectifier with detailed blocks.

The proposed active rectifiers along with all detailed blocks are illustrated in Figure 4.6. To meet the requirements of the high power devices, the rectifier is designed to support a $15 \mathrm{~W}$ output at $5 \mathrm{~V}$ so as to provide rapid charging for devices such as cell phones and tablets. The resonance frequency of the wireless power transmission system is targeted at $6.78 \mathrm{MHz}$ for mid-distance applications.

The ATD controller marked in red dashed lines as shown in Figure 4.6 were fabricated based on a $0.18 \mu \mathrm{m}$ high-voltage AMS process. The GaN power transistors $\left(N_{1}-N_{4}\right)$ are not incorporated in this chip design due to the integrability issues. As described earlier, the lower side GaN FETs $\left(N_{3}\right.$ and $\left.N_{4}\right)$ are self-turned 
on/off by the AC input voltage, whereas the upper side GaN FETs $\left(N_{1}\right.$ and $\left.N_{2}\right)$ are controlled by the ATD controller. The ATD controller consists of an SAR feedback circuit, a fixed time delay unit and an adjustable time delay unit. For $N_{1}$ and $N_{2}$, level shifters are used to boost the voltage level of the control signal. The gate drive circuit is used to provide a large gate charging/discharging current for $\mathrm{GaN}$ devices with associated large parasitic capacitance.

The SAR feedback circuit is composed of a rising edge detector, gate proximity detector, falling edge detector, time-to-digital converter (TDC), right shift, adder, registers, and digital lock circuit.

Since $N_{1}$ and $N_{2}$ should be controlled separately, typically two sets of controller and delay units need to be used. However, since only one upper side transistor (either $N_{1}$ or $N_{2}$ ) conducts current in one single half period, the power consumption of the controller are therefore limited.

As shown in Figure 4.6. The proximity detector generates a step signal once the gate voltage falls to the AC voltage (either $V_{H}$ or $V_{L}$ ), while the falling edge detector generates a step signal once $V_{A C}<V_{R E C}$. The TDC measures the time difference between these two step signals. The digital circuits including a right shift, an adder, and two registers realize the function described in Equation (4.2). The synchronous clock generator unit is used to switch the TDC and other digital circuits in different cycles. The digital result of delay time is stored in Register2, as shown in Figure 4.6. Once the $\Delta \mathrm{t}$ reach the set threshold value, the input of time delay line will be locked and the TDC keep monitoring the $\Delta \mathrm{t}$ in order to protect the circuit from potential reverse leakage current.

\subsubsection{Rising Edge Detector and Falling Edge Detector}

The rising and falling edge detector [100], [101], and [102] are used to detect the moment when $V_{A C}$ crosses $V_{R E C}$. For the rising edge detector, it is desired to have a fast response when $V_{A C}$ surpasses $V_{R E C}$ to maximize the conduction time of $N_{1}$ or $N_{2}$. For the falling edge detector, the response should be fast as well, to 
ensure more accurate $\Delta$ t detection.

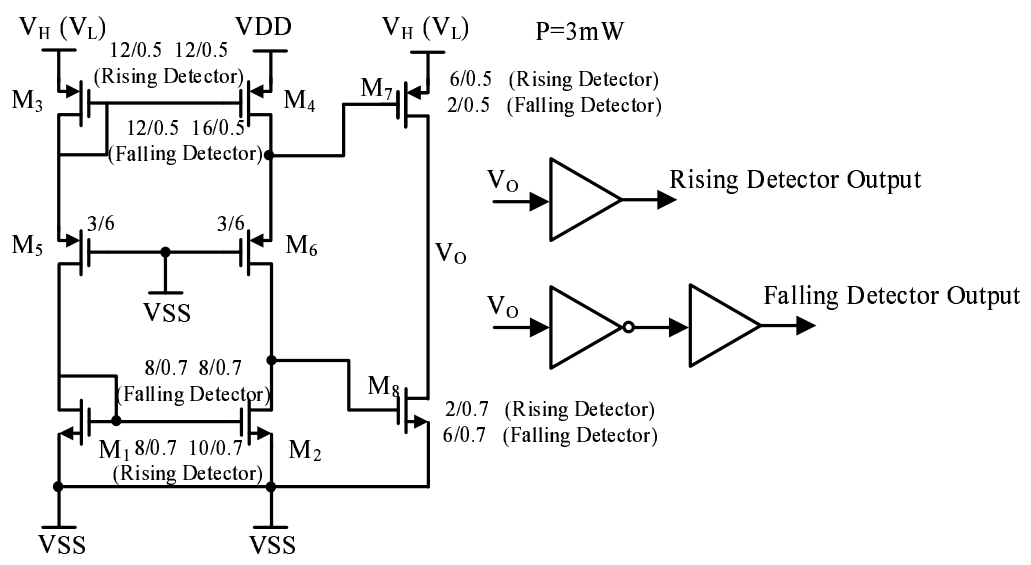

Figure 4.7: Topology of the rising and falling edge detector.

The widely used common-gate comparator is chosen to construct the rising and falling edge detector. The schematic of the detector is shown in Figure 4.7. Compared to the common-source comparator, the common-gate structure will suffer less from the input offset voltage. The detector has three inputs: $V_{H}$ or $V_{L}$, $V_{R E C}$ and VSS. For the rising edge detector, the output is set to 1 if the input AC voltage $\left(V_{H}\right.$ or $\left.V_{L}\right)$ signal is larger than $V_{R E C}$-VSS. As for the falling edge detector, it provides the output signal only when the input $\mathrm{AC}$ voltage is smaller than $V_{R E C}$. Consider now the positive half cycle: When the AC voltage $\left(V_{H}\right.$ or $\left.V_{L}\right)$ increases above the level of $V_{R E C}$, the gate voltages of $M_{3}$ and $M_{1}$ will correspondingly increase to a certain level, at which $M_{4}$ is turned off and $M_{2}$ is turned on. As a result, $M_{7}$ is turned on and $M_{8}$ is turned off. This generates a high $V_{O}$.

When $V_{H}$ or $V_{L}$ decreases below $V_{R E C}, M_{4}$ is turned on and $M_{2}$ is turned off. This generates a low $V_{O}$. An inverter is thereby added for the falling edge detector. Additional drivers are added to drive the input capacitance associated with the post-processing circuits. Transistors $M_{5}$ and $M_{6}$ specifically serve as bias-limiting transistors for this high voltage application. They guarantee a small dead-time between the states of on and off so as to eliminate the shoot-through current during the transient period.

In order to speed up the transient response of the detector, the size of $M_{2}$ is designed to be larger when compared to $M_{1}$ to improve the pull-down response 
for the rising edge detector, and the same goes for transistor $M_{7}$; For the falling edge detector, on the contrary, larger sized transistor $M_{4}$ and $M_{8}$ are used to improve the response when $V_{A C}$ decreases below $V_{R E C}$. The power consumption of the rising/falling detector is about $3 \mathrm{~mW}$.

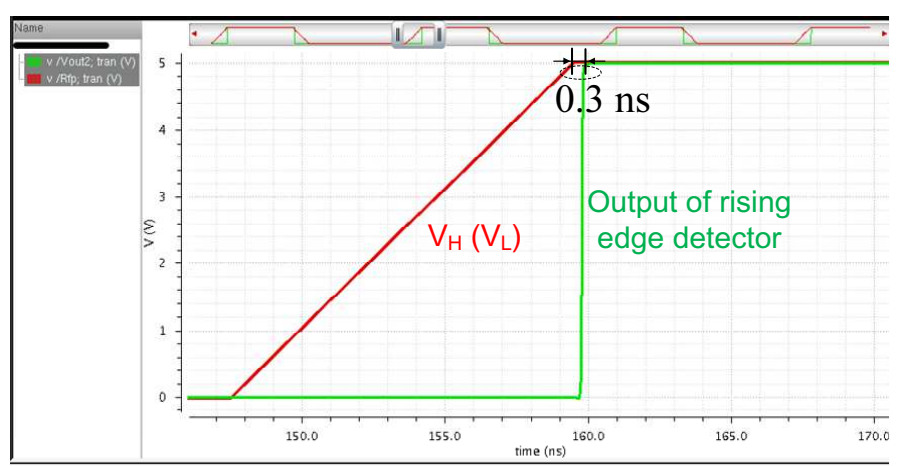

Figure 4.8: Simulation results of the rising edge detector.

The simulation results are shown in Figure 4.8 and Figure 4.9. It can be seen both detectors can have a fast response when the rising or falling edge come. In the simulation, the amplitude of both the input is determined by the consideration of the current and the on-resistance of the GaN devices. The frequency of the input is $6.78 \mathrm{MHz}$ and the rising and falling time for the input pulse is around 12 ns due to the simulation results of the rectifier. The response time of the rising edge detector is around $0.3 \mathrm{~ns}$, while the response time of the falling edge detector is $0.8 \mathrm{~ns}$.

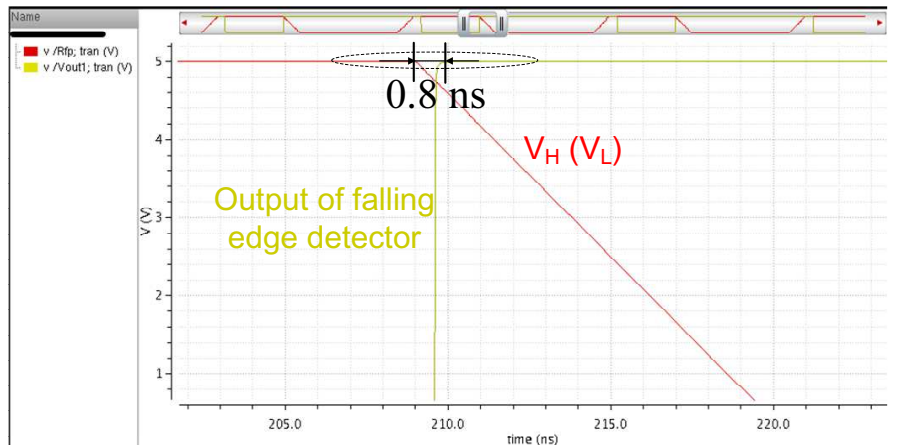

Figure 4.9: Simulation results of the falling edge detector. 


\subsubsection{Proximity Detector}

The proximity detector is used to detect the very moment the gate driving signal falls to $V_{R E C}$. At this time instance, the transistor is fully shut off. The proximity detector also provides the input signal for TDC. Figure 4.10 depicts the structure of the proposed detector, which consists of two unbalanced bias current $\left(I_{S 1}\right.$ and $\left.I_{S 2}\right)$, a pair of current amplifier with two current mirrors $\left(M_{9}\right.$, $M_{10}$ and $\left.M_{13}, M_{14}\right)$, and a buffer used to drive the post-processing circuit. In the proposed detector, the branch consisting of $M_{13}$ and $M_{15}$ acts as a push-pull stage to accelerate the charging and discharging of the node capacitance.

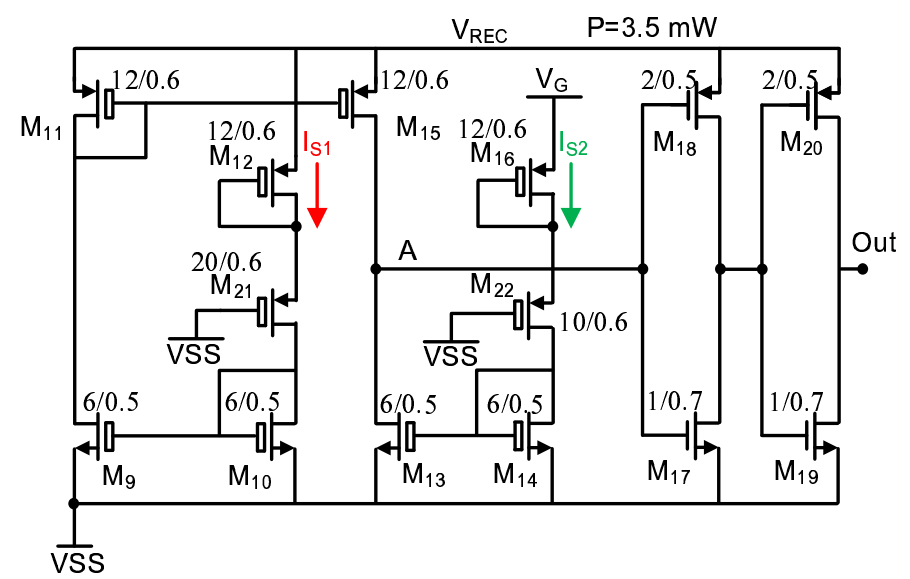

Figure 4.10: Scheme of the gate proximity detector.

The proposed unbalanced-biased current generator is used to create a voltage bias. If the sizes of transistors $M_{14}$ and $M_{16}$ are the same as those of $M_{10}$ and $M_{12}$, the currents in $M_{15}$ and $M_{13}$ will be balanced. In that case, when $V_{G}$ drops to $V_{R E C}$, node A will not be pulled up to $V_{R E C}$. The actual circuit function is realized by setting $I_{S 1}>I_{S 2}$ so that the detector is able to pull the node A high as $V_{G}$ approaches $V_{R E C}$. The difference between $I_{S 1}$ and $I_{S 2}$ is controlled by setting the size of $M_{21}$ to be larger than that of $M_{22}$. As a result, the pull-up capability of $M_{21}$ is stronger than that of $M_{22}$, thus causing the pulling up current of the $M_{15}$ larger the pulling down current of $M_{13}$ when the $V_{G}$ comes down below $V_{R E C}$.

If $V_{G}$ is much larger than $V_{R E C}$, it is apparent that $I_{S 1}$ is then much smaller than $I_{S 2}$. $I_{S 2}$ is mirrored by the current mirror which consists of $M_{13}$ and $M_{14}$. 
The difference between the two currents has a push-pull effect, which can pull node A down to ground. As $V_{G}$ decreases to the vicinity of $V_{R E C}$, due to the size difference of $M_{12}$ and $M_{16}, I_{S 1}$ will be larger than $I_{S 2}$. The current of $I_{S 1}-I_{S 2}$ thereby begins to charge the capacitance at node A, which pulls $\mathrm{A}$ to a higher voltage level to turn on the buffer and set the voltage of the output of the detector to be high. It should be pointed out that like the circuit in Figure 4.7, the limiting transistors are used in this circuit as well. However, since signals for $\mathrm{GaN}$ devices are over $5 \mathrm{~V}$, so high voltage transistors $\left(M_{9}-M_{16}\right)$ have to be used in this design. Usually, a high voltage transistor has a larger threshold voltage compared to normal transistors. As a result, the resistance of the limiting transistors, $M_{21}$ and $M_{22}$, should be designed smaller so that they will not slow down the response of the detector. The power consumption of the rising/falling detector is about 3.5 $\mathrm{mW}$.

The simulation results are shown in Figure 4.11. It can be seen that the proximity detector can have a fast response when the falling edge of the gate driving signal came. In the simulation, the amplitude of the gate drive signal was $10 \mathrm{~V}$ - $0 \mathrm{~V}$ at a frequency of $6.78 \mathrm{MHz}$. The response time of the rising edge detector is around $5 \mathrm{~ns}$, while the response time of the proximity detector is $0.6 \mathrm{~ns}$. This time delay is the reason that why the cross-connected rectifier can not be used in this design.

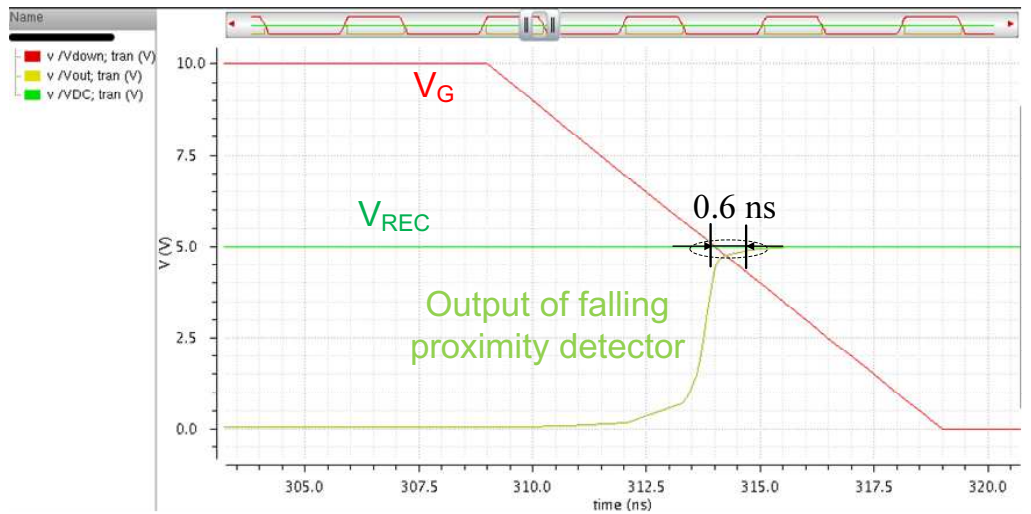

Figure 4.11: Simulation results of the proximity detector. 


\subsubsection{Digital Blocks}

The digital blocks serve three main purposes: data conversion, calculation and recording. The data conversion circuit is carried out by TDC, whose structure is shown in Figure 4.12. In this design, a basic digital delay-line based TDC is constructed( [103], [104], [105] and [106]). The "start" signal is delayed by a delay-line consisting of 64 delay elements. Those delayed signals are sampled in parallel, once the"stop" signal arrives. The output of this TDC is a sequence of thermometer codes. To help the post-processing circuit to deal with such data, a thermometer to binary decoder is designed to transfer the output to a 6 bit binary code.
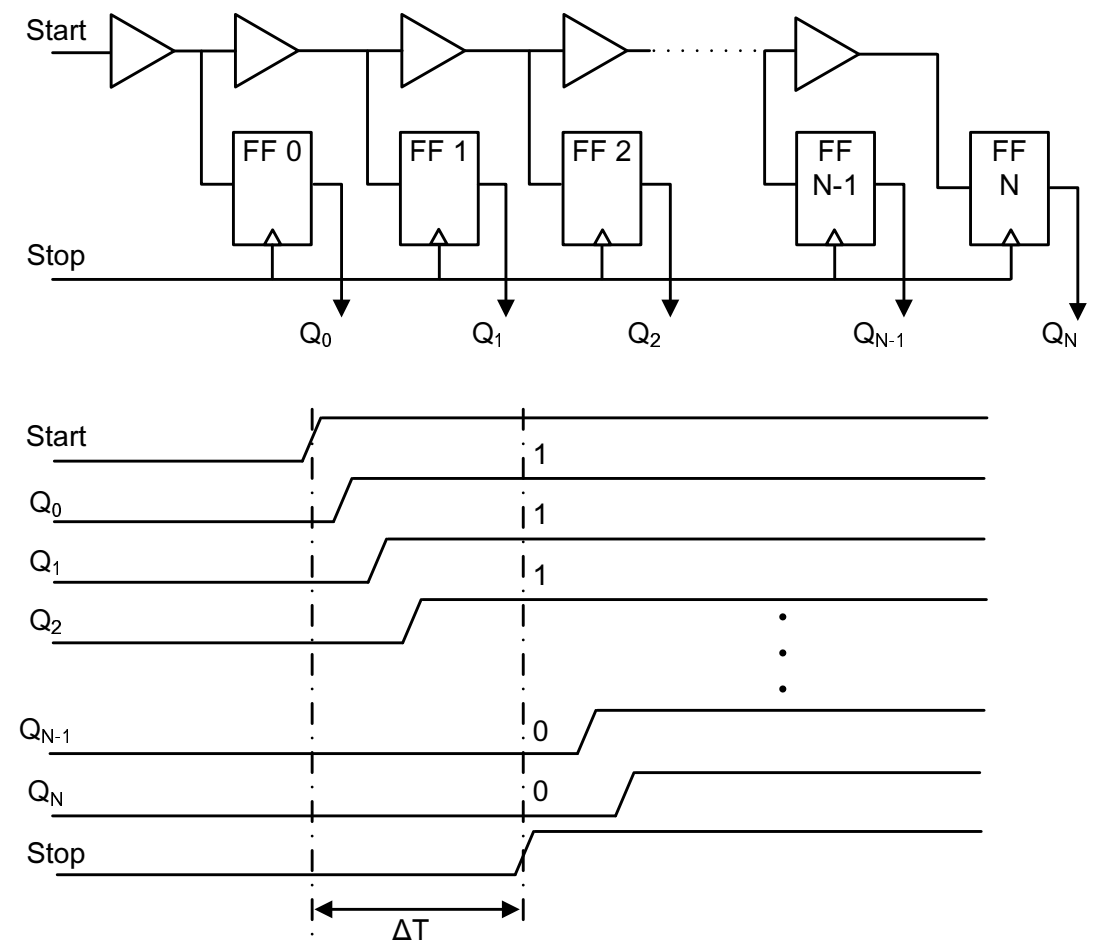

Figure 4.12: Scheme of the TDC and the operation principle.

Following the time-to-digital conversion, the output is immediately sent to the right shift which performs the divided by 2 function. The output of the shift and the data in Register2, which records the digital outcome of time delay in the current cycle, are added together to form a new time delay for another coming cycle. Since the input of the digital circuit such as adders and other blocks should 
be stable during the calculation phase-stage, two registers (Register 1 and Register 2) are thereby used for this design. A dual phase non-overlapping clock is constituted from $V_{H}$ and $V_{L}$ and it is synchronized by a clock generator. CLK1 and CLK2 ensure that the adder, the right shift and the updating of the time delay information to operate in a correct pace. It should be noted that the time interval between CLK2 and the new cycle should be sufficient for data processing and transfer. The timing diagram of the digital control is shown in Figure 4.13.

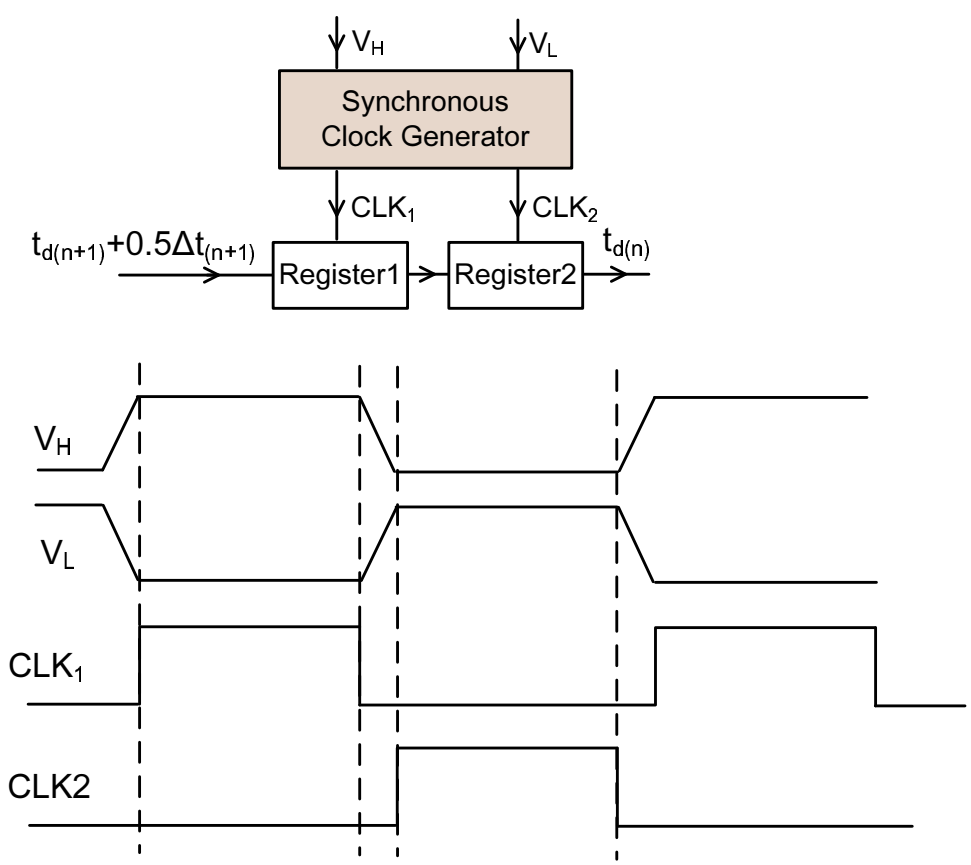

Figure 4.13: Timing diagram of the digital control.

A digital lock circuit is used to lock the time delay when the falling edge of the control signals $V_{G 1}\left(V_{G 2}\right)$ gets very close to the $V_{H}\left(V_{L}\right)$. This function is carried out by a multiplexer and two logic comparators. The lock operation occurs under two conditions. The first is that the adder reaches the upper limit of the setting value. At that time, the lock circuit pulls CLK1 and CLK2 to ground, to avoid data refresh. Second, the outcome of TDC reaches the bottom limit of the setting value, which to prevent the reverse leakage happen.

The output of the 6-bit digital delay signals control the delay circuit to adjust the time interval between $S_{1}\left(S_{2}\right)$ and $R_{1}\left(R_{2}\right)$. The delay circuit consists of a fixed delay line which is used to shorten the control time of the circuit and an 
adjustable delay which converts the digital signal to the time delay. The structure of the delay circuit is shown in the Figure 4.14.

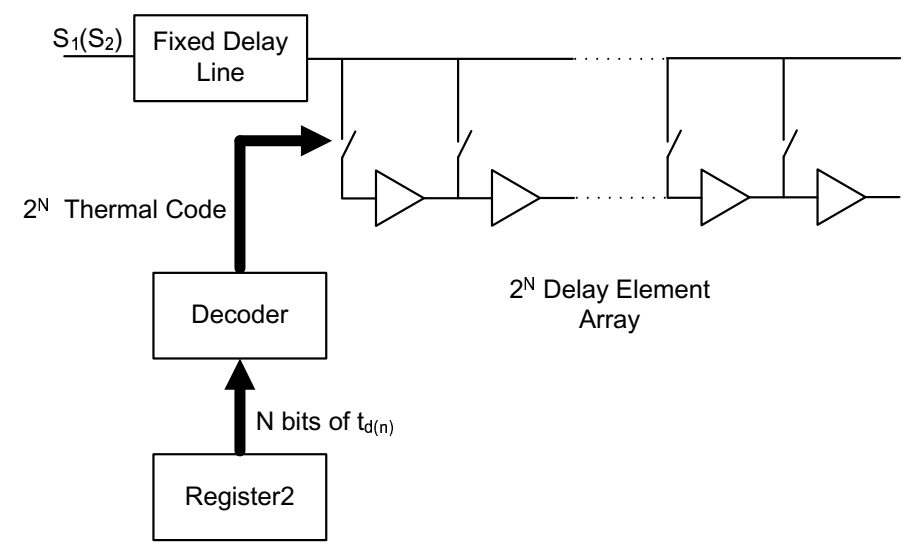

Figure 4.14: Schematic of the delay circuits.

\subsection{Experimental Results}

The proposed $15 \mathrm{~W}$ WPT active rectifier, not including the power stage, is fabricated with a $0.18 \mu \mathrm{m}$ high voltage CMOS process. The photo of the proposed die is depicted in Figure 4.15. The entire receiver board with the proposed rectifier and communication module, a full bridge Class-D power amplifier operating at ZVS (zero-voltage-switching) and a planar PCB coil pair are also shown in Figure 4.15. The specifications of the circuit elements are given in Table 4.1. The wireless power transfer system works with a transmission distance of $5 \mathrm{~cm}$.
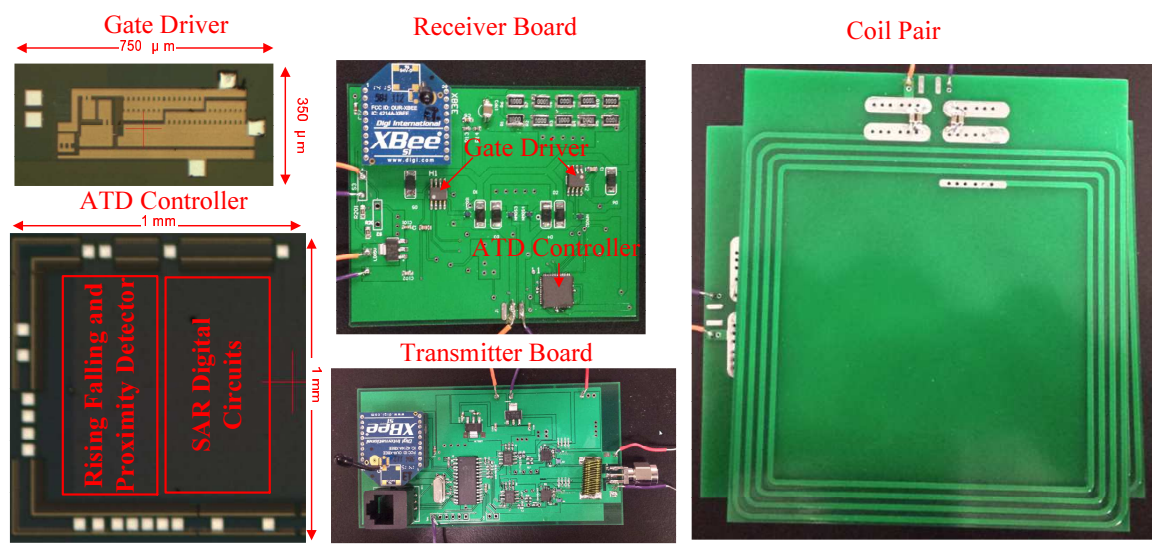

Figure 4.15: Die photo and whole rectifier with GaN FETs on PCB.

The operating frequency of the system is $6.78 \mathrm{MHz}$ and the output of the 
rectifier is adjusted to $5 \mathrm{~V}$ by the communication module. Figure 4.16 shows the measurement results of the proposed rectifier with ATD control. Figure 4.16 (a) shows that the $\mathrm{AC}$ input voltage (VH in Figure4.6) and the corresponding gate signal ( $V_{G 1}$ in Figure4.6) from fixed delay to locked steady-state, for an output at 5V/3A. The zoom-in wave form in Figure 4.16 (b) shows that the gate signal successfully shut down the power transistor exactly when VH fell below VREC. Similar waveform has been shown in Figure 4.16 (c) and (d) for an output of 5V/0.15 A. It can be seen for different output current, the duration time of the conduction of the GaN devices are different. But for both case, they have been shut down in time.

Table 4.1: Testing components of the proposed 6.78-MHz Rectifier

\begin{tabular}{|c|c|}
\hline Circuit Components & Value \\
\hline Power Transistors & EPC2014 \\
\hline Transmitting coil & $4.1 \mu \mathrm{H} / 0.43 \mathrm{ohms} @ 6.78 \mathrm{MHz}$ \\
\hline Receiving coil & $4.1 \mu \mathrm{H} / 0.43 \mathrm{ohms} @ 6.78 \mathrm{MHz}$ \\
\hline Compensation Capacitor & $100 \mathrm{pF} / 500 \mathrm{~V} / / 30 \mathrm{pF} / 500 \mathrm{~V} / / 5 \mathrm{pF} / 500 \mathrm{~V}$ \\
\hline Smoothing capacitor & $22 \mu \mathrm{F} / 50 \mathrm{~V}$ \\
\hline
\end{tabular}

To define the performance of rectifiers, power conversion efficiency (PCE) is often the most important index. It is defined as the ratio of Pout to Pin, where the former is the DC power consumed at $R_{L}$ while the latter is the $\mathrm{AC}$ power at the input to the rectifier. The $\mathrm{AC}$ power is defined as the average value of the product of $V_{A C}$ and $I_{A C}$ in Figure 4.6, given by

$$
P_{\text {in }}=\int_{0}^{t} V_{A C} I_{A C} d t
$$

while the output power is given by

$$
P_{\text {out }}=V_{\text {out }} \times I_{\text {out }}
$$

As a result, the efficiency of the rectifier is expressed as 

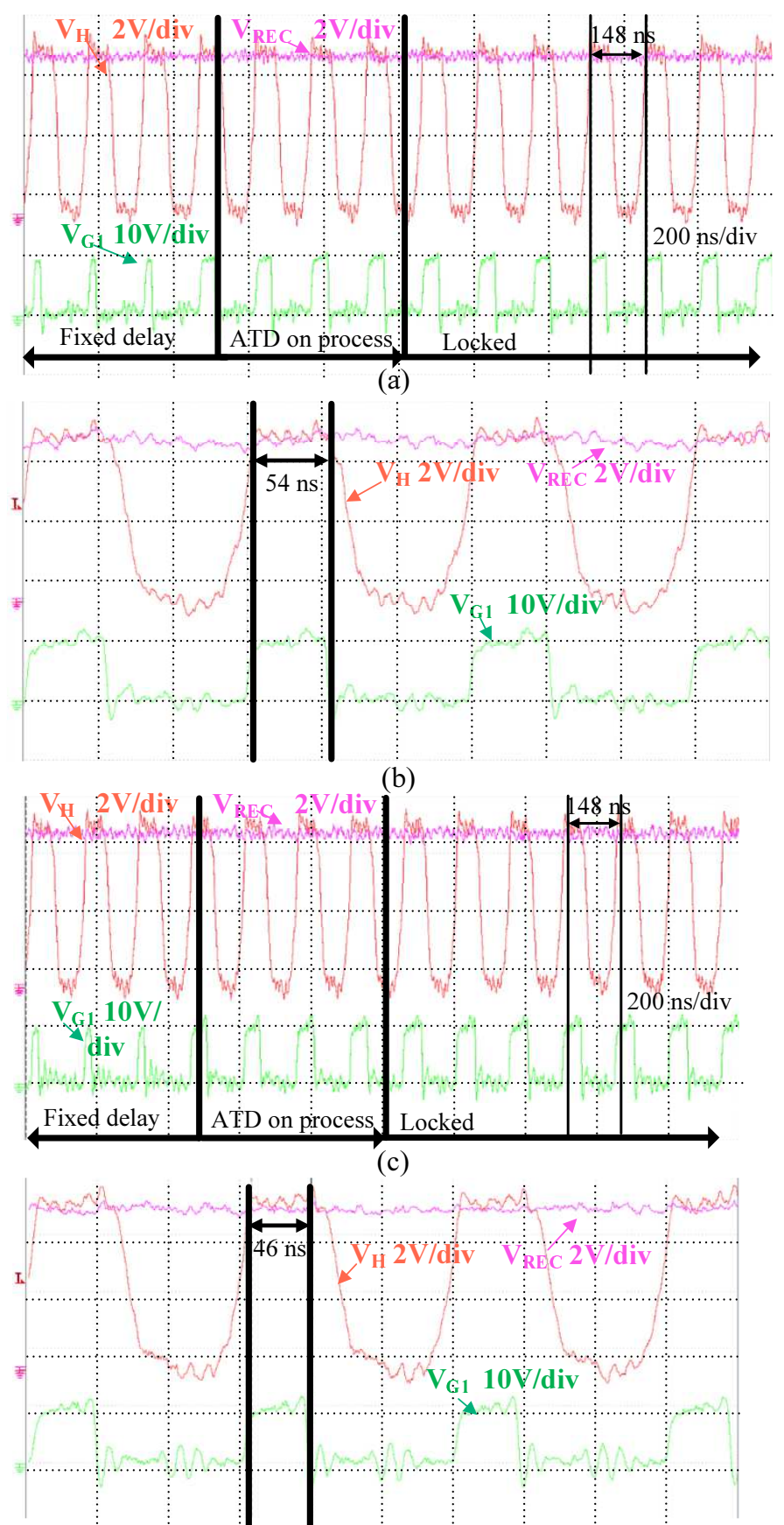

(d)

Figure 4.16: (a) Waveform of the active rectifier at 5V/3A. (b) Zoom-in view of (a).(c) Waveform of the active rectifier at 5V/0.15 A. (d) Zoom-in view of (c).

$$
\eta=\frac{P_{\text {out }}}{P_{\text {in }}}
$$

The test is conducted with the output current changing from $3 \mathrm{~A}$ to $0.15 \mathrm{~A}$ at a charging distance of $5 \mathrm{~cm}$. The measurement results shown in Figure 4.17 (a) 


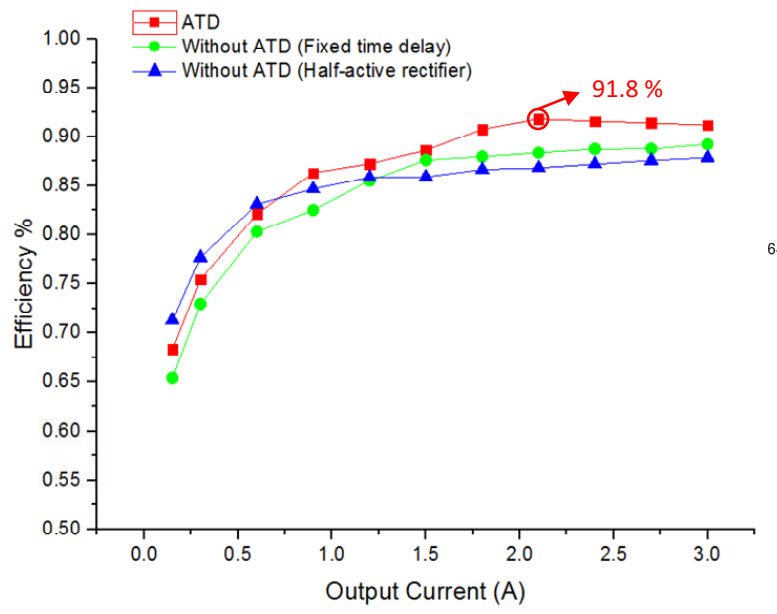

(a)

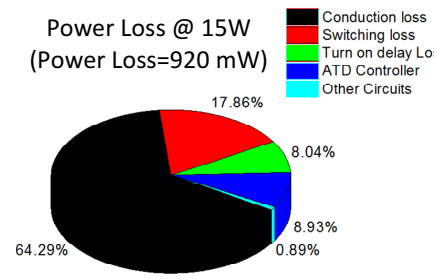

(b)

Power Loss @ 0.75W (Power Loss $=230 \mathrm{~mW}$ )

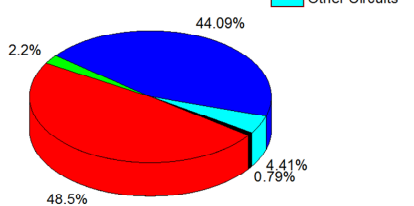

(c)

Figure 4.17: Measured efficiency of the rectifier.

indicates a $91.8 \%$ PCE at about 2 A. The efficiency of the rectifier with the fixed delay line and half-active rectifier are shown and compared in Figure 4.17 (a). At light load, the efficiency drops below 80\%. A pie chart in Figure 4.17 (b) and (c) shows the simulation result of power loss including sub-circuit loss, switching loss, and conduction loss. The turn on delay loss is the power loss due to the delay of the rising detector. Since the power consumption of the ATD controller is a static loss source and switching loss is due to the capacitor and gate voltage, both of which are almost unchanged at different power level. Such power loss contributes close to $93 \%$ of the total power loss in light load mode and $25 \%$ of the total power loss in heavy load mode. This explains the reason of the efficiency degradation of the rectifier in the light load condition. A solution for this problem is to change the rectifier to half-active mode at the light load.

Table 4.2 summarizes the performance comparison with state-of-the-art rectifiers for WPT megahertz applications [49], [48] and [41]. This single-stage regulator provides the highest output power and comparable receiver efficiency is better than most of other works. 
Table 4.2: Performance comparison with the proposed rectifier

\begin{tabular}{|c|c|c|c|c|c|c|}
\hline Structure & $\begin{array}{c}\text { Half } \\
\text { Active }\end{array}$ & $\begin{array}{c}\text { Active } \\
\text { diode }\end{array}$ & $\begin{array}{c}\text { Active } \\
\text { diode } \\
\text { with RCC }\end{array}$ & $\begin{array}{c}\text { Un } \\
\text {-bal. } \\
\text { comp. }\end{array}$ & $\begin{array}{c}\text { DLL } \\
\text { control }\end{array}$ & $\begin{array}{c}\text { ATD } \\
\text { control }\end{array}$ \\
\hline $\begin{array}{c}V_{\text {out }} \\
(\mathrm{V})\end{array}$ & 5.83 & 3 & 3.5 & 1 & 8.6 & 5 \\
\hline $\begin{array}{c}P_{\text {out }} \\
\text { Max (mW) }\end{array}$ & 11 & 5 & 6.8 & 5 & 6000 & 15000 \\
\hline $\begin{array}{c}\text { Resonant } \\
\text { f. (MHz) }\end{array}$ & 4 & 5 & 13.56 & 1.5 & 6.78 & 6.78 \\
\hline Peak eff. & N.A. & $80 \%$ & $87 \%$ & $87.5 \%$ & $91.5 \%$ & $91.8 \%$ \\
\hline Process & $0.35 \mu \mathrm{m}$ & $0.35 \mu \mathrm{m}$ & $\begin{array}{c}\mathrm{CMOS} \\
0.35 \mu \mathrm{m}\end{array}$ & $\begin{array}{c}\mathrm{CMOS} \\
0.35 \mu \mathrm{m}\end{array}$ & $\begin{array}{c}\mathrm{BCD} \\
0.35 \mu \mathrm{m}\end{array}$ & $\begin{array}{c}\mathrm{CMOS} \\
0.18 \mu \mathrm{m} \\
(\text { off-chip } \\
\text { GaN })\end{array}$ \\
\hline
\end{tabular}

\subsection{Conclusions}

In this chapter, a full-active rectifier with adaptive delay control (ATD) for 6.78 $\mathrm{MHz}$ wireless power transfer application is presented. This rectifier has a high power conversion efficiency because the proposed control algorithm not only eliminate the leakage current but also maximize the conduction of the active switch. This chip was implemented using $0.18 \mu \mathrm{m}$ AMS technology with four GaN transistors being used for the power stage of the rectifier. The maximum efficiency of the proposed rectifier is highly competitive at $90.6 \%$ under output DC voltage condition of $5 \mathrm{~V}$ while providing $15 \mathrm{~W}$ output power, which is the highest among relevant works reported to-date. 


\section{Chapter 5}

\section{Analysis and Hybrid Control}

\section{Method for 6.78 MHZ Wireless}

\section{Power Transfer System with}

\section{Varying Transmission Distance}

\subsection{Introduction}

Although magnetic resonant WPT can improve the performance of the system for centimetres application, one problem is that as the transmission distance increases to a certain value, the power transfer capability decreases dramatically, known as frequency splitting [109], [51], [52] and [110]. As a result, the system is not able to provide enough output power to the load. To overcome the frequency splitting problem, a combined control method of frequency tuning and phase shift tuning is proposed in this chapter. This control method tunes the operating frequency of the magnetic resonate WPT system for short power transfer distance while tunes the phase shift for large power transfer distance. Meanwhile, the control is able to keep the overall efficiency at a high level in a wide range of power transfer distance and load condition. The rest of this chapter is organized as follows. Section 5.2 describes detailed analysis of the frequency splitting phe- 
nomena based on a basic topology of a magnetic resonant WPT system and the equivalent circuit model. Equations of power transfer efficiency, output power, and frequency splitting have been derived. Based on the theoretical analysis, the control flow of the system and the parameter optimization of the system are described in Section 5.3. Experimental results and conclusions are presented in Sections 5.4 and 5.5, respectively.

\subsection{Analysis of Magnetic Resonant Wireless Power Transfer System}

\subsubsection{System Overview}

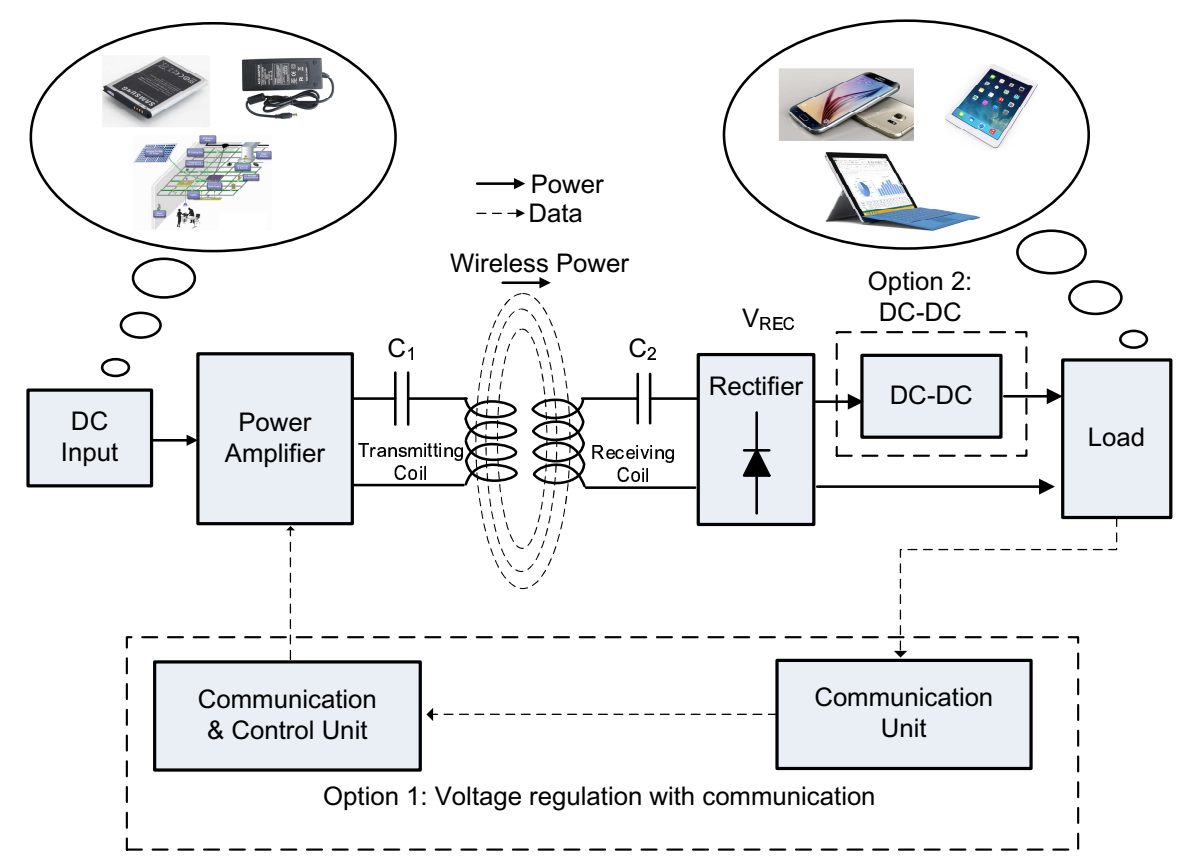

Figure 5.1: Wireless power transfer (WPT) system.

The WPT system is redrawn in Figure 5.1. The power is transferred wirelessly from the transmitting coil to the receiving coil. Both coils have been compensated to enhance the system efficiency and power transfer capability required for different applications. In the transmitter, the power amplifier (PA) transforms the input $\mathrm{DC}$ voltage to a high frequency $\mathrm{AC}$ voltage that drives the transmitting coil to generate $\mathrm{AC}$ magnetic fluxes. In the receiver, the coupled AC voltage is 
rectified into a DC voltage $\left(V_{R E C}\right)$ by a rectifier. An additional DC-DC block and LDO is added to regulate the output voltage at a constant value. Wireless communication has always been utilized between the transmitter and the receiver to control the power flow of the system and to optimize the overall efficiency.

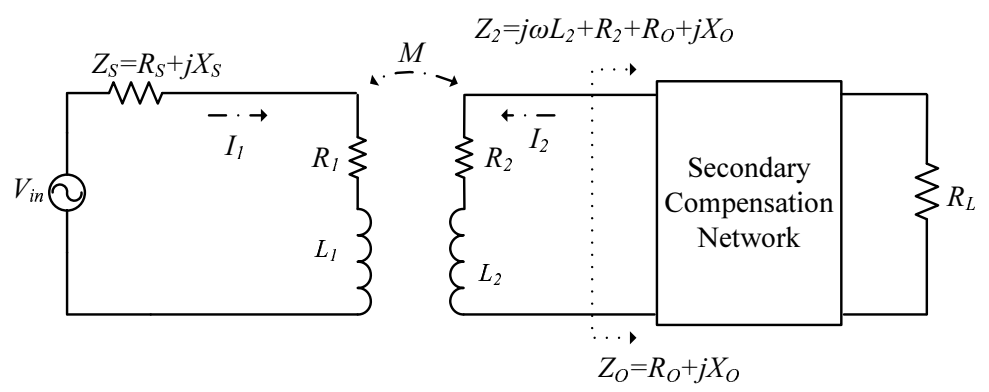

(a)

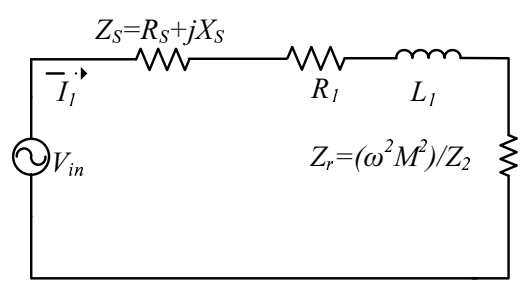

(b)

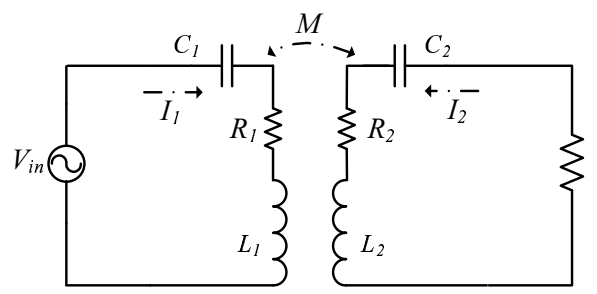

(c)

Figure 5.2: Simplified WPT circuit model: (a) Topology, (b) RLT model, (c) S-S circuit topology.

The WPT system can be simplified to an equivalent circuit model as shown in Figure 5.2.(a). $L_{1}$ and $L_{2}$ are the self-inductance of the transmitting and receiving coils, respectively. The interaction between the two coils induces an effect of mutual inductance, M. Each coil has an equivalent series resistance identified as $R_{1}$ and $R_{2}$. The transmitter including the DC input, DC-AC converter and the compensation network is replaced by an $\mathrm{AC}$ voltage source $V_{\text {in }}$ and an equivalent source series impedance $Z_{s}$, which is equal to $R_{s}+\mathrm{j} X_{s}$. Since the source impedance is usually very negligibly small, it is omitted in the following analysis. As for the receiver, the compensation network, rectifier and DC-DC converter are replaced by an equivalent load impedance $Z_{o}$, which is equal to $R_{L}+\mathrm{j} X_{o}$. The loop impedance of the receiver $Z_{2}$ is given by:

$$
Z_{2}=j\left(\omega L_{2}+X_{O}\right)+R_{2}+R_{L}=j X_{2}+R_{2}+R_{L}
$$


Based on the simplified model and Kirchhoffs voltage law (KVL), the equations for the voltage -current relationships of the WPT system are derived as

$$
\left\{\begin{array}{l}
V_{\text {in }}=I_{1}\left(j \omega L_{1}+Z_{S}+R_{1}\right)+I_{2} j \omega M \\
0=I_{2} Z_{2}+I_{1} j \omega M
\end{array}\right.
$$

where $\mathrm{M}$ is the mutual inductance

$$
M=k \sqrt{L_{1} L_{2}}
$$

in Equation (5.3), $\mathrm{k}$ is the coupling coefficient between the two coils. According to Equqtion (5.1), (5.2) and the reflected load theory (RLT) ( [14], [21], and [111]), the entire receiver can be replaced by a reflected impedance $Z_{r}$, and be integrated into the transmitter, as shown in Figure 5.2(b). $Z_{r}$ is expressed as

$$
Z_{r}=\frac{\omega^{2} M^{2}}{Z_{2}}
$$

Considering only the active power of the system, the efficiency is the ratio of the active power dissipated by $Z_{L}$ to the active power developed by $V_{i n}$. The overall efficiency can be expressed as the product of the efficiency of the transmitter and the receiver, which is given by,

$$
\left\{\begin{array}{l}
\eta_{1}=\frac{\Re\left(Z_{r}\right)}{\Re\left(Z_{r}\right)+R_{1}+R_{S}} \\
\eta_{2}=\frac{R_{L}}{R_{L}+R_{2}}
\end{array}\right.
$$

According to Equation (5.5), the system efficiency is derived as

$$
\eta=\eta_{1} \eta_{2}=\frac{\omega^{2} M^{2} R_{L}}{\omega^{2} M^{2}\left(R_{L}+R_{2}\right)+\left(R_{1}+R_{S}\right)\left[\left(R_{L}+R_{2}\right)^{2}+X_{2}^{2}\right]}
$$

From Equation (5.6), it can be seen that the efficiency will be improved if the receiver is compensated to be resistive, thereby nullifying $X_{2}$. For this reason, 
series compensation technique is used for the receiver since a series resonant LC network can achieve a zero $X_{2}$. The transmitter is also compensated in series to give a unity power factor (UPF), thus enhancing the power transfer capability. An equivalent circuit representation of the series-series (S-S) model is shown in the Figure 5.2 (c). Meanwhile, the $R_{S}$ has been removed for simplicity, since it is substantially small when compared to $R_{1}$. The analyses presented in the subsequent sections of this thesis are based on this model thereafter.

\subsubsection{Maximum Efficiency of a Series-Series (S-S) Compensation}

A lot of research has analysed the maximum power transfer efficiency ( [112], [113], [114], [115], [116], [117] , [118], [119], [120], [91], and [121]). In an S-S compensation WPT system as shown in Figure 5.2 (c), assuming that the power losses of inverter and ferrite shielding is omitted, the KVL state equations can be written as

$$
\left\{\begin{array}{l}
V_{i n}=I_{1}\left(R_{1}+j \omega L_{1}+\frac{1}{j \omega C_{1}}\right)+I_{2} j \omega M \\
0=I_{2}\left(R_{2}+R_{L}+j \omega L_{2}+\frac{1}{j \omega C_{2}}\right)+I_{1} j \omega M
\end{array}\right.
$$

The $Z_{2}, Z_{r}$ can be derived as

$$
\begin{gathered}
Z_{2}=R_{2}+R_{L}+j\left(\omega L_{2}-\frac{1}{\omega C_{2}}\right) \\
Z_{r}=\frac{\omega^{2} M^{2}}{R_{2}+R_{L}+j\left(\omega L_{2}-\frac{1}{\omega C_{2}}\right)}
\end{gathered}
$$

Then the efficiency of the system is computed as

$$
\eta=\frac{\omega^{2} M^{2} R_{L}}{\omega^{2} M^{2}\left(R_{L}+R_{2}\right)+R_{1}\left(R_{L}+R_{2}\right)^{2}}
$$

if the system works in the resonant condition which means 


$$
\omega=\omega_{0}=\frac{1}{\sqrt{L_{1} C_{1}}}=\frac{1}{\sqrt{L_{2} C_{2}}}
$$

where $\omega$ is operating frequency and $\omega_{0}$ is the resonant frequency. The equation can be further derived that

$$
\begin{aligned}
& \eta=\frac{\omega^{2} M^{2}}{\omega^{2} M^{2}+2 R_{1} R_{2}+R_{1} R_{L}+\frac{\omega^{2} M^{2} R_{2}+R_{1} R_{2}{ }^{2}}{R_{L}}} \\
& \geq \frac{\omega^{2} M^{2}}{\omega^{2} M^{2}+2 R_{1} R_{2}+2 \sqrt{R_{1} R_{L} \times \frac{\omega^{2} M^{2} R_{2}+R_{1} R_{2} 2}{R_{L}}}} \\
& =\frac{\omega^{2} M^{2}}{\omega^{2} M^{2}+2 R_{1} R_{2}+2 \sqrt{R_{1}\left(\omega^{2} M^{2} R_{2}+R_{1} R_{2}{ }^{2}\right)}}
\end{aligned}
$$

when

$$
R_{L}=R_{O P T}=\sqrt{\frac{\omega^{2} M^{2} R_{2}+R_{1} R_{2}^{2}}{R_{1}}}=\sqrt{\frac{\omega^{2} k^{2} L_{1} L_{2} R_{2}+R_{1} R_{2}^{2}}{R_{1}}}
$$

Equations (5.12) and (5.13) imply that the optimized load value is related to the coupling coefficient which is proportional to the distance between the transmitter and receiver. Figure 5.3 shows the system efficiency over a wide range of load characteristics. It can be concluded that, for strong coupling $(\mathrm{k}=0.5)$, the efficiency is not much affected by the load conditions, while for loose coupling $(k=0.07)$, the efficiency drops drastically as the load deviates greatly from the optimum value. Thus, efforts should be elicited in optimizing the load for the case of loose coupling.

\subsubsection{Frequency Splitting Analysis}

Another important design specification of the magnetic resonant WPT system is the output power. The output power is defined as the level of real power that can be transferred to the load in the WPT system. In extreme strongly coupled conditions $(\mathrm{k}>0.9)$, the output power is a function of the ratio of the number of turns of the transmitting coil to the number of turns of the receiving coil. In a 


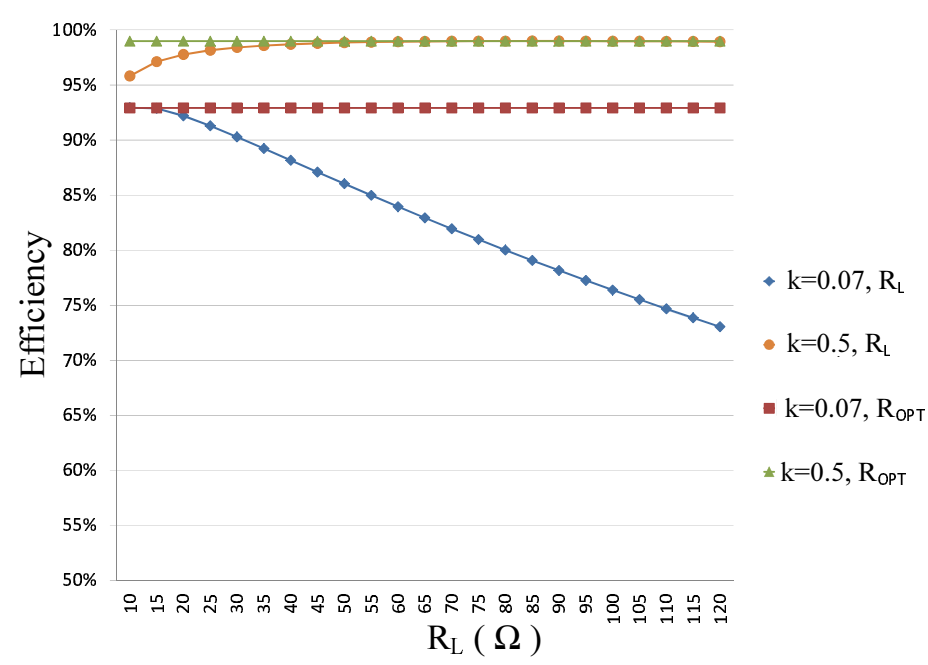

Figure 5.3: Efficiency with load variation.

loosely coupled S-S WPT system, the output power is affected by the coupling coefficient, the value of the capacitor and the operating frequency( [109], [51], [52], [110], [122] and [123]). For this reason, a detailed analysis on the maximum output power conditions and frequency splitting is carried out for a varying range of coupling coefficients.

From Figure 5.2, the output power of the system can be calculated as

$$
P_{\text {out }}=I_{2}^{2} R_{L}=\left|\frac{j \omega M I_{1}}{Z_{2}}\right|^{2} R_{L}
$$

where $I_{1}$ is the transmitting coil current, which can be calculated by the RLT based on the Figure 5.2 (b) as

$$
I_{1}=\frac{V_{\text {in }}}{j\left(\omega L_{1}-\frac{1}{\omega C_{1}}\right)+R_{1}+\frac{\omega^{2} M^{2}}{j\left(\omega_{2} L_{2}-\frac{1}{\omega_{2} C_{2}}\right)+R_{2}+R_{L}}}
$$

For simplification, the efficiency of the overall system can be expanded as

$$
P_{\text {out }}=\frac{V_{\text {in }}^{2} C_{1}^{2} C_{2}^{2} M^{2} R_{L}}{F}
$$

where

$$
F=A \omega^{2}+B+C \frac{1}{\omega^{2}}+D \frac{1}{\omega^{4}}+\frac{1}{\omega^{6}}
$$


which is expressed in terms of parameters A, B, C, and D:

$$
A=M^{2} C_{1}^{2} C_{2}^{2}-2 M^{2} C_{1}^{2} C_{2}^{2} L_{1} L_{2}+C_{1}^{2} C_{2}^{2} L_{1}^{2} L_{2}^{2}
$$

$$
\begin{gathered}
B=2 M^{2} C_{1}^{2} C_{2}^{2} R_{1} R_{2}+2 M^{2} C_{1}^{2} C_{2}^{2} R_{1} R_{L}+C_{1}^{2} C_{2}^{2} L_{1}^{2} R_{2}^{2}+2 C_{1}^{2} C_{2}^{2} L_{1}^{2} R_{2} R_{L}+C_{1}^{2} C_{2}^{2} L_{2}^{2} R_{L}^{2} \\
+C_{1}^{2} C_{2}^{2} L_{2}^{2} R_{1}^{2}+2 M^{2} C_{1}^{2} C_{2} L_{1}+2 M^{2} C_{1} C_{2}^{2} L_{2}-2 C_{1}^{2} C_{2} L_{1}^{2} L_{2}-2 C_{1} C_{2}^{2} L_{1} L_{2}^{2} \\
\doteq 2 M^{2} C_{1}^{2} C_{2} L_{1}+2 M^{2} C_{1} C_{2}^{2} L_{2}-2 C_{1}^{2} C_{2} L_{1}^{2} L_{2}-2 C_{1} C_{2}^{2} L_{1} L_{2}^{2}
\end{gathered}
$$

$$
\begin{gathered}
C=C_{1}^{2} C_{2}^{2} R_{1}^{2} R_{2}^{2}+2 C_{1}^{2} C_{2}^{2} R_{1}^{2} R_{2} R_{L}+C_{1}^{2} C_{2}^{2} R_{1}^{2} R_{L}^{2}-2 C_{1}^{2} C_{2} L_{2} R_{1}^{2}-2 C_{1} C_{2}^{2} L_{1} R_{2}^{2} \\
-4 C_{1} C_{2}^{2} L_{1} R_{2} R_{L}-2 C_{1} C_{2}^{2} L_{2} R_{L}^{2}-2 M^{2} C_{1} C_{2}+C_{1}^{2} L_{1}^{2}+4 C_{1} C_{2} L_{1} L_{2}+C_{2}^{2} L_{2}^{2} \\
\doteq-2 M^{2} C_{1} C_{2}+C_{1}^{2} L_{1}^{2}+4 C_{1} C_{2} L_{1} L_{2}+C_{2}^{2} L_{2}^{2}
\end{gathered}
$$

$$
D=C_{1}^{2} R_{1}^{2}+C_{2}^{2} R_{2}^{2}+2 C_{2}^{2} R_{2} R_{L}+C_{2}^{2} R_{L}^{2}-2 C_{1} L_{1}-2 C_{2} L_{2} \doteq-2 C_{1} L_{2}-2 C_{2} L_{2}
$$

Replacing the $L_{1}, L_{2}, C_{1}$ and $C_{2}$ with Equations (5.3) and (5.11), it can be shown that

$$
F=\left(\frac{k^{2}}{\omega_{o}^{8}}-\frac{2 k^{2}}{\omega_{o}^{8}}+\frac{1}{\omega_{o}^{8}}\right) \omega^{2}+\left(\frac{6}{\omega_{o}^{2}}-\frac{2 k^{2}}{\omega_{o}^{2}}\right) \frac{1}{\omega^{2}}-\frac{4}{\omega_{o}^{2}} \frac{1}{\omega^{4}}+\frac{1}{\omega^{6}}
$$

By differentiating Equation 5.22 with respect to $\omega^{2}$ and calculating the roots of the first-order equation, given by

$$
\frac{\partial F}{\partial \omega^{2}}=0
$$

it can be found that the valid roots of Equation (5.23) are: 


$$
\begin{gathered}
\omega_{1}{ }^{2}=\frac{\omega_{0}{ }^{2}}{1+k} \\
\omega_{2}^{2}=\frac{\omega_{0}^{2}}{1-k} \\
\omega_{3}^{2}=\frac{\left(\sqrt{4-3 k^{2}}-1\right) \omega_{o}^{2}}{1-k^{2}}
\end{gathered}
$$

Substituting Equations (5.24), (5.25) and (5.26) into the second derivative of Equation (5.22), it can be determined whether $\omega_{1} \omega_{2}$, and $\omega_{3}$ are local maximum or minimum:

$$
\begin{gathered}
\left.\frac{\partial^{2} F}{\partial\left(\omega^{2}\right)^{2}}\right|_{\left(\omega^{2}=\omega_{1}^{2}\right)}=\frac{8(1+k)^{2} k^{2}}{\omega_{0}^{10}}>0 \\
\left.\frac{\partial^{2} F}{\partial\left(\omega^{2}\right)^{2}}\right|_{\left(\omega^{2}=\omega_{2}^{2}\right)}=\frac{8(1-k)^{2} k^{2}}{\omega_{0}^{2}}>0 \\
\left.\frac{\partial^{2} F}{\partial\left(\omega^{2}\right)^{2}}\right|_{\left(\omega^{2}=\omega_{3}^{2}\right)}=\frac{8(1-k)^{3}(1+k)^{3} K(K-2)}{3(K-1)^{3} \omega_{0} 10}<0
\end{gathered}
$$

where

$$
K=\sqrt{4-3 k^{2}}
$$

It is deduced that $\mathrm{F}$ has local minimum value at $\omega_{1}$ and $\omega_{2}$, and local maximum value at $\omega_{3}$. From Equations (5.24) to (5.26) it is clear that the when the coupling is strong, $\omega_{1}, \omega_{2}$ and $\omega_{3}$ are far away from each other. This is known as frequency splitting phenomena. The splitting coefficient is defined as

$$
d_{\omega}=\left(\frac{1}{\sqrt{1-k}}-\frac{1}{\sqrt{1+k}}\right) \omega_{0}
$$

Equation (5.31) determines the splitting frequency of the two peaks of the output power. Figure 5.4 depicts a 2-D plot of calculated data of output power versus 
operating frequency, which demonstrates the frequency splitting occurrence. The picture includes five curves for different coupling coefficients. It can be seen that when the coupling coefficient is below $0.07, \omega_{1}, \omega_{2}$ and $\omega_{3}$ converge. To verify the analysis, the comparison of calculations results and experimental results $(\mathrm{k}=0.5, \mathrm{k}=0.07)$ are shown in Figure 5.5. The parameters used for Figure5.4 are shown in Table 5.1.

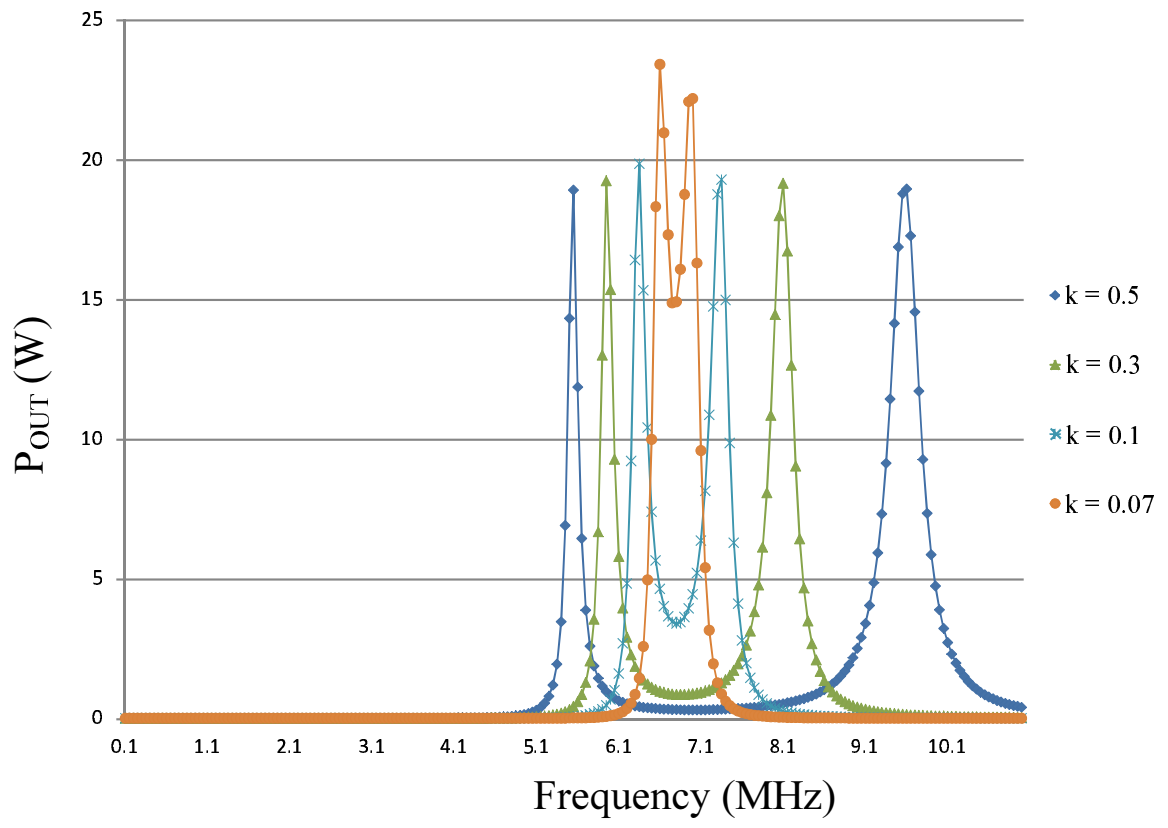

Figure 5.4: Frequency splitting effect (Calculstion results).

Table 5.1: Parameters in Figure 5.4

\begin{tabular}{|c|c|c|}
\hline Symbol & Parameters & Value \\
\hline$f_{0}$ & Resonance frequency & $6.78 \mathrm{MHz}$ \\
\hline$L_{1}$ and $L_{2}$ & Coil inductance & $4 \mu \mathrm{H}$ \\
\hline$C_{1}$ and $C_{2}$ & Compensation Capacitor & $138 \mathrm{pF}$ \\
\hline$R_{1}$ and $R_{2}$ & ESR of coils @ $f_{0}$ & $0.43 \Omega$ \\
\hline$R_{L}$ & Load resistance & $10 \Omega$ \\
\hline
\end{tabular}

Another interesting phenomenon is that, at frequencies $\omega_{1}, \omega_{2}$ and $\omega_{3}$, the phase angles of the input impedance $V_{\text {in }}$ (as seen from the AC source), which is given by

$$
Z_{\text {in }}=Z_{r}+R_{1}+j\left(\omega L_{1}-\frac{1}{\omega C_{1}}\right)
$$




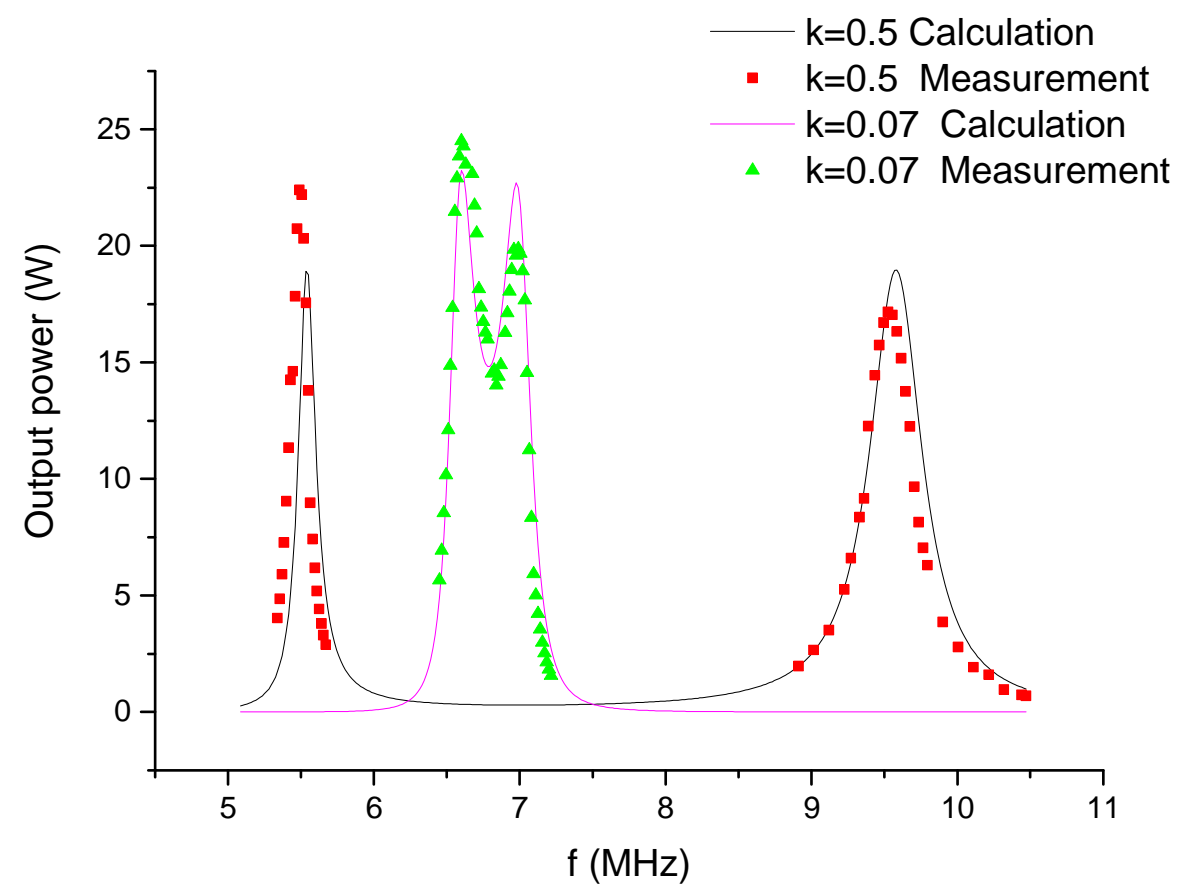

Figure 5.5: Frequency splitting effect (Calculstion and experimental results).

are zero. To approve that, Equation (5.32) can be expanded as

$$
Z_{\text {in }}=\frac{\omega^{2} M^{2}\left(R_{2}+R_{L}\right)}{\left(R_{2}+R_{L}\right)^{2}+\left(\omega L_{2}-\frac{1}{\omega C_{2}}\right)^{2}}+R_{1}+j\left(\omega L_{1}-\frac{1}{\omega C_{1}}-\frac{\omega^{2} M^{2}\left(\omega L_{1}-\frac{1}{\omega C_{1}}\right)}{\left(R_{2}+R_{L}\right)^{2}+\left(\omega L_{1}-\frac{1}{\omega C_{1}}\right)^{2}}\right)
$$

Using Equation (5.11), and assuming that the two coils are identical, the imaginary part of $Z_{\text {in }}$ is normalized and simplified as

$$
\mathfrak{I}\left(Z_{\text {in }}\right)=\frac{\left(\omega_{0}+\omega\right)\left(\omega_{0}-\omega\right)\left(k \omega^{2}-\omega^{2}+\omega_{0}^{2}\right)\left(k \omega^{2}+\omega^{2}-\omega_{0}^{2}\right)}{\omega_{0}^{2} C_{1} \omega E}
$$

where

$$
E=\omega^{2} C_{1}{ }^{2} R_{2}{ }^{2} \omega_{0}{ }^{4}+2 \omega^{2} C_{1}{ }^{2} R_{2}{ }^{2} R_{L}{ }^{2} \omega_{0}{ }^{4}+\omega^{2} C_{1}{ }^{2} R_{L}{ }^{2} \omega_{0}{ }^{4}+\omega^{4}+\omega_{0}^{4}-2 \omega^{2} \omega_{0}^{2}
$$

From Equation (5.34), it is clear that the phase angle is zero at $\omega_{1}, \omega_{2}$ and $\omega_{0}$. 


\subsection{Voltage Control Method and Efficiency Optimization}

\subsubsection{Voltage Control Method Description}

Effective control of output voltage is one of the critical criteria for achieving optimal wireless power transfer for portable consumer electronics. The regulation approach can be placed in either the transmitter or the receiver, depending on the requirements of different applications, like the area of the device, output voltage tolerance and response time ( [124], [125], [126], [127] and [128], ).

According to the previous analysis, the system always has peak efficiency at resonant frequency. So it is important to optimize the system efficiency as the output voltage requirement is met ( [129], [130], [131], [132], [133], [121] and [134]). For loose coupling condition, the output power is enough to provide the standard voltage. For strong coupling condition, unfortunately, the output voltage is not enough, as shown in Figure $5.4(\mathrm{k}=0.3-0.5)$.

According to the circuit analysis presented in section 5.2, the system will always operate at its peak efficiency at the resonant frequency. For loose coupling conditions, the output power is adequate to provide the required standard voltage. For strong coupling conditions, unfortunately, the output power is far from being practical, as shown in Figure 5.4 for $\mathrm{k}$ varies from 0.3 to 0.5 .

This implies that it is possible to use phase shift tuning to control the power flow for weakly coupled cases whereas the operating frequency must be tuned to meet the output power for strongly coupled cases. Since the equivalent series resistance (ESR) of the coil is proportional to the frequency, the operating frequency will be tuned towards $\omega_{1}$ instead of $\omega_{2}$.

The detailed voltage control flow chart of the proposed control methodology is shown in Figure 5.6. Its objective is to guarantee that the power transferred to the load is adequate to provide the standard voltage for general-purpose applications.

To start with, an initial $\mathrm{AC}$ input voltage $V_{\text {in }}$ is applied to the transmitter. The 


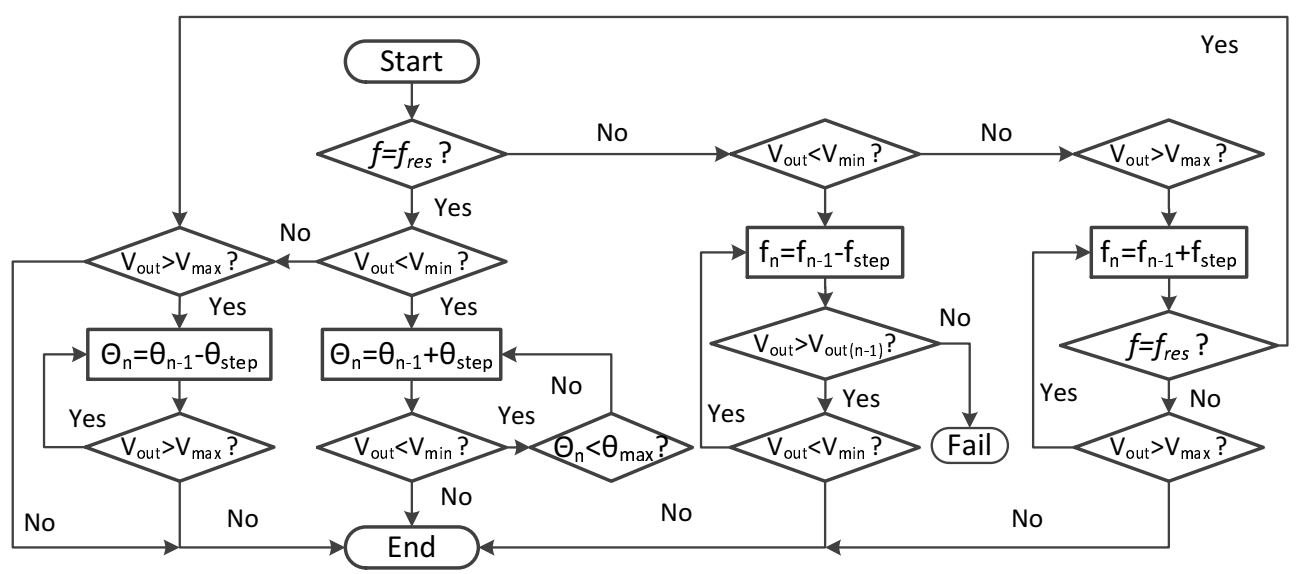

Figure 5.6: System control flow.

frequency of input voltage equals the resonant frequency $(6.78 \mathrm{MHz})$ and the phase shift of the amplifier is fixed at $10 \%$.

After initiating the power transfer process, the receiver begins to transfer the voltage error packets back to the transmitter and the micro-controller begins the phase shift tuning process according to the error packets. If the output voltage is able to meet the minimum value, the control is considered successful. Otherwise, if the output voltage is still lower than the minimum required output voltage when the phase shift is tuned to $\theta_{\max }$, the phase shift control is considered not meeting the requirement.

If the phase shift test fails in this way, frequency tuning begins. The controller tunes the frequency towards $\omega_{1}$. We can in fact construe from Figure 5.4, that it is also possible to tune the frequency towards $\omega_{2}$. However, it is imperative to take note that the ESR of the coil increases with increased frequency, hence a lower frequency is preferred( [135], [136], [137], [138] and [139]). Once the output voltage is larger than the minimum value, the frequency tuning process ends. If the load or the transfer distances change during power transfer, the process continues according to one of following scenarios:

Scenario 1: This is pursued provided the operating frequency is different from the resonant frequency. If the output voltage is lower than the required voltage $\left(V_{\min }\right)$, the frequency will be set lower to provide sufficient voltage. If the $V_{\min }$ 
requirement is still not met despite the frequency adjusted lower than $\omega_{1}$, the control is considered as failed. Conversely, if $V_{\text {out }}$ is higher than $V_{\text {max }}$, the frequency will be tuned higher. If the frequency is tuned to resonant frequency but Vout is still higher than $V_{\max }$, the phase is tuned lower to match the requirement.

Scenario 2: This happens when the operating frequency equals the resonant frequency. If the output voltage is larger than $V_{\max }$, the phase is tuned lower to meet the requirement. If the output voltage is lower than $V_{\min }$, the phase is tuned higher. If, when the phase is tuned to $\theta_{\max }$, it still cannot provide $V_{\min }$, the frequency control starts as described in Scenario 1

It is worth noting that in real-life application, the signal of output voltage would be kept being sent back to the transmitter with a communication ZigBee module. Unlike the single-stage voltage regulator mentioned in the Chapter 3, the voltage control proposed in this chapter is used to extend the power transmission distance over a wide range. Such applications target to high end products so the requirement of the cost is not as stringent.

\subsubsection{Parameter Design for Power Transfer Efficiency}

From Figure 5.4, it can be seen that the output power would be very low in short distance $(\mathrm{k}=0.5)$. So in this case, the frequency is supposed to be tuned lower to meet the power requirement. However, due to the analysis mentioned before, the efficiency would decrease when the system works under non-resonant condition. The efficiency deterioration factor, Dete, is defined as:

$$
\text { Dete }=\frac{1}{\eta_{(\omega)}}
$$

The deterioration factor at 1 and 2 can be calculated as

$$
\left\{\begin{array}{l}
\operatorname{Dete}_{(\omega 1)}=\frac{1}{\eta_{(\omega 1)}}-\frac{1}{\eta_{(\omega 0)}}=\frac{R}{R_{L}} \\
\operatorname{Dete}_{(\omega 2)}=\frac{1}{\eta_{(\omega 2)}}-\frac{1}{\eta_{(\omega 0)}}=\frac{R}{R_{L}}
\end{array}\right.
$$


It can be seen that Dete is related to the ratio of ESR of the coil to load resistance. The worst-case scenario happens when the system works under full load condition, at which the efficiency would drop around 4\%. In practical applications, this drop could be less because: 1) frequency tuning towards $\omega_{1}$ means a smaller ESR due to lower frequency; 2) the operating frequency does not have to be equal to $\omega_{1}$, but low enough to provide adequate required voltage. Besides, frequency splitting happens when $\mathrm{k}$ is large, which further increases the efficiency due to Equation 5.6. This approach is adopted in the proposed control method presented in the subsequent section. The theoretical and experimental data of efficiency vs. $\mathrm{f}$ is given in Figure 5.5. This analysis validates that the system being able to work with high efficiency and output power in both strong and loose coupling.

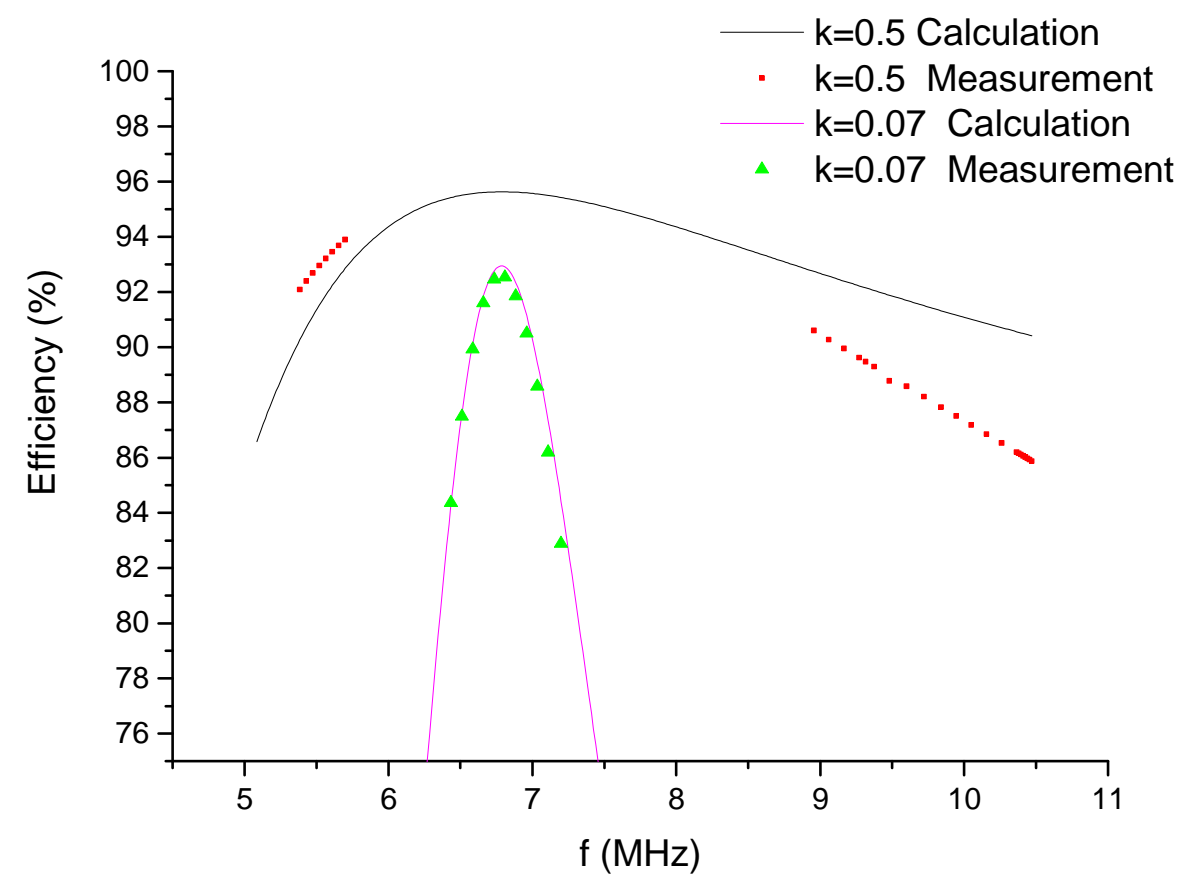

Figure 5.7: Frequency splitting effect (Efficiency drop at $\omega_{1}$ and $\omega_{2}$ : calculation and testing results.).

The efficiency of a wireless power transfer system, which is not operating in at the resonant frequency, is given by

$$
\eta=\eta_{1} \eta_{2}=\frac{R_{L}}{R_{1} \frac{\left(R_{2}+R_{L}\right)^{2}+\left(\omega_{0} L_{2} \Delta\right)^{2}}{\omega^{2} M^{2}}+R_{2}+R_{L}}
$$


where

$$
\Delta=\frac{\omega}{\omega_{0}}-\frac{\omega_{0}}{\omega}
$$

From Equations (5.38) and (5.39), it can be seen that the efficiency decreases as the operating frequency $(\omega)$ deviates from the resonant frequency $\left(\omega_{0}\right)$. To replace the $\omega_{0}$ of Equation (5.38) with Equation (5.11), the efficiency at $\omega_{1}$ is given by

$$
\eta_{\omega_{1}}=\frac{R_{L}\left(\omega_{0}^{2} L_{2}^{2} k^{2}\right)}{(1+k) R_{1}\left[\left(R_{2}+R_{L}\right)^{2}+\left(\omega_{0} L_{2}\right)^{2}\left(\frac{1}{\sqrt{1+k}}-\sqrt{1+k}\right)^{2}\right]\left(\omega_{0}^{2} L_{2}^{2} k^{2}\right)+\left(R_{2}+R_{L}\right)\left(\omega_{0}^{2} L_{2}^{2} k^{2}\right)}
$$

It can be seen from the derived equation that the efficiency at $\omega_{1}$ is related to the coupling coefficient and the coil inductance. Figure 5.8 shows the change in efficiency with varying coupling coefficients for different coil inductances.

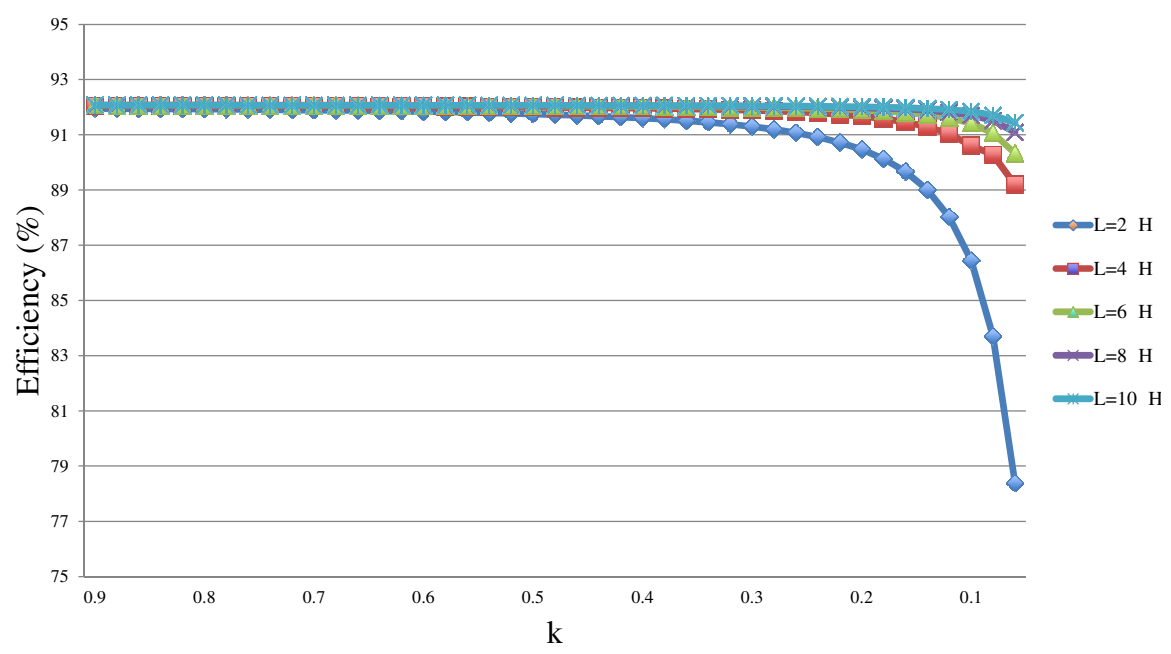

Figure 5.8: Efficiency at $\omega_{1}$ for different coupling value.

Figure 5.8. shows that when the coupling is strong, the efficiency at $\omega_{1}$ is high regardless of the value of the coil inductance. However, when the coupling is loose, the efficiency of the system with low inductance value is not high enough. This therefore suggests that the value of the inductance of $4 \mu \mathrm{H}$ is used in this thesis. 
For a $6.78 \mathrm{MHz}$ wireless power transfer system, a planar coil is always utilized because of its compact structure. The equation for the inductance of a coil is given in [140], [141], [142], [143] and [144] as

$$
L=\frac{1}{2} N^{2} \mu d_{a v g} C_{1}\left(\ln \frac{C_{2}}{F}+C_{3} F+C_{4} F^{2}\right)
$$

where $\mathrm{N}$ is the number of turns, $\mu$ is the permeability of the material, $d_{a v g}$ is the average diameter and $C_{1}-C_{4}$ are the parameters related to the shape of the coil. According to [140], $C_{1}-C_{4}$ of a coil that is of a square shape is larger than those of a circular coil. In view of that, a square shaped coil is chosen in this design.

Another important parameter of the coil is its resistance, especially when the coil works at high frequency. The AC resistance of the coil is mainly determined by two factors: the skin effect and the proximity effect. To mitigate skin effect losses, the thickness of the coil should be designed to be larger than the skin depth. To alleviate the proximity effect, the space between each coil turn should be as least at least one diameter apart.

\subsubsection{Design of a Zero Voltage Switching (ZVS) CLASS-D Amplifier}

Another challenging issue with the $6.78 \mathrm{MHz}$ wireless power transfer system is the significant power loss of the power amplifier at the transmitter end, due to the high operating frequency of the system. To reduce the switching losses, a full-bridge zero voltage switching (ZVS) class D amplifier is adopted in this design ( [145], [146], [147], [148], [149], [150], [151] and [152] ).

Figure 5.9 shows a basic topology of a typical full-bridge ZVS class D amplifier and its corresponding operating waveforms [147]. The GaN transistors $\left(A_{1}\right.$, $A_{2}, B_{1}$ and $B_{2}$ ) form the power stage of the amplifier; the inductance $L_{z v s}$ is used to store the energy for ZVS operation; $L_{1}$ and $C_{1}$ is the resonator including the coil and the compensation capacitor; $Z_{r}$, as described before, is the equivalent reflected impedance of the entire receiver including the receiving coil, compensation capacitor, rectifier, DC-DC converter and the load. 


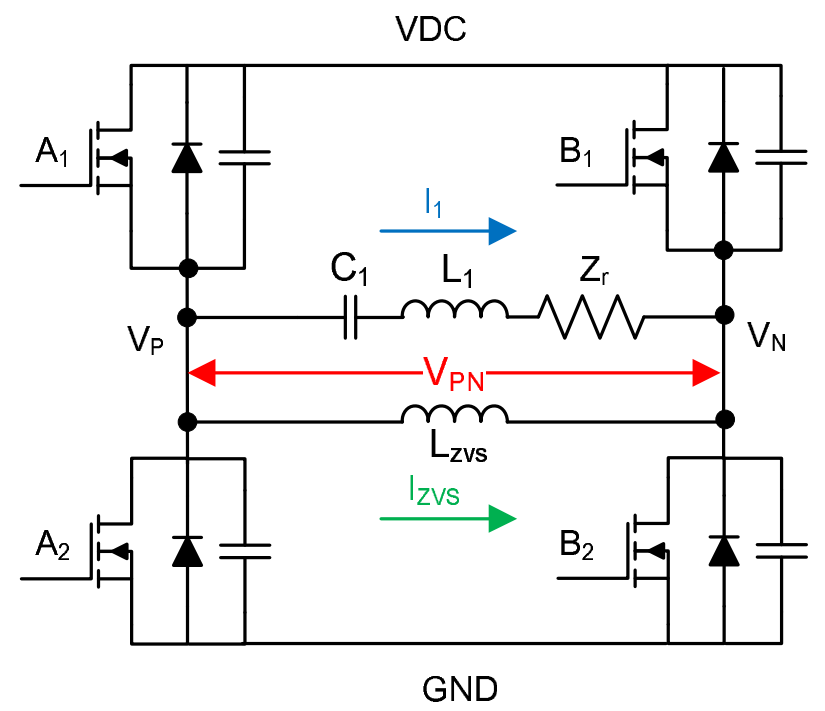

Figure 5.9: ZVS Class-D Amplifier.

The operating mechanism of the amplifier is depicted in Figure 5.10. The overall operation of the ZVS class-D amplifier period can be segmented into four phases, as presented below.

In Phase I, the two diagonally positioned GaN transistors, $A_{1}$ and $B_{2}$, are conducting. The current of the coil $L_{1}$ flows through the two transistors to transfer power to the load. The $V_{P N}$ is positive hence causing the current in $L_{z v s}$ to increase. The current stored in the $L_{z v s}$ will ultimately be used to realize zero voltage switching.

During Phase II, transistor $B_{2}$ shuts down. At this point, the current to load should be close to zero if it is well compensated. The current in $L_{z v s}$ begins to charge the capacitance of $B_{2}$ while discharging $B_{1}$. This phase should end once the voltage across $B_{1}$ is discharged to zero. Otherwise, the current will flow through the diode of $B_{1}$ causing unnecessary power loss.

In Phase III, transistor $B_{1}$ is turned on and $A_{1}$ is turned off after that. Since the voltage across $B_{1}$ is during Phase II was at zero level, the switching operation is considered as ZVS. $I_{1}$ is still close to zero and $I_{z v s}$ begins to discharge the capacitance of $A_{2}$ until the voltage across $A_{2}$ is zero.

In Phase IV, $A_{2}$ turns on. Since the voltage across it is zero in Phase III, it is also considered that ZVS is in action. The current of the coil $L_{1}$ goes through $A_{2}$ 


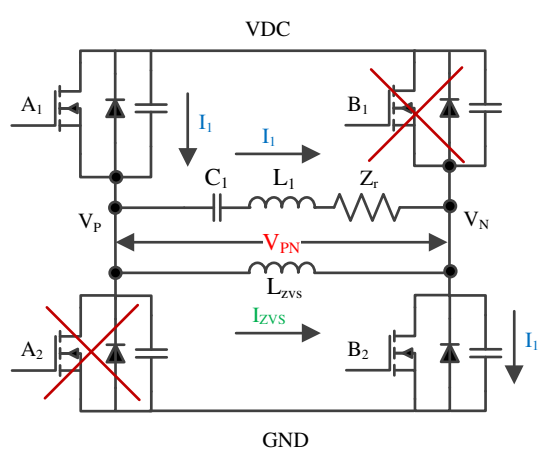

Phase I

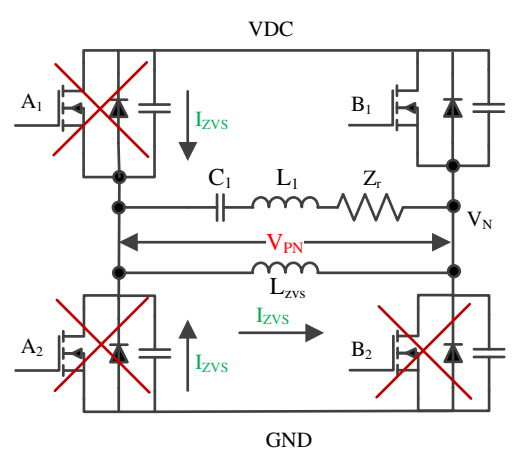

Phase III

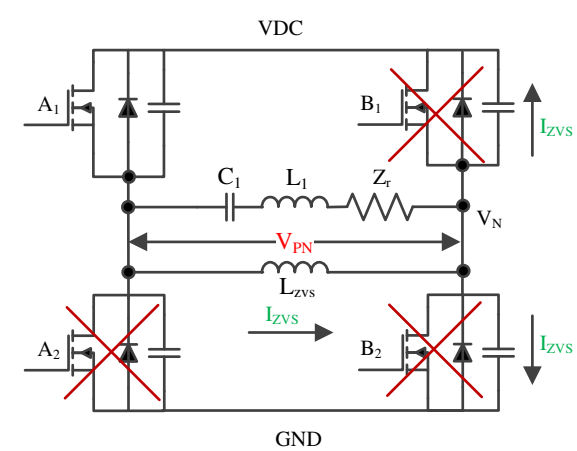

Phase II

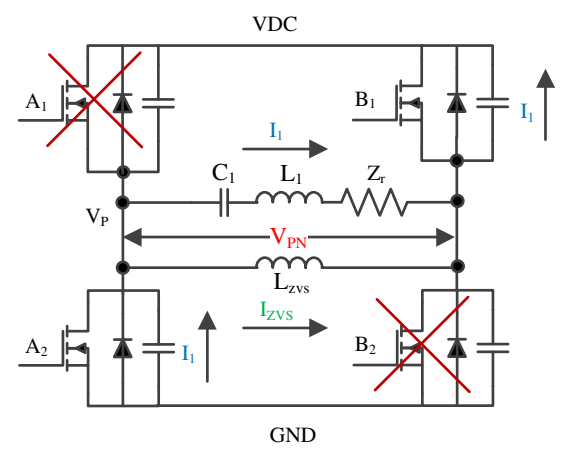

Phase IV

Figure 5.10: Operation of the ZVS.

and $\mathrm{B} 1$ to transfer power to the load. The VPN is negative triggering the current in $L_{z v s}$ to decrease. The current stored in $L_{z v s}$ will be used to realize the ZVS action of $A_{1}$ and $B_{2}$ in the next switching action.

The waveform of the ZVS class-D amplifier is shown in Figure 5.11. It can be seen that in Phase I, the current of the $L_{z v s}$ is built by VDD, given by

$$
I_{Z V S}=\frac{1}{2 L_{Z V S}} \int_{t_{Z V S}}^{\frac{1}{2} T-t_{Z V S}} V D D \cdot d t=\frac{V D D\left(T-4 t_{Z V S}\right)}{4 L_{Z V S}}
$$

According to the analysis above, the $I_{z v s}$ should discharge the $C_{O S S Q}$, which is the equivalent output capacitance of the GaN devices, in the time duration $t_{z v s}$. Since $t_{z v s}$ is short so that $I_{z v s}$ can be considered as constant during $t_{z v s}$. It can be derived that:

$$
L_{z v s} t_{z v s}=C_{O S S Q} V D D
$$




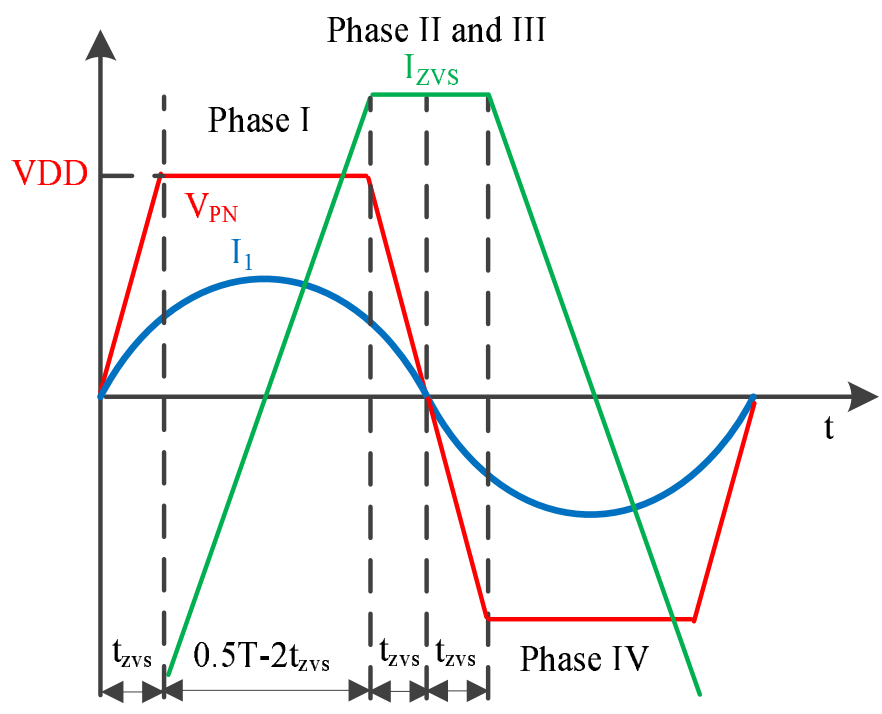

Figure 5.11: Voltage-Current waveform of the ZVS class-D amplifier.

According to Equation 5.43, $L_{z v s}$ is thus expressed as

$$
L_{z v s}=\frac{t_{z v s}\left(T-4 t_{z v s}\right)}{4 C_{O S S Q}}
$$

\subsection{Experimental Results}

The experimental test bench (Figure 5.12) was built based on the parameters tabulated in Table 5.2. Experimentations were carried out to verify and evaluate the proposed voltage regulation and efficiency optimization methodologies of the magnetic resonant wireless power transfer system. A full-bridge class-D ZVS power amplifier is incorporated at the transmitting end to generate a $6.78 \mathrm{MHz}$ AC voltage to drive the transmitting coil. The DC input voltage for the amplifier is fixed at $12 \mathrm{~V}$, which is the required minimum voltage for long-distance WPT. As described above, both the operating frequency and phase shift are tuned by a MCU (MICROCHIP DSPIC33FJ16GS402-I) with a high speed PWM function.

Testing Case I: Figure 5.13 shows the output waveform of the class-D amplifier in the steady state when the system is working at a $5 \mathrm{~cm}$ distance (loose coupling) and $10 \mathrm{ohm}$ load. It can be seen that the output pulse width is close to $100 \%$ at resonant frequency and that the output voltage across the load is around 


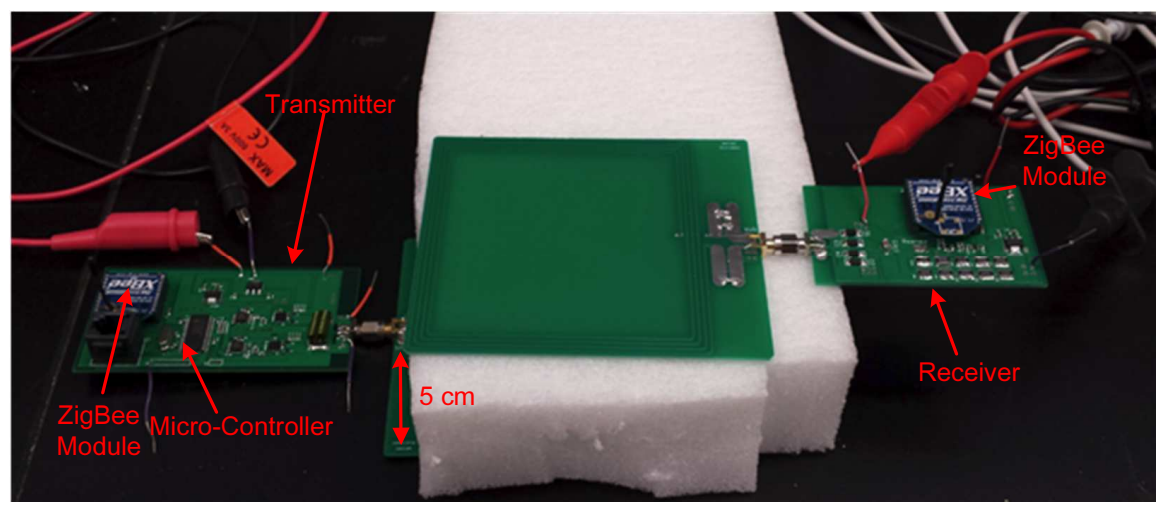

Figure 5.12: Test bench for the proposed 6.78-MHz WPT system.

Table 5.2: Testing Components of the proposed 6.78-MHz WPT system

\begin{tabular}{|c|c|}
\hline Circuit Components & Value \\
\hline Power Transistors & EPC2014C \\
\hline Transmitting coil & $4.1 \mu \mathrm{H} / 0.43$ Ohms @ 6.78 MHz \\
\hline Receiving coil & $4.1 \mu \mathrm{H} / 0.43$ Ohms @ 6.78 MHz \\
\hline Compensation Capacitor & $100 \mathrm{pF} / 500 \mathrm{~V} / / 30 \mathrm{pF} / 500 \mathrm{~V} / / 6.9 \mathrm{pF} / 500 \mathrm{~V}$ \\
\hline Smoothing capacitor & $22 \mu \mathrm{F} / 50 \mathrm{~V}$ \\
\hline Micro-controller & MICROCHIPDSPIC33FJ16GS402-I \\
\hline Communication Module. & XBee by Digi \\
\hline
\end{tabular}

$10 \mathrm{~V}$. It can be seen that the frequency is close to $6.78 \mathrm{MHz}$ (period $=148 \mathrm{~ns}$ ), which implies that the system works under phase shift tuning mode at the resonant frequency.

Testing Case II: If the distance and the DC input voltage are kept the constant at $5 \mathrm{~cm}$ and $12 \mathrm{~V}$, respectively, and the load is $50 \mathrm{ohms}$, the controller will change the duty cycle of the output voltage of the amplifier according to the feedback signals as what has been described as the control flow in Figure 5.6. At the beginning, the duty cycle is about $70 \mathrm{~ns}$ as can be seen from Figure 5.13. The controller then progressively controls the duty cycle to approximately $20 \mathrm{~ns}$ as shown in Figure 5.14. Meanwhile, it is corroborated that the output DC voltage is successfully regulated to maintain at around $10 \mathrm{~V}$. The voltage regulation time from Testing Case I to Testing Case II is around $300 \mathrm{~ms}$.

Testing Case III:If the load is kept at $10 \mathrm{Ohms}$ but the power transfer distance shortened to $1 \mathrm{~cm}$. The system will then work in strong coupling mode and 


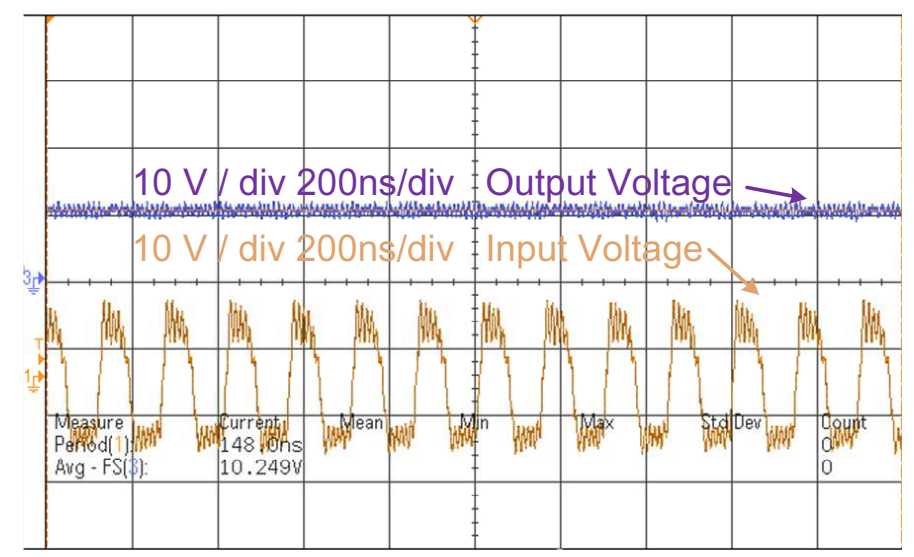

Figure 5.13: Oscilloscope waveform of the output voltage cross the load $\left(R_{L}=10\right.$ $\Omega$ ) and the power amplifier output voltage at $5 \mathrm{~cm}$.

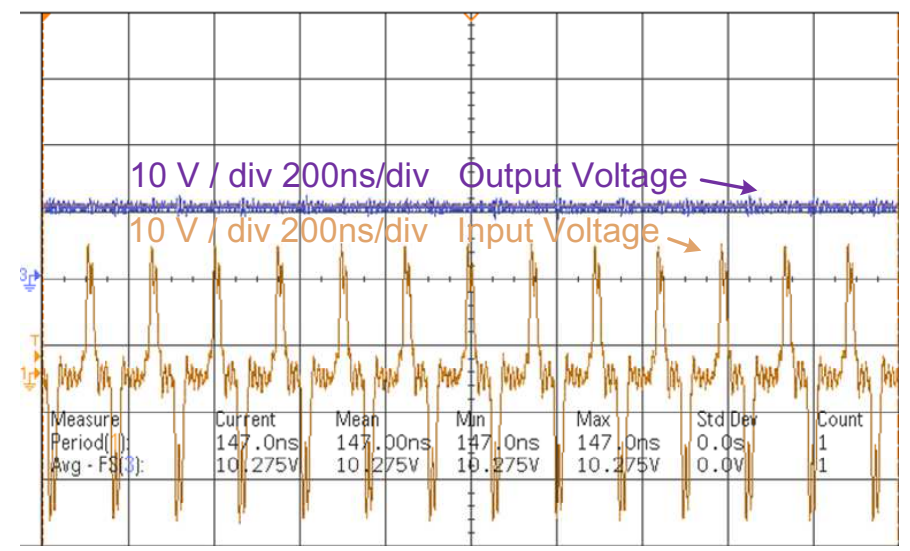

Figure 5.14: Oscilloscope waveform of the output voltage cross the load $\left(R_{L}=50\right.$ $\Omega$ ) and the power amplifier output voltage at $5 \mathrm{~cm}$.

frequency splitting phenomenon will adversely impact the power transfer capability. In line with the control flow chart depicted in Figure 5.6, the controller tunes the operating frequency lower once it detects that it is impossible for the system working under resonant frequency. As shown in Figure 5.15, the operating frequency is tuned to $5.65 \mathrm{MHz}$ (period= $177.5 \mathrm{~ns}$ ). Meanwhile, the output DC voltage is maintained at around $10 \mathrm{~V}$. The voltage regulation time from Testing Case I to Testing Case III is around $400 \mathrm{~ms}$.

Testing Case IV: If the load is increased from $10 \mathrm{Ohms}$ to $50 \mathrm{Ohms}$ under similar strong coupling condition, once the controller detects an overflow output DC voltage, it tunes the operating frequency closer to the resonant frequency in an effort to regulate the output voltage at a desired level $10 \mathrm{~V}$. The waveforms are 


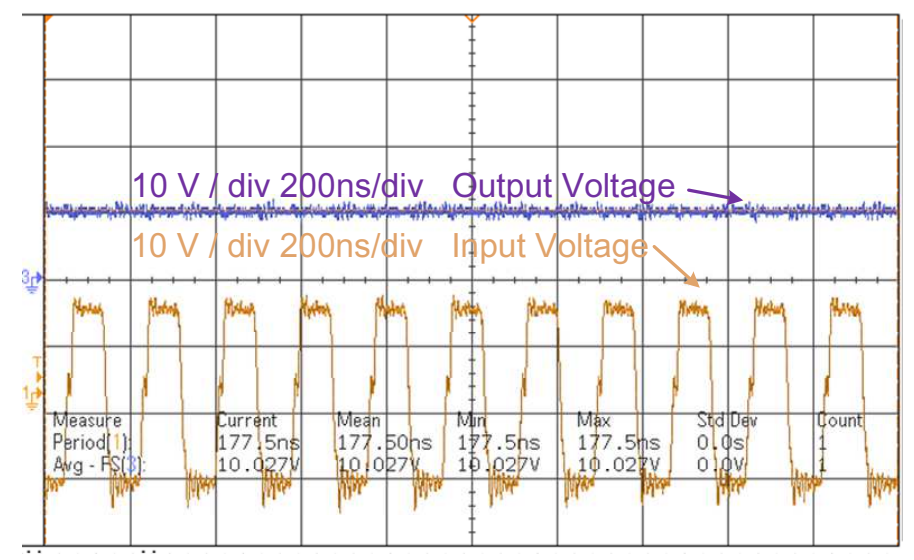

Figure 5.15: Oscilloscope waveform of the output voltage cross the load $\left(R_{L}=10\right.$ $\Omega$ ) and the power amplifier output voltage at $1 \mathrm{~cm}$.

shown in Figure 5.16. In the steady state, the output DC voltage was maintained at around $10 \mathrm{~V}$ and the frequency was tuned to $5.92 \mathrm{MHz}$ (period= $168.8 \mathrm{~ns}$ ). The voltage regulation time from Testing Case III to Testing Case IV is around $350 \mathrm{~ms}$.

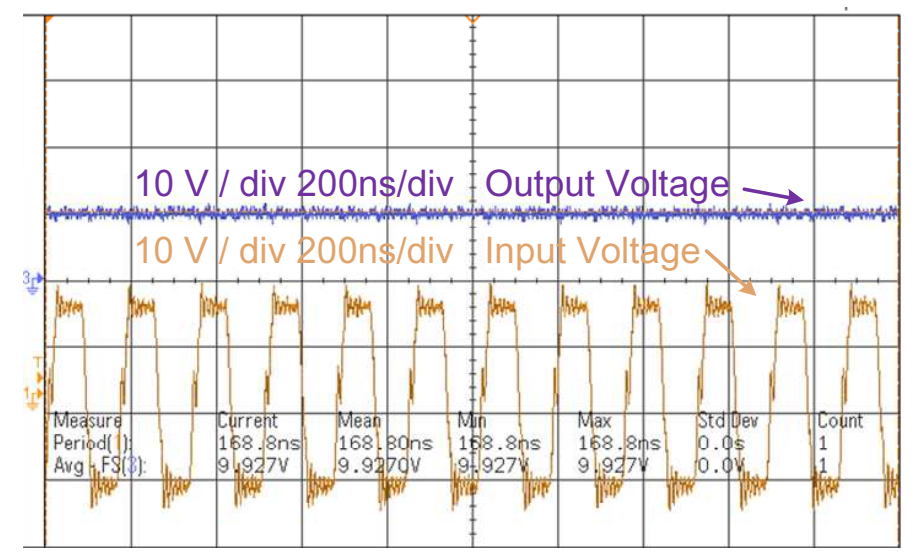

Figure 5.16: Oscilloscope waveform of the output voltage cross the load $\left(R_{L}=50\right.$ $\Omega$ ) and the power amplifier output voltage at $1 \mathrm{~cm}$.

The overall system efficiency is tested based on varying coupling and load conditions. The results of both the overall efficiency and break-down efficiency are shown in Table 5.3 and the comparison with other works is shown in Table 5.4. It can be seen the proposed WPT system has the voltage regulation function and can be applied in a wide dynamic transferring distance. It also can be seen that in case II, the efficiency of the Class-D amplifier drops to $55.1 \%$ due to the free-wheeling conduction of the phase-shift modulation [77], while in case II and 
IV the efficiency of class D drops to around $50 \%$ since the switching loss do not decrease as the output power decrease.

Table 5.3: Testing results of the proposed WPT system

\begin{tabular}{|c|c|c|c|c|}
\hline Testing Cases & $\begin{array}{c}\text { DC-AC } \eta \\
(\text { Class D } \eta)\end{array}$ & $\begin{array}{c}\text { AC-AC } \eta \\
(\text { Coil-Coil } \eta \text { ) }\end{array}$ & $\begin{array}{c}\text { AC-DC } \eta \\
(\text { Rectifier } \eta \text { ) }\end{array}$ & Overall $\eta$. \\
\hline I:10 Ohm @ 5cm & $85.5 \%$ & $91.2 \%$ & $92.2 \%$ & $71.8 \%$ \\
\hline II:50 Ohm @ 5cm & $55.1 \%$ & $84.6 \%$ & $92.0 \%$ & $42.7 \%$ \\
\hline III:10 Ohm @ 1cm & $86.3 \%$ & $93.1 \%$ & $91.1 \%$ & $73.1 \%$ \\
\hline IV: 50 Ohm @ 1cm & $50.2 \%$ & $96.6 \%$ & $92.1 \%$ & $44.6 \%$ \\
\hline
\end{tabular}

Table 5.4: Performance comparison with the proposed WPT system

\begin{tabular}{|c|c|c|c|c|c|c|}
\hline Ref. & TBCAS & AWPL & TIA & JSSC & TPEL & This \\
& $\begin{array}{c}2008 \\
{[153]}\end{array}$ & $\begin{array}{c}2014 \\
{[110]}\end{array}$ & $\begin{array}{c}2014 \\
{[51]}\end{array}$ & $\begin{array}{c}2015 \\
{[44]}\end{array}$ & $\begin{array}{c}2016 \\
{[138]}\end{array}$ & Work \\
\hline $\begin{array}{c}\text { Varying } \\
\text { Dist.? }\end{array}$ & Yes & Yes & Yes & Yes & No & Yes \\
\hline Dist. & $\leq 1 \mathrm{~cm}$ & $1-1.4 \mathrm{~cm}$ & $15-35 \mathrm{~cm}$ & $0.3-1 \mathrm{~cm}$ & N.A. & $1-5 \mathrm{~cm}$ \\
\hline$P_{M A X}$ & $12 \mathrm{~W}$ & $9 \mathrm{~W}$ & N.A. & $102 \mathrm{~mW}$ & $20 \mathrm{~W}$ & $10 \mathrm{~W}$ \\
\hline Freq. & $60-70$ & $270-$ & $3.8-$ & 13.56 & 6.78 & 6.78 \\
& $\mathrm{kHz}$ & $330 \mathrm{kHz}$ & $6 \mathrm{MHz}$ & $\mathrm{MHz}$ & $\mathrm{MHz}$ & $\mathrm{MHz}$ \\
\hline Control? & Yes & No & No & Yes & No & Yes \\
\hline$V_{\text {out }}$ & $3 \mathrm{~V}$ & N.A. & N.A. & $3.6 \mathrm{~V}$ & $22 \mathrm{~V}$ & $10 \mathrm{~V}$ \\
\hline Effic. & $80 \%$ & $90 \%$ & $65 \%$ & $50 \%$ & $84 \%$ & $71 \%$ \\
& $@ 0.5 \mathrm{~cm}$ & $@ 1.5 \mathrm{~cm}$ & $@ 35 \mathrm{~cm}$ & $@ 0.3 \mathrm{~cm}$ & $@ \mathrm{k} 0.13$ & $@ 5 \mathrm{~cm}$ \\
& DC-DC & Coil-Coil & Coil-Coil & DC-DC & DC-DC & DC-DC \\
\hline
\end{tabular}

\subsection{Conclusions}

In this chapter, we present a detailed empirical analysis on power transfer efficiency and output power of a magnetic resonant wireless power transfer system for both strong and weak coupling modes. It has been derived that the frequencies of output power peak points when frequency splitting happen are only related to the coupling coefficient. In addition, an effective voltage regulation control flow has been developed and experimentally demonstrated to be capable of regulating the output voltage at a constant level over a wide range of transmission distance 
$(1 \mathrm{~cm}-5 \mathrm{~cm})$. Meanwhile, the system efficiency also optimized. The proposed wireless power transfer system is able to transfer $10 \mathrm{~W}$ over a $5 \mathrm{~cm}$ with an overall efficiency of $71.6 \%$, and over a $1 \mathrm{~cm}$ with an overall efficiency of $73.1 \%$. Compared to the state-of-art technology, the proposed WPT system has a high efficiency within a wide range of coupling conditions. 



\section{Chapter 6}

\section{Conclusions and Future Work}

In this chapter, the main contributions of this research project are summarized and the conclusions of the thesis are presented. Recommendations for the future work of this research project is also proposed.

\subsection{Conclusions}

In recent years, wireless power transfer (WPT) technology has become more and more popular as it has been touted as a viable solution for non-contact charging of electronic appliances. However, the performance of the technology is still far from satisfying the requirements of the mass consumers market.

The main target of this thesis is to improve the performance of wireless power transfer (WPT) system for consumer electronic devices, particularly in terms of high performance circuits design and the smart control method design. The background of the three main categories of wireless power transfer technology: Electromagnetic (EM) wave WPT, induction WPT and magnetic WPT, and the main building blocks of a WPT system, for instance DC/AC converter, compensation network, rectifier, DC/DC converter and their corresponding control algorithm have been comprehensively reviewed. It has been found it is challenging to design high performance circuits and smart control solutions, and there is still a serious lack in low cost high performance AC-DC converter, reverse leakage control and smart control which can overcome the frequency splitting mentioned in 


\section{Chapter 5.}

In this dissertation, a single stage $\mathrm{AC} / \mathrm{DC}$ voltage regulator for the induction WPT has been proposed, which is to replace the traditional rectifier plus DC-DC converter or voltage regulation with wireless communication approach. The cross-coupled full active rectifier has been adopted and a fast response comparator has been used to eliminate the reverse leakage current. The power loss, voltage gain and transfer function is analyzed. A voltage PWM control is used to provide the regulator with a constant and stable $5 \mathrm{~V}$ output. The operating frequency of the WPT is $125 \mathrm{kHz}$ and the switching frequency of the regulator is $7 \mathrm{kHz}$. This novel regulator design with the proposed control mechanism was fabricated based on AMS $0.35 \mu \mathrm{m}$ high voltage CMOS technology. For measurement and testing of the regulator, the load resistance was adjusted between 1.6 Ohms and $25 \mathrm{Ohms}$ and the output voltage ripple was maintained at about 40 $\mathrm{mV}$. The achievable peak efficiency of the regulator is at $92 \%$ and this value can be further improved if a $\mathrm{BCD}$ process or $\mathrm{GaN}$ devices is used. When compared to the existing works, this regulator is a true single stage design without extra DC-DC converter and other peripheral communication circuits.

Subsequently, a full active rectifier for the magnetic wireless power transfer application has also been designed and physically implemented. The proposed full digital adaptive delay control is able to work for a WPT system at $6.78 \mathrm{MHz}$ operating frequency. A SAR algorithm is used to align the gate driving signal to the $\mathrm{AC}$ input voltage. As a result, the reverse leakage current is eliminated, which in turn successfully optimized the efficiency of the rectifier. To further verify the proposed full active rectifier, this design was fabricated in AMS $0.18 \mu \mathrm{m}$ CMOS technology. Measurements were carried out based on a wide output power range from $15 \mathrm{~W}$ to $1 \mathrm{~W}$. The fabricated rectifier has a peak efficiency of $90 \%$ at the output of $5 \mathrm{~V}$ and $2 \mathrm{~A}$. It has been proven that this method effectively removed the reverse leakage of the rectifier.

Finally, a magnetic WPT system coupled with adaptive voltage regulation 
and efficiency optimization capability for $15 \mathrm{~W}$ applications has been developed. For a high frequency WPT system in a strong coupling mode, the power transfer capability of the system at the resonant frequency is pulled down due to the frequency splitting phenomena. Conversely, while in the loosely coupling mode, the system has to work at resonant frequency to maintain the efficiency at a high level. A new voltage regulation technique aimed at circumventing the frequency splitting issue and enhancing efficiency optimization has been developed to solve this problem. Besides, a zero voltage switching class D amplifier is designed to decrease the power loss of the DC/AC stage. This design has been verified using GaN devices, micro-controller, and ZigBee communication models and it has been corroborated that the system is able to provide a constant $10 \mathrm{~V}$ from $15 \mathrm{~W}$ to $1 \mathrm{~W}$ and capable of achieving peak efficiency of $73 \%$. This control method is able to provide a stable output voltage and keep the performance (power transfer efficiency and capability) of the system at a high level. To the best of our knowledge, this is the first time a WPT system with such functions has ever been developed and physically implemented.

\subsection{Future Work}

For the single stage regulator, the efficiency for light load condition still has room for improvement. One possible solution is to use envelope detection method to detect the duty-cycle of charging-mode and zero-mode so as to adjust the output voltage of the AC/DC converter. In light load condition, the power loss in zero-mode, which is mentioned in Chapter 3, deteriorates the efficiency. In that case, the AC/DC converter is supposed to be able to reduce the output voltage to reduce the zero-mode duty-cycle.

For the $6.78 \mathrm{MHz}$ wireless power transfer system, the ZVS class D amplifier could be further improved. Since in the phase shift tuning operation, there is a freewheel duration for light load condition, the power loss in this duration will pull down the efficiency. This could be solved by changing the input voltage in- 
stead of changing the phase shift. Meanwhile, although the power consumption of ZigBee communication is lower than Bluetooth communication, it is slower in the meantime. In that case, it is possible to consider using Bluetooth communication for future designs. 



\section{APPENDIX A}

\section{Phasor Transformation}

Phasor transformation is used to transfer a rotationary AC circuit into a equivalent DC circuit model. The basic principle of the Phasor transformation is to find a DC stationary sub-circuit model for each AC rotationary circuit components, which makes it much easier to find the value of envelopes of AC rotationary variables in an AC rotationary circuit. Some traditional transformation like D-Q transformation $[\mathrm{DQ}]$ can only solve the switching and transformer transformation, but not able to deal with the inductor, capacitor and so on. So in this thesis, the phasor transformation is adopted. To build a valid model for WPT system, the equivalent component model has to be able to reflect not only the magnitude and the phase of a sinusoidal signal in the steady state state but also the response of the transient state.The phasor which can represent any sinusoidal signal is:

$$
X(t) \equiv \mathfrak{R}\left[\sqrt{2} x(t) e^{(j \omega t)}\right]
$$

where the $x(t)$ indicates the complex time-varying variable. It is worth to note that the $x(t)$ and $\omega$ are not necessary to be sinusoidal or constant but also can be non-sinusoidal function only when the $x(t)$ is sinusoidal with the frequency of $\omega$. In the steady state, $x(t)$ becomes just the conventional phasor. The three basic circuit elements of the WPT system: inductor, capacitor and resistor, are phasor-transformed respectively, as follows. 
Inductor Phasor Transformation

The procedure of the phasor transformation for the inductor is shown in Figure A.1. The voltage-current circuit equation is

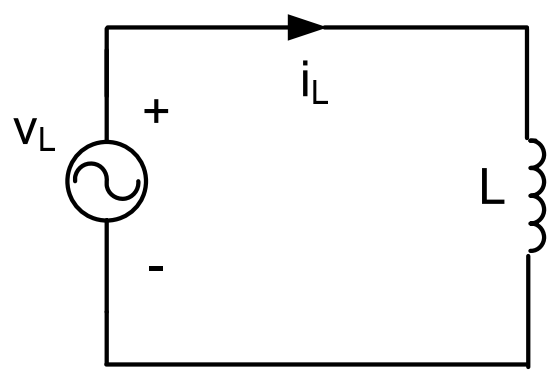

(a)

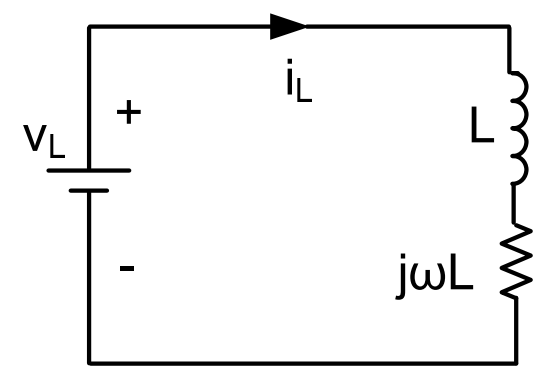

(b)

Figure A.1: Inductor phasor transformation. (a) AC-Rotationary circuit. (b) DCStationary circuit.

To transform the current and voltage of the inductor to phasor, it would have:

$$
\left\{\begin{array}{l}
i_{L} \equiv \mathfrak{R}\left[\sqrt{2} i_{L} e^{(j \omega t)}\right] \\
v_{L} \equiv \Re\left[\sqrt{2} v_{L} e^{(j \omega t)}\right]
\end{array}\right.
$$

Applying Equation (A.2) to Equation (A.1) results in

$$
\begin{aligned}
L \frac{d}{d t}\left[\Re\left[\sqrt{2} i_{L} e^{(j \omega t)}\right]\right] & =\Re\left[\sqrt{2} v_{L} e^{(j \omega t)}\right] \\
\text { or } \quad L \Re\left[\frac{d i_{L}}{d t} e^{(j \omega t)}+j \omega i_{L} e^{(j \omega t)}\right] & =\Re\left[\left(\frac{d i_{L}}{d t}+j \omega i_{L}\right) e^{(j \omega t)}\right]=\Re\left[v_{L} e^{(j \omega t)}\right] .
\end{aligned}
$$

Equation (A.3) is just equivalent to

$$
L \frac{d i_{L}}{d t}+j \omega L i_{L}=v_{L}
$$

The equivalence of Equation (A.4) with Equation (A.3) can be proved as follows.

Theorem

For any $\mathrm{x}, \mathrm{y}$ and $\omega \neq 0, \Re\left[x e^{(j \omega t)}\right]=\mathfrak{R}\left[y e^{(j \omega t)}\right]$, if and only if $\mathrm{x}=\mathrm{y}$. 
Proof

$$
\begin{aligned}
\Re\left[x e^{(j \omega t)}\right] & =\Re\left[|x| e^{(j \arg (x)} e^{(j \omega t)}\right] \\
& =|x| \Re\left[e^{(j \arg (x)+j \omega t)}\right] \\
& =|y| \Re\left[e^{(j \arg (y)+j \omega t)}\right] \\
\leftrightarrow|x| & =|y| \quad \operatorname{andarg}(x)=\arg (y)+2 \pi n, n=0,1,2 \ldots \\
\leftrightarrow x & =y
\end{aligned}
$$

It is gain from Equation (A.4) the form of circuit in Figure. A.1(a) could be transferred to Figure. A.1(b), for establish DC analysis for the WPT system. The imaginary resistor, $j \omega L$, the meaning of which is just conventional inductor impedance in the steady state. However it should be noticed that this circuit model in Figure. A.1(b) is still valid in transient analysis for the DC stationary circuit model. The imaginary resistor does not vanish even though it is in the transient state.

Capacitor Phase Transformation By a similar procedure with the inductor case, the rotationary circuit equation of Figure A.2(a),

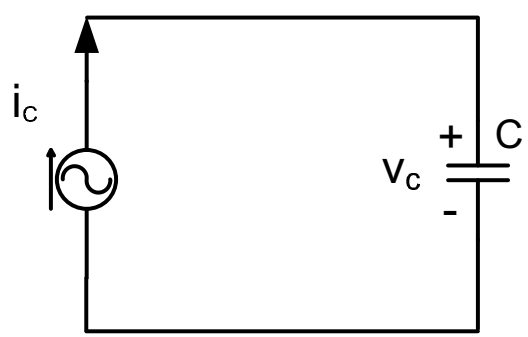

(a)

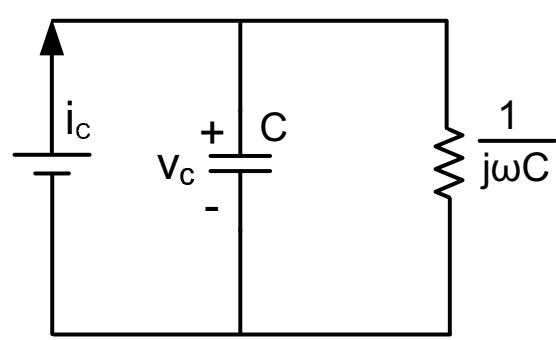

(b)

Figure A.2: Capacitor phasor transformation. (a) AC-Rotationary circuit. (b) DC-Stationary circuit.

is changed by the phasor transformation to 


$$
C \frac{d v_{C}}{d t}+j \omega C v_{C}=i_{C}
$$

The circuit model of the DC stationary model of Equation A. 2 is shown in Figure A.2(b).

\section{Resistor Phasor Transformation}

The phasor transformation of a resistor, as shown in Figure A.3 is straightforward since

$$
\begin{aligned}
v_{R}=\Re\left[\sqrt{2} v_{R} e^{(j \omega t)}\right] & =i_{R} R=\Re\left[\sqrt{2} i_{R} e^{(j \omega t R)}\right], \\
\text { or } \quad v_{R} & =i_{R} R .
\end{aligned}
$$

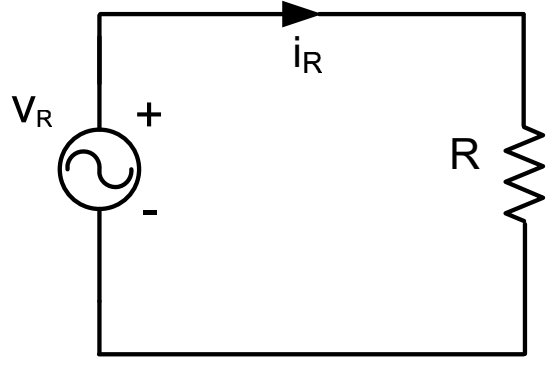

(a)

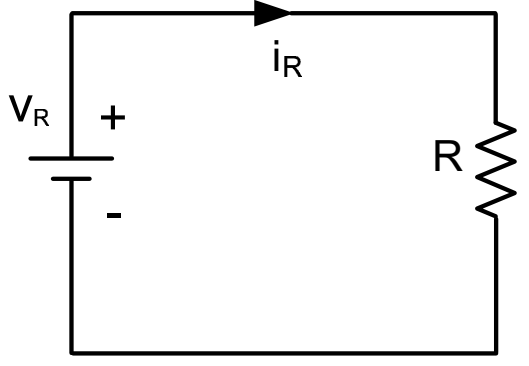

(b)

Figure A.3: Resistor phasor transformation. (a) AC-Rotationary circuit. (b) DCStationary circuit. 


\section{APPENDIX B}

\section{Author's Publications}

\section{B.1 Journal Papers}

- Xiaoyin Bai, Zhi Hui Kong and Liter Siek, "A Single-Stage AC-DC Voltage Regulator for 15W Wireless Charging," submitted to IET Electronics letter.

- Xiaoyin Bai, Zhi Hui Kong and Liter Siek, "A High-Efficiency $6.78 \mathrm{MHz}$ Full Active Rectifier with Adaptive Delay Control for Wireless Power Transmission," Accepted by IEEE Transactions on Very Large Scale Integration (VLSI) Systems.

- Xiaoyin Bai, Zhi Hui Kong and Liter Siek, "Analysis and Hybrid Control Method for Wireless Power Transfer System with Varying Transmission Distance," submitted to IEEE Antennas and Wireless Propagation Letters.

\section{B.2 Patent Proposals}

- Xiaoyin Bai, Zhi Hui Kong, Liter Siek and Tan Yen Kheng, "A 92\% Efficiency 15 W Single-Stage Wireless Charging Receiver With 5 V DC Voltage Regulation",SG Provisional Patent Application No. 10201404736R had been re-filed in 2016 Jan . 
- Xiaoyin Bai, Zhi Hui Kong, Liter Siek and Tan Yen Kheng, "A SingleStage AC-DC Voltage Regulator for Wireless Charging",SG Provisional Patent Application No. 10201404736R. Filed in 2014 September.

\section{B.3 Conference Papers}

- Xiaoyin Bai, Zhi-Hui Kong and Liter Siek, "A novel control method for magnetic wireless charging system", IEEE International Conference on Electron Devices and Solid-State Circuits (EDSSC). June. 2015

- Xiaoyin Bai, Yen-Kheng Tan, Zhi-Hui Kong and Liter Siek, "Novel Active Tuning Approach for Resonant-mode Wireless Charging System", IEEE International Conference on Industrial Technology (ICIT). Feb.-March. 2014

- Xiaoyin Bai, Yen-Kheng Tan, Tran Duong, Zhi-Hui Kong and Siek Liter, "A Wireless Charging Resonant Frequency Checking Technique for LVDC", Asia Smart Grid 2013. Oct. 2013 


\section{BIBLIOGRAPHY}

[1] N. Tesla, “Apparatus for transmitting electrical energy." Dec. 11914 , uS Patent 1,119,732. [Online]. Available: http://www.google.com/patents/ US1119732

[2] W. C. Brown and E. Eves, "Beamed microwave power transmission and its application to space," Microwave Theory and Techniques, IEEE Transactions on, vol. 40, no. 6, pp. 1239-1250, Jun 1992.

[3] M. J. McSpadden JO, "Space solar power programs and microwave wireless power transmission technology," Microwave Magazine, IEEE, vol. 3, no. 4, pp. 46-57, Dec 2002.

[4] J. Benford, "Space applications of high power microwaves," in Pulsed Power Conference, 2007 16th IEEE International, vol. 1, June 2007, pp. 258-265.

[5] G. Chattopadhyay, H. Manohara, M. Mojarradi, T. Vo, H. Mojarradi, S. Bae, and N. Marzwell, "Millimeter-wave wireless power transfer technology for space applications," in Microwave Conference, 2008. APMC 2008. Asia-Pacific, Dec 2008, pp. 1-4.

[6] A. Sample, D. Yeager, P. Powledge, A. Mamishev, and J. Smith, "Design of an rfid-based battery-free programmable sensing platform," Instrumentation and Measurement, IEEE Transactions on, vol. 57, no. 11, pp. 26082615, Nov 2008.

[7] W. C. Brown, "The history of power transmission by radio waves," Microwave Theory and Techniques, IEEE Transactions on, vol. 32, no. 9, pp. 1230-1242, Sep 1984.

[8] M. Catrysse, B. Hermans, and R. Puers, "An inductive power system with integrated bi-directional data-transmission," Sensors and Actuators A: Physical, vol. 115, no. 2, pp. 221 - 229, 2004, the 17th European Conference on Solid-State Transducers. [Online]. Available: http://www.sciencedirect.com/science/article/pii/S0924424704001335

[9] J. Sallan, J. Villa, A. Llombart, and J. Sanz, "Optimal design of icpt systems applied to electric vehicle battery charge," Industrial Electronics, IEEE Transactions on, vol. 56, no. 6, pp. 2140-2149, June 2009.

[10] J. C. Schuder, "Powering an artificial heart: Birth of the inductively coupled-radio," Artificial Organs, vol. 26, pp. 1525-1594, 2002. 
[11] A. Sample and J. Smith, "Experimental results with two wireless power transfer systems," in Radio and Wireless Symposium, 2009. RWS '09. IEEE, Jan 2009, pp. 16-18.

[12] U. Madawala and D. Thrimawithana, "A bidirectional inductive power interface for electric vehicles in v2g systems," Industrial Electronics, IEEE Transactions on, vol. 58, no. 10, pp. 4789-4796, Oct 2011.

[13] W. Zhang, S.-C. Wong, C. Tse, and Q. Chen, "Analysis and comparison of secondary series- and parallel-compensated inductive power transfer systems operating for optimal efficiency and load-independent voltagetransfer ratio," Power Electronics, IEEE Transactions on, vol. 29, no. 6, pp. 2979-2990, June 2014.

[14] S. Cheon, Y.-H. Kim, S.-Y. Kang, M. L. Lee, J.-M. Lee, and T. Zyung, "Circuit-model-based analysis of a wireless energy-transfer system via coupled magnetic resonances," Industrial Electronics, IEEE Transactions on, vol. 58, no. 7, pp. 2906-2914, July 2011.

[15] A. Kurs, A. Karalis, R. Moffatt, J. D. Joannopoulos, P. Fisher, and M. Soljacic, "Wireless power transfer via strongly coupled magnetic resonances," Science, vol. 317, no. 5834, pp. 83-86, Jul 2007. [Online]. Available: http://dx.doi.org/10.1126/science.1143254

[16] S. Ho, J. Wang, W. Fu, and M. Sun, "A comparative study between novel witricity and traditional inductive magnetic coupling in wireless charging," Magnetics, IEEE Transactions on, vol. 47, no. 5, pp. 1522-1525, May 2011.

[17] R. M. Andre Kurs and M. Soljaxic, "Simultaneous mid-range power transfer to multiple devices," Applied Physics Letters, vol. 96, no. 4, 2010. [Online]. Available: http://scitation.aip.org/content/aip/journal/apl/ 96/4/10.1063/1.3284651

[18] I.-J. Yoon and H. Ling, "Investigation of near-field wireless power transfer in the presence of lossy dielectric materials," Antennas and Propagation, IEEE Transactions on, vol. 61, no. 1, pp. 482-488, Jan 2013.

[19] H. Haus and W. Huang, "Coupled-mode theory," Proceedings of the IEEE, vol. 79, no. 10, pp. 1505-1518, Oct 1991.

[20] A. Karalis, J. Joannopoulos, and M. Soljacic, "Efficient wireless non-radiative mid-range energy transfer," Annals of Physics, vol. 323, no. 1, pp. $34-48,2008$, january Special Issue 2008. [Online]. Available: http://www.sciencedirect.com/science/article/pii/S0003491607000619

[21] M. Kiani and M. Ghovanloo, "The circuit theory behind coupled-mode magnetic resonance-based wireless power transmission," Circuits and Systems I: Regular Papers, IEEE Transactions on, vol. 59, no. 9, pp. 20652074, Sept 2012. 
[22] C. Kong, "A general maximum power transfer theorem," Education, IEEE Transactions on, vol. 38, no. 3, pp. 296-298, Aug 1995.

[23] C.-Y. Huang, J. Boys, G. Covic, and S. Ren, "Lcl pick-up circulating current controller for inductive power transfer systems," in Energy Conversion Congress and Exposition (ECCE), 2010 IEEE, 2010, pp. 640-646.

[24] Z. Pantic, S. Bai, and S. Lukic, "Zcs lcc-compensated resonant inverter for inductive-power-transfer application," Industrial Electronics, IEEE Transactions on, vol. 58, no. 8, pp. 3500-3510, 2011.

[25] A. Sample, D. Meyer, and J. Smith, "Analysis, experimental results, and range adaptation of magnetically coupled resonators for wireless power transfer," Industrial Electronics, IEEE Transactions on, vol. 58, no. 2, pp. 544-554, Feb 2011.

[26] Y.-H. Kim, S.-Y. Kang, M.-L. Lee, B.-G. Yu, and T. Zyung, "Optimization of wireless power transmission through resonant coupling," in Compatibility and Power Electronics, 2009. CPE '09., May 2009, pp. 426-431.

[27] J. Park, Y. Tak, Y. Kim, Y. Kim, and S. Nam, "Investigation of adaptive matching methods for near-field wireless power transfer," Antennas and Propagation, IEEE Transactions on, vol. 59, no. 5, pp. 1769-1773, May 2011.

[28] T. P. Duong and J.-W. Lee, "Experimental results of high-efficiency resonant coupling wireless power transfer using a variable coupling method," Microwave and Wireless Components Letters, IEEE, vol. 21, no. 8, pp. 442-444, Aug 2011.

[29] C.-J. Chen, T.-H. Chu, C.-L. Lin, and Z.-C. Jou, "A study of loosely coupled coils for wireless power transfer," Circuits and Systems II: Express Briefs, IEEE Transactions on, vol. 57, no. 7, pp. 536-540, July 2010.

[30] H. Hoang, S. Lee, Y. Kim, Y. Choi, and F. Bien, "An adaptive technique to improve wireless power transfer for consumer electronics," in Consumer Electronics (ICCE), 2012 IEEE International Conference on, Jan 2012, pp. 359-360.

[31] S. D. Barman, A. W. Reza, N. Kumar, M. E. Karim, and A. B. Munir, "Wireless powering by magnetic resonant coupling: Recent trends in wireless power transfer system and its applications," Renewable and Sustainable Energy Reviews, vol. 51, pp. 1525 - 1552, 2015. [Online]. Available: http://www.sciencedirect.com/science/article/pii/ S1364032115006784

[32] Qi Specification, Version 1.1.2, Jun. 2013 [Online]. Available: http://www.wirelesspowerconsortium.com/developers/specification.html Std. 
[33] R. Tseng, B. von Novak, S. Shevde, and K. Grajski, "Introduction to the alliance for wireless power loosely-coupled wireless power transfer system specification version 1.0," in Wireless Power Transfer (WPT), 2013 IEEE, May 2013, pp. 79-83.

[34] Texas Instruments, Integrated Wireless Power Supply Receiver, Qi, bq51013A datasheet, Sept. 2012 [Revised Oct.2013].

[35] S. ZhuoChao, "Integrated design of the switching mode dc-dc converters." $\mathrm{Ph} . \mathrm{D}$. dissertation, Electrical \& Electronic Engineering, nanyang Technological University, 2014.

[36] B. Choi, Pulsewidth Modulated DC-to-DC Power Conversion: Circuits, Dynamics, and Control Designs. John Wiley \& Sons, 2013.

[37] B. Sahu and G. Rincon-Mora, "An accurate, low-voltage, cmos switching power supply with adaptive on-time pulse-frequency modulation (pfm) control," Circuits and Systems I: Regular Papers, IEEE Transactions on, vol. 54, no. 2, pp. 312-321, Feb 2007.

[38] S. Kapat, S. Banerjee, and A. Patra, "Modeling and analysis of dc-dc converters under pulse skipping modulation," in TENCON 2008 - 2008 IEEE Region 10 Conference, Nov 2008, pp. 1-6.

[39] W.-R. Liou, T.-H. Chen, Y.-L. Kuo, T.-Y. Huang, and M.-L. Yeh, "A high efficiency dual-mode buck converter ic for portable applications," in Communications, Circuits and Systems, 2007. ICCCAS 2007. International Conference on, July 2007, pp. 1011-1015.

[40] K.-G. Moh, F. Neri, S. Moon, P. Yeon, J. Yu, Y. Cheon, Y. seong Roh, M. Ko, and B.-H. Park, "A fully integrated $6 \mathrm{w}$ wireless power receiver operating at $6.78 \mathrm{mhz}$ with magnetic resonance coupling," in Solid-State Circuits Conference - (ISSCC), 2015 IEEE International, Feb 2015, pp. $1-3$.

[41] H.-G. Park, J.-H. Jang, H.-J. Kim, Y.-J. Park, S. Oh, Y. Pu, K. C. Hwang, Y. Yang, and K.-Y. Lee, "A design of a wireless power receiving unit with a high-efficiency 6.78-mhz active rectifier using shared dlls for magneticresonant a4 wp applications," Power Electronics, IEEE Transactions on, vol. 31, no. 6, pp. 4484-4498, June 2016.

[42] H.-M. Lee and M. Ghovanloo, "An adaptive reconfigurable active voltage doubler/rectifier for extended-range inductive power transmission," in Solid-State Circuits Conference Digest of Technical Papers (ISSCC), 2012 IEEE International, Feb 2012, pp. 286-288.

[43] J.-H. Choi, S.-K. Yeo, S. Park, J.-S. Lee, and G.-H. Cho, "Resonant regulating rectifiers (3r) operating for $6.78 \mathrm{mhz}$ resonant wireless power transfer (rwpt)," Solid-State Circuits, IEEE Journal of, vol. 48, no. 12, pp. 2989-3001, Dec 2013. 
[44] X. Li, C.-Y. Tsui, and W.-H. Ki, "A $13.56 \mathrm{mhz}$ wireless power transfer system with reconfigurable resonant regulating rectifier and wireless power control for implantable medical devices," Solid-State Circuits, IEEE Journal of, vol. 50, no. 4, pp. 978-989, April 2015.

[45] Z. Pantic and S. Lukic, "Framework and topology for active tuning of parallel compensated receivers in power transfer systems," Power Electronics, IEEE Transactions on, vol. 27, no. 11, pp. 4503-4513, 2012.

[46] Y.-H. Lam, W.-H. Ki, and C.-Y. Tsui, "Integrated Low-Loss CMOS Active Rectifier for Wirelessly Powered Devices," Circuits and Systems II: Express Briefs, IEEE Transactions on, vol. 53, no. 12, pp. 1378-1382, Dec 2006.

[47] H.-K. Cha, W.-T. Park, and M. Je, "A CMOS Rectifier With a CrossCoupled Latched Comparator for Wireless Power Transfer in Biomedical Applications," Circuits and Systems II: Express Briefs, IEEE Transactions on, vol. 59, no. 7, pp. 409-413, July 2012.

[48] S. Guo and H. Lee, "An efficiency-enhanced cmos rectifier with unbalanced-biased comparators for transcutaneous-powered high-current implants," Solid-State Circuits, IEEE Journal of, vol. 44, no. 6, pp. 17961804, June 2009.

[49] Y.-H. Lam, W.-H. Ki, and C.-Y. Tsui, "Integrated low-loss cmos active rectifier for wirelessly powered devices," Circuits and Systems II: Express Briefs, IEEE Transactions on, vol. 53, no. 12, pp. 1378-1382, Dec 2006.

[50] N. Fletcher and T. Rossing, The Physics of Musical Instruments. Berlin,Germany: Springer-Verlag, 1998.

[51] Y. Zhang and Z. Zhao, "Frequency splitting analysis of two-coil resonant wireless power transfer," IEEE Antennas and Wireless Propagation Letters, vol. 13, pp. 400-402, 2014.

[52] R. Huang, B. Zhang, D. Qiu, and Y. Zhang, "Frequency splitting phenomena of magnetic resonant coupling wireless power transfer," IEEE Transactions on Magnetics, vol. 50, no. 11, pp. 1-4, Nov 2014.

[53] P. Baxandall, "Transistor sine-wave lc oscillators. some general considerations and new developments," Proceedings of the IEE - Part B: Electronic and Communication Engineering, vol. 106, no. 16, pp. 748-758, May 1959.

[54] N.-J. Park, D.-Y. Lee, and D.-S. Hyun, "A power-control scheme with constant switching frequency in class-d inverter for induction-heating jar application," Industrial Electronics, IEEE Transactions on, vol. 54, no. 3, pp. 1252-1260, June 2007.

[55] H. Sarnago, O. Lucia, A. Mediano, and J. Burdio, "Analytical model of the half-bridge series resonant inverter for improved power conversion efficiency and performance," Power Electronics, IEEE Transactions on, vol. 30, no. 8, pp. 4128-4143, Aug 2015. 
[56] X. Wei, H. Sekiya, T. Nagashima, M. Kazimierczuk, and T. Suetsugu, "Steady-state analysis and design of class-d zvs inverter at any duty ratio," Power Electronics, IEEE Transactions on, vol. 31, no. 1, pp. 394-405, Jan 2016.

[57] J. Casanova, Z. N. Low, and J. Lin, "Design and optimization of a classe amplifier for a loosely coupled planar wireless power system," Circuits and Systems II: Express Briefs, IEEE Transactions on, vol. 56, no. 11, pp. 830-834, Nov 2009.

[58] T. Nagashima, K. Inoue, X. Wei, E. Bou, E. Alarcon, M. Kazimierczuk, and H. Sekiya, "Analytical design procedure for resonant inductively coupled wireless power transfer system with class-e 2 dc-dc converter," in Circuits and Systems (ISCAS), 2014 IEEE International Symposium on, June 2014, pp. 113-116.

[59] X. Wei, T. Nagashima, M. Kazimierczuk, H. Sekiya, and T. Suetsugu, "Analysis and design of class EM power amplifier," Circuits and Systems I: Regular Papers, IEEE Transactions on, vol. 61, no. 4, pp. 976-986, April 2014.

[60] Y. Zhang, R. Bondade, D. Ma, and S. Abedinpour, "An integrated sido boost power converter with adaptive freewheel switching technique," in Energy Conversion Congress and Exposition (ECCE), 2010 IEEE, Sept 2010, pp. 3516-3522.

[61] C. M. Chen, T. W. Liao, K. H. Hsu, and C. C. Hung, "A single-inductor multiple-output boost converter with freewheel charge-pump control," in ESSCIRC (ESSCIRC), 2012 Proceedings of the, Sept 2012, pp. 157-160.

[62] D. Ma and W. H. Ki, "Fast-transient pccm switching converter with freewheel switching control," IEEE Transactions on Circuits and Systems II: Express Briefs, vol. 54, no. 9, pp. 825-829, Sept 2007.

[63] D. Ma, W.-H. Ki, and C.-Y. Tsui, "A pseudo-cem/dcm simo switching converter with freewheel switching," IEEE Journal of Solid-State Circuits, vol. 38, no. 6, pp. 1007-1014, June 2003.

[64] K. T. Kwan and W. H. Ki, "Freewheel duration adjustment circuits for charge-control single-inductor dual-output switching converters," in Circuits and Systems (ISCAS), Proceedings of 2010 IEEE International Symposium on, May 2010, pp. 2722-2725.

[65] A. F. Bakan, N. Altintas, and I. Aksoy, "An improved psfb pwm dc-dc converter for high-power and frequency applications," IEEE Transactions on Power Electronics, vol. 28, no. 1, pp. 64-74, Jan 2013.

[66] K. Jin, M. Xu, and F. C. Lee, "A switching-capacitor pwm dc-dc converter and its variations," IEEE Transactions on Power Electronics, vol. 25, no. 1, pp. 24-32, Jan 2010. 
[67] P. Das and G. Moschopoulos, "A comparative study of zero-currenttransition pwm converters," IEEE Transactions on Industrial Electronics, vol. 54, no. 3, pp. 1319-1328, June 2007.

[68] I. O. Lee and G. W. Moon, "Phase-shifted pwm converter with a wide zvs range and reduced circulating current," IEEE Transactions on Power Electronics, vol. 28, no. 2, pp. 908-919, Feb 2013.

[69] W. Yu, J. S. Lai, W. H. Lai, and H. Wan, "Hybrid resonant and pwm converter with high efficiency and full soft-switching range," IEEE Transactions on Power Electronics, vol. 27, no. 12, pp. 4925-4933, Dec 2012.

[70] J. M. Miller and A. Daga, "Elements of wireless power transfer essential to high power charging of heavy duty vehicles," IEEE Transactions on Transportation Electrification, vol. 1, no. 1, pp. 26-39, June 2015.

[71] S. Samanta and A. K. Rathore, "A new current-fed clc transmitter and lc receiver topology for inductive wireless power transfer application: Analysis, design, and experimental results," IEEE Transactions on Transportation Electrification, vol. 1, no. 4, pp. 357-368, Dec 2015.

[72] — "Analysis and design of current-fed (1)(c) (lc) converter for inductive wireless power transfer (iwpt)," in Energy Conversion Congress and Exposition (ECCE), 2015 IEEE, Sept 2015, pp. 5724-5731.

[73] C. T. Rim and G. H. Cho, "Phasor transformation and its application to the $\mathrm{dc} / \mathrm{ac}$ analyses of frequency phase-controlled series resonant converters (src)," IEEE Transactions on Power Electronics, vol. 5, no. 2, pp. 201211, Apr 1990.

[74] C. T. Rim, "Unified general phasor transformation for ac converters," IEEE Transactions on Power Electronics, vol. 26, no. 9, pp. 2465-2475, Sept 2011.

[75] J. Zerad, S. Riachy, P. Toussaint, and J. P. Barbot, "Novel phasor transformation for feedback control design of induction heating systems with experimental results," IEEE Transactions on Industrial Electronics, vol. 62, no. 10 , pp. 6478-6485, Oct 2015.

[76] G. B. Joung, C. T. Rim, and G. H. Cho, "An integral cycle mode control of series resonant converter," in Power Electronics Specialists Conference, 1988. PESC '88 Record., 19th Annual IEEE, April 1988, pp. 575-582 vol.2.

[77] W. P. R. Ned Mohan, Tore M. Undeland, Power Electronics: Converters, Applications, and Design. Wiley; 3 edition, September 2002.

[78] K. Kotani, A. Sasaki, and T. Ito, "High-efficiency differential-drive cmos rectifier for uhf rfids," IEEE Journal of Solid-State Circuits, vol. 44, no. 11, pp. 3011-3018, Nov 2009. 
[79] P. T. Theilmann, C. D. Presti, D. J. Kelly, and P. M. Asbeck, "A uw complementary bridge rectifier with near zero turn-on voltage in sos cmos for wireless power supplies," IEEE Transactions on Circuits and Systems I: Regular Papers, vol. 59, no. 9, pp. 2111-2124, Sept 2012.

[80] S. S. Hashemi, M. Sawan, and Y. Savaria, "A high-efficiency low-voltage cmos rectifier for harvesting energy in implantable devices," IEEE Transactions on Biomedical Circuits and Systems, vol. 6, no. 4, pp. 326-335, Aug 2012.

[81] Y. Zhang, T. Lu, Z. Zhao, K. Chen, F. He, and L. Yuan, "Wireless power transfer to multiple loads over various distances using relay resonators," IEEE Microwave and Wireless Components Letters, vol. 25, no. 5, pp. 337-339, May 2015.

[82] Y. Zhu, F. Zhuo, F. Wang, B. Liu, and Y. Zhao, "A wireless load sharing strategy for islanded microgrid based on feeder current sensing," IEEE Transactions on Power Electronics, vol. 30, no. 12, pp. 6706-6719, Dec 2015.

[83] D. Ahn and S. Hong, "Wireless power transfer resonance coupling amplification by load-modulation switching controller," IEEE Transactions on Industrial Electronics, vol. 62, no. 2, pp. 898-909, Feb 2015.

[84] D. Maurath, P. F. Becker, D. Spreemann, and Y. Manoli, "Efficient energy harvesting with electromagnetic energy transducers using active lowvoltage rectification and maximum power point tracking," IEEE Journal of Solid-State Circuits, vol. 47, no. 6, pp. 1369-1380, June 2012.

[85] C. Wang, Z. Li, K. Zhao, and Q. Guo, "Efficient self-powered convertor with digitally controlled oscillator-based adaptive maximum power point tracking and rf kick-start for ultralow-voltage thermoelectric energy harvesting," IET Circuits, Devices Systems, vol. 10, no. 2, pp. 147-155, 2016.

[86] J. A. D. Lima and W. A. Pimenta, "A gm-c ramp generator for voltage feedforward control of dc-dc switching regulators," in Circuits and Systems, 2007. ISCAS 2007. IEEE International Symposium on, May 2007, pp. 1919-1922.

[87] G. D. Srivastava and R. D. Kulkarni, "Design amp; development of microcontroller based programmable ramp generator for ac-dc converter for simulating decay power transient in experimental facility for nuclear power plants," in Nascent Technologies in the Engineering Field (ICNTE), 2015 International Conference on, Jan 2015, pp. 1-6.

[88] V. Michal, "Modulated-ramp pwm generator for linear control of the boost converters power stage," IEEE Transactions on Power Electronics, vol. 27, no. 6, pp. 2958-2965, June 2012.

[89] Y. Cho, H. Miwa, and J. S. Lai, "A digital single-loop control of multiphase dc-dc converter for fuel cell powered truck auxiliary power unit," in 
Power Electronics and ECCE Asia (ICPE ECCE), 2011 IEEE 8th International Conference on, May 2011, pp. 2261-2266.

[90] G. A. Forghani-zadeh, H. Pooya; Rincn-Mora, "Low-power cmos ramp generator circuit for dc-dc converters," Journal of Low Power Electronics, vol. 2, no. 3, pp. 437-441, December 2013.

[91] A. Berger, M. Agostinelli, S. Vesti, J. A. Oliver, J. A. Cobos, and M. Huemer, "A wireless charging system applying phase-shift and amplitude control to maximize efficiency and extractable power," IEEE Transactions on Power Electronics, vol. 30, no. 11, pp. 6338-6348, Nov 2015.

[92] P. S. Riehl, A. Satyamoorthy, H. Akram, Y. C. Yen, J. C. Yang, B. Juan, C. M. Lee, F. C. Lin, V. Muratov, W. Plumb, and P. F. Tustin, "Wireless power systems for mobile devices supporting inductive and resonant operating modes," IEEE Transactions on Microwave Theory and Techniques, vol. 63, no. 3, pp. 780-790, March 2015.

[93] d. ahn, S. Kim, S. W. Kim, J. Moon, and I. K. Cho, "Wireless power transmitter and receiver supporting 200-khz and 6.78-mhz dual-band operation without magnetic field canceling," IEEE Transactions on Power Electronics, vol. PP, no. 99, pp. 1-1, 2016.

[94] K. G. Moh, F. Neri, S. Moon, P. Yeon, J. Yu, Y. Cheon, Y. s. Roh, M. Ko, and B. H. Park, "A fully integrated $6 \mathrm{w}$ wireless power receiver operating at $6.78 \mathrm{mhz}$ with magnetic resonance coupling," in Solid-State Circuits Conference - (ISSCC), 2015 IEEE International, Feb 2015, pp. 1-3.

[95] G. Lovison, M. Sato, T. Imura, and Y. Hori, "Secondary-side-only simultaneous power and efficiency control for two converters in wireless power transfer system," in Industrial Electronics Society, IECON 2015 - 41st Annual Conference of the IEEE, Nov 2015, pp. 004 824-004 829.

[96] C. Y. Wu, X. H. Qian, M. S. Cheng, Y. A. Liang, and W. M. Chen, "A $13.56 \mathrm{mhz} 40 \mathrm{mw}$ cmos high-efficiency inductive link power supply utilizing on-chip delay-compensated voltage doubler rectifier and multiple ldos for implantable medical devices," IEEE Journal of Solid-State Circuits, vol. 49, no. 11, pp. 2397-2407, Nov 2014.

[97] E. G. Kilinc, M. A. Ghanad, F. Maloberti, and C. Dehollain, "A remotely powered implantable biomedical system with location detector," IEEE Transactions on Biomedical Circuits and Systems, vol. 9, no. 1, pp. 113123, Feb 2015.

[98] H. M. Lee and M. Ghovanloo, "A high frequency active voltage doubler in standard cmos using offset-controlled comparators for inductive power transmission," IEEE Transactions on Biomedical Circuits and Systems, vol. 7, no. 3, pp. 213-224, June 2013. 
[99] B. W. Gu, B. H. Choi, and C. T. Rim, "Resonant slip ring-less doubly-fed hyper synchronous machine with variable capacitors," in Emerging Technologies: Wireless Power (WoW), 2015 IEEE PELS Workshop on, June 2015, pp. 1-5.

[100] F. Utsunomiya, A. Tanaka, and T. Douseki, "A self-powered photosensor switch detects only rising edge of infrared-light pulse for wireless zerostandby-power wake-up receiver," in SENSORS, 2013 IEEE, Nov 2013, pp. 1-4.

[101] Z. Gu, D. Prout, and A. Chatziioannou, "An adaptive method for triggering, event validation and pulse pile-up recovery in pet," in Nuclear Science Symposium and Medical Imaging Conference (NSS/MIC), 2012 IEEE, Oct 2012, pp. 2532-2536.

[102] F. W. Y. Lau, H. H. Choi, M. A. Horowitz, and C. S. Levin, "A new ic with level-crossing adc readout architecture for pet detector signals," in Nuclear Science Symposium and Medical Imaging Conference (NSS/MIC), 2012 IEEE, Oct 2012, pp. 2486-2488.

[103] Y. H. Chen, "A high resolution fpga-based merged delay line tdc with nonlinearity calibration," in Circuits and Systems (ISCAS), 2013 IEEE International Symposium on, May 2013, pp. 2432-2435.

[104] Y. Cao, P. Leroux, W. D. Cock, and M. Steyaert, "A 0.7mw 13b temperature-stable tdc with delay-line assisted calibration," in Solid State Circuits Conference (A-SSCC), 2011 IEEE Asian, Nov 2011, pp. 361-364.

[105] J. Wu, "Several key issues on implementing delay line based tdcs using fpgas," IEEE Transactions on Nuclear Science, vol. 57, no. 3, pp. 15431548, June 2010.

[106] Y. Shim, Y. Jo, S. Kim, S. Kim, and K. Cho, "A register controlled delay locked loop using a tdc and a new fine delay line scheme," in Circuits and Systems, 2006. ISCAS 2006. Proceedings. 2006 IEEE International Symposium on, May 2006, pp. 4 pp.-.

[107] C. Sauer, M. Stanacevic, G. Cauwenberghs, and N. Thakor, "Power harvesting and telemetry in cmos for implanted devices," IEEE Transactions on Circuits and Systems I: Regular Papers, vol. 52, no. 12, pp. 2605-2613, Dec 2005.

[108] T. Lehmann and Y. Moghe, "On-chip active power rectifiers for biomedical applications," in 2005 IEEE International Symposium on Circuits and Systems, May 2005, pp. 732-735 Vol. 1.

[109] Y. Zhang, Z. Zhao, and K. Chen, "Frequency splitting analysis of magnetically-coupled resonant wireless power transfer," in Energy Conversion Congress and Exposition (ECCE), 2013 IEEE, Sept 2013, pp. 2227-2232. 
[110] — - "Frequency-splitting analysis of four-coil resonant wireless power transfer," IEEE Transactions on Industry Applications, vol. 50, no. 4, pp. 2436-2445, July 2014.

[111] E. Bou, R. Sedwick, and E. Alarcon, "Maximizing efficiency through impedance matching from a circuit-centric model of non-radiative resonant wireless power transfer," in Circuits and Systems (ISCAS), 2013 IEEE International Symposium on, May 2013, pp. 29-32.

[112] I. Nam, R. Dougal, and E. Santi, "General optimal design method for series-series resonant tank in loosely-coupled wireless power transfer applications," in Applied Power Electronics Conference and Exposition (APEC), 2014 Twenty-Ninth Annual IEEE, March 2014, pp. 857-866.

[113] W. Zhang, S.-C. Wong, C. Tse, and Q. Chen, "Design for efficiency optimization and voltage controllability of series series compensated inductive power transfer systems," Power Electronics, IEEE Transactions on, vol. 29, no. 1, pp. 191-200, Jan 2014.

[114] X. Luo, S. Niu, S. L. Ho, and W. N. Fu, "A design method of magnetically resonanting wireless power delivery systems for bio-implantable devices," IEEE Transactions on Magnetics, vol. 47, no. 10, pp. 3833-3836, Oct 2011.

[115] M. Pinuela, D. C. Yates, S. Lucyszyn, and P. D. Mitcheson, "Maximizing dc-to-load efficiency for inductive power transfer," IEEE Transactions on Power Electronics, vol. 28, no. 5, pp. 2437-2447, May 2013.

[116] L. Chen, S. Liu, Y. C. Zhou, and T. J. Cui, "An optimizable circuit structure for high-efficiency wireless power transfer," IEEE Transactions on Industrial Electronics, vol. 60, no. 1, pp. 339-349, Jan 2013.

[117] M. Kiani and M. Ghovanloo, "A figure-of-merit for designing highperformance inductive power transmission links," IEEE Transactions on Industrial Electronics, vol. 60, no. 11, pp. 5292-5305, Nov 2013.

[118] M. E. Halpern and D. C. Ng, "Optimal tuning of inductive wireless power links: Limits of performance," IEEE Transactions on Circuits and Systems I: Regular Papers, vol. 62, no. 3, pp. 725-732, March 2015.

[119] X. Zhang, S. L. Ho, and W. N. Fu, "Analysis and optimization of magnetically coupled resonators for wireless power transfer," IEEE Transactions on Magnetics, vol. 48, no. 11, pp. 4511-4514, Nov 2012.

[120] W. Zhang, J. C. White, A. M. Abraham, and C. C. Mi, "Loosely coupled transformer structure and interoperability study for ev wireless charging systems," IEEE Transactions on Power Electronics, vol. 30, no. 11, pp. 6356-6367, Nov 2015.

[121] T. D. Yeo, D. Kwon, S. T. Khang, and J. W. Yu, "Design of maximum efficiency tracking control scheme for closed loop wireless power charging system employing series resonant tank," IEEE Transactions on Power Electronics, vol. PP, no. 99, pp. 1-1, 2016. 
[122] M. Iordache, D. Niculae, L. I. Bobaru, and L. Mandache, "Circuit analysis of frequency splitting phenomena in wireless power transfer systems," in Advanced Topics in Electrical Engineering (ATEE), 2015 9th International Symposium on, May 2015, pp. 146-151.

[123] Y. L. Lyu, F. Y. Meng, G. H. Yang, B. J. Che, Q. Wu, L. Sun, D. Erni, and J. L. W. Li, "A method of using nonidentical resonant coils for frequency splitting elimination in wireless power transfer," IEEE Transactions on Power Electronics, vol. 30, no. 11, pp. 6097-6107, Nov 2015.

[124] P. Si, A. P. Hu, J. W. Hsu, M. Chiang, Y. Wang, S. Malpas, and D. Budgett, "Wireless power supply for implantable biomedical device based on primary input voltage regulation," in Industrial Electronics and Applications, 2007. ICIEA 2007. 2nd IEEE Conference on, May 2007, pp. 235-239.

[125] L. Cheng, W. H. Ki, T. T. Wong, T. S. Yim, and C. Y. Tsui, "A 6.78mhz $6 \mathrm{w}$ wireless power receiver with a 3 -level $1 \mathrm{x} / 2 \mathrm{x} / 0 \mathrm{x}$ reconfigurable resonant regulating rectifier," in 2016 IEEE International Solid-State Circuits Conference (ISSCC), Jan 2016, pp. 376-377.

[126] J. u. w. Hsu, A. P. Hu, and A. Swain, "Fuzzy logic-based directional fullrange tuning control of wireless power pickups," IET Power Electronics, vol. 5, no. 6, pp. 773-781, July 2012.

[127] X. Li, X. Meng, C. Y. Tsui, and W. H. Ki, "Reconfigurable resonant regulating rectifier with primary equalization for extended coupling- and loading-range in bio-implant wireless power transfer," IEEE Transactions on Biomedical Circuits and Systems, vol. 9, no. 6, pp. 875-884, Dec 2015.

[128] M. S. Chinthavali, O. C. Onar, J. M. Miller, and L. Tang, "Single-phase active boost rectifier with power factor correction for wireless power transfer applications," in Energy Conversion Congress and Exposition (ECCE), 2013 IEEE, Sept 2013, pp. 3258-3265.

[129] N. Y. Kim, K. Y. Kim, Y. H. Ryu, J. Choi, D. Z. Kim, C. Yoon, Y. K. Park, and S. Kwon, "Automated adaptive frequency tracking system for efficient mid-range wireless power transfer via magnetic resonanc coupling," in Microwave Conference (EuMC), 2012 42nd European, Oct 2012, pp. 221-224.

[130] M. Fu, H. Yin, X. Zhu, and C. Ma, "Analysis and tracking of optimal load in wireless power transfer systems," IEEE Transactions on Power Electronics, vol. 30, no. 7, pp. 3952-3963, July 2015.

[131] W. X. Zhong and S. Y. R. Hui, "Maximum energy efficiency tracking for wireless power transfer systems," IEEE Transactions on Power Electronics, vol. 30, no. 7, pp. 4025-4034, July 2015.

[132] L. Yuan, B. Li, Y. Zhang, F. He, K. Chen, and Z. Zhao, "Maximum efficiency point tracking of the wireless power transfer system for the battery charging in electric vehicles," in Electrical Machines and Systems 
(ICEMS), 2015 18th International Conference on, Oct 2015, pp. 11011107.

[133] K. Na, H. Jang, H. Ma, and F. Bien, "Tracking optimal efficiency of magnetic resonance wireless power transfer system for biomedical capsule endoscopy," IEEE Transactions on Microwave Theory and Techniques, vol. 63, no. 1, pp. 295-304, Jan 2015.

[134] H. Li, J. Li, K. Wang, W. Chen, and X. Yang, "A maximum efficiency point tracking control scheme for wireless power transfer systems using magnetic resonant coupling," IEEE Transactions on Power Electronics, vol. 30, no. 7, pp. 3998-4008, July 2015.

[135] K. W. E. Cheng, "Computation of the ac resistance of multistranded conductor inductors with multilayers for high frequency switching converters," IEEE Transactions on Magnetics, vol. 36, no. 4, pp. 831-834, Jul 2000 .

[136] A. Reatti and M. K. Kazimierczuk, "Comparison of various methods for calculating the ac resistance of inductors," IEEE Transactions on Magnetics, vol. 38, no. 3, pp. 1512-1518, May 2002.

[137] I. C. H. Lai and M. Fujishima, "A new on-chip substrate-coupled inductor model implemented with scalable expressions," IEEE Journal of SolidState Circuits, vol. 41, no. 11, pp. 2491-2499, Nov 2006.

[138] M. Liu, M. Fu, and C. Ma, "Parameter design for a 6.78-mhz wireless power transfer system based on analytical derivation of class e currentdriven rectifier," IEEE Transactions on Power Electronics, vol. 31, no. 6, pp. 4280-4291, June 2016.

[139] Q. Deng, J. Liu, D. Czarkowski, M. K. Kazimierczuk, M. Bojarski, H. Zhou, and W. Hu, "Frequency-dependent resistance of litz-wire square solenoid coils and quality factor optimization for wireless power transfer," IEEE Transactions on Industrial Electronics, vol. 63, no. 5, pp. 28252837, May 2016.

[140] S. S. Mohan, M. del Mar Hershenson, S. P. Boyd, and T. H. Lee, "Simple accurate expressions for planar spiral inductances," IEEE Journal of SolidState Circuits, vol. 34, no. 10, pp. 1419-1424, Oct 1999.

[141] D. G. Nottiani and F. Leccese, "A simple method for calculating lumped parameters of planar spiral coil for wireless energy transfer," in Environment and Electrical Engineering (EEEIC), 2012 11th International Conference on, May 2012, pp. 869-872.

[142] H. Jiang, S. Zhou, D. Lan, J. Zhang, H. Shahnasser, K. Goldman, and S. Roy, "A parallel-trace high-q planar spiral coil for biomedical implants," in Microwave Symposium Digest (MTT), 2012 IEEE MTT-S International, June 2012, pp. 1-3. 
[143] K. Kawabe, H. Koyama, and K. Shirae, "Planar inductor," IEEE Transactions on Magnetics, vol. 20, no. 5, pp. 1804-1806, Sep 1984.

[144] S. I. Babic and C. Akyel, "New analytic-numerical solutions for the mutual inductance of two coaxial circular coils with rectangular cross section in air,' IEEE Transactions on Magnetics, vol. 42, no. 6, pp. 1661-1669, June 2006.

[145] L. R. Nerone, "Design of a 2.5-mhz, soft-switching, class-d converter for electrodeless lighting," IEEE Transactions on Power Electronics, vol. 12, no. 3, pp. 507-516, May 1997.

[146] Fairchild Semiconductor, Phase Modulated PWM Topology with the ML4818, Application Note 42026, June. 1996.

[147] M. A. de Rooij, "The zvs voltage-mode class-d amplifier, an eganfetenabled topology for highly resonant wireless energy transfer," in Applied Power Electronics Conference and Exposition (APEC), 2015 IEEE, March 2015, pp. 1608-1613.

[148] M. d. Rooij, "Performance comparison for a4wp class-3 wireless power compliance between egan fet and mosfet in a zvs class d amplifier," in PCIM Europe 2015; International Exhibition and Conference for Power Electronics, Intelligent Motion, Renewable Energy and Energy Management; Proceedings of, May 2015, pp. 1-8.

[149] D. Czarkowski and M. K. Kazmierczuk, "Zvs class d series resonant inverter-discrete-time state-space simulation and experimental results," IEEE Transactions on Circuits and Systems I: Fundamental Theory and Applications, vol. 45, no. 11, pp. 1141-1147, Nov 1998.

[150] C. Takami, T. Mishima, and M. Nakaoka, "A new zvs phase shiftcontrolled class d full-bridge high-frequency resonant inverter for induction heating," in Electrical Machines and Systems (ICEMS), 2012 15th International Conference on, Oct 2012, pp. 1-6.

[151] X. Wei, H. Sekiya, T. Nagashima, M. K. Kazimierczuk, and T. Suetsugu, "Steady-state analysis and design of class-d zvs inverter at any duty ratio," IEEE Transactions on Power Electronics, vol. 31, no. 1, pp. 394-405, Jan 2016.

[152] H. Sekiya, N. Sagawa, and M. K. Kazimierczuk, "Analysis of class de amplifier with nonlinear shunt capacitances at any grading coefficient for high q and 25no. 4, pp. 924-932, April 2010.

[153] P. Si, A. Hu, S. Malpas, and D. Budgett, "A frequency control method for regulating wireless power to implantable devices," Biomedical Circuits and Systems, IEEE Transactions on, vol. 2, no. 1, pp. 22-29, March 2008. 